\title{
PAPERS and FLASH SESSIONS
}




\section{MR-guided Focused Ultrasound Thalamotomy for Essential Tremor - one year results}

W. Jeff Elias, MD, University Of Virginia, Charlottesville, VA; Diane Huss, DPT, Charlottesville, VA; Tiffini Voss, MD, Charlottesville, VA; Johanna Loomba, BS, Charlottesville, VA; Mohamad Khaled, MD, Charlottesville, VA; Eyal Zadicario, Msc, Insightec, Inc., Israel; Robert Frysinger, Phd, Charlottesville, VA; Scott Sperling, Psyd, Charlottesville, VA; Scott Wylie, Phd, Nashville, TN; Jason Druzgal, MD, Phd, Charlottesville, VA; Binit Shah, MD, Charlottesville, VA; Madaline Harrison, MD, Charlottesville, VA; Max Wintermark, MD, Charlottesville, VA

Introduction: Recent advances in transducer technology have now enabled for the delivery of focused ultrasound (FUS) so that precise transcranial lesioning is now possible. MRI allows for contemporary stereotactic targeting and real-time monitoring of the treatment location and intensity with MR thermometry.

Methods: An FDA-approved, phase 1 clinical trial was conducted in 15 patients with medication-refractory essential tremor where a unilateral Vim thalamotomy was performed with MRgFUS. Safety was monitored with adverse event recording and serial MRI. Initial efficacy was determined with the clinical rating scale for tremor (CRST), the quality of life questionaire for ET, and the physical performance test of daily activities.

Results: FUS lesioning was accurate and tolerated without anesthesia. Side effects were generally mild, transient, and consisted of residual sensory sequelae in four patients. Upper limb tremor improved by $75 \%$ on the CRST at one year without significant change in the ipsilateral, untreated hand. Total CRST scores improved by 56\%. Quality of life and functional abilities were also significantly improved. Neuromodulation was observed in four patients such that targeting could be adjusted during the procedure.

Conclusions: Transcranial MRgFUS is feasible with initial safety and efficacy similar to other surgical procedures for tremor. A multicenter, pivotal study is being designed to further investigate this nonsurgical procedure.
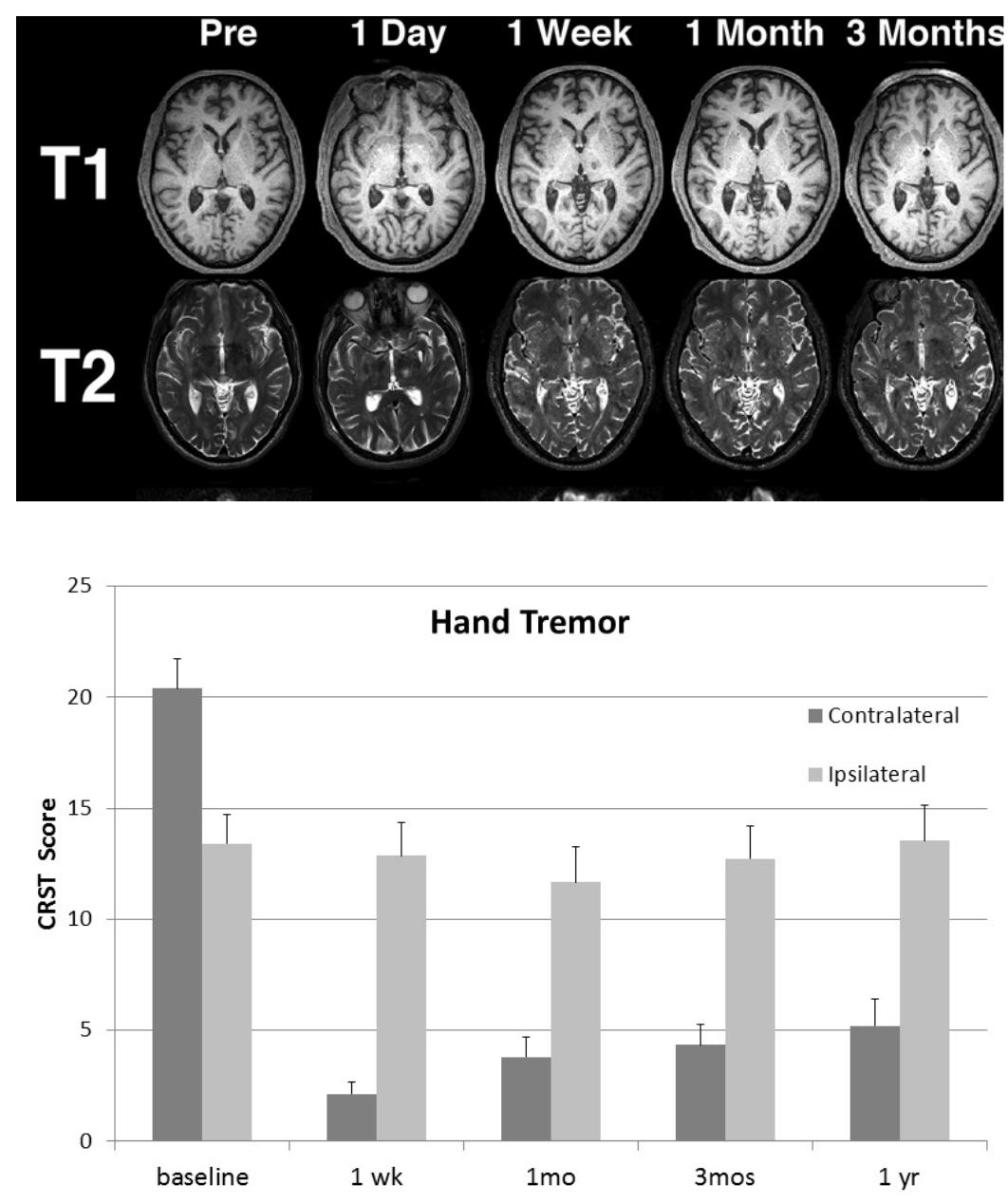

Stereotact Funct Neurosurg 2013;91(suppl 1): 1-334 - Page 2 


\section{Stereotactic Implantation of Cells Secreting Nerve Growth Factor (NGF) in The Basal Forebrain of Alzheimer Patients: Update With Improved Cell Line}

Bengt Linderoth, Karolinska Institutet, Stockholm, Sweden; Per Almqvist, MD PhD, Dept Clin Neurosci Karolinska Institutet Sthlm, Sweden; Göran Lind, MD PhD, Dept Clin Neurosci Karolinska Institutet, Sweden; Helga Eyjolfsdottir, MD, Karolinska Institutet Stockholm, Sweden; Åke Seiger, MD PhD, Karolinska Institutet Stockholm, Sweden; Maria Eriksdotter, MD PhD, Karolinska Institutet Stockholm, Sweden; Lars U. Wahlberg, MD PhD, NsGene Inc, Providence, RI

Introduction: Degeneration of cholinergic neurons in the basal forebrain correlates with cognitive decline in patients with Alzheimer's disease (AD). These neurons depend on nerve growth factor (NGF) for survival. We have developed a technique where implanted encapsulated cells release NGF to cholinergic neuron populations. The aims of this study were to demonstrate that this technique is safe and well-tolerated - now with a higher dosage.

Methods: The catheter-like cell implant consists of an NGF producing, genetically engineered human cell line encapsulated within a semipermeable hollow fiber membrane that allows for the influx of nutrients and the efflux of NGF. (Fig.1). A total of 10 patients with mild to moderate AD have been implanted bilaterally. Patients were monitored regarding safety, tolerability, cognitive functions and with a variety of biomarkers.

Results: All patients were implanted safely and accurately. The first 3 patients received bilateral implants in the nucleus basalis of Meynert (Ch4), and the following 7 were implanted both in $\mathrm{Ch} 4$ and in the vertical limb of the diagonal band (Ch2)(Fig. 2).. The first six patients were studied for 12 months. Two of these patients showed improved cognitive scores, and increased cortical $11 \mathrm{C}$-nicotine binding., Due to low levels of NGF released from retrieved devices at 12 months, a second generation implants were developed. Four AD patients were implanted with these devices and studied for 6 months. The higher dose was well tolerated. Device performance was markedly improved regarding cell survival and NGF production post explantation. Some clinical data correlate well with device performance.

Conclusions: A new cell-based treatment of AD and other neurodegenerative diseases by local delivery of NGF to targets in the brain was tested in an open-label Phase $1 \mathrm{~b}$ clinical study. Safety, tolerability and device functionality will be presented including data on a New generation device.

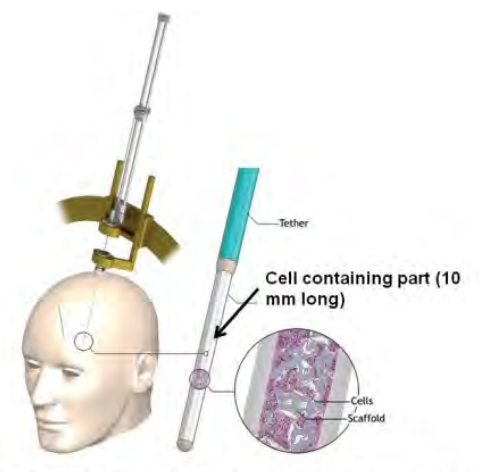

Fig. 1. Schematic illustration of implantation device. (Adapted from Wahlberg LU et al. J Neurosurg. 117(2):340-7, 2012.)

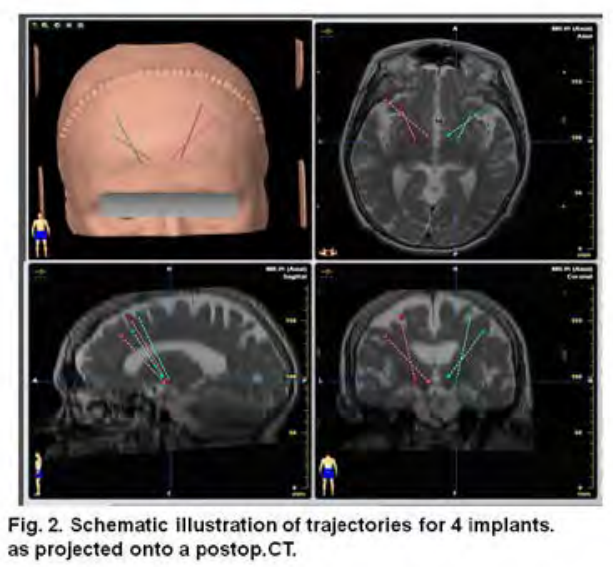

Stereotact Funct Neurosurg 2013;91(suppl 1): 1-334 - Page 3 


\section{Presentation Number: 410}

\section{Optical Imaging of Inter-areal Connectivity Evoked by Electrical Stimulation in Somatosensory and Motor Cortices}

Katherine Kudyba, BS, Vanderbilt University School of Medicine, Nashville, TN; Robert Friedman, PhD, Nashville, TN; Anna Roe, $\mathrm{PhD}$, Nashville, TN

Introduction: Cortical electrical stimulation (ES) has been shown to influence behavior and modulate perception; still, the manner in which ES acts on the underlying neuronal network to account for these effects is not well understood. The present study seeks to better understand the neuronal circuitry activated by ES in primary somatosensory and motor cortices.

Methods: Data were obtained from anesthetized Squirrel Monkeys ( $\mathrm{N}=2$ ). Following functional mapping with optical imaging of digit representation in response of vibratory tactile stimuli of finger pads, a microelectrode was placed in the superficial and deep cortical layers of an imaged digit domain ( $350 \mu \mathrm{m}$ and 1200-1400 $\mu \mathrm{m}$ below the surface respectively). Trains of electrical stimulation (bipolar, $0.4 \mathrm{~ms}$ pulse width, $250 \mathrm{~Hz}, 250 \mathrm{~ms}, 15-300 \mu \mathrm{A}$ ) were applied and optical images were acquired. Receptive field mapping of the imaged domains were also collected in response to tactile stimulation.

Results: We find increasing electrical stimulus intensity leads to a greater area of local activation, as measured with optical imaging $(\mathrm{p}<0.001)$, and that at certain thresholds additional areas of activation are observed. ES of area $3 \mathrm{~b}$ showed both local activation and additional activation sites in areas 1, 2, 3a, and M1. In superficial layers current amplitudes greater than $15 \mu \mathrm{A}$ and in deep layers amplitudes greater than $50 \mu \mathrm{A}$ elicited these secondary areas of activation. ES of area 3a showed activation locally and in adjacent modules in area 3a and M1. These secondary areas of activation appeared with ES at $100 \mu \mathrm{A}$ in superficial layers, but not deep layers. Conclusions: To summarize, cortical ES evoked inter-areal activation patterns that appear to be consistent with known connectivity observed in anatomical tracing studies. Optical imaging of ES is an exciting approach to mapping cortical circuitry. Future studies will examine plasticity in these stimulated neuronal networks.

Supported by NIH R01NS-44375.
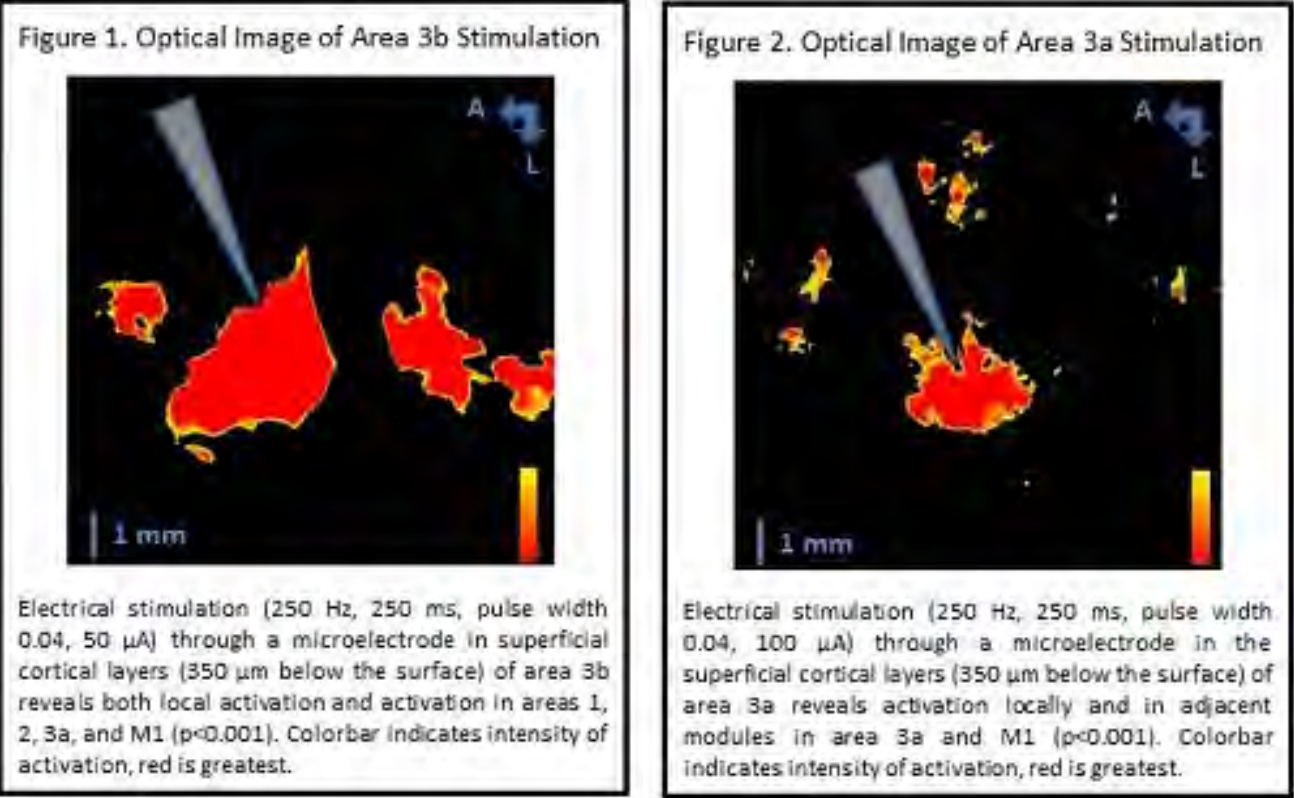
Presentation Number: 204

\section{Implanted Brain Computer Interface as a Neurofeedback System for Neurorehabilitation} Vincent ROUALDES, MD, University Hospital Nantes, Nantes, France

Introduction: There is increasing evidence of Mental Motor Imaging practice efficiency for functionnal rehabilitation after brain lesions. This is of particular interest in upper limb and hand deficits that standard physical practice fails to improve. This subjective ability is difficult to evaluate, and maybe even to achieve for patients. Brain Computer Interface (BCI) technology can guide mental practice online by analysing brain activity and translating it into a direct comprehensible feedback. Mental imaging frees brain activity from pathological afferent/efferent loops and should increase brain plasticity.

Methods: Two chronic hemiparetic patients (post-traumatic and post-stroke) where implanted whith epidural electrodes for Motor Cortex Stimulation as a neuropathic pain treatment. During the test period, a BCI system was used to evaluate from electrocorticographic signal the possibility to control and improve Mental Imaging and the subsequent effect on motor performance. Results: Both were able to control the BCI system after a few trials thanks to the great quality of the electrocorticographic signal offering significantly highter levels of information than EEG. Furthermore both achieved to use this feedback to increase their own brain activity. This immediatly resulted in an increase of motor performance including strengh, amplitude and velocity of movement. Conclusions: Minimal Invasive BCI using implanted epidural electrodes could constitute a powerfull way to teach paretic brain lesionned patients, how to produce a normal hight intensity cortical activity as a way to increase brain plasticity. Increasing evidence support this hypothesis and should soon lead to a new kind of neurorehabilitation protocols. 
Session Title: Papers Session - Emerging Technologies II

Session Time: Tuesday, May 28, 2013, 10:50 am - 11:50 am

Presentation Number: 19

Progress In Bionic Vision Devices.

Jeffrey V. Rosenfeld, MD, MS, FRACS,FRCS(Edin), FACS, Monash University, Alfred Hospital, Melbourne, VIC, Australia; Philip Lewis, B Appl Sci, Alfred Hospital, Melbourne, VIC, Australia

Introduction: There is a long history of electrical stimulation of the CNS for induction of visual percepts (phosphenes). Bionic vision devices are developing rapidly in the preclinical phase and a retinal device (Second Sight) is commercially available for blind individuals. The main indication so far is retinitis pigmentosa. Retinal, optic nerve, lateral geniculate body and visual cortex are all possible stimulation sites.

Methods: The various stimulation sites will be reviewed. The indications for retinal versus cortical devices will be presented as well as the theoretical advantages and disadvantages of each. The engineering challenges and the number and type of electrodes for functional implants will be discussed.

Results: A brief history of bionic vision will be presented. The pioneering results of the cortical stimulation devices of Doctors Brindley and Dobelle will be presented. The results of the retinal device implants (Second Sight and the Melbourne Vision Group) in blind human subjects will be presented. The components and progress of the multi-tiled multi-electrode cortical visual prosthetic device of Monash University will be presented. This is a collaborative project between the Departments of Electrical and Computer Systems Engineering, Physiology, Surgery and Ophthalmology at Monash University and the Alfred Hospital. Commercial partners are MiniFAB ${ }^{\mathrm{TM}}$ and Grey Innovation. The miniaturization of the electronics, advanced wireless technology and the advanced computer vision processing algorithms based on robotic vision take this technology to an exciting stage of practical human application. The first human implant is planned for early 2014.

Conclusions: Retinal prosthetics are improving rapidly but many blind individuals will be unsuitable candidates. The development of a cortical implant for bionic vision is achievable. Our University/private industry commercial partnership is a strong model for translational research in neurosurgery. The standardised biophysical assessment of the recipients will be vital. 


\section{Presentation Number: 333}

\section{Stereotactic Laser Ablation of Epileptic Foci: A Novel, Minimally Invasive Technique for Intractable Epilepsy}

Jon T. Willie, MD, PhD, Atlanta, GA; Ashok Gowda, PhD, Houston, TX; Amit Saindane, MD, Atlanta, GA; Sherif Nour, MD, Atlanta, GA; Robert E. Gross, MD, PhD, Emory University, Atlanta, GA

Introduction: Stereotactic laser ablation of epileptic foci -- by computer-controlled laser induced interstitial thermal therapy (LITT) with real-time magnetic resonance thermal image guidance -- is a novel minimally invasive technique to treat intractable seizures with potential advantages over traditional open resective surgery. Here we report our experience in 11 epilepsy patients: 9 with mesial temporal lobe epilepsy (MTLE), 1 with cavernous malformation (CM) and 1 with hypothalamic hamartoma (HH).

Methods: A saline-cooled fiber optic laser applicator (Visualase, Inc.) was inserted under general anesthesia utilizing a CRW stereotactic frame $(n=7)$ or Clearpoint system $(n=4)$. Laser energy was delivered during continuous MR imaging. Temperaturesensitive phase images and estimates of thermal damage during heating were superimposed on anatomical images in real-time. Standard MRI scans were obtained immediately post-procedure, with reimaging at 6 months. Prospective baseline and post-operative seizure diaries, quality of life measures, and neuropsychometric testing were performed.

Results: Nine patients (10 procedures; ages 18-64) had MTLE, 8 with $\geq 6$ month follow-up. All five patients with mesial temporal sclerosis (MTS) have had excellent or good epilepsy outcomes (Engel $1(n=4)$; Engel $2(n=1)$ ), whereas patients without MTS $(n=3)$ had poor outcomes (Engel 3-4). One patient with temporal lobe CM is seizure free at 6 months, and one patient with HH no longer experiences gelastic seizures. One serious complication, a visual field defect, occurred in one patient due to initial stereotactic rod misalignment.

Conclusions: Stereotactic laser ablation of epileptic foci is a promising, minimally invasive therapy for intractable epilepsy.

Preliminary limited results indicate that seizure outcomes in MTLE might differ in patients with and without MTS. Laser treatment of cavernous malformations and hypothalamic hamartomas is also promising. The safety and efficacy of this novel technique needs to be carefully evaluated with larger cohorts over longer periods of follow-up.
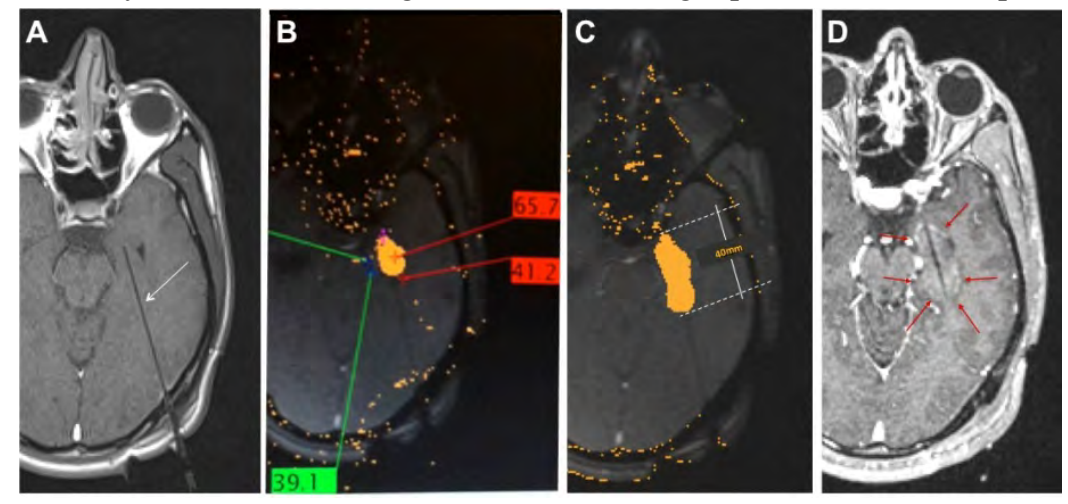


\section{Presentation Number: 351}

\section{Bone Marrow Stem Cells Intraespinal Infussion Technique As A Part Of A Pilot Study In Als}

Pedro De La Rosa Jimenez, Universitary Hospital Virgen de la Arrixaca, Murcia (Spain), Spain; Miguel Angel P'erez-Espejo Martinez, Murcia (Spain), Spain; Miguel Blanquer, Murcia (Spain), Spain; Juan Francisco Martinez-Lage Sanchez, Murcia (Spain), Spain

Introduction: ALS is a neurodegenerative disease, which treatment nowadays is unfortunately only palliative. In this context, cell therapy treatment has been proposed. As a part of a phase I study about this therapy, we developped a system to infuse the haematopoietic stem cells inside the spinal cord, at a controlled and constant speed and depth.

Methods: By using part of our functional neurosurgical armamentarium, we contructed a device that is a combination of the "microtargeting" system (Medtronic ${ }^{\circledR}$ ), with a personal prototype micro-motor that pushes the syringe at a defined speed, suspended from a Yasargyl and Leila braces. After a D3-D4 laminectomy is performed, the duramater is opened in a midline fashion, and two target points $10 \mathrm{~mm}$ apart in the veinless surface of the cord, are selected. By gentle micro-targeting manipulation, the needle is slowly introduced $6 \mathrm{~mm}$ deep into the cord, and after "on" the micromotor, $1 \mathrm{cc}$ of stem haematopoietic cells is infused in four minutes $(250 \mathrm{mcl} / \mathrm{min})$. Some gelfoam is leaved in place as puncture is completed. The procedure is repeated $10 \mathrm{~mm}$ apart. The whole operation is made under microscopical view and SSEP and MEP monitoring

Results: At the moment we have no-severe procedure-related adverse events; only some patients experienced symptoms of transient intracranial hypotension, and some experienced some small areas of hypoesthesia, paresthesia, or dysesthesia, hardly ever permanent. Conclusions: Haematopoietic stem cell therapy for ALS is nowadays already an experimental choice, even so, as a part of our study, we have developed a safe system to make the infusion at desired depth and speed, with a minimum treatment-related adverse events.
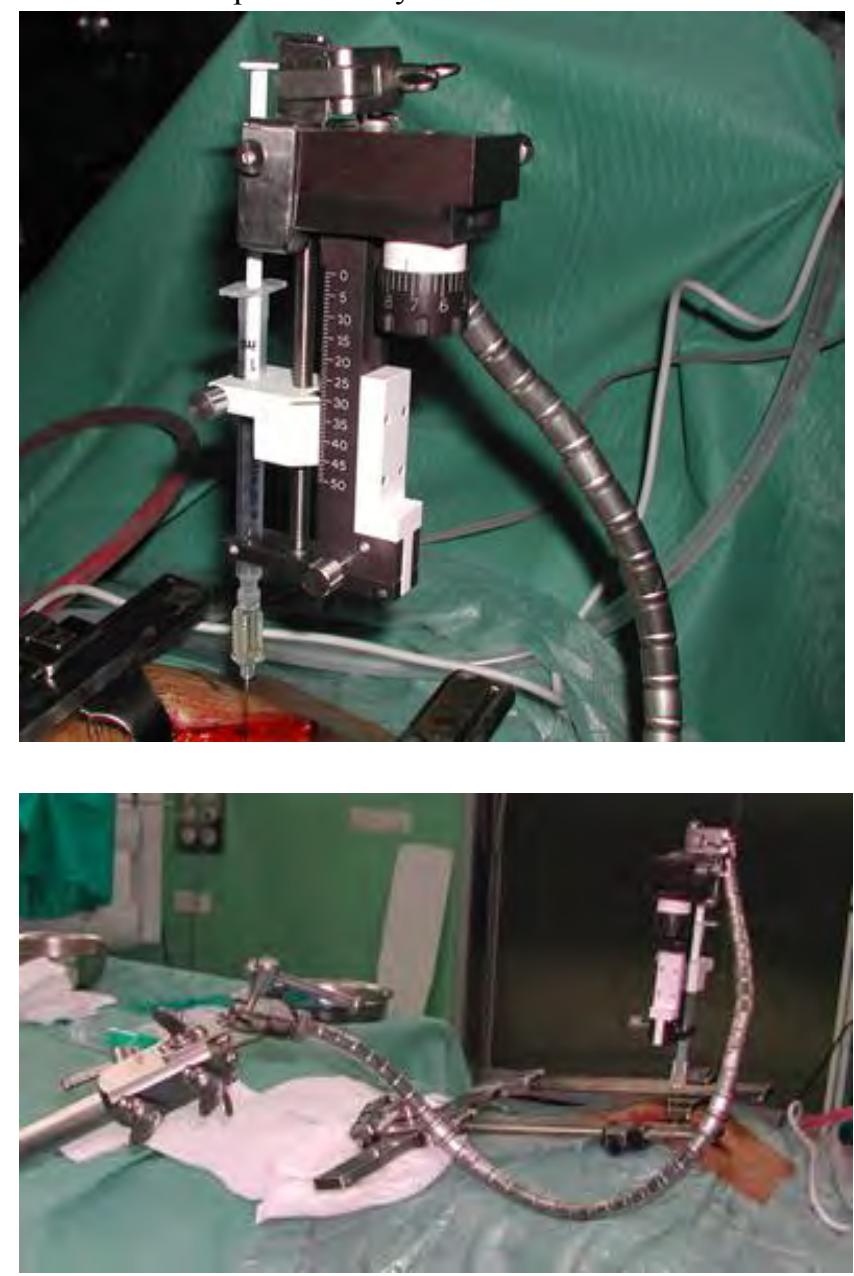
Session Title: Papers Session - Emerging Technologies II

Session Time: Tuesday, May 28, 2013, 10:50 am - 11:50 am

Presentation Number: 161

\section{New Tactics For Treatment Of Sensorineural Hearing Loss}

Jin Woo Chang, MD,PhD, Yonsei University College of Medicine, Seoul, Korea, Republic of; Jae Young Choi, MD,PhD, Seoul, Korea, Republic of

Introduction: Bilateral deafness that cannot be treated with cochlear implant is very serious problem resulted from neurofibromatosis type 2, cochlear nerve aplasia, cochlear ossification, cochlear avulsion fracture, and other congenital anomalies. From now we introduce auditory brainstem implant (ABI) as a new treatment for bilateral sensorineural hearing loss.

Methods: We have performed ABIs for 4 patients from July 2008 until now. The first case was 1 year-old boy who had congenital inner ear anomaly. The second case was 5 year-old girl who had congenital inner ear anomaly combined with mental retardation and other organ anomalies. The third case was 50 year-old woman, and she suffered from meningitis in her childhood. As she has grown up, her hearing ability decreased gradually. At her $3^{\text {rd }}$ decade, she became deaf. Her temporal bone CT showed bilateral cochlear ossification. The last case was 48 year-old man who had also cochlear ossification after inflammation. Surgery was done through retrosigmoid approach in all 4 patients. Intraoperative BAEP monitoring was done to confirm the position of electrode on cochlear nucleus of brain stem.

Results: All the procedures were done successfully without any complication except $2^{\text {nd }}$ case. In second case, we experienced displacement of electrode, so reoperation was done. The stimulation of electrode was done in about 2 months postoperatively in all the cases. The former two children had no problem during stimulation and they have needed further language therapy because they were prelingual sensorineural hearing loss. Of course we could recognize the response to sound. Latter 2 adults were postlingual hearing loss, so we could know the definite response to sound.

Conclusions: ABI could be a new tactic of surgical treatment for sensorineural hearing loss resulted from many reasons. But we think to need more experiences and further follow up to know the long term results. 
Session Title: Papers Session - Movement Disorders I

Session Time: Tuesday, May 28, 2013, 2:00 pm - 3:00 pm

\section{Presentation Number: 28}

\section{Magnetic Resonance Guided Focused Ultrasound for Unilateral Thalamotomy in Medication Refractory Essential Tremor} Nir Lipsman, University of Toronto, Toronto, ON, Canada; Michael Schwartz, MD, MSc, Toronto, ON, Canada; Yuexi Huang, PhD, Toronto, ON, Canada; Kullervo Hynynen, PhD, Toronto, ON, Canada; Tejas Sankar, MD, Edmonton, AB, Canada; Andres M. Lozano, MD, PhD, Toronto, ON, Canada

Introduction: Essential Tremor (ET) is the most common movement disorder and is associated with significant functional impairment and disability. Ablative and stimulation-based surgical treatments have been developed to treat the significant number of patients who remain symptomatic despite optimal medical management. Although often providing effective tremor control, current surgical options have risks associated with intracranial procedures, as well as issues of durability and predictability of treatment response. Here, we explore the application of MR-guided focused ultrasound (MRgFUS) as an alternative, non-invasive means of generating thalamic lesions in patients with treatment-resistant ET.

Methods: Four patients underwent awake MRgFUS thalamotomy for refractory essential tremor (mean age 70.8; mean duration of illness 17.8 years). Diagnosis of refractory ET was confirmed by a movement disorder neurologist, and patients underwent baseline medical and imaging investigations prior to treatment. Tremor assessments and serial structural imaging were performed at 1, 7, 30 and 90 days following the procedure.

Results: All four patients experienced immediate and sustained improvements in their dominant (treated) hand tremor (Figure 1). At 1- and 3-months following treatment, dominant hand tremor scores were reduced by a mean of $89 \%$ and $78 \%$, respectively, on part A of the Clinical Rating Scale for Tremor. Functional impairment decreased significantly at 3-months, with patients reporting improvements in writing, drinking and eating. There were no serious adverse events, with only one patient reporting persistent lip and finger paraesthesias at 3-months.

Conclusions: MRgFUS appears to be a safe and effective means of non-invasively generating thalamic lesions for the relief of refractory ET. This technology is a promising alternative to radiation- and surgical-based approaches to lesional functional neurosurgery, but requires larger trials with more patients to verify its efficacy.
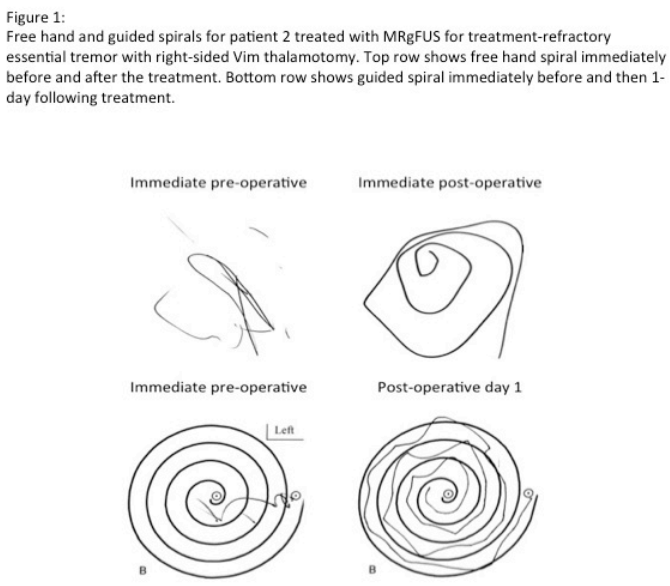


\section{High Frequency Stimulation at GPi Modulates GFRa1b, CAMK2A and GAD1 Gene Expression}

Xun Kiat Ho, B.Sc, National Neuroscience Institute, Singapore, Singapore; Yong Chee Tan, M.Sc, Singapore, Singapore; Jia Yi Tan, Dip. Sc, Singapore, Singapore; Wai Hoe Ng, MBBS, MBA, MD, FRACS, FAMS, Singapore, Singapore

Introduction: Deep brain stimulation (DBS) is an established therapy for disorders such as Parkinson's disease and dystonia. Although the efficacy of DBS is clear, its precise molecular mechanism remains unknown. Glial Cell Line Derived Factor (GDNF) family of ligands have been shown to confer neuroprotective effects on dopaminergic neurons, and putaminal infusion of GDNF have been investigated in Parkinson's Disease patients with promising results. This study aims to investigate effects of electrical stimulation on expression of GDNF family of ligands, its cognate receptors, related growth factors and calcium signaling pathways in basal ganglia.

Methods: Wistar rats were anesthetized and underwent two hours of high frequency stimulation (HFS; $2.5 \mathrm{~V}, 90 \mathrm{msec}, 130 \mathrm{~Hz}$ ) at globus pallidus interna (GPi) nucleus. A control group was subjected to similar procedure but without stimulation. Animals were sacrificed 24 and 72 hours later, and expression levels of 17 genes were measured using SYBR green real-time polymerase chain reaction (qPCR). Statistical significance of qPCR results were determined using 2-tailed independent student's t-test.

Results: HFS-treated group sacrificed 24 hours after HFS had a 3 fold decrease in mRNA expression level of GDNF family coreceptor $\alpha 1$ isoform (GFR $\alpha 1 b ; p=0.020)$, a 1.7 fold decrease in Calcium/calmodulin-dependent protein kinase II $\alpha(C A M K 2 A ; p=$ 0.005 ) and a 4.3 fold decrease in Glutamate decarboxylase 1 (GAD1; $p=0.037$ ). HFS-treated group sacrificed 72 hours after HFS had a 2 fold increase in mRNA expression level of CAMK2A $(\mathrm{p}=0.035)$.

Conclusions: Our preliminary data shows HFS at GPi modulates gene expression in basal ganglia, particularly GFR $\alpha 1 b$, CAMK2A \& GAD1. This suggests mechanism of DBS is through mediating of GDNF and calcium signaling. Further studies can elucidate this in greater depth which can lead to better design of clinical trials using these signaling pathways to halt disease progression in Parkinson's Disease and other neurodegenerative diseases.

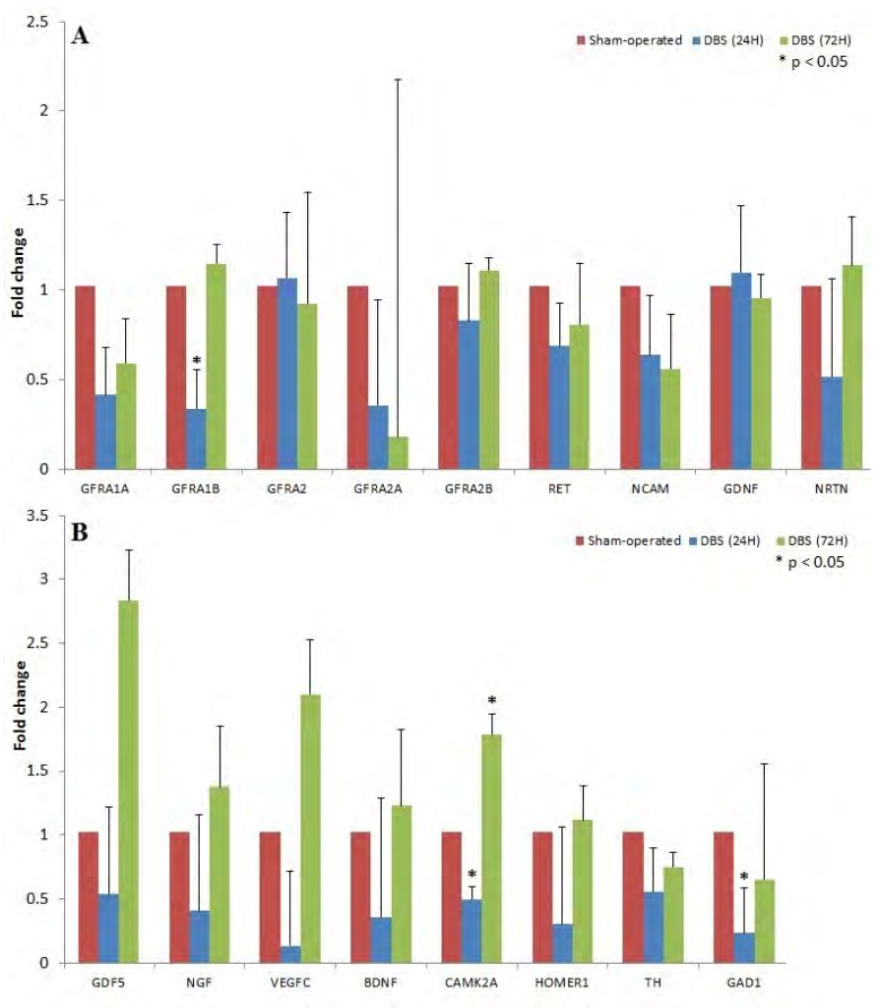


Session Title: Papers Session - Movement Disorders I

Session Time: Tuesday, May 28, 2013, 2:00 pm - 3:00 pm

Presentation Number: 312

\section{Health Economics And Surgical Treatment For Parkinson'S Disease In A World Perspective}

Gastón Schechtmann, MD PhD, Karolinska Institutet, Stockholm, Sweden; Vincent A. Jourdain, M.Sc., Laval University, QC, Canada

Introduction: Over the last two decades there has been a worldwide rebirth in the neurosurgical treatment of movement disorders. However, most studies in this field have been published by a small number of leading centers in developed countries. Consequently, knowledge about the current stereotactic surgery practices worldwide for the treatment of Parkinson's disease (PD) is biased. The objective of this study aimed to investigate the clinical practice of stereotactic neurosurgery for PD worldwide.

Methods: Neurosurgeons were contacted via email to participate in a worldwide survey. They were asked about the source of their financial support, their surgical practices for PD, complications, and the composition of their teams. The results obtained are presented in order of the countries' economical development according to the World Bank, as well as by the source of financial support.

Results: A total of 353 neurosurgeons from 51 countries who operated on 13,200 patients in 2009 were surveyed. Surgical procedures performed in high-income countries were more commonly financed by a public health-care system. In these countries, deep brain stimulation was the treatment of choice whereas lesions were kept for selected cases. In contrast, patients frequently financed surgeries themselves in lower-middle and upper-middle income countries and lesions were most commonly performed. Unexpectedly, ablative surgery is still used by about $65 \%$ of neurosurgeons, regardless of their country's economic status.

Conclusions: This study provides a previously unavailable picture of the surgical aspects of $\mathrm{PD}$ across the globe in relation to health economics and socio-demographic factors. In high-income countries national health-care programs have been successful in financially supporting neurosurgical treatments of parkinsonian patients. Global educational and training programs are warranted to raise awareness of economically viable surgical options for PD that could be adopted by public health-care systems in lower income countries. 
Session Title: Papers Session - Movement Disorders I

Session Time: Tuesday, May 28, 2013, 2:00 pm - 3:00 pm

Presentation Number: 311

\section{Double-Blind Comparison of Intraoperative Subthalamic High Frequency, Low Frequency and Sham Stimulation Effects on Verbal Fluency}

Alexander I. Tröster, PhD, Barrow Neurological Institute, Phoenix, AZ; Richard Murrow, MD, Chapel Hill, NC; Eldad Hadar, MD, Chapel Hill, NC

Introduction: Declines in verbal fluency are the most common and persistent cognitive changes after subthalamic deep brain stimulation (STN DBS) for Parkinson disease. Whereas some reported that fluency declines depend on hemispheric language dominance, others suggest that bilateral DBS is necessary. Yet others propose that the declines represent microlesion rather than stimulation effects or that fluency effects are frequency dependent. Studies addressing the potential predictive value of intraoperative stimulation have not been reported. The purpose of this study was to compare within subjects the verbal fluency effects of left and right, high and low frequency and sham STN stimulation.

Methods: 22 PD patients underwent microelectrode guided bilateral STN DBS. Location of stimulation was at the site determined to provide best motor benefit while minimizing side effects. Hemisphere stimulated first was based on body side most affected by PD. Order of frequency of stimulation (low $=50 \mathrm{~Hz}$, high $=185 \mathrm{~Hz}$ at $3.5 \mathrm{~V}$, sham $0 \mathrm{~V}$ ) was randomized and examiner and patient were blind to frequency. 6 equivalent category fluency forms were used in counterbalanced order.

Results: Repeated measures GLM disclosed poorer fluency after left than right stimulation ( $p=.05$, one-tailed). The side $x$ frequency interaction and frequency effects were not significant. Differences between left and right stimulation were observed at low ( $p=.03$, one-tailed) and high ( $\mathrm{p}=.09$, one-tailed) frequencies, but sham stimulation effects were comparable $(\mathrm{p}=.82$, two-tailed). Fluency performance did not differ between those first undergoing left or right sided surgery. Mean fluencies ranged from 10-12 words/60 sec, and SDs from 4-8 words.

Conclusions: STN stimulation effects are hemisphere dependent and not simply due to a microlesion effect. Hemispheric differences were not observed with sham stimulation. Variability in performance across trials may be too high to render intraoperative stimulation a useful fluency outcome predictor. Extraoperative studies are needed to replicate findings. 
Session Title: Papers Session - Movement Disorders I

Session Time: Tuesday, May 28, 2013, 2:00 pm - 3:00 pm

Presentation Number: 291

\section{Involvement Of Pedunculopontine (PPN) And Cuneiform Nuclei (CfN) In Bipedal Locomotor Behavior: Electrophysiological} Study In Normal And Parkinsonian (MPTP) Primate

Laurent GOETZ, INSERM U836, Grenoble, France; Brigitte PIALLAT, PhD, Grenoble, France; Manik BHATTACHARJEE, PhD, Grenoble, France; Hervé MATHIEU, PhD, Grenoble, France; Olivier DAVID, PhD, Grenoble, France; Stéphan CHABARDES, MD, Grenoble, France

Introduction: DBS of the PPN was proposed as a new strategy to treat gait disorders in Parkinson's disease. However, several studies pointed the lack of experimental data regarding the role of the PPN in the control of locomotion and in the pathophysiology of gait troubles. Using our primate model of bipedal locomotion (Goetz, 2012), we performed an electrophysiological mapping of the tegmentum at rest and during locomotion under normal and MPTP conditions.

Methods: Micro-electrode recording, EMG(quadriceps) and single unit analysis were performed on two primates under normal and MPTP condition. Recordings were performed at rest, during locomotion and transitions from wakefulness to sleep (TWS). Neurons were classified according to their discharge response to locomotion.

Results: At rest: we found an heterogeneous population of neurons within the PPN and CfN. Under MPTP, we didn't observe significant change in the mean firing rate but an increase in burst pattern. During locomotion: we could identify two main clusters of neurons: phasic neurons mainly in the anterior part of the Tegmentum time locked with EMG and tonic excitatory neurons.

Furthermore, some neurons that responded to locomotion also modified their discharge activity during TWS. Under MPTP, gait blockages could be record electrophysiologically and were reversed by apomorphine.

Conclusions: We could record for the first time in awake primate, neurons in the Tegmentum (PPN and CfN) that respond to locomotion. Interestingly, some neurons were also implicated in TWS showing the co-localisation of integration centers of locomotion and arousal in the tegmetum. Under MPTP condition, our results rule out the hypothesis of an overactive inhibition of the PPN but underline a putative role of bursting activity of the Tegmentum and dopamine depletion in the pathophysiology of gait troubles in PD. This provides important clues for the enhancement of DBS of the PPN in human. 


\section{Presentation Number: 83}

Five Hundred Stereoelectroencephalography Procedures For Presurgical Invasive Evaluation Of Drug-resistant Epilepsy: Efficiency, Safety, Application Accuracy And Outcome On Seizures.

Francesco Cardinale, MD, "Claudio Munari" Epilepsy and Parkinson Surgery Centre, Milano, Italy; Massimo Cossu, MD, Milano, Italy; Laura Castana, MD, Milano, Italy; Giuseppe Casaceli, MD, Milano, Italy; Laura Tassi, MD, Milano, Italy; Stefano Francione, MD, Milano, Italy; Lino Nobili, MD, Milano, Italy; Francesca Gozzo, MD, Milano, Italy; Paola Proserpio, MD, Milano, Italy; Giorgio Lo Russo, MD, Milano, Italy

Introduction: StereoElectroEncephaloGraphy (SEEG) is gaining popularity for the presurgical invasive evaluation of drug-resistant epilepsies.

Methods: Five hundred consecutive SEEG implantations were performed in our Center between 1996 and 2011 on 482 patients (6496 multilead intracerebral electrodes). Noninvasive anatomo-electro-clinical data of every patient were reviewed for planning a tailored strategy of exploration. All procedures were subsequently performed according to Talairach methodology, incorporating modern technologies throughout the years, such as intraoperative imaging (O-arm, Medtronic, Minneapolis, Minnesota) and robotics (Neuromate ${ }^{\circledR}$, Renishaw-mayfield SA, Nyon, Switzerland). In vivo application accuracy was measured on a sub-dataset of the 81 most recent robot-assisted procedures (1050 electrodes).

Results: The methodology allowed a successful implantation in all cases. Major complications rate was $2.6 \%$ for the earlier 419 procedures, $1.2 \%$ for the latest $81(\mathrm{p}=0.7)$. Median euclidean error was $0.78 \mathrm{~mm}$ (InterQuartile Range $0.49-1.08)$ at the entry point, $1.77 \mathrm{~mm}$ (IQ range $1.25-2.51$ ) at the target point. Concerning SEEG efficiency, 354 subjects underwent SEEG-guided tailored brain resections, 15 underwent SEEG-guided radio-frequency thermo-coagulation, 20 are waiting for surgery, 89 did not proceed to further treatment for epileptological/functional reasons and/or for patient's refusal. Three patients died while waiting for surgery for seizurerelated causes. One three year old child died the day following electrode placement due to massive brain edema and profound hyponatremia of undetermined cause. Out of 344 subjects who underwent SEEG-guided tailored brain resections with a minimum follow-up of 12 months, 194 (56.4\%) are free of disabling seizures (Engel Class I).

Conclusions: This study suggests that SEEG is safe, accurate and efficient. Traditional Talairach methodology, implemented by multimodal planning and robot-assisted surgery, allows direct electrical recording from superficial and deep-seated brain structures, providing essential information in the most complex cases of drug-resistant epilepsy.
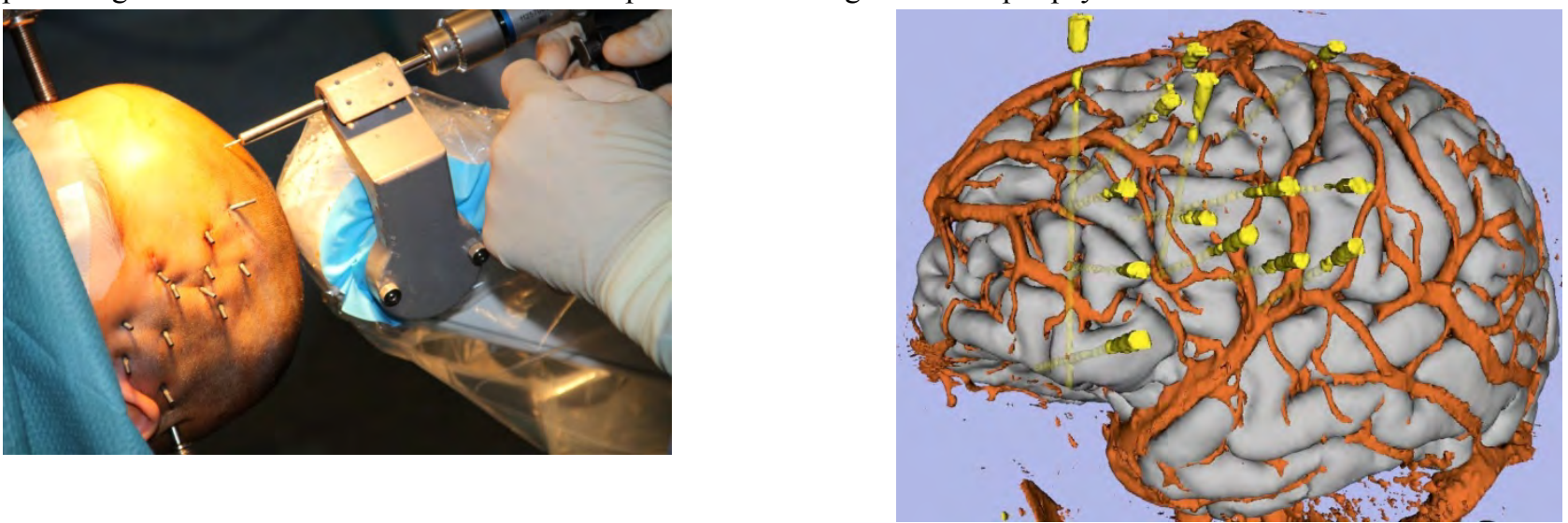


\section{Stimulation of the Anterior Nucleus of the Thalamus for Epilepsy (SANTE) Trial: Results Related to Region of Onset and Prior Surgical Treatments}

Robert E. Gross, MD, PhD, Emory University, Atlanta, GA; Robert Worth, MD, PhD, Indianapolis, IN; Thomas Witt, MD, Indianapolis, IN; Timothy Mapstone, MD, Oklahoma City, OK; Michael Kaplitt, MD, PhD, New York, NY; Ashwini Sharan, MD, Philadelphia, PA; John Neal, MD, Marshfield, WI; Andrew Shetter, MD, Phoenix, AZ; Roy Bakay, MD, Chicago, IL; Jaimie Henderson, MD, Palo Alto, CA; Gordon Baltuch, MD, Philadelphia, PA; Andrew Youkilis, MD, St. Louis, MO; Nicholas Barbaro, MD, Indianapolis, IN; Jeff Elias, MD, Charlottesville, VA; Guy McKhann, MD, New York, NY; Robert Goodman, MD, PhD, New York, NY

Introduction: The Stimulation of the Anterior Nucleus of the Thalamus for Epilepsy (SANTE) trial demonstrated significantly greater decrease in seizures at the end of the 3 month blinded phase in subjects randomized to stimulation (40.4\%) vs. shamstimulation (14.5\%) (Epilepsia, 51: 899,2010), with persistent decreases during the 9 month unblinded phase and long-term follow-up (LTFU) phase at 2 years. Here we present long-term follow-up (up to 5 years) with respect to subgroups differing in region of epilepsy onset and prior surgical treatments.

Methods: After completion of the blinded and unblinded phases of the SANTE trial (13 months), 105 subjects entered the LTFU phase allowing unrestricted adjustment of stimulation parameters and medications. Seizure outcomes in subjects remaining in the study were assessed at $1(\mathrm{~N}=99), 2(\mathrm{~N}=82), 3(\mathrm{~N}=75), 4(\mathrm{~N}=76)$ and 5 years $(\mathrm{N}=59)$. Seizure onset location was determined by the site investigator based on a clinical description of the seizures and previous diagnostic testing. Prior surgical treatments were captured within subjects' medical history. Subjects with $\geq 70$ days of seizure diary data were included.

Results: Seizure reduction at 1 year and 5 years was $41 \%$ and $69 \%$ for subjects in all categories combined; $44 \%(\mathrm{~N}=59)$ and $76 \%$ $(\mathrm{N}=33)$ for temporal lobe onsets; 53\% $(\mathrm{N}=25)$ and 59\% $(\mathrm{N}=17)$ for frontal lobe onsets; and 34\% $(\mathrm{N}=22)$ and $68 \%(\mathrm{~N}=13)$ for other lobe onsets. At 1 and 5 years, subjects with no prior surgery had seizure reduction of $45 \%(\mathrm{~N}=46)$ and $70 \%(\mathrm{~N}=31)$, whereas subjects with prior vagus nerve stimulator (VNS) had reduction of $32 \%(\mathrm{~N}=29)$ and $71 \%(\mathrm{~N}=14)$, epilepsy surgery (e.g. resection) $47 \%(\mathrm{~N}=8)$ and $65 \%(\mathrm{~N}=3)$, and those with VNS plus surgery $53 \%(\mathrm{~N}=16)$ and $69 \%(\mathrm{~N}=11)$.

Conclusions: Anterior nucleus stimulation is effective at long-term open-label follow-up in subjects with onsets from various lobes, and in subjects with prior VNS and/or epilepsy surgery. 


\section{Presentation Number: 91}

\section{Improved Seizure Outcomes in Resections Involving the Orbito-frontal Cortex: Evidence for "Orbito-frontal-plus" Epilepsy} Demitre Serletis, MD, PhD, FRCSC, Cleveland Clinic, Cleveland, OH; Jorge A. Gonzalez-Martinez, MD, PhD, Cleveland, OH; Juan Bulacio, MD, Cleveland, OH; Imad Najm, MD, Cleveland, OH; William Bingaman, MD, Cleveland, OH

Introduction: In long-term studies, surgery for frontal lobe epilepsy has poor seizure outcomes. Reasons are unclear but incomplete resection of the epileptogenic zone (EZ) is a possible cause for failures. Specifically in orbito-frontal (OF) epilepsy, we identified two pathological and electro-clinical patterns indicating different surgical approaches for each one. We present a consecutive series of patients with OF epilepsy, highlighting different surgical strategies and correlation with seizure outcome.

Methods: Eleven patients with refractory epilepsy involving the OF region were prospectively identified over three years, in whom stereoelectroencephalography (SEEG) was required to identify the EZ. All patients underwent pre-operative evaluation, SEEG and post-operative MRI. Demographic features, seizure semiology, MRI findings, EZ location, surgical resection site and pathological diagnosis were recorded and analyzed. Surgical outcome was correlated with type of resection.

Results: Post-operative seizure control improved for all patients, with no complications or mortalities. Five patients exhibited "OFplus frontal polar epilepsy", with the EZ consistently residing in the frontal lobe; following surgery, four were seizure-free (Engel I) and one improved (Engel II). The remaining six patients had multi-lobar epilepsy, with the EZ located in the OF cortex associated with the temporal polar region ("OF-plus temporal polar"). All six were seizure-free after a mean follow-up of 26.6 months. Pathology confirmed focal cortical dysplasia (FCD) I in ten patients, with FCD II identified in the remaining patient.

Conclusions: In patients with OF epilepsy, multi-lobar resections may be needed to achieve seizure freedom. Failures in frontal resections can be partially explained by incomplete resection of the EZ by leaving behind the temporal pole. 
Presentation Number: 102

Results Of Surgery For Refractory Epilepsy: Highlights From A Series Of 1696 Patients Submitted To Surgery.

Arthurt Cukiert, Clinica de Epilepsia de Sao Paulo, Sao Paulo, Brazil; Jose Burattini, Sao Paulo, Brazil; Cristine Cukiert, Sao Paulo, Brazil

Introduction: This paper reviewed a series of epileptic patients submitted to surgery in the MR era.

Methods: 1696 patients submitted to epilepsy surgery from 1996 to 2012 were studied. Mean age at surgery was 16 years and followup period was 6.6 years. 919 patients were submitted to temporal lobe, 187 to frontal, 70 to rolandic, 69 to posterior quadrant, 26 to parietal, 12 to occipital and 6 to insular cortical resection. 92 patients were submitted to hemispherectomy and 190 patients to callosotomy; 102 patients were submitted to VNS and 23 to DBS.

Results: Overall, $81 \%$ of the patients submitted to temporal lobe resection were seizure-free after surgery, as did $82 \%$ of the patients submitted to hemispherectomy. $72 \%$ of the patients with frontal lobe have been rendered seizure-free. Patients submitted to occipital, parietal and insular resections were seizure-free in $85 \%, 80 \%$ and $100 \%$ of them, respectively. Eighty-three percent of the patients submitted to posterior quadrant resections were seizure-free postoperatively. There has been an $88 \%$ reduction in the generalized seizure frequency in those patients with Lennox-Gastaut syndrome submitted to callosotomy. Overall, $51 \%$ of the patients submitted to VNS had at least a 50\% reduction in seizure frequency; best results were obtained in kids with Lennox-Gastaut syndrome. Among the DBS patients, although targeted for different syndromes, the best results were obtained with hippocampal stimulation.

Conclusions: Patients with MRI-defined lesions had better seizure-outcome. There is a clearcut trend towards operating at a younger age, and discontinuing the use electrocorticography, awake-craniotomy and Wada's testing. Neuromodulation is very likely to be increasingly used in these patients. 
Electrocorticographic Evidence Of Different Physiopathologic Types In Mesial Temporal Epilepsy. Surgical Implications. Cristina V. Torres, MD, PhD, Hospital La Princesa, Madrid, Spain; Jesús Pastor, MD, PhD, Madrid, Spain; Eduardo GarcíaNavarrete, MD, PhD, Madrid, Spain; Marta Navas-García, MD, Madrid, Spain; Ricardo Gil-Simoes, MD, Madrid, Spain; Rafael García de Sola, MD, PhD, Madrid, Spain

Introduction: Recent investigations have suggested that mesial temporal epilepsy might be divided in different subtypes, depending on the existence of a focal or a reticular origin. The aim of this study is to confirm this hypothesis and to assess the efficacy of an optimized surgical approach based on it.

Methods: Thirty patients underwent complete presurgical evaluation according to the Epilepsy Unit of Hospital La Princesa protocol, including videoelectroencephalography (VEEG) with foramen ovale electrodes, and were diagnosed with mesial temporal epilepsy and deemed candidates for resective surgery (Spencer's technique). Patients were intraoperatively evaluated by electrocorticography (ECoG) with 20 subdural electrode grids over the lateral temporal cortex, and an 8 electrode mesial strip, under low doses of sevoflurane $(0.5 \%)$ and remifentanil $(0.1 \mathrm{mg} / \mathrm{kg} / \mathrm{min})$. Fifteen minutes after the start of the ECoG, $0,1 \mathrm{mg} / \mathrm{kg}$ of iv etomidate were administered. The mesial strip was maintained until the anterior medial temporal resection was performed, and after ten minutes, a second dosage of etomidate was given. A tailored resection of the mesial structures was executed in some of the patients, according to the ECoG results.

Results: In six out of the 30 patients, the irritative interictal mesial spikes disappeared following the anterior medial temporal resection, and mesial structures were spared (Figure 1A). All of the patients are in Engel's class I at the last follow-up (mean 23 months after surgery). In the remaining patients, the irritative mesial activity increased or was unchanged (Figure 1B), and the amygdale and the hippocampus were excised. At the last follow-up (mean 16 months after surgery), these patients are in Engel's class I: $83 \%$, II: $8 \%$, III: $8 \%$.

Conclusions: Our results strongly suggest the existence of physiopathologic differences within the mesial temporal epilepsy syndrome. The identification of these different subtypes is fundamental for an individualized surgical approach and for the consideration of new therapeutic strategies.

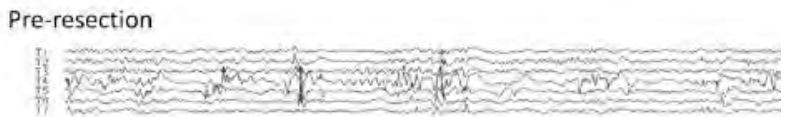

A Post-resection
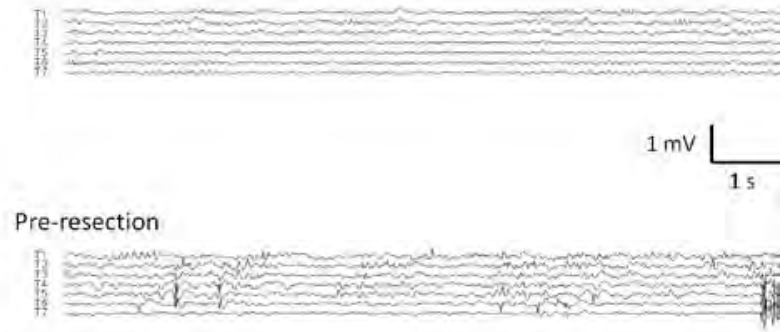

i Post-resection

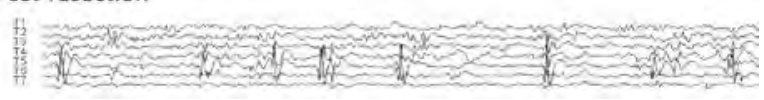




\section{Presentation Number: 164}

\section{Clinician Preferences in Management of Trigeminal Neuralgia - A survey of Australian Neurosurgeons and Neurologists} Benjamin P. Jonker, MB BS MMed(Clin Epi) FRACS, University of Sydney, Camperdown, Australia; Mark Dexter, MB BS FRACS, Camperdown, Australia; Zelma H.T. Kiss, MD PhD, Calgary, AB, Canada; Michael Solomon, MB BCh MSc FRACS, Camperdown, Australia; Jane Young, MBBS MPH PhD FAFPHM, Camperdown, Australia

Introduction: We surveyed a large cohort of Australian neurosurgeons and neurologists concerning which trigeminal neuralgia (TN) patients are suitable for surgery versus pharmacological treatment. We also examined whether consensus exists concerning which patients are suitable for microvascular decompression (MVD) versus percutaneous procedures versus radiosurgery. Finally, we asked in which scenarios they might participate in clinical research.

Methods: Following ethics committee approval a survey was administered to all Australian neurosurgeons and to a sample of Australian neurologists. Nine combinations of TN symptom severity and medication side effects were presented and respondents indicated whether they preferred surgery versus ongoing medical management on a bidirectional linear scale. Neurosurgeons were asked which procedure type was preferred across a range of clinical scenarios. Respondents were asked whether they would participate in various clinical studies.
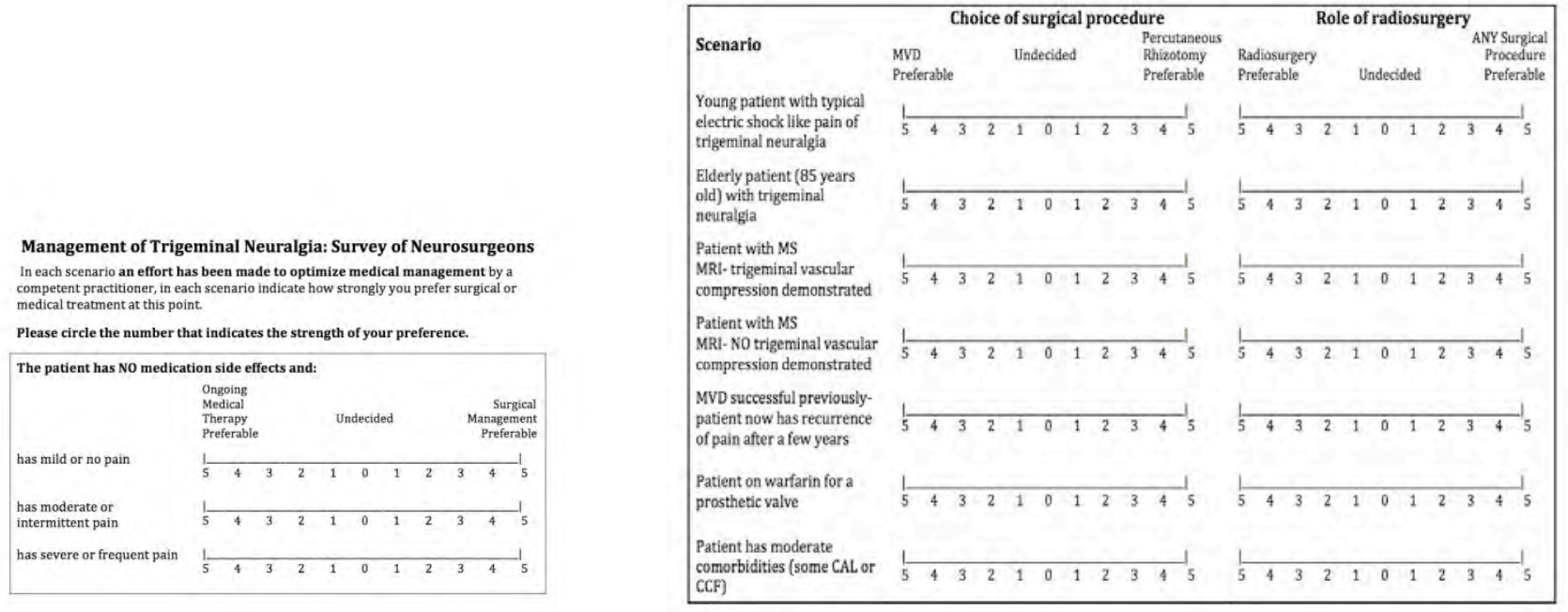

Results: 226 neurosurgeons and 146 neurologists were invited to participate with a response rate of $52.7 \%$. For the majority (6/9) of TN scenarios strong preferences exist concerning whether surgery or ongoing medical management is preferable. There was no difference between neurosurgeons and neurologists concerning which treatment was preferred in each scenario. For young patients with typical pain MVD was preferred over alternatives. In more complicated TN scenarios (recurrence post MVD, elderly patients, medical comorbidities) there was equipoise concerning choice of MVD versus percutaneous rhizotomy. In these cases radiosurgery was usually considered a reasonable option. Neurologists and neurosurgeons expressed a willingness to participate in studies including randomized trials although only a minority of neurosurgeons would participate in a trial comparing MVD to radiosurgery.

Conclusions: Neurologists and neurosurgeons exhibited similar preferences for surgery versus medical management. Whilst in principle a majority would be prepared to participate in clinical trials there are only a limited number of scenarios for which adequate equipoise exists, and the small numbers of patients might make RCTs unfeasible. 
Session Title: Papers Session - Radiosurgery

Session Time: Tuesday, May 28, 2013, 2:00 pm - 3:00 pm

Presentation Number: 35

\section{A New Grading System Focusing On Functional Preservation For Brain Metastasis Patients Treated With Stereotactic Radiosurgery}

Toru Serizawa, MD, PhD, Tsukiji Neurological Clinic, Tokyo, Japan; Yoshinori Higuchi, MD, PhD, Chiba University, Chiba, Japan; Osamu Nagano, MD, PhD, Chiba Cardiovascular Center, Ichihara, Japan; Shinji Matsuda, MD, PhD, Chiba Cardiovascular Center, Ichihara, Japan; Junichi Ono, MD, PhD, Chiba Cardiovascular Center, Ichihara, Japan; Naokatsu Saeki, MD, PhD, Chiba University, Chiba, Japan

Introduction: Functional preservation (qualitative survival, QS) has been regarded as one of the ideal endpoints for evaluating treatment results for patients with brain metastases. However, previous reports on grading systems for brain metastasis patients, i.e. original RPA, SIR, BSBM, GPA and modified RPA, are based on only overall survival (OS), not QS. Herein, we propose a new grading system for predicting both OS and QS in patients undergoing gamma knife radiosurgery (GKS).

Methods: Among 2481 brain metastasis cases, treated with GKS, we verified common poor prognostic factors for OS and QS.

Results: There were 1490 men and 991 women. Median age was 65, range 7-94. The most common primary site was the lung (1629 cases), followed by gastro-intestine (315), breast (268), uro-genital (147) and others (122). Median survival times (MSTs) were 7.7 months for OS and 46.4 months for QS. The common significant prognostic factors were initial KPS score (poor factor; $<70$, $\mathrm{p}<0.0001$ ), maximum tumor diameter $(\geq 25 \mathrm{~mm}, \mathrm{p}<0.0001)$, brain lesion number (multiple, $\mathrm{p}=0.0002$ ), and extra-cranial disease status (active, $\mathrm{p}=0.0044$ ). According to the sum of these 4 risk factors, we classify the "low" risk group (547 cases) as having a score of 0 or 1; "intermediate" a score of 2 (1176); and "high" (758) a score of 3 or 4. MSTs for QS were 71.9 months in the "low", 42.3 in the "intermediate" and 19.0 in the "high" risk group. There were highly significant differences among the adjacent groups ( $<<0.0001$, 0.0002). MSTs for OS were 15.5 months in the "low", 7.8 in the "intermediate" and 4.9 in the "high" risk group. Differences among groups were also significant $(\mathrm{p}<0.0001)$.

Conclusion: Our new index, the QS score (QSS), was found to be uniquely useful for predicting both OS and QS simultaneously in brain metastasis patients undergoing GKS. 
Session Title: Papers Session - Radiosurgery

Session Time: Tuesday, May 28, 2013, 2:00 pm - 3:00 pm

Presentation Number: 352

\section{Time Delayed Contrast Enhanced MRI Improves Detection of Brain Metastases}

Marina Kushnirsky, B.A., Hofstra North Shore-LIJ School of Medicine, Manhasset, NY; Joel S. Katz, D.O., Manhasset, NY; Jonathan P.S. Knisely, M.D., Manhasset, NY; Michael Schulder, M.D., Hofstra North Shore-LIJ School of Medicine, Manhasset, NY; Lisa Rosen, ScM, Manhasset, NY; Jared Steinklein, M.D., Manhasset, NY; Craig Warshall, M.D., Manhasset, NY; Vinh Nguyen, M.D., Manhasset, NY

Introduction: Detection of brain metastases (BM) in patients undergoing stereotactic radiosurgery (SRS) may improve with a time delay following administration of intravenous contrast. Metastasis detection with time-delayed MRI was evaluated in this study. Methods: 53 volumetric MRI studies from 38 patients undergoing SRS for BM were evaluated. All studies used $0.1 \mathrm{mmol} / \mathrm{kg}$ Gadobenate dimeglumin (MultiHance ${ }^{\circledR}$ ), immediately after injection, followed by two more axial T1 sequences after 5 minute intervals. $287 \mathrm{BM}$ were identified. The studies were randomized and examined separately by 3 radiologists, who were blinded to the temporal sequence. Each recorded the number of BM detected per scan.

Results: At least one new lesion was detected in the 2nd scan as compared to the 1 st in $35.3 \%$ of subjects (95\% CI: $22.4 \%-49.9 \%$ ). The increase in BM numbers between scans 1 and 2 ranged from 1 to 10. At least 1 new lesion was detected in the 3rd scan as compared to the 2nd in $21.6 \%$ of subjects (95\% CI: 11.3\%-35.3\%). The increase in BM numbers between scans 2 and 3 ranged from 1 to 9 . Between scans 1 and 3 additional tumors were seen on $41.2 \%$ of scans (increase range 1 to 14). The median increase in tumor number for all comparisons was 1. There was a significant increase in number of BM detected from scan 1 to scan 2 ( $P<0.0367)$, and from 1 to 3 ( $\mathrm{P}<0.0264)$.

Conclusions: In patients who are being prepared for SRS of brain metastases, delayed MRI after contrast injection revealed an increased number of targets that need treatment. To avoid missing tumors that could be treated at the time of planned SRS, and resultant "treatment failures", we recommend that post-contrast MRI be acquired between 10 and 15 minutes after injection in patients undergoing SRS for treatment of metastatic brain tumors. 
Session Title: Papers Session - Radiosurgery

Session Time: Tuesday, May 28, 2013, 2:00 pm - 3:00 pm

Presentation Number: 172

Delayed Contrast Extravasation MRI diffrentiates metastatic tumor progression from radiation necrosis after Radiosurgery Roberto Spiegelmann, MD, Chaim Sheba Medical Center, Ramat Gan, Israel; Ouzi Nissim, MD, Ramat Gan, Israel; Dvora Nass, MD, Ramat Gan, Israel; Chen Hoffmann, MD, Ramat Gan, Israel; Youval Grober, MD, Ramat Gan, Israel; Lior Zach, MD, Ramat Gan, Israel; David Guez, MSc, Ramat Gan, Israel; David Last, Msc, Ramat Gan, Israel; Diane Daniels, Msc, Ramat Gan, Israel; Yael Mardor, PhD, Ramat Gan, Israel

Introduction: Radio-necrosis (RN) occurs in up to $10 \%$ of lesions treated with radiosurgery (SRS). Currently there are no reliable tools to distinguish between RN and viable tumor progression, a distinction with important clinical consequences. We assesed a novel technique based on delayed contrast extravasation MRI, for differentiating metastatic tumor progression from RN after SRS.

Methods: Thirty six patients with brain metastases were recruited. All patients were scanned by delayed contrast extravasation MRI. Enhancement subtraction maps were calculated by subtracting high resolution MR images acquired shortly after contrast injection from images acquired 75 minutes later. Patients were followed by conventional and delayed contrast extravasation MRI unless their medical condition necessitated surgical intervention. Ten patients (4 NSCLC, 4 breast cancer, one melanoma and one with adenoid cystic carcinoma) underwent surgery for 12 metastases. They were treated with a single dose of $18-20 \mathrm{~Gy}$, delivered to the $80 \%$ isodose line, $13.1 \pm 2.9$ months prior to resection.

Results: The subtraction maps revealed two distinct enhancement patterns: regions with contrast clearence from tissue at the 75 minute scan (fast) and regions where contrast accumulated with time (slow). Stereotactic biopsies from four patients (12 specimens) were taken according to the generated pre-surgical subtraction maps. The histological characteristics of each sample were compared with the corresponding regions in the maps.In all cases histological data confirmed that fast regions represent active tumor and slow regions represent non-tumoral tissue, consistent with RN. Additionally, blood vessel morphology was different in these two regions, possibly explaining the different appearance of tumor vs $\mathrm{RN}$ in the subtraction maps. Thus the subtraction maps seem to reflect vascular integrity- a surrogate marker of tissue characterization.

Conclusions: These results demonstrate the feasibility of applying delayed contrast extravasation MRI as a valuable clinical tool for distinguishing radiation induced changes from metastatic tumor progression. 
Session Title: Papers Session - Radiosurgery

Session Time: Tuesday, May 28, 2013, 2:00 pm - 3:00 pm

Presentation Number: 73

\section{Long Term Result of Gamma Knife Treatment For Prolactinomas}

Peng Li, MD, west china hospital, sichuan university, chengdu, China; Wei Wang, MD, chengdu, China

Introduction: This study is to evaluate the effects and safety of gamma knife surgery for prolactinomas by analyzing the tumor control rate, improvement of hyperprolactinemia and complications of the patients after treatment.

Methods: 101 patients with prolactinoma were followed-up after undergoing gamma knife surgery from July 2004 to November 2008 at our hospital. The prescription dose and isodose line were 11-33Gy(29.1 $\pm 2.3 \mathrm{~Gy})$ and 45-75\%. Periodical follow-up with serial MRIs and serum prolactin level check were carried out for each patient after therapy. The clinical symptoms improvement, tumor control rate and changes of endocrine were analyzed retrospectively.

Results: 86 patients finished follow-up with an average time of $51.2 \pm 5.3$ months. Obvious clinical symptoms improvement and significant tumor control were observed in 48 patients (59.3\%) and 81 patients $(94.2 \%)$. 56 patients $(65.1 \%)$ reported improvement of hyperprolactinemia. Normal serum prolactin level was observed in 37 patients. The time interval between the treatment and the hyperprolactinemia remission was $28.5 \pm 3.5$ months. Apituitarism was observed in 13 cases. No newly developed symptoms of optic nerve and other cranial nerves impairment related to the therapy were observed.

Conclusions: The gamma knife treatment may play an important role in the management for prolactinoma. Considering the slow decline of the high prolactin level, long-term follow-up is suggested. 
Session Title: Flash Session - Movement Disorders

Session Time: Tuesday, May 28, 2013, 3:30 pm - 4:30 pm

Presentation Number: 226

\section{Saccadic modulation with Concomitant Subthalamic nucleus and Pedunculopontine nucleus-stimulation in advanced} Parkinson's disease

Mohammad J. Naushahi, BMedSci, MRCS, Imperial College Neuromodulation Group, London, United Kingdom; Amad N. Khan, MBBS, MSc, London, United Kingdom; Dipankar Nandi, DPhil, FRCS (SN), London, United Kingdom

Introduction: The subthalamic nucleus (STN) is known to be involved in controlling saccades. The pedunculopontine nucleus (PPN) has extensive connections with the basal ganglia. Recently, stimulation of the PPN has been explored as a possible target to treat the axial motor symptoms (principally gait freezing and loss of postural control) of advanced Parkinson's disease (PD). This study explores the previously unreported effects of concomitant bilateral STN and PPN-stimulation on both the initiation and inhibition of saccades in advanced PD.

Methods: Five patients with advanced PD performed two different oculomotor tasks whilst OFF-stimulation, ON bilateral STNstimulation, ON bilateral PPN-stimulation and ON concomitant bilateral STN \& PPN-stimulation. The first task involved visually guided saccades (that are reflexive) and the second, antisaccades (that are volitional). Saccadic latency, accuracy and velocity were recorded for both the visually guided and antisaccade tasks, while prosaccades (errors) were measured for the antisaccade task alone. Twelve Patients with advanced PD not receiving stimulation treatment, 12 age-matched healthy subjects and 12 young healthy subjects were also investigated.

Results: Concomitant bilateral STN \& PPN-stimulation produced the greatest improvement in the mean latencies and accuracies for the visually guided saccades compared to OFF-stimulation $(\mathrm{p}<0.001)$. Interestingly, bilateral STN \& PPN-stimulation also produced a significant additional effect compared to STN-stimulation alone on the number of prosaccades $(\mathrm{p}<0.01)$ and velocity $(\mathrm{p}<0.05)$.

Conclusions: PPN-stimulation may thus influence the functional output of the superior colliculi for saccadic modulation in advanced PD in addition to the effect of STN-stimulation. This may help to explain some of the clinical effects of PPN-stimulation on axial motor function, in particular, on postural control and prevention of falls. 
Session Title: Flash Session - Movement Disorders

Session Time: Tuesday, May 28, 2013, 3:30 pm - 4:30 pm

Presentation Number: 365

\section{Anatomical Study Of The Pedunculopontine Nucleus In Humans: Where Is The Ppn}

Takashi Kawasaki, Tokyo Metropolitan Neurological Hospital, Tokyo, Japan; Masahiro Shin, MD, Tokyo, Japan; Makoto Taniguchi, MD, Tokyo, Japan; Fusako Yokochi, MD, Tokyo, Japan; Ryoichi Okiyama, MD, Tokyo, Japan; Yoshitomo Umitsu, Tokyo, Japan; Norio Ishizuka, Tokyo, Japan

Recently, the pedunculopontine nucleus (PPN) has come to be considered a possible new target for deep brain stimulation (DBS) for the treatment of Parkinson's disease. However, the description of the PPN in conventional atlases is unsatisfactory. We therefore clarified the location of the PPN using post-mortem Japanese human brains fixed with formalin and identified the target of the PPNDBS on MRI in preparation for PPN-DBS.

Three brain hemispheres from three post-mortem human brains fixed with formalin were used. Serial frozen sections of 50micrometer thickness were prepared. Kluver-Barrera (KB) staining of these sections was performed, followed by microscopic observation. Because there are many acetylcholinergic neurons in PPN, we identified the PPN by choline acetyltransferase (ChAT) staining. The center of the PPN was represented by coordinates based on the line leading from the anterior commissure to the posterior commissure (ACPC line), and PPN-DBS surgery was simulated using these coordinates on MRI with Surgiplan targeting software. An aggregation of neurons was found between the superior cerebellar peduncle and the medial lemniscus on axial KB-stained sections at the level of the boundary of the inferior midbrain and the superior pons. Furthermore, we identified ChAT-positive neurons and fibers in the same region. We therefore concluded that this region was the PPN. On average the coordinates of the center of the PPN were $7 \mathrm{~mm}$ lateral to midline, $5.6 \mathrm{~mm}$ posterior to the $\mathrm{PC}$, and $16.7 \mathrm{~mm}$ inferior to the ACPC line. These coordinates were specified in the Surgiplan software as a tentative target, and the target was illustrated on MRI of Parkinson's disease patients.

We demonstrated the location of the PPN on the frozen sections and on MRI. These data are useful for precisely locating the PPN and for planning PPN-DBS surgery. 
Session Title: Flash Session - Movement Disorders

Session Time: Tuesday, May 28, 2013, 3:30 pm - 4:30 pm

Presentation Number: 415

\section{Dorsal Versus Ventral Stn Stimulation Effects On The Inhibition Of Action Impulses}

Nelleke C. van Wouwe, PhD, Vanderbilt University Medical Center, Nashville, TN; Scott A. Wylie, PhD, Nashville, TN; Kristen Kanoff, BSc, Nashville, TN; Joseph Neimat, MD, Vanderbilt University Medical Center, Nashville, TN

Introduction: Frontal-basal ganglia circuitry dysfunction caused by Parkinson's disease (PD) impairs important executive cognitive processes, such as the ability to inhibit impulsive action tendencies. Subthalamic nucleus (STN) deep brain stimulation (DBS) in PD improves the inhibition of impulsive actions that interfere with goal-directed behavior. An unresolved question is whether this effect depends on stimulation of a particular STN subregion. We hypothesized that stimulating a relatively dorsal as opposed to ventral subregion of the STN would improve inhibition of impulsive actions.

Methods: We studied four PD patients recently implanted with STN DBS whose post operative CT, registered to the preoperative MRI and the Vanderbilt Deformable Atlas, demonstrated two adjacent contacts contained in the dorsal and ventral STN, respectively. Off medication, patients completed two testing sessions involving either low amplitude stimulation of the dorsal or ventral contact at a constant current of $0.4 \mathrm{~mA}$ (see figure 1). Patients performed a Simon reaction time task, which evokes impulsive action tendencies and measures the proficiency of inhibiting these tendencies.

Results: Relative to ventral stimulation, stimulating dorsal contacts bilaterally reduced the interference from impulsive action tendencies across all patients. The steeper reduction of interference at slower reaction times depicted in figure 2 reflects more proficient inhibition of impulsive actions with dorsal stimulation.

Conclusions: Stimulating subregions of the STN produces dissociable effects on the ability to inhibit impulsive action tendencies, with stimulation of dorsal subregions leading to improved inhibition. In the future, intraoperative and postoperative assessment of inhibitory action control may help optimize key motor and cognitive symptoms in PD. This pilot study demonstrates the importance of testing the effects of stimulating STN subregions on other executive cognitive functions linked to PD and frontal-basal ganglia circuitry. 
Session Title: Flash Session - Movement Disorders

Session Time: Tuesday, May 28, 2013, 3:30 pm - 4:30 pm

Presentation Number: 163

\section{Can Low Frequency Stimulation At Subthalamic Area Improve The Motor Performance Of Patients With Parkinson's} Disease?

Chiung Chu Chen, MD, PhD, Chang Gung Memorial Hospital, Taoyuan county, Taiwan; Wey Yil Lin, MD, Taoyuan county, Taiwan; Hsiao Lung Chan, PhD, Taoyuan county, Taiwan; Po Hsun Tu, MD, Taoyuan county, Taiwan; Shih Tseng Lee, MD, Taoyuan county, Taiwan; Chin Song Lu, MD, Taoyuan county, Taiwan; Peter Brown, MD, Oxford, United Kingdom

Introduction: Low frequency stimulation (LFS, $<=20 \mathrm{~Hz}$ ) stimulation of the subthalamic nucleus (STN) has been generally reported to worsen the measures of motor impairment in patients with Parkinson's disease (PD). The deleterious effect is more evident in patients with relatively preserved baseline performance. In those sides with worse baseline performance, however, the LFS is associated with a tendency for movement to be improved. In this study, we hypothesize that stimulation at $10 \mathrm{~Hz}$ can improve motor performance at some circumstance, given growing evidence has shown that $10 \mathrm{~Hz}$ oscillation may correlate with improved motor performance.

Methods: 15 PD patients chronically implanted with deep brain stimulation (DBS) electrodes in both STN were stimulated bilaterally at 5,10,20,50 and $130 \mathrm{~Hz}$ and the effect of the DBS on self-initiated isometric contraction made with elbow flexion (FLEX) and finger pinch (PINCH) were compared to performance without DBS.

Results: Stimulation at $10 \mathrm{~Hz}$ improved the mean peak force of FLEX by an average of $22.3 \pm 18.7 \%$ compared to no stimulation $(\mathrm{p}=0.004)$. In those sides with the best baseline performance, the relative response to $10 \mathrm{~Hz}(16.7 \pm 4.1 \%$ increase $)$ and $20 \mathrm{~Hz}$ stimulation $(10.0 \pm 3.4 \%$ decrease) show different direction $(\mathrm{p}=0.008)$.

Conclusion: the current study highlights the need to consider the baseline performance of a subject in a given task when determining the effects of low frequency STN stimulation. 
Session Title: Flash Session - Movement Disorders

Session Time: Tuesday, May 28, 2013, 3:30 pm - 4:30 pm

Presentation Number: 322

Validation Of The Electrodes' Location By Probabilistic Tractography In Parkinson's Disease Patients Who Underwent Bilateral Subthalamic Deep Brain Stimulation (DBS) Surgery

David Kis, MD, University of Szeged, Department of Neurosurgery, Szeged, Hungary; Adrienn Máté, Szeged, Hungary; Tamas

Kincses, MD, PhD, Szeged, Hungary; Loránd Erőss, MD, PhD, Budapest, Hungary; Péter Klivényi, MD, PhD, Szeged, Hungary; Pál

Barzó, MD, PhD, DsC, Szeged, Hungary

Introduction: The most common surgical target in medication refractory Parkinson's disease is the subthalamic nucleus (STN). It has 3 functional subgroups: 1, limbic; 2, associative; 3 , motor part. The surgical target is the motor part which is identified by the combination of direct visualization and stereotactical coordinates. Previously it has been proven that the connection based segmentation of the STN by probabilistic tractography can divide it to the 3 functional subgroups in healthy subjects.

Methods: The aim of our study was to test whether the connection based segmentation of the STN can be adapted to Parkinson's disease patients to verify DBS electrode positions. 9 patients who underwent bilateral STN DBS surgery were included in our study. Diffusion tensor imaging, T1, T2 and susceptibility weighted imaging (SWI) were acquired preoperatively. The STN was visualized on the T2 and SWI scans, the 7 cortical target masks (prefrontal, premotor, primary motor and sensory, dorsal parietal, temporal and occipital regions) were delineated manually on the T1 images. Postoperative CT scans were performed to localize the electrodes. Subsequently, segmentation of the STN and tractography from the effective contact were performed.

Results: After the operation adequately effective symptomatic treatment was achieved in every patient. According to the connectivity based segmentation, DBS electrodes went through the part of the STN which showed high probability connection to the premotor area. Tractography initiated from the effective contact showed connections to the cerebellum, motor thalamic nuclei, globus pallidus and the premotor cortical area.

Conclusions: According to our results, connectivity based STN segmentation by probabilistic tractography is a useful method to visualize the motor part of the STN. Therefore it could be used to verify the position of DBS electrodes, but on the other hand it also can be helpful for direct visualization in surgical planning. 
Session Title: Flash Session - Movement Disorders

Session Time: Tuesday, May 28, 2013, 3:30 pm - 4:30 pm

Presentation Number: 347

\section{Different Targets for Different Patients' Profile: how to modulate DBS for Parkinson's Disease.}

Andrea Landi, MD, San Gerardo Hospital, Monza, Italy; Andrea Trezza, MD, Monza, Italy; Angelo Antonini, MD, Venice, Italy; Alberto Vimercati, MD, Monza, Italy; Erik P. Sganzerla, MD, Monza, Italy

Introduction: The subthalamic nucleus (Stn) and the pars interna of the Globus pallidus (Gpi) are most reported targets in DBS for Parkinson's disease; Gpi is considered more effective to decrease diskinesias and Stn to reduce drugs; Gpi presents with less neuropsychological derangements than Stn; moreover, the cranial portion of Stn and the Zona Incerta are very effective for tremor. Therefore, since different patients may present with some prevailing symptoms, it seems advisable to custom the target to every different clinical features.

Methods: 275 PD patients have been studied. Targets were: Stn (229 pts), cranial Stn /Zona Incerta (22 pts), Gpi (24 pts). Inclusion criteria were: for StnDBS: classical PD symptoms, age under 65, no cognitive decline; for Gpi DBS: classical PD symptoms, age over 65, mild cognitive impairment; for cranial Stn/ZI DBS: prevalence of tremor. Patients were postoperatively evaluated with UPDRS and cognitive/neuropshychiatric tests; mean follow up is 6.2 yrs.

Results: UPDRS II and II decreased of 62\% in mean; best results were in the Stn and Stn/ZI groups versus Gpi (65\% and 67\% versus 54\%). Stn DBS obtained a decrease in LDopa eq/day of 56\%; Stn/Zona 76\%; Gpi DBS 4\%. Tremor was well controlled both in Stn and in Stn/ZI DBS. Gpi and Stn were comparable to control bradykienesia and rigidity and on-off fluctuations. Dystonic features and Pisa syndrome were better controlled with Gpi DBS. No neuropsychological nor cognitive impairment were in the Gpi group; in Stn group transient neuropsychological derangement was observed in 19\%, mainly in over 60 .

Conclusions: Most of the patients benefited from Stn DBS; moreover, in case of prevailing dystonia, in older patients and in case of slight neuropsychological or cognitive alterations, Gpi is the target of choice; PD with prevailing tremor may be treated with ZI stimulation, as an alternative to thalamic Vim DBS. 
Session Title: Flash Session - Movement Disorders

Session Time: Tuesday, May 28, 2013, 3:30 pm - 4:30 pm

Presentation Number: 295

State Changes In Subthalamic Nucleus Neurons: Implications For Motor Circuits And Deep Brain Stimulation Surgery Robert M. Brownstone, MD, PhD, FRCSC, Dalhousie University, Halifax, NS, Canada; Hans Hultborn, D.M.Sc., University of Copenhagen, Denmark

Introduction: Our understanding of the connectivity of basal ganglia (BG) neurons has led to the development and refinement of BG models to explain pathophysiological processes such as Parkinson's Disease. However, despite our understanding of connectivity, the operation of BG circuits in health and disease remains poorly understood. Studies of simple motor circuits, such as the crustacean stomatogastric ganglion (containing $<30$ neurons with well-understood connectivity), have revealed that in order to understand the function of a network, it is critical to thoroughly characterise the neuronal properties of its components. We have thus used intraoperative microstimulation to study properties of subthalamic (STN) neurons in Parkinson's Disease, and reveal that these neurons can switch between different electrophysiological states.

Methods: All recordings were performed by a single surgeon (RB) using single extracellular microelectrodes (exposed tips $\sim 40 \mu \mathrm{m}$, impedances 500-1,000 k $\Omega$ ). STN neurons were recorded in 30 patients (60 STNs) with Parkinson's Disease (between $42-73$ years, mean: 60). Neurons were stimulated through the recording electrode with parameters of $100-300 \mathrm{~Hz}$ for $<500 \mathrm{~ms}, 0.2-0.5 \mathrm{~ms}$ pulses, and $<10 \mu \mathrm{A}$.

Results: Brief trains of stimuli could lead to the cessation of firing which in some neurons followed a brief burst of action potentials. This silent period could last many seconds, and was repeatable. Microstimulation (trains or single stimuli) could also terminate silent periods leading to resumption of repetitive firing. These data are remarkably similar to "silent plateau potentials" as reported in rat STN slices (Kass and Mintz, Proc Natl Acad Sci USA, 2006, 103: 183-8).

Conclusions: We conclude that human STN neurons have different states, and that microstimulation can lead to a change in these states. We suggest that this is mediated by stimulation of afferent axons, and that this has implications for understanding and improving STN stimulation therapies. 
Session Title: Flash Session - Movement Disorders

Session Time: Tuesday, May 28, 2013, 3:30 pm - 4:30 pm

Presentation Number: 198

Striatal-transplantation Of Dopamine Neurons In Parkinson Rat Model: Subthalamic Nucleus Neuronal Activity And Gene Expression

Mesbah Alam, PhD, Hannover Medical School, Hannover, Germany; Regina Rumpel, PhD, Hannover, Germany; Alexander Klein, $\mathrm{PhD}$, Hannover, Germany; Meltem Oezer, Hannover, Germany; Maike Wesemann, Hannover, Germany; Joachim Kurt Krauss, MD,PhD, Hannover, Germany; Kerstin Schwabe, PhD, Hannover, Germany; Andreas Ratzka, PhD, Hannover, Germany; Claudia Grothe, PhD, Hannover, Germany

Introduction: In Parkinson's disease (PD), as well as in 6-hydroxy dopamine (6-OHDA)-lesioned rats dopamine (DA) depletion in the nigrostriatal system lead to basal ganglia dysfunction with neuronal hyperactivity in the subthalamic nucleus (STN), i.e., enhanced firing rate and burst activity, together with enhanced beta oscillatory activity. Intrastriatal transplantation of DA neurons has been shown to functionally re-innervate the host brain and restore DA input. To better understand the effect of striatal transplantation of DA cells on the STN in 6-OHDA lesioned rats.

Methods: In animals, which were transplanted with cells derived from the mesencephalon of E12 rat embryos in the striatum, we combined behavioral and histological findings with electrophysiological extracellular recordings, as well as qRT-PCR analyses of GABAergic and glutamatergic transporter and receptor genes.

Results: After striatal transplantation the rotational behavior induced by amphetamine injection was improved, or even overcompensated by $116 \%$ in rats with large grafts (2000-6000 cells). While burst activity was not affected, STN neuronal firing rate, as well as beta oscillatory activity was fully normalized in rats with these grafts, while small grafts were less effective. Although grafted rats displayed restored expression of the GABA synthesizing enzymes Gad65 and Gad67 in the striatum compared to naive rats, the grafts induced a decrease in NMDA receptor subunit expression. Interestingly, the NMDA receptor subunit 2B was also less expressed in the STN, both compared to 6-OHDA-lesioned and naive rats.

Conclusions: In summary, DA grafts partially restore functional deficits and neuronal activity of STN in PD rats. However, functional recovery may be compromised by changes in receptor gene expression induced by DA grafts. 


\section{The Role Of Connectivity-based Thalamic Segmentation In The Surgical Targeting In Tremor Patients}

Adrienn Máté, MD, University of Szeged, Department of Neurosurgery, Szeged, Hungary; David Kis, MD, University of Szeged, Department of Neurosurgery, Szeged, Hungary; Tamas Kincses, MD, PhD, Szeged, Hungary; Péter Klivényi, MD, PhD, Szeged, Hungary; Pál Barzó, MD, PhD, DsC, Szeged, Hungary

Introduction: In medication refractory tremor dominant extrapyramidal diseases surgical intervention can be an effective symptomatic treatment. The most common surgical targets are the ventralis intermedius (ViM) or ventralis oralis posterior (VoP) nuclei of the thalamus. Target identification is based on stereotactic maps, because thalamic nuclei cannot be identified by standard MRI modalities. It has been proven that probabilistic tractography based on diffusion tensor imaging (DTI) can be used for thalamic segmentation by its cortical connections in healthy subjects, therefore it may be useful to localize ViM and VoP in tremor patients. Methods: The aim of our study was to examine the reliability of the connectivity-based segmentation of the thalamus in tremor patients. 6 patients who underwent contralateral thalamotomy for unilateral upper extremity dominant tremor were involved in the study. We acquired DTI data preoperatively. The surgical target (ViM in 4 cases and VoP in 2 cases) was defined by the help of stereotactic maps. Postoperative MRI was performed 3 months after surgery to verify the location of the lesion. Connectivity-based thalamic segmentation was done retrospectively. ViM and VoP were identified by their connections to the primary motor (M1) and premotor (PM) cortex.

Results: There was a significant decrease in the tremor intensity in all patients during the follow-up period (six months to three years) without any disabling side effects. The lesion in ViM targeting took place at the border of the M1-PM connections and tracts from here projected to the M1, PM and cerebellum. In VoP targeting the lesion was in the middle of the PM connections and tracts from the lesion projected to the PM.

Conclusions: Connectivity-based thalamic segmentation seems to be a reliable alternative targeting method instead of stereotactic maps. Its advantage is that it adapts to individual anatomy perfectly and directly visualizes the target nuclei. 
Presentation Number: 305

Long Term Assessment Of Functional Consequences Of 150 Tibial Neurotomies For Treatment Of Spastic Foot.

Patrick MERTENS, MD, PhD, University LYON 1, LYON, France; Emile Simon, MD, LYON, France; Gustavo Polo, MD, LYON, France; Afif Afif, MD, PhD, DAMAS, Syrian Arab Republic; Marc SINDOU, MD, PhD, LYON, France

Introduction: The goal of this study is to assess with a long follow up ( $>5$ years) the functional results of selective tibial neurotomy performed to treat disabling spastic foot (equino-varus foot).

Methods: In a population of 150 consecutive operated patients, we assessed:

- Spasticity (Ashworth scale) of the targeted muscles (gastrocnemius, posterior tibialis)

- The ability to obtain the therapeutic goals defined preoperatively with the patient and the medical team

- The personal satisfactory index of each patient (0 to 10)

- The modifications of gait parameters

- The modifications of shoe wearing and splint use

- Effects on pain (0 to 10 VAS) and on antispastic drugs intake

Results: - Equinus and/or varus recurrences have been described in $8.6 \%$ of the patients. In this group of patients, $61 \%$ of them have not beneficiated of a valuable post operative reeducation program.

- In $80 \%$ of cases the preoperative goals have been reached

- The satisfactory index is 6.35 on average

- The distance of gait was significantly increase in $65 \%$ of patients (from 65 to $750 \mathrm{~m}, \mathrm{p}<0.01$ )

- Shoe wearing was facilitated in $70 \%$ and splint no more used in $35 \%$

- Preoperative pain was present in $25 \%$ of patients and reduced from 8.5 to 1.8 on average on VAS

- Oral baclofen has been reduced from 45.5 to $5 \mathrm{mg} / \mathrm{day}(\mathrm{p}<0.01)$

Conclusions: Selective tibial neurotomy is a valuable neurosurgical procedure with long lasting effects to treat severe spastic foot. A functional improvement is obtained in most of the cases. A post operative rehabilitation program is mandatory to take benefit of surgery. 
Presentation Number: 120

FDG-PET Study of Cerebral Glucose Metabolic Changes After Thalamotomy in Patients With Essential Tremor

Takaaki Miyagishima, Gunma University, Maebashi, Japan; Masafumi Hirato, MD, PhD, Maebashi, Japan; Yuhei Yoshimoto, MD, $\mathrm{PhD}$, Maebashi, Japan

Introduction: Stereotactic thalamic ventralis intermedius (Vim) stimulation or thalamotomy was usually performed for the treatment of drug refractory essential tremor (ET). Though the effect of these treatments for ameliorating tremor was established, the real neural mechanisms are still unknown. In order to elucidate the mechanisms, we carried out FDG-PET study on the regional glucose metabolic rate before and after Vim thalamotomy.

Methods: Vim thalamotomy was indicated for 5 patients (4 males and one female) with drug refractory ET. Their mean age was 58.6 years-old (range, 26-74 years-old). FDG-PET study was performed before and within 2 weeks after surgery in all cases. Some images were flipped so that the treatment side could be stored on the same side. After that, we evaluated regional cerebral glucose metabolic changes using statistical parametric mapping (SPM).

Results: Clinically, tremor was abolished and tremor score was also improved in all cases. Comparing by SPM, the regional cerebral glucose metabolic rate reductions were found in thalamus on the ipsilateral to the side of surgery, and also in cerebellar hemisphere on the contralateral to operated side. Although it was not so obvious, a slight reduction was shown in the prefrontal cortex ipsilateral to the side of surgery.

Conclusions: FDG-PET study suggested the cerebellar metabolic activity decreased in accordance with diminishing tremor after Vim thalamotomy. The regional cerebral metabolic changes after surgery might reveal the functional change of the motor circuit including cerebello- thalamo- cortical pathway, which has been indicated to be implicated in the pathological neural mechanisms of ET. 
Session Title: Flash Session - Movement Disorders

Session Time: Tuesday, May 28, 2013, 3:30 pm - 4:30 pm

\section{Presentation Number: 85}

\section{Bilateral Caudal Zona Incerta Deep Brain Stimulation For Parkinson's Disease}

Patric Blomstedt, MD, PhD, Department of Neurosurgey, University Hospital of Northern Sweden, Umeå, Sweden; Jan Linder, MD, $\mathrm{PhD}$, Umeå, Sweden; Lars Forsgren, MD, PhD, Umeå, Sweden; Anna Fredricks, Umeå, Sweden; Gun-Marie Hariz, PhD, Umeå, Sweden; Marwan Hariz, MD, PhD, Umeå, Sweden

Introduction: The subthalamic nucleus is currently the target of choice in deep brain stimulation (DBS) for Parkinson's disease (PD), while thalamic DBS is used in some cases of tremor-dominant PD. Recently, a number of studies have presented promising results from DBS in the posterior subthalamic area, including the caudal zona incerta (cZi). The aim of the current study was to evaluate bilateral cZi DBS in patients who would otherwise have been eligible for STN DBS. Both patients with tremor dominant symptoms and non-tremor dominant symptoms were included. Here we present preliminary data from the patients evaluated one year after surgery.

Methods: 12 patients considered suitable for bilateral STN DBS were enrolled in the current study and implanted bilaterally in the cZi. The mean age was 59 years (range 49 - 70) and 2 were females. The patients were evaluated according to the motor part of the Unified Parkinson Disease Rating Scale (UPDRS) on/off medication before surgery and on/off medication and stimulation 12 months after surgery.

Results: The results on/off stimulation/medication are provided in the accompanying figure. At the follow-up in the offmedication state the total UPDRS III score improved by $52 \%$, tremor $90 \%$, rigidity $39 \%$ and bradykinesia $39 \%$, on stimulation as compared to off stimulation. The improvement on combined stimulation and medication was $71.5 \%, 99 \%, 66 \%$ and $60 \%$, respectively. In the 5 patients with dyskinesias the dyskinesia score was reduced from in mean 4.4 points to 0.8 (82\%). No serious adverse events occurred in this group of patients.

Conclusion: Bilateral cZi DBS seems to be safe and effective for patients with severe Parkinson's disease, especially regarding tremor. Head-to-head comparison is necessary in order to decide the role of cZi DBS in relation to STN DBS in advanced PD.

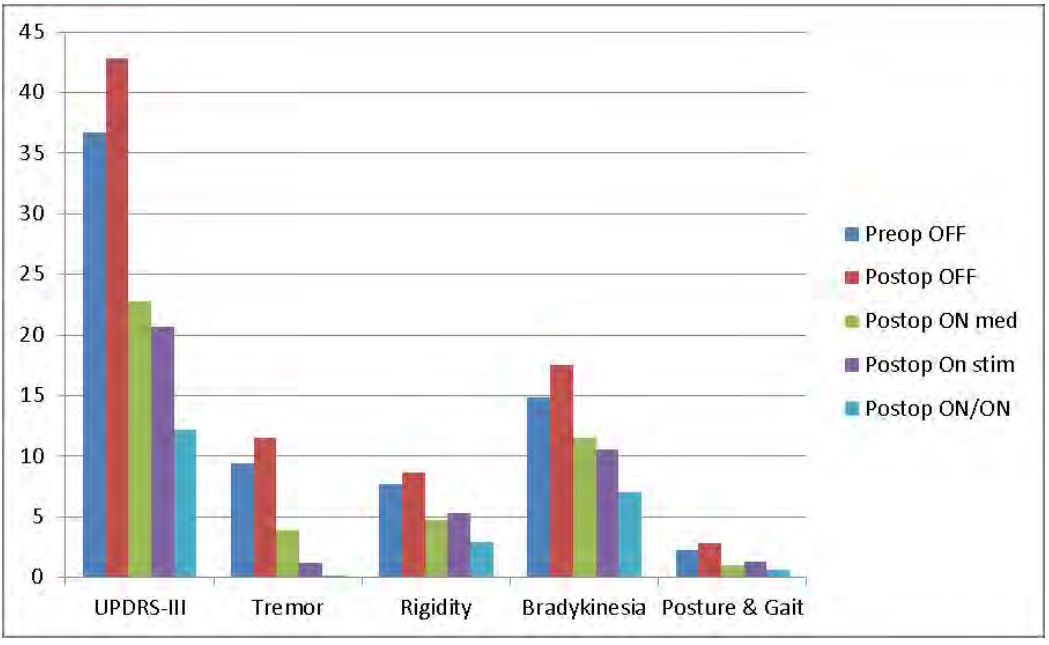


Session Title: Flash Session - Movement Disorders

Session Time: Tuesday, May 28, 2013, 3:30 pm - 4:30 pm

Presentation Number: 25

Effect of subthalamic nucleus stimulation during exercise on the mesolimbocorital dopaminergic region in Parkinson's disease: A positron Emission Tomography Study

Takao Nozaki, MD, Hamamatsu University School of Medicine, Hamamatsu, Japan; Kenji Sugiyama, MD, Hamamatsu, Japan; Shunsuke Yagi, MD, Hamamatsu, Japan; Etsuji Yoshikawa, BA, Hamamatsu, Japan; Toshihiko Kanno, RT, Hamamatsu, Japan; Tetsuya Asakawa, MD, Hamamatsu, Japan; Tae Ito, MD, Hamamatsu, Japan; Tatsuhiro Terada, MD, Hamamatsu, Japan; Hiroki Namba, MD, Hamamatsu, Japan; Yasuomi Ouchi, MD, Hamamatsu, Japan

Introduction: In order to elucidate the dynamic effects of deep brain stimulation (DBS) in the subthalamic nucleus (STN) during activity, we examined dopaminergic functions in the basal ganglia of patients with Parkinson's disease (PD) during motor exercise and STN-DBS.

Methods: Twelve PD patients who underwent STN-DBS operations at least 1 month prior, underwent 2 positron emission tomography scans during right foot movement in DBS-off and DBS-on conditions. To quantify motor performance changes, the motion speed and mobility angle of the foot at the ankle were measured twice. Estimations of the binding potential of [11C]raclopride (BPND) were based on the Logan plot method. Dopamine release analyses in basal ganglial subregions were performed with regions of interest and voxel-based methods.

Results: Significant motor recovery was found in the DBS-on condition. STN-DBS during exercise significantly reduced the $[11 \mathrm{C}]$ raclopride BPND in regions of the nonmotor loop of the basal ganglia-cortical circuit, including the caudate and the nucleus accumbens (NA), but not the dorsal or ventral putamen. The magnitude of dopamine release in the NA correlated negatively with the magnitude of motor load, indicating that STN-DBS facilitated motor behavior more smoothly and at less expense to dopamine neurons in the region. Moreover, the magnitude of the percentage of reduction in the motor scores of the UPDRS was significantly and inversely correlated with the \%reduction in the binding in the left caudate.

Conclusion: The lack of dopamine release in the putamen and the significant dopamine release in the ventromedial striatum by STNDBS during exercise suggested dopaminergic activation in the motivational circuit during action, resulting in a compensatory functional activation of the motor loop through direct or indirect pathways from the nonmotor to the motor loop system.

Effective stimulation by STN-DBS may feature less dopamine release in the region in conjunction with sufficient amounts of exercise. 
Presentation Number: 240

\section{Functional Connectivity Observed In Partial Epilepsy With Automotor Seizure}

Hiroki Nishibayashi, MD, PhD, Wakayama Medical University, Wakayama, Japan; Mitsuhiro Ogura, MD, PhD, Wakayama, Japan; Naoyuki Nakao, MD, PhD, Wakayama, Japan

Introduction: Automotor seizure is characterized by manual and oral automatism with impairment of consciousness, which frequently observed in temporal lobe epilepsy. It also appears as one of seizure manifestations in partial or generalized epilepsy. We investigated characteristics functional connectivity of epileptic brain showing automotor seizure using resting state functional connectivity magnetic resonance imaging(MRI).

Methods: Twenty two patients were examined. Of these, 7 showed automotor seizure, and 3 showed it as one of some different seizures. Brain structural and resting state functional images were acquired on a Philips 1.5-Tesla Achieva system. Functional data were acquired using a echo-planar sequence sensitive to blood oxygen level-dependent(BOLD) contrast with TR of $3000 \mathrm{~ms}$ for $5 \mathrm{~min}$ 30 s. Functional connectivity MRI analysis was performed using SPM8 and the conn toolbox. Entorhinal cortex was selected as the seed region of interest. Correlation coefficients were calculated between the average BOLD signal time series of the ROI and each voxel, and functional connectivity maps were created (correlation coefficient $>0.5$ ).

Results: Among 10 patients with automotor seizure, 6 with only it showed functional connectivity between entorhinal and orbitofrontal, or anterior cingulate area, although 3 with other seizure manifestations did not show similar findings. Among the patients without automotor seizure, only 2 showed functional connectivity between between entorhinal and orbito-frontal, or anterior cingulate area.

Conclusions: Epileptic patients showing automotor seizure may have different functional connectivity to those without it in the limbic system. These findings can explain the cognitive impairment or psychiatric disorder frequently observed in them. Resting state functional connectivity MRI may be utilized to disclose functional connectivity relating to automatism. 
Presentation Number: 107

\section{Posterior Cingulate Epilepsy: Clinical And Neurophysiological Analysis}

Rei Enatsu, MD, PhD, Cleveland Clinic, Cleveland, OH; Jorge Gonzalez-Martinez, MD, PhD, Cleveland, OH; Demitre Serletis, MD, PhD, Cleveland, OH; Juan Bulacio, MD, Cleveland, OH; Dileep Nair, MD, Cleveland, OH; Imad Najm, MD, Cleveland, OH; William Bingaman, MD, Cleveland, $\mathrm{OH}$

Introduction: Posterior cingulate epilepsy (PCE) is misleading because the seizure onset is located at an anatomically deep and semiologically silent area. This type of epilepsy is rare and has not been well described yet. Knowledge of the clinical and neurophysiological feature of PCE would be important for the interpretation of presurgical evaluation and better surgical strategy.

Methods: This retrospective analysis included seven intractable PCE patients. Six patients had post cingulate ictal onset identified on stereotactic electroencephalography (sEEG). One patient had post cingulate tumor. We analyzed clinical semiology, the scalp EEG/sEEG findings and cortico-cortical evoked potential (CCEP).

Results: The classifications of scalp EEG were various including non-localizible, lateralized to the seizure onset side, regional parieto-occipital, regional frontocentral and regional temporal. Five of seven patients showed motor manifestations including bilateral asymmetric tonic seizure, contralateral face tonic seizure and hypermotor seizure. In these patients, ictal activities spread to frontal (inferior frontal gyrus, supplementary motor area, anterior cingulate gyrus) and parietal areas (Precuneus, inferior parietal lobule, posterior cingulate gyrus, postcentral gyrus). Four patients showed alteration of consciousness (Dialeptic seizure or automotor seizure), in which seizure spread to medial temporal area or inferior parietal area. CCEP was performed in four patients and revealed the connection from the posterior cingulate gyrus to parietal, occipital, mesial temporal and posterior mesial frontal areas.

Conclusions: This study revealed the network from the posterior cingulate gyrus and the semiology of PCE (motor manifestation vs alterations of consciousness ) varies upon the seizure spread patterns. Scalp EEG was hard to localized the ictal onset area. 
Presentation Number: 103

\section{Extra-temporal Resection In Refractory Epilepsy}

Arthurt Cukiert, Clinica de Epilepsia de Sao Paulo, Sao Paulo, Brazil; Jose Burattini, Sao Paulo, Brazil; Cristine Cukiert, Sao Paulo, Brazil

Introduction: This paper reviewed a series of epileptic patients submitted to extra-temporal lobe resective surgery in the MR era. Methods: Four hundred and sixty-two patients submitted to extra-temporal epilepsy surgery from 1996 to 2012 were studied. Mean age at surgery was 18 years. Mean postoperative follow-up period was 5.7 years. One hundred and eighty-seven patients were submitted to temporal lobe, 187 to frontal, 70 to rolandic, 69 to posterior quadrant, 26 to parietal, 12 to occipital and 6 to insular cortical resection. Ninety-two patients were submitted to hemispherectomy.

Results: Eighty-two percent of the patients submitted to hemispherectomy have been rendered seizure-free. Ninety percent of the patients with MRI-positive frontal lobe have been rendered seizure-free; $61 \%$ of the patients with MRI-negative frontal lobe epilepsy have been rendered seizure-free. All patients submitted to occipital, parietal and insular resections were MRI-positive; $85 \%, 80 \%$ and $100 \%$ of them, respectively, have been rendered seizure-free after surgery. Sixty percent of the patients submitted to posterior quadrant resections were MRI-positive; $83 \%$ of them have been seizure-free after surgery. MRI-negative patients submitted to posterior quadrant resection did somewhat worse (67\% seizure-free). Four patients died: one patient had SUDEP immediately before leaving the hospital, 1 had malignant hyperthermia, 1 had malignant propofol syndrome, and 1 had malignant viscera phenitoin syndrome.

Conclusions: Better outcome was seen in patients with MRI-defined lesions. Morbidity was low, and those patients' deaths could not probably been prevented (1 SUDEP and 3 idiosyncratic syndromes). Overall, extra-temporal resection might be regarded as safe and effective. 
Presentation Number: 346

Efficacy of Vagal Nerve Stimulation in the treatment of Infantile Spasms: effects of different tuning and stimulation cycles. Andrea Landi, MD, San Gerardo Hospital, Monza, Italy; Daniele Grioni, MD, Monza, Italy; Andrea Trezza, MD, Monza, Italy; Leonardo Fiori, MD, Monza, Italy; Erik P. Sganzerla, MD, Monza, Italy

Introduction: Some reports focused the peculiar efficacy of VNS in the treatment of symptomatic infantile spasms (SIS). SIS are considered particularly dangerous for frequent falls - sometimes catastrophic - and for the poor sensitivity to AEDs; moreover, VNS seems to be the only effective treatment, although symptomatic. We report the efficacy of VNS in the treatment of SIS in a cohort of syndromic children and the effect of different stimulation settings.

Methods: 18 children affected by refractory epilepsy, not suitable for resective surgery, have been treated with VNS, since march 2006. Epilepsy was part of syndromic encephalic suffering, due to different aethyiologies. All patients showed severe mental retardation. Surgery was performed according to standard techniques. Output current was gradually increased to a maximum value ranging from 1,5 to $2 \mathrm{~mA}$. Duty cycle was changed according to different therapeutical requests. The efficacy of VNS was measured according to McHugh score, modelled on Engel's classification and adapted to VNS therapy.

Results: The most significant improvements were those concerning decrease of the frequency of seizures, mainly those causing falling to the ground, the lack of injuries in consequence of the seizures and the reduction of post ictal period. The mean global improvement in seizures severity scale was $+78 \%$. No patients developed adverse effects to VNS. Different intensities of stimulation and the fine tunig of duty cycle obtained improvements of the seizures control during the follow-up.

Conclusions: Our data, concerning the efficacy of VNS in the treatment of SIS are in agreement with previous reports. Since SIS does not recognize any effective pharmacological treatment and features as very dangerous - generally complicated with sudden falls -, the efficacy of VNS in decreasing the symptoms has to be considered like a first-line therapeutic tool. 
Presentation Number: 348

\section{Development Of Focal Brain Cooling System For The Treatment Of Intractable Epilepsy}

Masami Fujii, Yamaguchi University, Ube-Yamaguchi, Japan; Takao Inoue, PhD, Yamaguchi University, Japan; Sadahiro Nomura, MD, Yamaguchi University, Japan; Yuichi Maruta, PhD, Yamaguchi University, Japan; Yeting He, MD, Yamaguchi University, Japan; Hiroyasu Koizumi, MD, Yamaguchi University, Japan; Michiyasu Suzuki, MD, Yamaguchi University, Japan

Introduction: Limitations in medication and the neurosurgical indications for epileptic patients have motivated the design of implantable, device-based therapies. Focal brain cooling (FBC) is one of these options, because it has a potential to suppress epileptic seizures. The aim of our study is to establish the FBC system as a new neuromodulation therapy for patients with intractable epilepsy. Methods: Cooling devices with "Peltier" chips or water-cooling circuit systems were employed in animal and human studies. The cooling device was placed or implanted on the cortical surfaces in rats, cats and non-human primates. Kainic acid (KA) or penicillin G was then injected into the cortex to provoke epileptiform discharges (EDs). The cortices of the animals were cooled to varying degrees and the influence of FBC on EDs was investigated. Focal cooling was also applied in patients with intractable epilepsy. During surgery, cooling was performed on the epileptogenic cortices or hippocampi which had to be resected. Changes in the EDs, cerebral blood flow (CBF) and metabolites were investigated.

Results: The EDs and seizures were suppressed during cooling $\left(15-20^{\circ} \mathrm{C}\right)$ in animals. The neurophysiological functions were preserved during cooling above $15^{\circ} \mathrm{C}$ in rats and non-human primates. Histologically, no apparent damage was observed in the cortices of rats after cooling above $0^{\circ} \mathrm{C}$ for 1 hour. Intraoperative cooling to $15^{\circ} \mathrm{C}$ invariably showed the suppression of EDs, which was associated with a coupling of the $\mathrm{CBF}$ and the metabolism, with a significant reduction in the glutamate level in humans.

Conclusions: The present results support the physiochemical and technical feasibility of a cooling device-based therapy for epilepsy. 
Presentation Number: 100

Intra-operative Monitoring of Inter-hemispheric EEG Connectivity During Corpus Callosotomy

Masaki Iwasaki, MD, PhD, Tohoku University Graduate School of Medicine, Sendai, Japan; Eiichi Okumura, Sendai, Japan; Rie Sakuraba, Sendai, Japan; Izumi Itabashi, Sendai, Japan; Shin-ichiro Osawa, MD, Sendai, Japan; Kazutaka Jin, MD, PhD, Sendai, Japan; Nobukazu Nakasato, MD, PhD, Sendai, Japan; Teiji Tominaga, MD, PhD, Sendai, Japan

Introduction: Corpus callosotomy limits bilateral synchrony of epileptic discharges. However, no intra-operative measures have been implemented to evaluate physiological effect of callosal section. The present study investigated time-varying inter-hemispheric coherence in stages of corpus callosotomy.

Methods: Six patients who underwent total corpus callosotomy for medically intractable drop attacks were studied. Intra-operative scalp EEG was monitored simultaneously with surgical video. The time-varying inter-hemispheric coherence of the EEG was quantified by wavelet transform coherence (WTC) and trend analysis techniques (Mann-Kendall test and Sen's Slope estimator). The changes in the coherence were correlated with surgical steps of callosal section.

Results: The $4-13 \mathrm{~Hz}$ WTC decreased after corpus callsotomy for all patients. The significant trends of decreasing coherence were observed only during the posterior part of callosal section in three patients. Two patients presented significant trends though all stages of callosal section. One patient didn't present significant trend.

Conclusions: Decrease of inter-hemispheric coherence is not necessarily linear to the stages of callosotomy. The effect of corpus callosotomy is only maximized after complete section. Various manners of coherence drop suggest that the relative role of corpus callosum in bilateral synchrony is different between patients. Intra-operative evaluation of time-varying inter-hemispheric EEG coherence may be useful in monitoring the physiological effect of corpus callsotomy. 


\section{Presentation Number: 15}

Subtemporal Amygdalohippocampectomy For Medial Temporal Eplepsy. Operative Results And Neuropathological Findings. Tomokatsu Hori, Shinyurigaoka General Hospital, Kawasaki, Japan; Tomokatsu Hori, Shinyurigaoka General Hospital, Kawasaki, Japan; Tomokatsu Hori, Shinyurigaoka General Hospital, Kawasaki, Japan

Introduction: The role of medial temporal structure in the genesis of medically intractable epilepsy is well known. Especially if there is a definite hippocampal sclerosis by preoperative MRI, but if there is no definite sclerosis, the etiology of intractable temporal lobe epilepsy is the matter of debate. The role of amygdala, focal cortical dysplasia, tumor tissue, and perivascular oligodendrocytes in the genesis of intractable temporal lobe epilepsy will be discussed in this presentation from neuropathological findings of surgically resected specimen.

Methods: Surgery has been performed mainly by subtemporal approach or usual temporal lobectomy depending on the findings of preoperative work-up. The resected specimen include hippocampus, amygdala, other medial structures, and lateral temporal cortex was examined. In each case, neuropathological findings, postoperative seizure control (Engel's class), nuropsychological results, and postopertive FDG-PET findings were carefuly surveyed and analyzed.

Results: Hippocampal findings ( $\mathrm{n}=51)$ were classified into four types, Type 1: classical hippocampal sclerosis (HS) 61\%, Type 2: CA 1 sclerosis $2.4 \%$, Type 3: endofolium sclerosis $17.1 \%$, and finally no Hippocampal sclerosis $19.3 \%$. On the other hand, MRI findings $(\mathrm{n}=51)$ were classified as Typical HS $61 \%$, HS (-) $14 \%$, HIgh Intensity only $0.8 \%$, and hippocampal atrophy $17.6 \%$. In this presentation, the role of amygdala, hippocampal sclerosis, and presence of dysplastic cells, tumor tissue, and oligodendrocytes will be discussed in the genesis of intractability. Postoperative seizure control in total $77.5 \%$ and in Type 1 group, the result was the best. The true amygdala sclerosis was found in only one patient.

Conclusions: 1) subtemporal amygdalohippocampectomy will result postoperative improvement of neuropsychological scores and improved glucose metabolism at the side of resected hemisphere.

2) Various epilptogenic factors are contributory to the intractability including hippocampal sclerosis, amygdala gliosis, focal dysplastic tissue, and finally perivascular oligodendrocytes. 
Presentation Number: 16

\section{Risk Of Epilepsy After Traumatic Brain Injury: A}

Retrospective Population-based Cohort Study

Chien-Chang Liao, PhD, Taipei Medical University Hospital, Taipei, Taiwan

Introduction: Traumatic brain injury (TBI) is one of the most serious forms of trauma worldwide. Epilepsy causes significant functional disability among trauma survivors, and epilepsy after TBI is the most common cause of acquired seizure disorders in adults. We conducted a retrospective, population-based cohort study to examine the long-term risk of epilepsy in adult populations after TBI. Methods: Using Taiwan's National Health Insurance Research Database of reimbursement claims, we conducted a retrospective cohort study of 19336 TBI patients and 540322 non-TBI participants aged $\geq 15$ years as reference group. Data on newly developed epilepsy after TBI with 5-8 years' follow-up during 2000 to 2008 were collected. HRs and 95\% CIs for the risk of epilepsy associated with TBI were analysed with multivariate Cox proportional hazards regressions.

Results: Compared with the non-TBI cohort, the adjusted HRs of developing epilepsy among TBI patients with skull fracture, severe or mild brain injury were 10.6 (95\% CI 7.14 to 15.8$), 5.05$ (95\% CI 4.40 to 5.79 ) and 3.02 (95\% CI 2.42 to 3.77 ), respectively. During followup, men exhibited higher risks of post-TBI epilepsy. Patients who had mixed types of cerebral haemorrhage were at the highest risk of epilepsy compared with the non-TBI cohort (HR 7.83, 95\% CI 4.69 to 13.0). The risk of post-TBI epilepsy was highest within the first year after TBI (HR 38.2, 95\% CI 21.7 to 67.0 ).

Conclusions: The risk of epilepsy after TBI varied by patient gender, age, latent interval and complexity of TBI. Integrated care for early identification and treatment of post-trauma epilepsy were crucial for TBI patients. 


\section{A Novel Electrode Array for Electrocorticography of the Temporal Pole}

Taylor Abel, University of Iowa, Iowa City, IA; Mark Granner, Iowa City, IA; Hiroyuki Oya, Iowa City, IA; Matthew Howard, Iowa City, IA; Hiroto Kawasaki, University of Iowa, Iowa City, IA

Introduction: The temporal pole (TP), also known as Broadmann area 38, is frequently involved in the pathophysiology of temporal lobe epilepsy (TLE). Despite its importance in TLE, placement of corticography arrays over the TP is challenging due to its position in the middle cranial fossa. We set out to design and implement a specialized electrocorticography (ECoG) array to fit over the curvature of the TP.

Methods: A specialized ECoG array was designed to fit within the concavity of the middle cranial fossa in two sizes. The grids consist of 5 or 6 columns with the first 2 or 3 columns tapered such that they are composed of columns of 6-5-4-4-4 or 7-6-5-4-4-4 contacts for 23-contacts or 30-contacts grids, respectively. Computerized tomography was fused with MR imaging to demonstrate the precise localization of the electrode array over the temporal pole.

Results: Ten patients with medically intractable seizures were implanted with the electrode array as part of stage II monitoring for resection of epileptic foci. Imaging demonstrated the TP electrode array wrapping around the TP in each patient. Thirty percent of patients had a primary seizure focus localized to the TP. There were no complications directly associated with placement of the electrode array.

Conclusions: Our specifically designed TP ECoG electrode enables more comprehensive physiologic monitoring of the TP for the purpose of seizure localization and also research. In our preliminary experience, placement of the array is safe and useful for determining extent of resection in the surgery of the temporal lobe epilepsy.

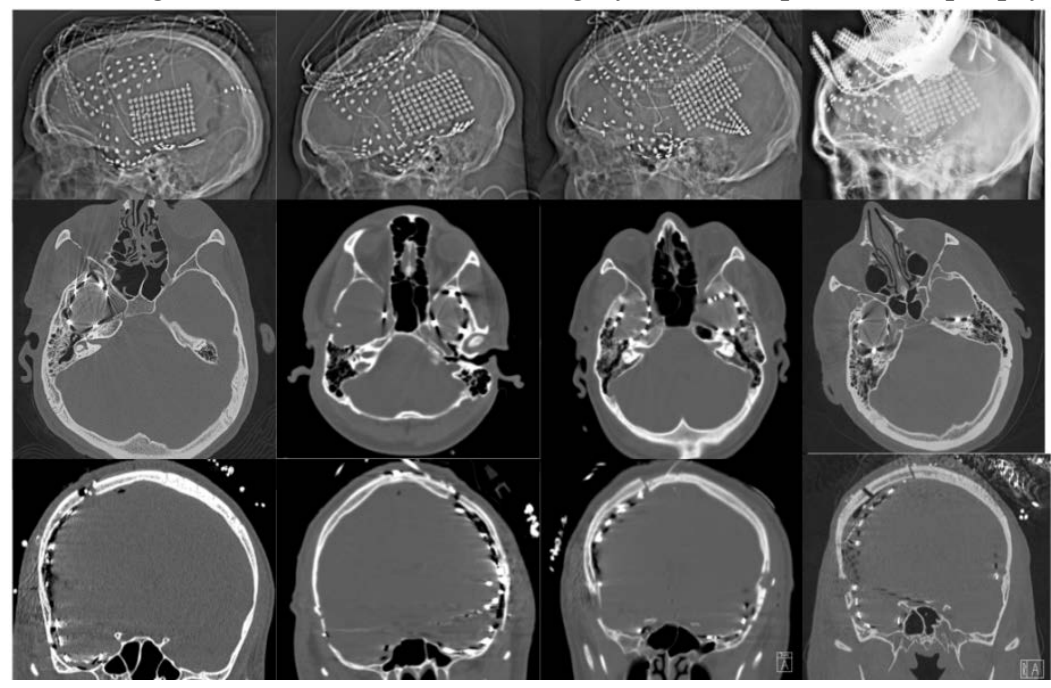


Presentation Number: 143

\section{Radiosurgery For Intractable Seizure Associated With Various Brain Disorders}

Yoshihisa Kida, Kamiiida Rehabilitation Hospital, Nogoya, Japan

Introduction: Non-invasive diagnosis and treatment of intractable seizure, associated with various brain lesions are reported.

Cases and methods: There are 8 cases of HH, 5 cases of MTLE and 27 cases of CM with gamma knife, those who were suffering from intractable seizures At radiosurgery, the side and location of the target volume are determined based on the findings of MEG. After the treatment, follow-up studies with MRI, EEG and MEG were done every 3 months and 6 months thereafter.

Results: Although it is often difficult to catch the seizure dipoles inside the HHs, secondary seizure foci are easily recorded over cerebral hemisphere. Seizure foci of MTLE are detected in ipsilateral temporal lobe with specific dipole cluster patterns. In cases of $\mathrm{CM}$, seizure dipoles are not localized inside the lesion, but in adjacent brain with an unpredictable distribution. The side and localization of seizure focus were correctly diagnosed with MEG for each disorder. MTLEs, $4.65 \mathrm{cc}$ in mean target volume, were treated with 20Gy at the margin, and resulted in Engel Class I (3 cases), and II ( 2 cases). HHs, 8-22 mm in mean diameter, were treated with a mean marginal dose of $18.5 \mathrm{~Gy}$. Small HHs with full tumor coverage responded well to GK, but large ones with partial coverage were not, resulting Engel' Class I in 3, and II in 3. The responses of CMs with intractable seizure, $16 \mathrm{~mm}$ in mean lesional diameter, treated with 17.2 Gy were not consistent. Seizures disappeared in 40.7\% (Class I) and improved in 29\%(Class II).

Conclusion: Non-invasive diagnosis and treatment for intractable seizure can be achieved with MEG and GK. Localization of seizure focus is crucial to achieve a good seizure control. A marginal dose more than $20 \mathrm{~Gy}$ or more is required for the control of intractable seizures. 
Presentation Number: 146

\section{Surgical Treatment Options For Adults With Lesional And-non Lesional Epilepsies}

Andrey R. Sitnikov, MD, The Federal Centre of Neurosurgery, Moscow, Russian Federation; Yuri Grigoryan, MD, Moscow, Russian Federation

Introduction: To present our approach and results of surgical treatment of patients with lesional and non-lesional epilepsies.

Methods: 83 patients with lesion-associated epilepsies underwent surgical resection with intra-operative corticography. Five patients with unremarkable MRI and bilateral epileptogenic foci received bilateral anterior thalamic stimulation.

Results: Among 36 patients with superficial brain tumours and cavernomas the radical resection was achieved in 28, subtotal resection in 7 and partial resection in 1 case. The epileptogenic area was identified in 27 cases. In group of 26 patients with cortical dysgenesias radical resection was done in 20 cases and subtotal resection in 6 cases. The epileptogenic area identified and resected in 23 cases. In the group of 21 patients with subcortically located lesions a radical resection achieved in 13 and subtotal resection in 8 cases. In the 1st group Engel's 1 outcome have 28 patients, Engel's 2 - 4, Engel's 3 - 2 and no improvement - 2 patient. The similar results we found in the 2nd group. In the 3rd group the seizures control was achieved by tumour removal without an additional cortex resection. All 5 patients with non-lesional epilepsy have bilateral epileptic foci in temporal or frontal lobes. In four cases we used the microelectrode recording for electrode placement. The stimulation parameters were $130 \mathrm{~Hz}, 4 \mathrm{~V}, 90-\mu \mathrm{s}$ pulse-width, cycling $1 \mathrm{~min}$ on $/ 5$ min off. Treatment showed a significant decrease in seizure frequency, with a mean reduction of $74 \%$ (mean follow-up, 17 months). Four of the patients had a seizure reduction more than $85 \%$.

Conclusions: The intra-operative corticography with resection of ictal or continuous epileptogenic discharges improve the surgical outcome in lesion-associated epilepsies. DBS of the anterior thalamus is a comparatively safe procedure and highly effective in patients with medically resistant seizures who are not eligible for resective surgery. 
Presentation Number: 407

Outcome After More Than Two Year Of Temporal Lobe Epilepsy Surgery With Stereoeeg

Taku Ochiai, Ochiai Neurological Clinic, Saitama, Japan; Yuichi Kubota, Saitama, Japan; Tomokatsu Hori, Tokyo, Japan

Introduction: We shows our outcome after more than two year of temporal lobe epilepsy surgery with StereoEEG.

Methods: We evaluated 22(M: 11/F: 11) cases who were decided a temporal lobe epilepsy using MRI, VideoEEG, PET, SPECT and semiology.

Average age were 32.6(14-53) and average follow-up period was 50.1(26-83)months. All patients had CPS and four showed GTC also. We performed stereoEEG in order to decide the resection area.

Results: 7 cases(31.8\%) were mesial type temporal lobe epilepsy and we resected mescal temporal structure(GroupA). $15 \operatorname{cases}(68.2 \%)$ were mesial and lateral type temporal lobe epilepsy and we resected lateral temporal lobe in addition to mescal temporal structure also(GroupB).

Seizure free was 4case(57.1\%) in GroupA and 14cases(93.3\%) in GroupB. 2case(28.6\%) were medication free in GroupA and 9 case $(60.0 \%)$ in GroupB.

Conclusions: Mesial type temporal lobe epilepsy with SEEG was only $31.8 \%$ in our cases. Even if the patient had CPS and Hippocampal sclerosis, an epileptogenic zone located not only mesial temporal structure but also lateral temporal lobe.

We achieved more than $80 \%$ seizure free and more than $50 \%$ medication free. 


\section{Presentation Number: 304}

\section{Fractionated Stereotactic Radiosurgery for Intracranial Metastases: Tumor Control}

Joel S. Katz, D.O., Manhasset, NY; Marina Kushnirsky, Hofstra North Shore-LIJ School of Medicine, Manhasset, NY; Jonathan P. Knisely, M.D., Manhasset, NY; Maged Ghaly, M.D., Manhasset, NY; Michael Schulder, M.D., Hofstra North Shore-LIJ School of Medicine, Manhasset, NY

Introduction: Fractionated SRS may confer radiobiologic treatment advantages in the eradication of metastatic brain tumors. We compared the results of single and fractionated SRS for patients with metastatic tumors.

Methods: We reviewed all patients from our institution who were treated with SRS for intracranial metastases between January 2010 and January 2013. Collected data included diagnosis, tumor location, lesion volume, and SRS dose. Local control (LC) and volume changes after fractionated or single fraction SRS were compared using Wilcoxon rank sum and t-test.

Results: 152 patients with 294 lesions underwent SRS. 211 lesions were available for follow-up with serial MRI from 0.2 -31.8 months ( $8.77 \pm 7.57$ months) after SRS, with overall LC of 71.6\%. 53 lesions had treatment volumes greater than $3 \mathrm{cc}$. Of these, 26 lesions underwent single session SRS and 27 were treated in 3 sessions. Median prescription doses for single and fractionated SRS were $20 \mathrm{~Gy}$ and $24 \mathrm{~Gy}$, respectively. Tumor progression was observed during follow-up in 3/26 lesions treated with a single session, compared with progression in $1 / 27$ lesions treated with 3 sessions $(\mathrm{p}=0.02$, see fig 1$)$. Overall, lesion volume following fractionated SRS decreased by $73.4 \%$, vs. a $62.2 \%$ decrease after single session SRS $(\mathrm{p}=0.0005)$.

Conclusions: In patients with metastatic tumors greater than $3 \mathrm{cc}$ in volume, fractionated SRS yielded LC better than that obtained with single session SRS. In these patients, the volumetric decrease in tumor size was greater after multiple fraction than single fraction SRS. We recommend consideration of fractionated SRS for patients with metastatic tumors larger than $3 \mathrm{cc}$.

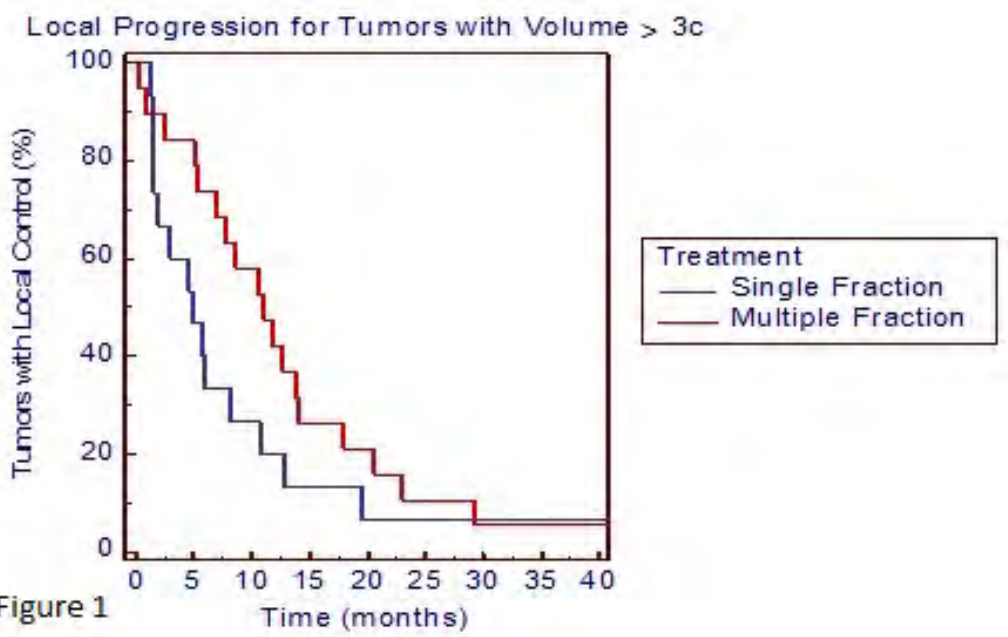


Session Title: Flash Session - Radiosurgery

Session Time: Tuesday, May 28, 2013, 3:30 pm - 4:30 pm

Presentation Number: 117

\section{Magnetic Resonance Diffusion-weighted Imaging for Targeting Glioma in Stereotactic Biopsy: A Correlative Study with Histopathological}

Examination and Clinical Outcome

Yi-Chieh Hung, MD, Taipei Veterans General Hospital, Taipei, Taiwan; Huai-Che Yang, MD, Taipei, Taiwan; Kang-Du Liu, Taipei, Taiwan; Sanford P.C. Hsu, MD, Taipei, Taiwan; David Hung-Chi Pan, MD, Taipei, Taiwan; Wan-Yuo Guo, MD, PhD, Taipei, Taiwan

Introduction: The underestimation of astrocytoma grade frequently occurs. We aimed to improve the diagnostic accuracy of astrocytoma by integrating the analysis of the MR diffusivity of tumor tissues for biopsy targeting.

Methods: The study included 26 astrocytoma patients diagnosed by stereotactic biopsy. Morphological MR diffusion-weighted imaging (DWI) data were retrieved for evaluating the targeting accuracy. The histopathology results showed that 6 were low-grade (grade II) and 20 were high-grade (grade III and IV) astrocytomas. During the 3-year follow-up, 3 of the low-grade astrocytoma patients remained tumor progression-free, and the other 3 deteriorated, converting to a clinically high-grade status. Thus, 23 tumors were defined as high grade by the study endpoint. A quantitative method of directly measuring the signal intensity of astrocytomas in DWI was used. All signal intensity measurements of tumors were normalized by the white matter at the contralateral-frontal lobe, and defined as the biopsy target signal intensity ratio (BIR) and the highest signal intensity ratio (HIR). The correlations between the BIR and the histopathological diagnosis and that between the HIR and the clinical outcome were evaluated.Results: The BIRs of histopathological high-grade were higher than those of the low-grade astrocytomas $(P<.01)$. A BIR cutoff value of 1.6 permitted optimal discrimination between low-grade and high-grade astrocytomas, with a sensitivity of $83 \%$ and a specificity of $100 \%$.

Conclusions: DWI is a helpful imaging tool for tissue characterization of astrocytomas. It may be used to guide stereotactic biopsy for obtaining representative tissue samples for histopathological diagnosis and for clinical monitoring when tumor accessibility is limited.
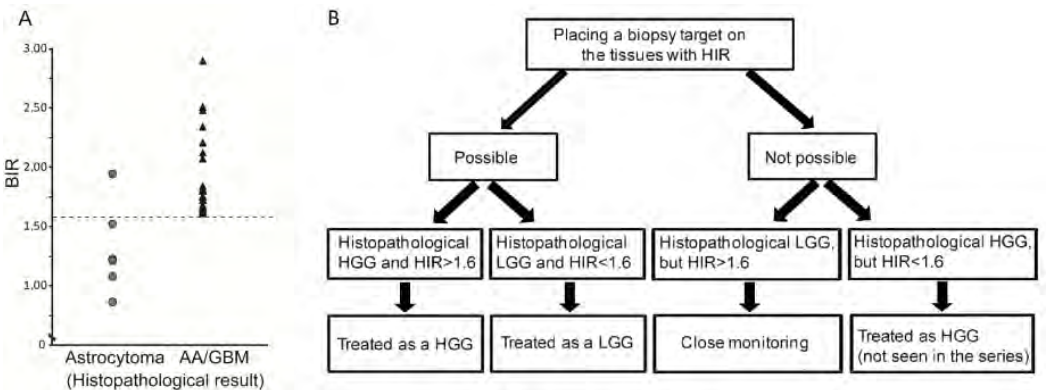
Radiographic Evolution of Thalamotomy Lesions Generated by Stereotactic Radiosurgery: A Two-Year Follow-up Anand I. Rughani, MD, University of Toronto, Toronto, ON, Canada; Suneil Kalia, MD, PhD, Toronto, ON, Canada; Andres M. Lozano, MD, PhD, Toronto, ON, Canada; Mojgan Hodaie, MD, Toronto, ON, Canada

Introduction: In treating tremor, thalamic lesions are variously generated by radiofrequency-produced thermal lesions, stereotactic radiosurgery, and now focused ultrasound. How radiosurgical lesions evolve temporally has not been clearly established, and is the focus of this analysis.

Methods: We reviewed the T1 and T2-weighted MRIs of patients undergoing radiosurgical thalamotomy. Volume was calculated on $\mathrm{T} 1$ and $\mathrm{T} 2$ images by the formula $4 / 3 *(\pi) *(\mathrm{X} / 2) *(\mathrm{Y} / 2) *(\mathrm{Z} / 2)$, where $\mathrm{X}$ is the lateral diameter, $\mathrm{Y}$ is the antero-posterior diameter, and $\mathrm{Z}$ is the height of the lesion.

Results: Twenty-seven patients were treated with gamma-knife thalamotomy, with essential tremor $(\mathrm{n}=17)$ as the most common diagnosis treated, followed by tremor-dominant Parkinson's Disease $(n=4)$. The mean patient age was 76-years-old (range 45-89). Twenty patients had at least one post-treatment MRI, and 17 of whom had serial imaging (range 7-54 months). Ten patients had at least two-year follow-up imaging. The average lesion volume on T1 was $26.5 \mathrm{~mm}^{3}$ at 6 months, $33.1 \mathrm{~mm}^{3}$ at 12 months, and $34.1 \mathrm{~mm}^{3}$ at 24 months. The average lesion volume on T2 was $251.0 \mathrm{~mm}^{3}, 150.3 \mathrm{~mm}^{3}$ and $90.6 \mathrm{~mm}^{3}$ at the same time points. Nine of 17 patients showed increasing lesion volume on T1 between the first and second MRI, while only 5 of 17 patients showed increased lesion volume on T2 between the first and second MRI. Patients with clinical improvement did not demonstrate larger lesion volumes compared to patients without clinical improvement on 12-month T1W (43.8 vs. $25.2 \mathrm{~mm}^{3}$; $\mathrm{p}=0.163$ ) or T2W MRI (127.2 vs. 169.6 $\left.\mathrm{mm}^{3} ; \mathrm{p}=0.354\right)$.

Conclusions: Lesion volume appears to increase on T1W MRI up to about one-year post-treatment, and then stabilize, whereas the volume of T2 signal decreases from 6 months onward. Patients with clinical improvement had a trend toward greater T1 lesion volumes compared to those with suboptimal clinical result.
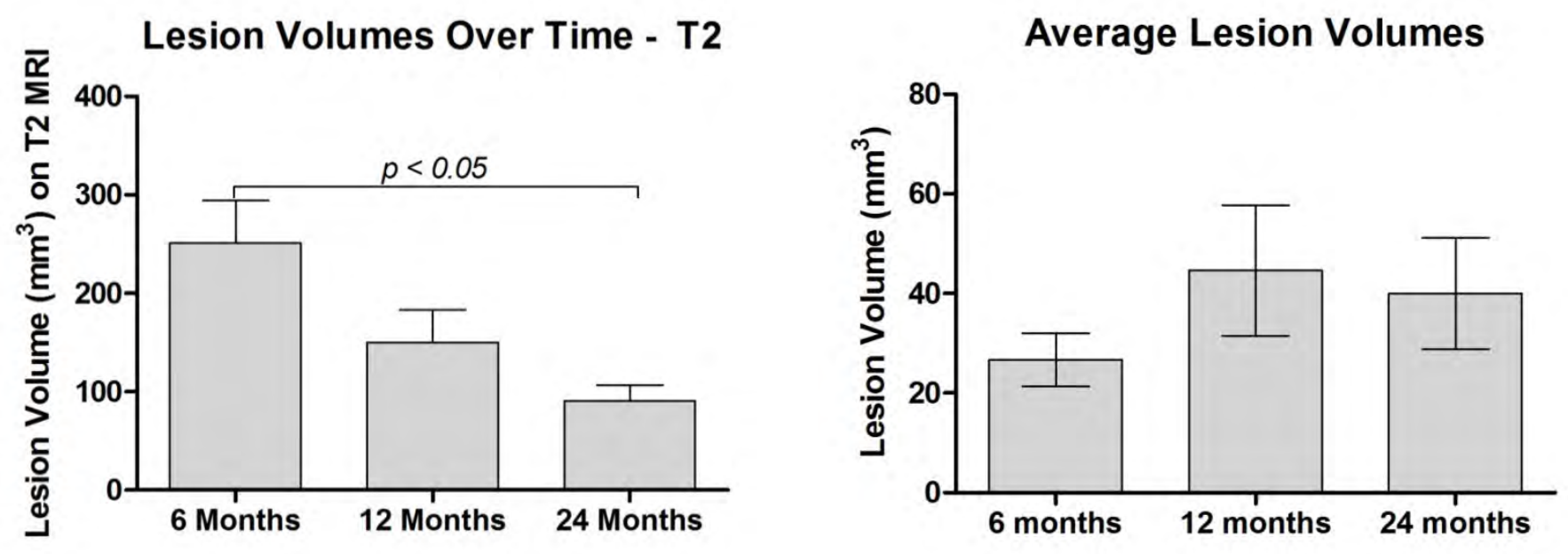
Interstitial Brachytherapy With Iodine-125 Seeds For Low Grade Brain Stem Gliomas In Adults: Diagnostic And Therapeutic Intervention In A One-step Procedure

William O. Contreras Lopez, MD, University Freiburg Medical Center, Freiburg im Breisgau, Germany; Michael Trippel, MD, Freiburg im Breisgau, Germany; Thomas Reithmeier, MD, Freiburg im Breisgau, Germany

Purpose: To report on iodine-125(I125) interstitial irradiation in the treatment of low grade brain stem gliomas in adults.

Patients and Methods: 10 patients with well-circumscribed lesions of the brainstem and histological confirmation of low grade glioma treated with stereotactically implanted I-125 seed in our department between 1995 and 2012 were retrospectively analyzed.

Results: In nine patients the lesion was treated with one I-125 seed and in one patient, two spatial separated lesions were implanted, therefore a total of $11 \mathrm{I}-125$ seeds were implanted. The mean volume of the 11 lesions was $2.76 \mathrm{ml}$ (range: $0.5-7.2 \mathrm{ml}$ ), mean activity of the seeds was $6.23 \mathrm{mCi}$ (range: $1.5-11.1 \mathrm{mCi}$ ), mean duration of irradiation was 28.5 days (range: $21-41$ days) and mean effective dose rate was $9.16 \mathrm{cGy} / \mathrm{h}$ (range: $6.2-12 \mathrm{cGy} / \mathrm{h}$ ). The 30 days perioperative morbidity and mortality rate was $0 \%$. Median follow up was 72.5 month (range 5-168 months). Six of ten patients were free of progression until last follow up.

Conclusion: In our experience at the University Clinic in Freiburg Germany, interstitial radiosurgery based on MRI is a safe and effective method to diagnose and treat low grade gliomas of the brain stem. Furthermore randomized studies are needed to confirm the therapeutic impact of this method in comparison to external beam radiation of brain stem gliomas.
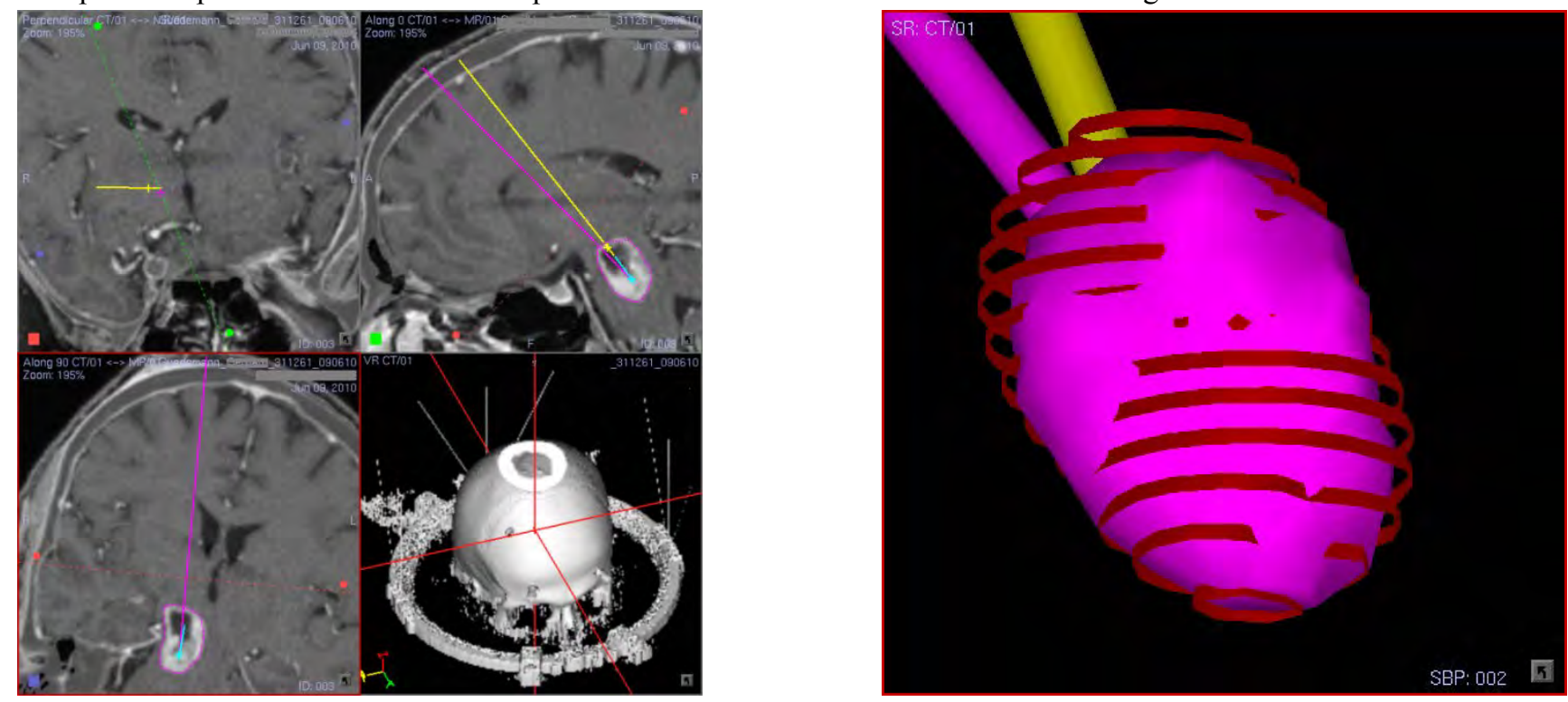
Presentation Number: 245

\section{Long term outcomes of gamma nife Surgery For Classic Trigeminal Neuralgia In Patients Younger Than 50 Years} Jung Kyo Lee, Asan Medical Center, Seoul, Korea, Republic of

Introduction: Gamma knife surgery (GKS) could be as an alternative, minimally invasive treatment in medically refractory trigeminal neuralgia (TN) although microvascular decompression (MVD) has historically been the treatment of choice for TN in young patient. We evaluated the long-term results of GKS in the treatment of TN patients younger than 50 years.

Methods: From 2004 to 2010, 20 cases of classic trigeminal neuralgia in younger than 50 years were treated with GKS. The radiosurgical target was on the petrous bone in retrogasserian portion. The maximal radiation doses ranged from 75 to $90 \mathrm{~Gy}$. The median age of the patients was 43.9 years (range 34-49 years), and the median time from diagnosis to GKS was 55 months (range 12180 months). The median follow up was 79 months (range 25-105 months).

Results: During follow up period, all patients reported initial pain relief and the pain was controlled in all patients (100\%, BNI I-III) while 13 patients (65\%) achieved pain free state without medications (BNI I). BNI score of I-III was found in all cases except one 95\%) during follow up 25-105 months. One patient was lost the follow up after recurrence at 28 months. There are only mild facial dysfunction (BNI facial numbness score II) in 4 patients (20\%).

Conclusion: GKRS could be a reasonable treatment option for young patients unwilling or unable to undergo more invasive surgical approaches. It yields durable pain control in a majority of patients, as well as improved quality of life with limited complications. 
Presentation Number: 259

\section{A Preliminary Result Of The Prospective Control Study Of Gamma Knife Radiosurgery For Trigeminal Neuralgia With One Or Two Isocenters}

Peng Li, west china hospital, sichuan university, chengdu, China; Wei Wang, chengdu, China

Introduction: A prospective randomized control study of Gamma Knife radiosurgery for trigeminal neuralgia was carried out to investigate the different results between one isocenter and two isocenters.

Methods: Between June 2010 and January 2012, 54 patients were divided into two groups based on the randomized rule. Single isocenter was delivered to patients of Group I and two isocenters to Group II patients. The central dose was limited to $85 \mathrm{~Gy}$ or $90 \mathrm{~Gy}$ for each patient of both groups. Follow-up was established through the telephone visit.

Results: Fifty four patients were followed up with an average time of 26.6 months. There were 28 patients in Group I and 26 patients in Group II. At last follow-up, the number of complete pain relief, partial pain relief and no effect was $8+5(46.4 \%), 6+7(46.4 \%)$ and $2(7.1 \%)$ in Group I, and 12 (46.2\%), 5+6 (42.3\%), 3(11.5\%) in Group II respectively. The mean time to pain relief was 5.66 weeks of Group I patients and 5.35 weeks of Group II patients. Radiation induced brain injury was observed in two patients of Group I. Facial numbness was reported by one Group I patient and two Group II patients. No pain recurrence was reported.

Conclusions: There is not enough evidence for the benefit with two isocenters based on the initial result of this study. A central radiation dose of $90 \mathrm{~Gy}$ might increase the risk of radiation induced brain injury. However, the preliminary result of these 54 patients was limited. More cases and longer follow-up are needed. 
Presentation Number: 255

\section{Gamma Knife Radiosurgery as a complementary treatment of large vestibularis schwannomas}

Etienne Holl, Medical University Graz, Graz, Austria; Karin Pistracher, Graz, Austria; Markus Hoffermann, Graz, Austria; Frank Unger, Graz, Austria

Introduction: Three different major treatment options can be offered in patients with large vestibularis schwannomas (VS); microsurgery, radiosurgery and combined treatment. Microsurgery including intraoperative monitoring is the gold-standard treatment for large VS. But patients with major medical problems or complications during the operation can alternatively or complementary be treated with radiosurgery.

Methods: To examine those 3 options, 90 patients with large VS (Diameter $\geq 2.5 \mathrm{~cm}$ ) were retrospectively assessed within 12 years. Twenty-seven patients underwent microsurgery, thirty-nine were treated by Gamma Knife Radiosurgery (GKRS) and 24 patients received both, mirco- and radiosurgery. The mean age of all patients was 52 years; the mean age of the patients treated with GKRS (60 years) was higher than the other groups. There was no significant difference in gender distribution in the three groups. The mean follow up was 40 months.

Results: Reduction of size of tumor was statistically significant in all three groups but expectedly more impressive after microsurgery and combined therapy than after GKRS. Damaging of the facial nerve with clinical relevance (House-Brackman $\geq$ III) was significantly higher in the microsurgical (26\%) group than in the GKRS-group $(0 \%)$ and combined group ( $0 \%)$, respectively. The rate of severe complications was $22 \%$ after microsurgery, $13 \%$ after combined treatment and $0 \%$ after GKRS. Other complications as imbalance and mild trigeminal pain were found in $4 \%$ of the patients after GKRS and showed continuous improvement during follow up.

Conclusions: Microsurgery was associated with a greater rate of facial neuropathy with clinical relevance and other complications like CSF-leakage or infection. Combined treatment by tumor reduction and GKRS treatment of the residual neuroma can reduce complication rate for large tumors. GKRS for VS having a diameter $\geq 2.5 \mathrm{~cm}$ was found to be a complementary or alternative treatment method with less risk for severe complications. 
Presentation Number: 257

\section{Staged Gamma Knife Radiosurgery for Relatively Large Metastatic Brain Tumors}

Atsuya Akabane, MD, Gamma Knife Center, NTT Medical Center Tokyo, Tokyo, Japan; Itaru Kanazawa, MD, Tokyo, Japan; Chikayuki Ochiai, MD, Tokyo, Japan

Introduction: In gamma knife radiosurgery (GKS) for metastatic brain tumors (METs), as the tumor volume increases, the expected local tumor control rate decreases. Therefore, we performed staged GKS for relatively large METs that are difficult to manage by conventional GKS. In this paper, we report preliminary results of the staged GKS.

Methods: We retrospectively analyzed 26 patients who underwent staged GKS at our hospital since 2007 for METs with a tumor volume of more than $8 \mathrm{ml}$. The median age of the patients was 66 years (range, 41-86 years) and the median tumor volume at initial GKS was $10.6 \mathrm{ml}$ (range, 8.9-28.3 ml). The staged GKS was principally performed 3 times every 2 weeks (3-staged group). The median prescription and central dose in each treatment were $10 \mathrm{~Gy}$ (range, 8-10 Gy) and $15 \mathrm{~Gy}$ (range, 11-18 Gy), respectively. In 9 patients with a survival period of 6 months or less, staged GKS was performed 2 times in a 2-week interval to shorten the treatment period (2-staged group). In these patients, the median prescription and central dose in each treatment were 13 Gy (range, 12-14 Gy) and 21 Gy (range, 19-26 Gy), respectively. The median follow-up period was 6 months (range, 1-29 months).

Results: The tumor volume at the final treatment in the 3-staged group and the 2-staged group showed an average reduction of $34 \%$ and $19 \%$, respectively. In the 17 symptomatic patients, 11 patients $(65 \%)$ showed a rapid improvement in the Karnofsky performance score within the treatment period (2-4 weeks). The median survival time was 10.5 months, and local tumor control was achieved in 18 patients (70\%). Symptomatic radiation-induced adverse effect was not observed.

Conclusions: Although our results are preliminary, they suggest that staged GKS may be effective for relatively large METs even in the case of advanced cancer patients. 
Presentation Number: 341

\section{Long Term Results of Gamma Knife Radiosurgery for Craniopharyngioma}

Huai che Yang, Taipei Veterans General hospital, Taipei, Taiwan

Introduction: GK radiosurgical results for the treatment of craniopharyngioma

Material and Method: Between 1993 and 2011, a total of 132 patients with craniopharyngioma had 159 GKS at the Taipei Veterans General Hospital. 113 patients had more than one year regularly follow-up and are included in our series. In our series, 47 patients were female and 66 patients were male. 82 patients $(72 \%)$ had previous treatment including craniotomy, trans-sphenoidal endoscopic surgery, or Radiation before GKS. 13 patients (12\%) had only cyst aspiration or shunt procedure before GKS and 18 patients (16\%) had no previous treatment history before GKS. Our median tumor volume was $5.6 \mathrm{ml}(0.2-27.6 \mathrm{ml})$. Our peripheral dose to the tumor ranged from 10-16Gy (Median 12Gy), with maximum doses ranged 15.4-32Gy (Median 21.8Gy). Follow-up time was ranged from 12-230 months (Median 41 months). Data on clinical and imaging changes after radiosurgery were analyzed.

Results: Tumor progression free survival rate after once Gamma knife treatment is $85 \%$ in 2 years $\mathrm{f} / \mathrm{u}, 72 \%$ in 5 years $\mathrm{f} / \mathrm{u}$ and $38 \%$ in 10 years f/u. 9 patients died during follow-up but only 4 patients' (4\%) mortality caused by symptom related to tumor progression. Only one patient was found to have a mildly restricted visual field after Gamma knife; no additional endocrinological impairment or neurological deterioration could be attributed to the treatment.

Conclusion: Multimodality management of patients with craniopharyngiomas seemed to provide a better quality of patient survival and greater long-term tumor control. It is suggested that GKS accompanied by adjuvant stereotactic procedures should be used as an alternative in treating recurrent or residual craniopharyngiomas if further microsurgical excision cannot promise a cure. 
Presentation Number: 148

\section{Proposed Mechanism And Treatment Strategy For Cyst Formation And Enlargement Following Gamma Knife Surgery For} Arteriovenous Malformation

Takashi Shuto, MD, Yokohama Rosai Hospital, Yokohama, Japan

Introduction. We retrospectively studied the mechanism of cyst formation and enlargement after Gamma Knife surgery (GKS) for arteriovenous malformation (AVM).

Methods. Twenty-five patients who developed cyst formation following GKS for AVM, 15 male and 10 female patients aged 14 to 48 years, were retrospectively identified among 775 patients who underwent GKS for AVM at our institution.

Results. So-called expanding hematoma was associated with the cyst in 9 patients. The nidus volume at GKS was 0.1-36 ml, and the prescription dose was 18-28 Gy. Complete obliteration of the nidus was obtained in 18 patients, and partial obliteration in 7. Cyst formation was detected from 1.1 to 15 years after GKS. Thirteen patients underwent craniotomy, including two patients in whom the incompletely obliterated nidus was removed simultaneously, and two received placement of Ommaya reservoir. Spontaneous regression of cyst was observed in one patient. Serial magnetic resonance imaging was performed in the other patients because the cyst size was stable or asymptomatic. Histological examination of the cyst wall revealed linear hemosiderin deposits with gliosis, and the nodular lesion, enhanced on magnetic resonance imaging, contained granulation tissue with chronic hemorrhage from newly developed capillary vessels.

Conclusion. Cysts developing after GKS for AVM enlarge mainly due to repeated minor hemorrhage from a reddish nodular angiomatous lesion developing within the adjacent brain, suggesting that the optimal treatment is wide opening of the cyst with removal of the associated angiomatous lesion by craniotomy. 
Presentation Number: 132

\section{Hydrocephalus}

following Gamma Knife Radiosurgery in the Vestibular Schwannoma.

Kyoung-Hyup Nam, Pusan National University Hospital, Pusan National University School of Medicine, Busan, Korea, Busan, Korea, Republic of; Won-Ho Cho, MD,PhD, Busan, Korea, Republic of; Seung-Heon Cha, MD,PhD, Busan, Korea, Republic of; Chang-Hwa Choi, MD,PhD, Busan, Korea, Republic of

Introduction: With the introduction of stereotactic radiosurgery, gamma knife radiosurgery (GKRS) has become a popular alternative to open surgery in the vestibular schwannomas(VS). However, there has been little attention to the adverse effect of GKRS.

Hydrocephalus (HD) may be a complication of GKRS in VS, although the direct relationship is still under debate.

Methods: Between March 2004 and October 2010, 78 patients with VS were treated by GKRS, 5 patients have shown HD. We present five cases with HD following GKRS in VS and discuss both the incidence and the risk factors of HD associated with GKRS in VS.

Results: Four of the patients presented here developed symptomatic HD from the preexisting ventriculomegaly, and one patient presented newly onset HD. All patients suffering from HD had large tumors (diameter $\geq 20 \mathrm{~mm}$ ). Four of them showed increased protein concentration in the CSF. All patients who developed HD underwent ventriculoperitoneal shunt. In our cases, we suggested that both the tumor size and the CSF protein concentration might be predictive factors of HD following GKRS in VS.

Conclusions: We demonstrated the relatively high incidence of HD following GKRS. Tumor size, CSF protein levels and pre-existing ventriculomegaly were proposed as risk factors of HD following GKRS in VS. GKRS is likely to exacerbate the development of HD in VS. The progression of HD should be monitored closely following GKRS, particularly in patients with large VS. 
Presentation Number: 64

\section{Gks For Cervical Spinal Cord Tumors}

Mooseong Kim, MD, Ph.D, Inje University Busan Paik Hospital, Busan, Korea, Republic of

Introduction: Foramen magnum, upper cervical spinal cord area are complex area. We have treated and assessed the effect of GammaKnife radiosurgery for the tumors of these areas.

Methods: We have treated 13 patients with GKS during last 18 years. The 13 patients were comprised of 3 men and ten women, their ages ranging from 26 to 75 years(mean 50.06 years). The diagnoses were 2 hemangioblstomas, 4 meningiomas, 4 astrocytomas, 1

hemangioma, 1 ependymoma, 1 hypoglossal tumor, etc. Surgical intervention was performed in 4 before radiosurgery. Mean maximal dose was $21.3 \mathrm{~Gy}$, mean marginal dose $10.6 \mathrm{~Gy}$.

Results: Mean follow up period was 37.1 months(8-142 months). Eight tumor had markedly decreased in size on follow up MRI.The other cases were stationary. There was no complication.

Conclusions: The result of this study suggests that GKS is effective for upper cervical tumor, foramen magnum tumor without neurological deficit.

Key Words: Foramen magnum, cervical cord, GammaKnife 
Presentation Number: 162

\section{Clinical Application of the Verification Method of Leksell GammaPlan in Multiple Metastatic Brain Tumors} Beong Ik Hur, PhD, Pusan National University Hospital, Busan, Korea, Republic of; Byung Kwan Choi, MD, PhD, Busan, Korea, Republic of; Won Ho Cho, MD, PhD, Busan, Korea, Republic of; Seung Heon Cha, MD, PhD, Busan, Korea, Republic of

Introduction: Leksell Gamma Knife ${ }^{\circledR}$ Radiosurgery(LGKRS) is based on a single-fraction high dose treatment strategy. Therefore, the independent verification for the planning results of Leksell GammaPlan ${ }^{\circledR}$ (LGP) is very important for ensuring patient safety and minimizing the risk of treatment errors. Although several verification techniques have been previously developed and reported, no method has ever been tested statistically on multiple LGK target treatments. The purpose of this study was to perform and evaluate the accuracy of a verification method (Modified Variable Ellipsoid Modeling Technique, MVEMT) for multiple target treatments.

Methods: A total of 500 locations in 10 consecutive patients with multiple metastatic brain tumor targets were included in this study. We compared the data from an LGP planning system and MVEMT in terms of dose at random points, maximal dose points, and target volumes. All data was analyzed by t-test and the Bland-Altman plot, which are statistical methods used to compare two different measurement techniques.

Results: No statistical difference in dose at the 500 random points was observed between LGP and MVEMT. Differences in maximal dose ranged from $-2.4 \%$ to $6.1 \%$. An average distance of $1.6 \mathrm{~mm}$ between the maximal dose points was observed when comparing the two methods.

Conclusions: Statistical analyses demonstrated that MVEMT was in excellent agreement with LGP when planning for radiosurgery involving multiple target treatments. MVEMT is a useful, independent tool for planning multiple target treatment that provides statistically identical data to that produced by LGP. Findings from the present study indicate that MVEMT can be used as a reference dose verification system for multiple tumors. 
Presentation Number: 334

\section{Complications Of Radiosurgery For Tic In Patients With MS}

Keith Gomes, MBBS, FRACS, University of Calgary, Foothills Hospital, CALGARY, AB, Canada; Layth Mula-Hussain, MD, MSc, CALGARY, AB, Canada; Robert Nordal, MD, FRCPC, CALGARY, AB, Canada; Zelma Kiss, MD PhD FRCSC, CALGARY, AB, Canada

Introduction: Patients with trigeminal neuralgia (tic) from multiple sclerosis (MS) are harder to manage and recur earlier after surgery than those with idiopathic tic. Stereotactic radiosurgery (SRS) has emerged as a reasonable treatment option for this group, although results are not as good as those in the non-MS population. What is not known and what we sought to determine here was whether MS tic pain is more likely to get worse after SRS, than non-MS tic pain.

Methods: Our study is a retrospective cohort study comparing patients with tic and MS to those without MS who underwent SRS treatment. Nineteen patients (8 MS, 11 non-MS) with at least 6-months follow-up have undergone SRS in our centre. The clinical histories were reviewed by a clinician, uninvolved in the patients' care, who assigned a Barrow Neurological Institute (BNI) score for each clinic visit. Patients were divided into those whose scores worsened or improved, and a Chi square statistic with one degree of freedom was derived for the 3- and 6-month follow-up for each group.

Results: At 3-month review, 4 of 19 patients had a worse BNI pain score than pre-SRS. Three of these patients had MS. At 6-month review, all the non-MS patients demonstrated improved BNI scores, whereas 4 patients in the MS group had worsened (Chi square $=6.97, \mathrm{p}=0.008$ ). No significant differences in the BNI anaesthetic scores were noted at either the 3- or 6-month reviews. Conclusions: Tic patients with MS are more likely to worsen in the 6 month time period post-SRS than those without MS. This suggests that the inflammatory response to SRS in the MS population may persist for several months and worsen tic pain. MS patients undergoing SRS should be warned about this possible complication. 
Presentation Number: 178

\section{Validity Test of the Modified Recursive Partitioning Analysis for Brain Metastases Cases from Breast Cancer Treated} Radiosurgically

Osamu Nagano, MD,PhD, Chiba Cardiovascular Center, Ichihara, Japan; Toru Serizawa, MD,PhD, Tokyo, Japan; Yasunori Sato, $\mathrm{PhD}$, Chiba, Japan; Yoshinori Higuchi, MD,PhD, Chiba, Japan; Junichi Ono, MD,PhD, Ichihara, Japan; Naokatsu Saeki, MD,PhD, Chiba, Japan

Introduction: We verified the validity of the Modified

Recursive Partitioning Analysis (m-RPA), categorized into three sub-classes (RPA I+IIa, IIb and IIc+III, Yamamoto et al, Int J Radiat Oncol Biol Phys 2012;83: 1399-1405) from another subset of breast cancer brain metastasis (BM) patients.

Methods: Our IRB-approved database of the Gamma Knife House, Chiba Cardiovascular Center and the Tokyo Gamma Unit Center, Tsukiji Neurologic Clinic, including 268 breast cancer BM patients treated with gamma knife radiosurgery(GKS) during 1998-2011 periods.

Results: Two hundreds sixty six females and 2 males were included. Mean age was 57 [range; 27-90] years. Mean and median lesion numbers were 12 and 4, respectively, range one to 50. Cumulative tumor volumes ranged from $0.01 \mathrm{cc}$ to $100.0 \mathrm{cc}$, the median being $8.5 \mathrm{cc}$. Patient numbers of RPA class I, II and III were 24 (9.0\%), 202 (75.3\%) and $42(15.7 \%)$. Among 202 class II patients, the patient numbers were 30, 80 and 92 in the classes IIa, IIb and IIc respectively. Median survival times were 25.6 months in class $\mathrm{I}+\mathrm{IIa}(95 \% \mathrm{CI}$; 19.8-29.7), 14.3 in class IIb (95\% CI; 11.6-16.8, p=.0088 vs. class(I+IIa), 6.4 in class IIc+III (95\% CI; 5.2-7.5, p<.0001 vs. class IIb). This new system showed highly statistically significant differences among the three sub-classes ( $\mathrm{p}<.01$ for all subclasses), and 95\% CIs had no overlaps.

Conclusions: We conclude that this new grading system, the m-RPA, is applicable to different subset of breast cancer BM patient groups and should be considered when designing future clinical trials involving BM patients. 
Presentation Number: 190

\section{Deep Brain Stimulation for Parkinson Disease - Results of the EARLYSTIM Study}

Maximilian H. Mehdorn, MD, PhD, University of Kiel, Kiel, Germany; Philippe Cornu, M.D.,Ph.D., Paris, France

Deep Brain Stimulation has become the accepted surgical treatment of choice for a variety of movement disorders, particularly Parkinson's Disease (PD), and has been evaluated in a number of trials. It has been suggested that patients may benefit also earlier in their disease course then usually thought. In order to test this hypothesis, a German-French working group initiated in 2006 a binational multicenter prospective randomized parallel-group controlled study including idiopathic Parkinson's disease patients at an early stage of the disease. A total of 251 patients (age $52 \mathrm{ys,} \mathrm{disease} \mathrm{duration} 7.5 \mathrm{ys}$ ) were randomized for early STN stimulation or best medical treatment (BMT) and followed for 2 years, Quality of Life being the primary endpoint with many additional aspects considered. PDQ-39-SI improved by $26 \%$ in the stimulation group and remained unchanged in the best medical treatment group. UPDRS-III without medication, UPDRS-II in the worst state and UPDRS-IV improved 53\%, 30\% and 61\% respectively and all significantly more than the BMT. Subthalamic stimulation was superior to medical therapy in Parkinson's disease with early motor complications. The study was funded by the German Ministry of Research ('Klinische Studien' 01KG0502), and the French "Programme Hospitalier de Recherche Clinique National" (P050909). Medtronic co-funded the study. 


\section{Presentation Number: 71}

\section{Treatment Of Memory Decline In Alzheimer'S Disease By Deep Brain Stimulation: A Feasibility Study.}

Denys Fontaine, MD, PhD, University Hospital of Nice, Nice, France; Audrey Deudon, CHU de Nice, CMRR, Nice, France; Jean Jacques Lemaire, Clermont-Ferrand, France; Jacques Darcourt, Nice, France; Philippe Robert, CHU de Nice, CMRR, Nice, France

Introduction: Recent studies have suggested that memory circuits can be modulated by deep brain stimulation (DBS). This propriety might be used to slow down the cognitive decline of patients suffering from Alzheimer's Disease (AD). We conducted a prospective study to evaluate the feasibility and safety of DBS in AD patients with mild cognitive decline.

Methods: Inclusion criteria were: patients ( $<70$ years old) with AD diagnosed for less than 2 years, predominant impairment of episodic memory, and Mini Mental Status (MMSE) between 20 and 24. The fornix was stimulated bilaterally by electrodes implanted stereotactically in the hypothalamus. Clinical, biological, neuropsychological and imaging evaluations were conducted 3 months before surgery and 3, 6 and 12 months thereafter.

Results: During the one year-period of inclusion, 110 patients with recently diagnosed AD and predominant impairment of episodic memory were screened. Only 9 patients (eligibility rate $8,2 \%$ ) fulfilled all the inclusion criteria. Finally, only one patient accepted to be operated (acceptance rate 11,1\%) and completed the study. No complications occurred and the stimulation was perfectly tolerated. After 1.5 year of stimulation, the memory scores (MMSE, ADAS-Cog, Free and Cued Selective Reminding Test) were stabilized compared to baseline, and mesial temporal lobes metabolism significantly increased.

Conclusions: This pilot study provides new data about the safety of fornix DBS in the hypothalamus. However, it suggests that only a small proportion of $\mathrm{AD}$ patients might be concerned by this approach and that the acceptance of DBS by AD patients was low, rising questions about the relevance of this approach to meet the expectations of these patients. 
Presentation Number: 227

\section{Pedunculopontine nucleus-stimulation in patients with Advanced Parkinson's disease}

Mohammad J. Naushahi, BMedSci, MRCS, Imperial College Neuromodulation Group, London, United Kingdom; Dipankar Nandi, DPhil, FRCS (SN), London, United Kingdom

Introduction: Stimulation of the subthalamic nucleus (STN) has been in routine use in the treatment of patients with advanced Parkinson's disease (PD), refractory to medical therapy. In recent years, pedunculopontine nucleus (PPN)-stimulation has been explored to address the axial motor symptoms of gait freezing and loss of postural control. However, the role of PPN-stimulation remains unclear. This study explores the effect of PPN-stimulation in reducing falls, improving gait and postural control in patients with advanced PD.

Methods: A prospective, four-phase, within-subject cross-over, and double-blinded study evaluating the effect of medication only v bilateral STN v bilateral PPN v concomitant bilateral STN \& PPN-stimulation on axial motor symptoms of advanced PD. Patients and carers maintained a Falls \& near-Falls Diary and contemporaneous pedometer records of mobility. The posture and gait components of the Unified Parkinson's Disease Rating Scale (UPDRS) were recorded. The Timed Get-Up \& Go Test, The Berg Balance Scale and Quality of Life (PDQ-39, EQ-5D \& SF-36) assessments were also applied.

Results: A significant reduction in freezing of gait $(\mathrm{p}<0.01)$ and a improvement in activities of daily living (ADL; UPDRS Part II) (p $<0.001)$ is achieved with PPN-stimulation alone at $1.5 \pm 0.5 \mathrm{~V}, 25 \pm 5 \mathrm{~Hz}, 60 \mu$ s and in combination with STN-stimulation at $2.5 \pm$ $0.5 \mathrm{~V}, 140 \mathrm{~Hz}, 60 \mu \mathrm{s}$, as compared to ON medication alone. Neurophysiological findings are to be reported separately. PPNstimulation induced momentary ipsilateral oscillopsia at commencement on $\geq 2.0 \mathrm{~V}$ with rapid habituation, consistent with previous findings. No other complications were reported with PPN-stimulation.

Conclusion: Concomitant bilateral STN \& PPN-stimulation offer the possibility of ameliorating the axial motor symptoms of advanced PD. 
Presentation Number: 173

\section{Long Term Effects of Deep Brain Stimulation of the Medial Forebrain Bundle (sIMFB DBS) for treatment resistant depression}

Volker A. Coenen, MD, Stereotactic and Functional Neurosurgery, Freiburg University Neurocenter, Freiburg, Germany; Bettina Bewernick, PhD, Department of Psychiatry, Bonn University, Germany; Sarah Kayser, MD, Department of Psychiatry, Bonn University, Germany; Burkhard Maedler, PhD, Stereotactic and Functional Neurosurgery, Freiburg, Germany; Thomas E. Schlaepfer, MD, Department of Psychiatry, Bonn University, Germany

Introduction: Treatment-resistant major depressive disorder (TR-MDD) is a prevalent and debilitating condition. Deep Brain Stimulation (DBS) to different targets has been proposed as a putative treatment.

Methods: In this pilot study we assessed safety and efficacy of DBS to the supero-lateral branch of the medial forebrain bundle (sIMFB) in seven patients with highly refractory depression. Primary outcome criterion was severity of TR-MDD as assessed with the Montgomery Åsberg Depression Rating Scale (MADRS). General psychopathologic parameters, social functioning, and tolerance were assessed with standardized scales, the Global Assessment of Functioning (GAF) scale, quality of life (SF-36) and neuropsychological tests.

Results: Six patients attained the response criterion; response was rapid, mean MADRS of the whole sample was reduced by more than $50 \%$ at day seven after onset of stimulation. At last observation (12 to 33 weeks), six patients were responders; among them four were classified as remitters. Social functioning (GAF) improved in the sample as a whole from serious to mild impairment. Mean stimulation current was $2.86 \mathrm{~mA}$; all side effects (strabismus at higher stimulation current, one small intracranial bleeding during surgery, infections at the IPG site) could be resolved at short-term.

Conclusions: These preliminary findings suggest that bilateral stimulation of the slMFB may significantly reduce symptoms in TRMDD. At the time of presentation at WSSFN Tokio 2013, we will have analysis of long term stimulation results over a period of 7088 weeks of stimulation. This protocol is registered at ClinicalTrials.gov with the identifier NCT01095263. 


\section{Presentation Number: 27}

\section{Deep Brain Stimulation of the Subcallosal Cingulate Area for Treatment-Refractory Anorexia Nervosa: Phase I Pilot Trial} Nir Lipsman, MD, University of Toronto, Toronto, ON, Canada; Blake Woodside, MD, Toronto, ON, Canada; Peter Giacobbe, MD, MSc, Toronto, ON, Canada; Clement Hamani, MD, PhD, Toronto, ON, Canada; Andres M. Lozano, MD, PhD, Toronto, ON, Canada

Introduction: Anorexia Nervosa (AN) is a common condition affecting primarily young-adults and is associated with the highest mortality rate of any psychiatric illness. Current treatments, including pharmacologic and psychosocial approaches, have proven ineffective in a large proportion of patients. There is mounting evidence that mood and anxiety play important roles in the maintenance of AN and that Deep Brain Stimulation (DBS) can be a safe and effective means of modulating activity within circuits mediating such symptoms. We provide evidence that DBS can be used safely in patients with AN, and that chronic stimulation can lead to improvements in mood, anxiety, and ultimately, a change in the natural history of the illness.

Methods: We conducted a phase I, pilot trial of subcallosal cingulum (SCC) DBS in six patients with chronic, treatment-refractory AN (mean age: 38; duration of illness: 18.3 years; baseline BMI: 13.7). Patients were identified through Canada's largest eating disorders program, and approved by the multidisciplinary team prior to enrollment. Subjects underwent baseline medical and psychological examination as well as neuroimaging, repeated at 6-months after surgery.

Results: At 9-months post-operatively, 3/6 (50\%) patients were at a BMI significantly higher than their historic baseline. Changes in BMI were accompanied by reductions in depressed mood, improvements in anxiety, and reductions in AN-related preoccupations and rituals. Functional imaging performed at 6-months post-DBS showed significant metabolic changes in regions of the brain implicated in AN, consistent with a reversal of known baseline abnormalities.

Conclusions: DBS in a group of highly refractory AN patients, was associated in some patients with a significant change in the natural history of their illness. Improvements in psychological symptoms were associated with subsequent changes in body weight, as well as local and remote changes in cerebral metabolism. Additional, larger studies are required to verify the results of this pilot trial. 


\section{A Randomized Blinded Trial of Nucleus Accumbens Ablation to Treat Opiate Dependence in Humans: Location Correlates with Outcome}

Guodong Gao, MD,PhD, Tangdu Hospital, Xi'an, China; Xuelian Wang, Xi'an, China; Nan Li, Xi'an, China; Shunnan Ge, Xi'an, China; Li Gao, Xi'an, China

Introduction: Opiate addiction remains intractable in a large percentage of patients. Multiple studies identify a central role of the nucleus accumbens (NAc) in addiction; several studies note decreased addictive behavior after interventions in this brain area. In China, ablation of the NAc was common before November 2004, though it was poorly regulated. This study, a prospective randomized double-blinded trial carried out from January 2004 to November 2004, investigates the effect of different stereotactic lesions within NAc on opiate addiction to maximize benefit and minimize risk.

Methods: 78 opiate-dependent patients were treated at the Tangdu Hospital Fourth Military Medical University during Jan. 2004 to Nov. 2004 with bilateral stereotactic radiofrequency lesioning of the posteromedial one third of NAc. Patients were randomly divided into four groups (A, B,C and D). Relative to the initial target, the lesion in Group A was enlarged laterally and superiorly, in Group B anteriorly and superiorly,and in Group C laterally and anteriorly. Among these three groups, the lesion volume was identical. In Group $\mathrm{D}$, the lesion extended laterally and anteriorly and superiorly. Using opiate abstinence as the primary endpoint, four years of follow-up data for all patients regarding relapse and complications were collected. This trial is supplementarily registered with Current Controlled Trials, number ISRCTN33954423.

Results: Four patients were withdrawn at the fourth year post-operation. For convenience of interpretation, this article appointed these patients as relapses. The overall abstinence rate was 53.8\% (42/78). The abstinence rates of Group C (75.0\%) and Group D (76.2\%) were higher than Groups A (31.3\%) and B (28.6\%) (p $\leq 0.005)$. The neuropsychological adverse event rate was $24.3 \%(18 / 74)$ and did not differ statistically between the four groups.

Conclusions: Although sometimes accompanied by neuropsychological adverse events, stereotactic ablation of NAc may effectively treat opiate addiction. Lesion location significantly impacts treatment efficacy.
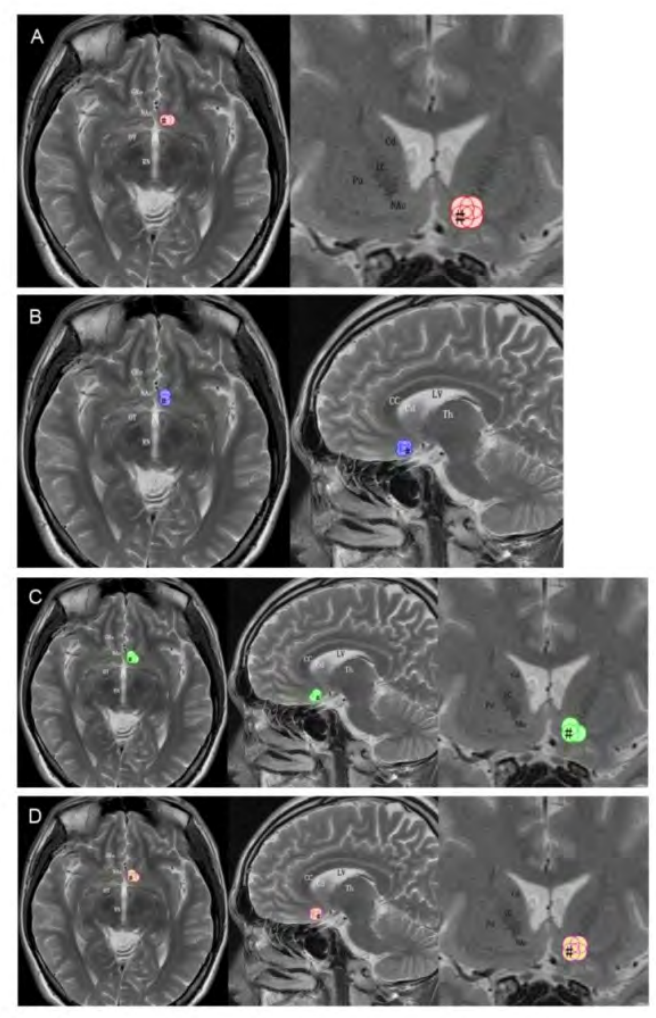

Opiate Abstinence

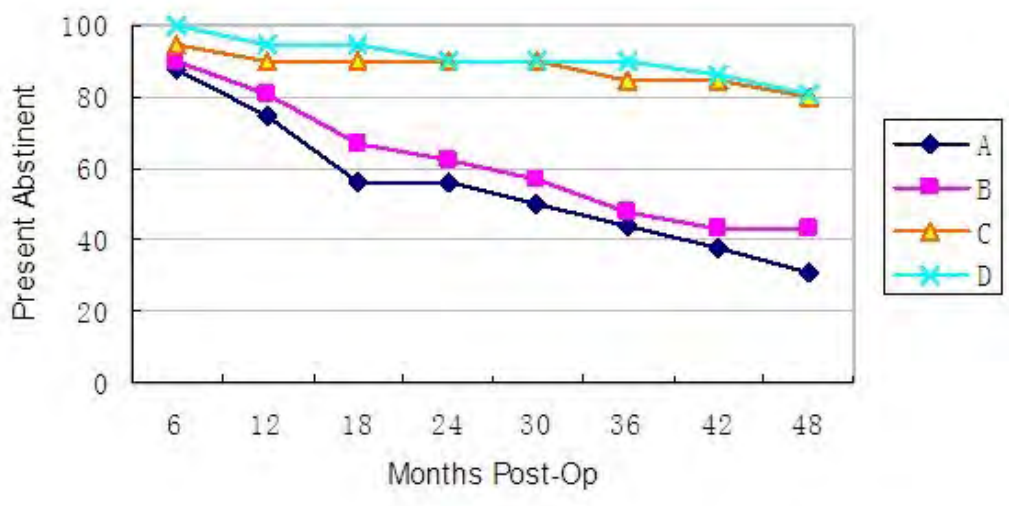




\section{What Happened in the Lobotomized Brain? Tractographic Analysis of Prefrontal lobotomy for Mental Illness}

Katsushige Watanabe, MD,PhD, Dept. of Neurosurgery, Tokyo Metropolitan Matsuzawa Hospital, Tokyo, Japan; Ayako Isoo, MD,PhD, Tokyo, Japan; Kazuhiro Niizato, MD,PhD, Tokyo, Japan; Toshihiro Nonaka, MD,PhD, Tokyo, Japan; Takeshi Inui, MD, Tokyo, Japan; Ryousuke Nakamura, MD, Tokyo, Japan; Akira Masuda, MD, Tokyo, Japan; Tetsuya Hino, MD, Tokyo, Japan; Kayo Ishimoto, MD, Tokyo, Japan; Ikuko Kayama, MD, Tokyo, Japan; Yuuji Okazaki, MD,PhD, Tokyo, Japan; Makoto Taniguchi, $\mathrm{MD}, \mathrm{PhD}$, Tokyo, Japan; Masahiko Saito, MD,PhD, Tokyo, Japan

Introduction: Although prefrontal lobotomy was an obsolete remedy for treatment-refractory mental illness, some patients who have undergone lobotomy continue to reside in psychiatric hospitals. The surgery achieved a certain therapeutic response, however, that concomitantly produced several complications of lobotomy, such as personality defect (Miller and Psych, 1967), because this extensive lesion was often associated with impairment of frontal lobe function. In view of this, the purpose of this study was to identify fiber connectivities severed by the prefrontal lobotomy.

Methods: Diffusion tensor imaging (DTI) scans were acquired from 7 healthy adults, from 3 schizophrenia patients and from 3 prefrontal lobotomized patients with schizophrenia, group-matched for age, on a 1.5T scanner. All lobotomized patients underwent the surgeries approximately more than 40 years previously. Voxelwise statistical analysis of the fractional anisotropy (FA) data in white matter tracts were carried out by using Tract-Based Spatial Statistics (TBSS) to compare between schizophrenia patient group and healthy control subject group, and between lobotomized schizophrenia group and nonlobotomized schizophrenia patient group.

Results: Nonlobotomised schizophrenia patient group had lower FA than healthy control group in white matter fiber tracts of genu and splenium of corpus callosum, anterior limb of internal capsule and superior longitudinal fasciculus. However, compared with nonlobotomized schizophrenia group, lobotomized schizophrenia group had lower FA in the white matter of ventromedial prefrontal lobe, rostral and dorsal region of corpus callosum and cingulum arch.

Conclusion: The result indicates that the connectivity sacrificed by the prefrontal lobotomy is largely divergent, which involves the non-overlap area with the white matter tracts impaired in schizophrenia. Shared and distinct patterns of connectivity between them may explain surgical effects; instead of narrowing in the capacity to adapt to changing life situations, functioning well in terms of social activities as long as external circumstances were stable. 
Presentation Number: 332

\section{Where The Parallels Meet: Evidence Of Functional Convergence Of Basal Ganglia Circuits In Subthalamic Nucleus From Intra-operative Multichannel-microelectrode Recording}

Erich T. Fonoff, MD, PhD, University of Sao Paulo, Sao Paulo, Brazil; Edgar Morya, PhD, Sao Paulo, Brazil; Koichi Sameshima, MD, PhD, Sao Paulo, Brazil; Manoel J. Teixera, MD, PhD, Sao Paulo, Brazil; Miguel A. Nicolelis, MD, PhD, Natal, Brazil

Introduction: Behavioral changes in PD patients after STN DBS in the same subjects who experienced significant motor improvement suggest that the greatest convergence of different circuits is reached within STN. The present study approaches this hypothesis by multiunit recording during microelectrode mapping of subthalamic area.

Methods: Twelve consecutive PD patients underwent multiunit recording through a 32-channel-microelectrode based in an assembly of microwires used for electrophysiological mapping of STN during therapeutic implantation of DBS electrodes for standard

dorsolateral STN target. After recognition of typical STN activity, the patient was asked to perform simple tasks. Neuronal signal recording was time-locked with the presentation of emotional images (IAPS Image Bank), a decision-making task and movement of the contralateral hand. The signal was submitted to off-line spike-sorting analysis and correlated to behavioral responses to the threemodality tasks.

Results: The electrode set-up allowed outstanding signal-to-ratio neuronal recording that permitted STN electrophysiological activity pattern recognition as well as in routine microelectrode recording. Eight of the twelve patients were able to perform all the tasks during the procedure. Over all, 276 neurons were studied

(average 30,66 7,599 per patient). Most of the neuron firing were related to the motor task (54,9\%). Interestingly, other 16,7\% were active during decision-making period of cognitive task, while $19,8 \%$ of the neurons were only responsive to the positive and negative emotional valence images randomly presented. We have also observed that $16,5 \%$ of all neurons were multimodal (responsive to all the tasks).

Conclusion: Although, the target aimed for therapeutic purpose was the dorsolateral part of STN, substantial neuron activity was related to limbic and cognitive information processing. This data is consistent with the hypothesis the basal ganglia modulates motor activity as complex behavioral series that are strongly influenced by the cognitive and emotional context in which they are executed. 
Session Title: Papers Session - Best Science II

Session Time: Wednesday, May 29, 2013, 10:50 am - 12:00 noon

Presentation Number: 276

\section{Experimental Comparison In Non-human Primates Of Focal Tolerance Between Epidural And Subdural Implantation Of A Cortical Neuroprosthesis}

Napoleon R. Torres-Martinez, MD PhD, CEA Clinatec, Grenoble, France; David Ratel, PhD, Grenoble, France; Fabien Sauter, PhD, Grenoble, France; Cecile Moro, PhD, Grenoble, France; Darlene Lobel, MD, Mayo Clinic, MN; Alim Louis Benabid, MD PhD, Grenoble, France

Introduction: With the emergence of chronic application of cortical Neuroprostheses in the field of brain computer interface (BCI), focal tolerance and ideal degree of contact between the brain surface and electrodes has emerged as a major problem. To address this issue, a study was designed to compare the focal tolerance of a prototype BCI Neuroprosthesis (BCI-N) in the subdural and epidural space.

Methods: We created a $25 \mathrm{~mm}$ BCI-N, based on our clinical implant, adapted for a non human primate (NPH) skull. 2 healthy Macaca Rhesus were implanted with 3 BCI-N and one single $25 \mathrm{~mm}$ electrode matrix as control. 2 BCI-N were implanted in the subdural space and 1 in the epidural space in each subject. NHPs were observed during 3 and 6-month periods, respectively, and behavioral tests were conducted during that period. Histology was obtained at the end of the study.

Results: NHPs tolerated the implantation well, with no significant side effects. Subdural implantation showed development of reactive tissue that re-formed the duramater in both NPHs. In the 3-month contact subject, neoformed dura had non-statistically significant different thickness compared to native dura. In the 6-month subject, this neoformed dura had not grown further and did not differ histologically from reactive tissue in the epidural site. No neuronal degeneration or glial reactivity was seen in the cortex of either site. Conclusion: Subdural cortical implantations of BCI-N in primates produced a neoformed dura, with no difference in thickness than native dura after 3 months of contact. This suggests that the benefit obtained from ECOG signals using subdural vs. epidural grids could be lost in long-term implantation.

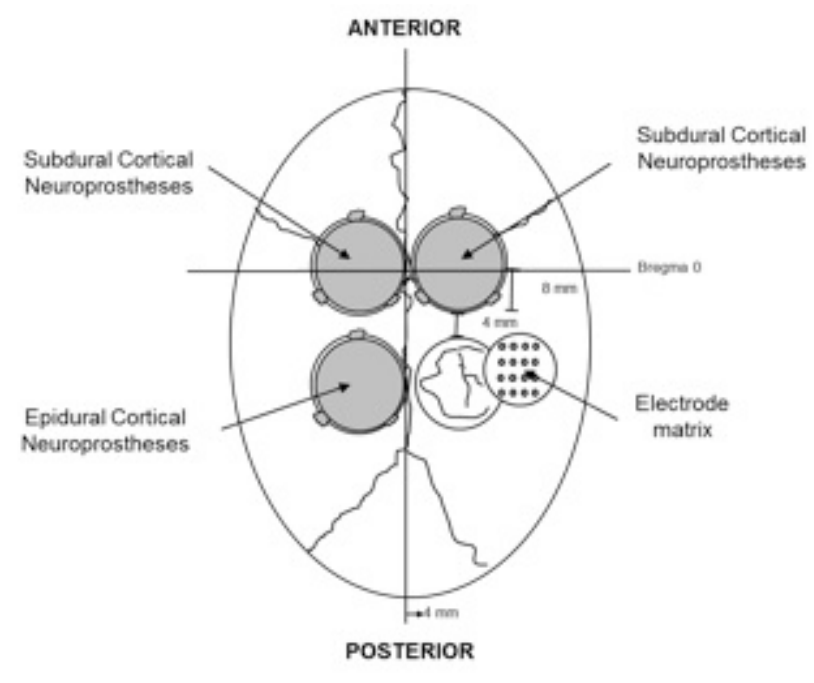


Session Title: Papers Session - Best Science II

Session Time: Wednesday, May 29, 2013, 10:50 am - 12:00 noon

Presentation Number: 77

\section{Nerve Fiber Composition Of Posterior Subthalamic Area And Its Relevance For The Treatment Of Parkinson'S Disease Symptoms. \\ Francisco Velasco, Hospital General de Mexico, Mexico DF, Mexico; Guadalupe García, Ana Luisa Velasco and Luis Concha,}

Introduction: posterior subthalamic area (PSA) electrical stimulation has been proven successful in treating all symptoms of Parkinson's disease (PD). PSA is composed mainly by fibers of unknown origin; the purpose of this study was to determine connectivity of PSA through high resolution MRI-DTI.

Methods: Ten subjects: 5 with unilateral PD and 5 paired controls had 3T MRI, 126 directions studies to be analyzed for DTI of PSA. In each case the boundaries of PSA structures, i.e: Red nucleus (Ru), caudal zona incerta (Zic), subthalamic nucleus (STN) and prelemniscal radiations (Raprl) were delineated in axial sections and seeding performed for each structure to determine their probabilistic connectivity. The five PD patients were thereafter operated to place DBS electrodes in our conventional target in PSA and followed for a period of 1 year to determine the degree of improvement through UPDRSIII scale.

Results: Three main tracts crossed the PSA: brachium conjunctivum cerebellar-thalamic fibers, a caudal extension of the ansa lenticularis from Gpi to PPN and a midbrain tegmental to orbitofrontal cortex tract. DBS electrodes were placed at the intersection of these fibers and induced from 78 to 100\% improvement of tremor, bradykinesia and rigidity. No significant differences were found between affected and non-affected hemispheres in PD or PD and control groups.

Conclusions: Fiber composition of PSA explains the effect of DBS on main PD symptoms: cerebellar-thalamic fibers for rigidity and tremor; Tegmental-orbitofrontal for bradykinesia and Gpi to PPN for gait disturbances 


\section{Modulation of Neural Network Excitability with an Implantable Closed-Loop DBS System}

Paul Stypulkowski, PhD, Medtronic, Minneapolis, MN; Jon Giftakis, PhD, Minneapolis, MN; Scott Stanslaski, Minneapolis, MN; Randy Jensen, Minneapolis, MN; David Carlson, Minneapolis, MN; Pedram Afshar, MD, PhD, Minneapolis, MN; Tim Denison, PhD, Minneapolis, $\mathrm{MN}$

Introduction: In conjunction with therapeutic stimulation, next generation DBS devices may offer advanced features, including the ability to record and analyze neural signals, providing unprecedented insight into DBS effects on neural networks, and the potential to titrate therapy based on physiological observations. We recently reported the first chronic evaluation of an implantable, clinical-grade system that permits concurrent stimulation and recording, in a large animal (ovine) model developed to study DBS for epilepsy (Stypulkowski et al., 2013). Here we describe some of the advanced capabilities of this system, including closed-loop control of neural network excitability.

Methods: Following anesthesia and 1.5T MRI acquisition, unilateral anterior thalamic (Model 3389) and hippocampal (Model 3387) leads were implanted in three animals using a frameless stereotactic system, and connected to modified Activa PC neurostimulators. Chronic, awake recordings of local field potentials (LFPs) and evoked potentials (EPs) were collected with the implanted device and analyzed off-line. For closed-loop function, LFP power level thresholds were set and real-time control of stimulation was implemented through embedded device algorithms.

Results: Consistent with our earlier reports, anterior thalamic DBS or direct stimulation of the hippocampus produced parameterdependent effects on hippocampal activity (Figure 1). Suppression of LFPs, suggesting a reduction in network excitability, could be reliably produced with specific stimulation parameters. This reduced excitability was confirmed by: 1) decreased evoked potential amplitudes, and 2) increased after-discharge thresholds, when measured following pre-conditioning stimulation. Finally, real-time modulation of network excitability via the implanted device was demonstrated using theta-band power level as a control signal for closed-loop stimulation.

Conclusion: These results provide further insight into DBS therapy for epilepsy, and an encouraging demonstration of the capabilities of this new technology, which may afford unique opportunities to study human brain function and neuromodulation mechanism of action in ways that were previously unavailable.

Stimulation Amplitude Effects
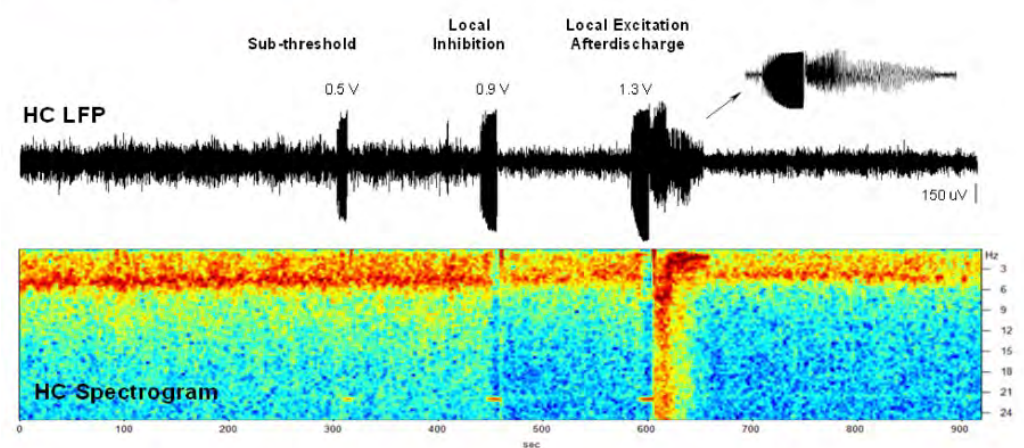

Stimulation Frequency Effects
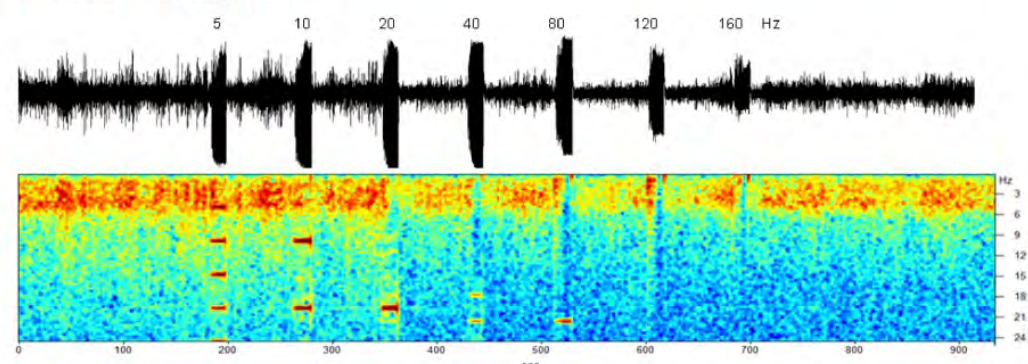


\section{Session Title: Papers Session - From the Laboratory to the Clinic}

Session Time: Wednesday, May 29, 2013, 2:00 pm - 3:00 pm

Presentation Number: 380

\section{In Vivo Brain Repair? Electrical Fields Attract Newborn Brain Cells}

Ali Jahanshahi, Maastricht University, Maastricht, Netherlands; Lisa Schönfeld, Maastricht, Netherlands; Harry Steinbusch, Maastricht, Netherlands; Jacobus van Overbeeke, Maastricht, Netherlands; Yasin Temel, Maastricht, Netherlands

Introduction: Neurogenesis is known to occur at the subventricular zone (SVZ), where the highest numbers of stem/progenitor cells in adult brain are hosted. Following proliferation in the SVZ, newborn cells mainly migrate rostrally towards the olfactory bulb.

Although specific factors influencing the neurogenesis have been identified, tools controlling the direction of migration of newborn cells are not available.

Methods: We applied electrical fields (EFs) to the rat motor cortex. Animals received BrdU injections to examine the neurogenesis related processes and were scored for behavioral parameters. At the end of the experiments, rats were sacrificed, brains were removed and processed for immunohistochemistry.

Results: Results showed a striking increase cell proliferation in the SVZ following cortical delivery of EFs. We also found increased number of BrdU-positive cells in the area below the electrodes. Furthermore, double labeling of cortical BrdU-positive cells with NeuN showed that newborn SVZ cells not only migrate to the cortex, but also differentiate into mature neurons. Finally, based on the fact that subependymal 5-hydroxytryptamine (5-HT, or serotonin) plexus overlaps with the SVZ neurogenic area and the existing knowledge of the effects on 5-HT on neurogenesis, we proposed that enhanced 5-HT in the SVZ could be responsible for the proliferation boost following cortical delivery of EFs. We found clearly enhanced density of the serotonergic fibers in the SVZ and a concurrent increase in neuronal activity in the dorsal raphe nucleus, the brain's main serotonergic nucleus.

Conclusion: We showed that the application of specific EFs can direct migration of newborn brain cells from the SVZ to the area of interest. In addition, our results suggest that this process to be coordinated by enhanced serotonergic input to the SVZ. We propose the possibility of cortical brain repair after epidurally applied EFs based on the existence of electrotaxis of newborn brain cells. 


\section{Session Title: Papers Session - From the Laboratory to the Clinic}

Session Time: Wednesday, May 29, 2013, 2:00 pm - 3:00 pm

Presentation Number: 202

\section{Deep Brain Stimulation Of The Cm-pf In Rats Alleviate Breeding-induced Deficient Prepulse Inhibition}

Mesbah Alam, PhD, Hannover Medical School, Hannover, Germany; Svilen Angelov, Hannover, Germany; Joachim Kurt Krauss, $\mathrm{MD}, \mathrm{PhD}$, Hannover, Germany; Kerstin Schwabe, PhD, Hannover, Germany

Introduction: In Tourette`s syndrome deep brain stimulation (DBS) of the globus pallidus internus (GPi) and the anterior region of the centromedian parafascicular complex (CM-Pf) have been shown to alleviate tics and associated phenomena possibly related to deficient sensorimotor gating. Breeding-induced deficient prepulse inhibition (PPI) of the acoustic startle response (ASR) has been used as an endophenotype for deficient sensorimotor gating. Recently, we showed that DBS of the rat equivalent of the GPi alleviates breeding-induced deficient PPI. We here investigated whether DBS of the rat CM-Pf would improve breeding-induced deficient sensorimotor gating as well.

Methods: Electrodes were stereotactically implanted bilaterally in the CM-Pf of rats with breeding-induced low and high PPI. After two weeks of recovery, rats were stimulated with $100 \mu \mathrm{A}$ and $150 \mu \mathrm{A}$, or sham-stimulated with $0 \mu \mathrm{A}(130 \mathrm{~Hz}$ and $80 \mu$ s pulse width) for epochs of five days via a cable connected to a stimulator with a swivel interposed to allow free movement of the rats. At the end of each epoch the effect of ongoing stimulation on PPI was tested.

Results: CM-Pf DBS alleviated sensorimotor gating in PPI low rats with $150 \mu \mathrm{A}$ being more effective than $100 \mu \mathrm{A}$. In PPI high rats, stimulation had no effect. ASR was not affected by stimulation in PPI high and low rats. Histological analysis verified electrode placement in the CM-Pf.

Conclusions: This work indicates an important role of the CM-Pf in the modulation of sensorimotor gating. This model may be useful to further investigate the pathophysiological mechanisms of deficient sensorimotor gating and mechanisms of action of DBS in certain neuropsychiatric disorders. 


\section{Session Title: Papers Session - From the Laboratory to the Clinic}

Session Time: Wednesday, May 29, 2013, 2:00 pm - 3:00 pm

Presentation Number: 58

Theta Burst Stimulation Of The Hippocampus Via An Electrode Implanted In The Fornix Is Associated With Improved Cognitive Performance After Moderate Traumatic Brain Injury

Jonathan Miller, University Hospitals Case Medical Center/Case Western Reserve University School of Medicine, Cleveland, $\mathrm{OH}$; Katherine Eakin, Cleveland, OH; Jennifer Sweet, MD, Cleveland, $\mathrm{OH}$

Introduction: Learning and memory deficits are a source of considerable morbidity after traumatic brain injury (TBI). We investigated whether stimulation of the hippocampus using theta burst stimulation (TBS) via an electrode implanted in the dorsal fornix would improve performance after moderate TBI in cognitively demanding tasks.

Methods: Male Sprague-Dawley rats (300-350 g) underwent midline fluid percussion over the sagittal sinus (1.9 atm) and were compared to sham-operated rats. In a subset of rats a stimulating electrode was implanted into the dorsal fornix and positioned by production of an evoked potential in the hippocampus. Rats were tested using a 60-second delayed non-match to sample (DNMS) swim T-maze and Morris water maze. TBS (200 Hz in $50 \mathrm{~ms}$ trains, 5 trains per second, $60 \mu \mathrm{A}$ biphasic pulses) was administered during the DNMS swim T-maze task and one hour prior to entry into the water maze. Performance was compared among sham, TBI, and TBI+TBS groups for each task.

Results: Stimulation of the fornix electrode generated a robust evoked potential in the hippocampus that was proportional to stimulation amplitude. In the DNMS swim T-maze, there was a significant difference in performance between TBI and TBI+TBS groups $(p<0.05)$, but no difference between sham and TBI+TBS. In the Morris water maze, latency in TBI+TBS rats was significantly different from TBI starting on day $2(\mathrm{p}<0.05)$ and was not different from sham-injured rats. TBS+TBI rats performed significantly more platform crossings in the probe trial $(\mathrm{p}<0.01)$ and exhibited improved search strategy, with significantly decreased time spent within $10 \mathrm{~cm}$ of the maze wall, starting on day $3(\mathrm{p}<0.05)$.

Conclusions: Deficits in learning and memory after TBI are improved with theta burst stimulation of the hippocampus via an electrode implanted in the dorsal fornix. 
Presentation Number: 317

\section{Recordings of Local Field Potentials in the Bed Nucleus of the Stria Terminalis in a Rat Model of Obsessive-Compulsive Disorder}

Hemmings C. Wu, K.U.Leuven, Leuven, Belgium; Tim Tambuyzer, Leuven, Belgium; Kyra Bauweleers, Leuven, Belgium; Kris van Kuyck, Leuven, Belgium; Jean-Marie Aerts, Leuven, Belgium; Bart Nuttin, Leuven, Belgium

Introduction: In the schedule-induced polydipsia (SIP) model, a recognized rat model of compulsive behavior, rats receive food pellets under a fixed-time schedule each day, and gradually develop compulsive drinking behavior. The objective of our research is to investigate the properties of local field potentials (LFPs) in the bed nucleus of the stria terminalis (BNST) in SIP rat.

Methods: 32 Wistar rats are randomly allocated into three different groups: SIP BSNT (N=12), SIP RND (N=12) and CON BNST $(\mathrm{N}=8)$. Electrodes are implanted in BNST bilaterally in SIP BNST rats, in control groups (CON BNST), and in random targets in rats of SIP RND group.

On the first three days of conditioning every rat from all three different groups is individually placed in a conditioning cage for one hour, in which it receives 60 food pellets at once (CON conditioning). After three days of baseline measurement, rats in SIP BNST and SIP RND undergo SIP conditioning (1 pellet delivered every minute for one hour) while rats in CON BNST continue undergoing CON conditioning. These conditionings continue for 19 consecutive days. Each rat is wired to a data acquisition system to allow LFP recordings during the entire conditioning periods (22 days in total). Water intake during conditioning is documented daily, and a webcam is fixed on top of each conditioning cage to record rat behavior. LFPs and videos are post-processed offline by custom Matlab scripts.

Results: We observe specific behavioral pattern in SIP rat from video analysis. Preliminary power spectral analysis shows that the frequency powers evolve during the development of SIP behaviors. Further analysis to correlate LFP and SIP behavior is in progress. Conclusions: Recording of synchronized LFP and behavior in freely-moving SIP rat is technically feasible. Additional BNST LFP analysis is ongoing to further reveal underlying neurobiological mechanism of SIP. 


\section{Session Title: Papers Session - From the Laboratory to the Clinic}

Session Time: Wednesday, May 29, 2013, 2:00 pm - 3:00 pm

Presentation Number: 217

STN -Deep Brain Stimulation In OCD: Preliminary Results Of The Grenoble Cohort At 18 Months Follow Up stephan Chabardès, MD,PHD, University hospital, GRENOBLE, France; mircea Polosan, MD,PHD, GRENOBLE, France; Paul Krack, MD,PHD, GRENOBLE, France; Brigitte Piallat, PHD, GRENOBLE, France; Alexandre Krainik, MD,PHD, GRENOBLE, France; Eric Seigneuret, MD, GRENOBLE, France; Olivier David, PHD, GRENOBLE, France; Thierry Bougerol, MD,PHD, GRENOBLE, France; Alim L. Benabid, MD,PHD, GRENOBLE, France

OCD are characterized by recurrent unwanted ideas, images or impulses (obsessions) that induce repetitive stereotyped behaviors or mental acts (compulsions). Despite optimal treatment and CBT, 20 to $40 \%$ of patients remain severely disabled. DBS has been proposed as an alternative treatment in resistant forms and recent studies have suggested that non motor loops of basal ganglia were involved in OCD. We report here the results of 14 consecutive OCD patients, treated by bilateral non motor STN-DBS, at 18 months follow-up.

Material and methods. Between 2005 and 2010, 14 patients (5 males and 9 females), were operated on and prospectively followed at the Grenoble university hospital. Mean duration of the disease was 19,07 years and mean pre-op YBOCS was 34 (median 33). Surgery consisted in bilateral implantation of DBS lead in the non motor STN target (laterality 10mm, AP: $2 \mathrm{~mm}$ anterior to motor STN, depth $-4 \mathrm{~mm}$ below AC-PC).

Results: Surgery was safe in all patients but one experienced transient aphasia after left thalamic contusion. One patient was explanted because of behavioral disorders due to preexisting pathological personality. Monopolar stimulation was used in 10/13 at $130 \mathrm{hz}, 60 \mu \mathrm{s}$, 1.2 to 2.4v. Chronic STN high frequency stimulation induced hypomanic symptoms in 2 patients and anxiety in 2 patients that was reversible after fine tuning of stimulations parameters. Twelve out of 13 had a YBOCS improvement of more than $35 \%$ and 7 had more than $50 \%$. Mean post-op improvement was $58.9 \%$ (median $48.4 \%$ ).

Conclusion: Non-motor STN-DBS in severe OCD patients was safe and could result in 7/13 cases in an improvement greater than 50 $\%$. Side effects were transient in most of cases or reversible after fine tuning of stimulation parameters. Selection criteria and predictive factors of improvement will have to be refined in larger studies 
Presentation Number: 289

Motor Cortex Stimulation Efficacy In Refractory Neuropathic Pain Might Be Predicted By Brain ${ }^{11}$ C-diprenorphine Pet-scans Patrick MERTENS, MD, PhD, University LYON 1, LYON, France; Joseph Maarrawi, MD,PhD, Beyrouth, Lebanon; Roland Peyron, MD, PhD, Saint Etienne, France; Luis Garcia Larrea, MD, PhD, LYON, France

Introduction: Clinical effect of motor cortex stimulation (MCS) for neuropathic pain (NP) relief has been suggested to be mediated by secretion of endogenous opioids, both in humans and animal models. Since NP itself is associated with changes in the brain opioid system, we investigated whether the magnitude and distribution of opioid receptors in NP patients could become a biological marker of the ability of MCS to control their pain.

Methods: Opioid receptor availability was assessed using ${ }^{11} \mathrm{C}$-Diprenorphine PET-scans in 15 patients suffering refractory NP, who were subsequently treated by implantation of MCS clinically assessed after 7 months of chronic MCS. Eleven healthy subjects of age and sex distribution matched with those of patients benefited also from 1 session of ${ }^{11} \mathrm{C}$-Diprenorphine PET-scans.

Results: Level of preoperative opioid-binding in insula, thalamus, periaqueductal grey, anterior and middle cingulate, orbito-frontal cortices, and amygdalae was significantly correlated with postoperative pain relief at 7 months. NP patients with mean BP (Binding potential) values lower than the BP range of normal age-matched controls in the thalamus, PAG, and contralateral insula are those who are less likely to benefit from MCS.

Conclusions: Pre-operative ${ }^{11} \mathrm{C}$-Diprenorphine PET-scans might be used in the future as a predictive tool of MCS efficacy and help clinicians to select the best candidates to MCS. 
Presentation Number: 67

\section{SimultaneousTrial of Deep Brain Stimulation (DBS) and Motor Cortex Stimulation (MCS) for Chronic, Intractable Neuropathic Pain}

Byung-chul Son, MD, PhD, St. Vincent's Hospital, The Catholic University of Korea, Suwon, Kyeonggi-do, Korea, Republic of; Hyung-suk Kim, MD, Suwon, Kyeonggi-do, Korea, Republic of; Deog-ryeong Kim, MD, Suwon, Kyeonggi-do, Korea, Republic of; Sang-Won Lee, MD, PhD, Suwon, Kyeonggi-do, Korea, Republic of

Introduction: Both Motor cortex stimulation (MCS) and Deep brain stimulation (DBS) of thalamus have been shown to be effective in both central and peripheral neuropathic pain and shares common pathophysiologic mechanism of analgesia; modulation of thalamic activity. However, there is still no concensus when we try DBS and MCS for this chronic pain and which one is better. We performed trial of DBS and MCS simultaneously in 9 patients with chronic neuropathic pain and report long-term outcome of simultaneous trail of DBS and MCS.

Methods: Among 25 patients with thalamic DBS and 18 patients with MCS for various kinds of chronic neuropathic pain, simultaneous trial of DBS and MCS were performed in 9 patients. The mean duration of follow-up was 39 months (range, 72-8 months). Their causes of pain were; CPSP ( $\mathrm{n}=4)$, SCI pain ( $\mathrm{n}=4)$, and stump and neuroma pain in the arm.

DBS of sensory thalamus was performed first in local anesthesia and general anesthesia was conducted for MCS. Both the distal ends of the electrodes for DBS, MCS were externalized for postoperative trial stimulation.

Results: After trial stimulation, 6 MCS and 2 DBS were successful, and permanent stimulation was given. During the follow-up (average, 39 months, range 8-72 months), the average pain reduction in MCS-treated patient was $41.58 \%$ ( $\mathrm{n}=5$, range, 28.1-57.1\%, preop. NRS. 7.4, last follow-up NRS 4.5) and 50\% in DBS-treated group ( $\mathrm{n}=2$, preop. NRS 8, follow-up NRS 4). There was no difference in degree of pain relief between DBS and MCS-treated group ( $\mathrm{p}>0.005)$.

Conclusions: Our study showed that the success rate of MCS was higher than DBS in chronic neuropathic pain and MCS was more effective in patients with CPSP. However, the degree of analgesic efficacy was not different between DBS and MCS-treated groups. 
Presentation Number: 234

\section{Differential Efficacy Of Motor Cortex Stimulation And Lesioning Of The Dorsal Root Entry Zone For Continuous Vs Paroxysmal Pain Afterroot Avulsion}

Youichi Saitoh, Osaka University Graduate School of Medicine, Suita, Osaka, Japan; Satoru Oshino, MD, PhD, Suita, Osaka, Japan; Koichi Hosomi, MD, PhD, Suita, Osaka, Japan; Haruhiko Kishima, MD, PhD, Suita, Osaka, Japan; Tomoyuki Maruo, MD, PhD, Suita, Osaka, Japan; Toshiki Yoshimine, MD, PhD, Suita, Osaka, Japan

Introduction: Pain after root avulsion (RA) has 2 distinct patterns: continuous burning pain and paroxysmal shooting pain. Lesioning of the dorsal root entry zone (DREZotomy) is more effective for paroxysmal than continuous pain. It is unknown, however, whether electric motor cortex stimulation (EMCS) has a differential effect on continuous vs paroxysmal BPA pain. To analyze the differential effect of EMCS and DREZotomy on continuous vs paroxysmal BPA pain in a series of 15 patients.

Methods: Fifteen patients with intractable RA pain underwent DREZotomy alone $(\mathrm{n}=7)$, EMCS alone $(\mathrm{n}=4)$, or both procedures (n =4). Pain intensity was evaluated with the Visual Analog Scale, and separate ratings were recorded for paroxysmal and continuous pain. Pain relief was categorized as excellent ( $>75 \%$ pain relief $)$, good $(50 \%-75 \%)$, or poor $(<50 \%)$. Favorable outcome was defined as good or better pain relief.

Results: Eight patients had EMCS; 7 were followed up for an average of 47 months. Of those 7 patients, 3 (42\%) with continuous pain had favorable outcomes compared with no patients with paroxysmal pain. Eleven patients had DREZotomy; 10 were followed up for an average of 31 months. Of those 10 patients, 7 (70\%) with paroxysmal pain had

favorable outcomes compared with $2(20 \%)$ with continuous pain.

Conclusions: EMCS was ineffective for paroxysmal pain but moderately effective for continuous pain. DREZotomy was highly effective for paroxysmal pain but moderately effective for continuous pain. It may be prudent to use EMCS for residual continuous pain after DREZotomy.

In addition, we will discuss about non-invassive rTMS efficacy for RA pain.

\section{DREZotomy}

Long-term follow-up; mean 31 months

\section{EMCS}

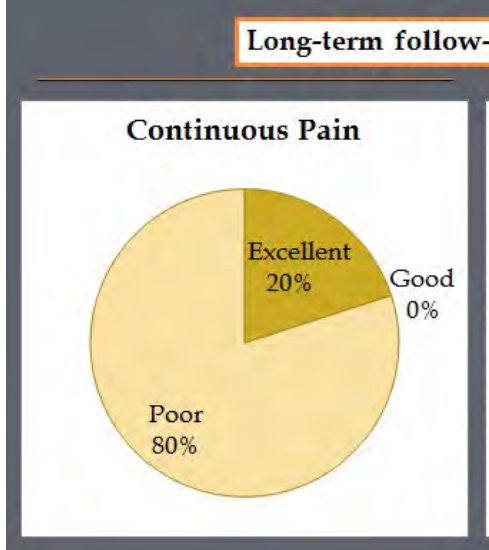

Long-term follow-up; mean 47 months

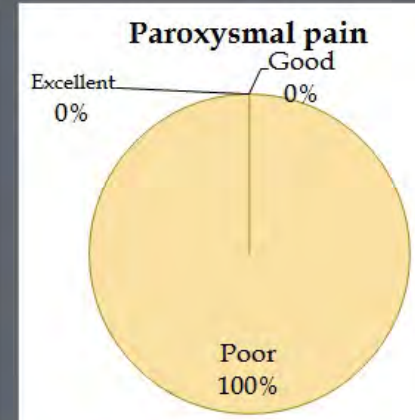

Continuous pain

$100 \%$

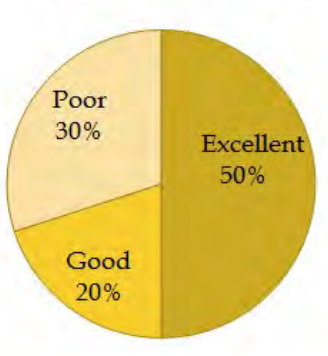

Paroxysmal pain 
Presentation Number: 211

Deep Brain Stimulation In Chronic Cluster Headache (CCH): Lead Location, Clinical Response And Neuronal Signatures Fahid T. Rasul, MRCS, MSc, National Hospital for Neurology and Neurosurgery, London, United Kingdom; Susie Lagrata, London, United Kingdom; Alek Pogosyan, Oxford, United Kingdom; Peter Brown, Oxford, United Kingdom; Marwan Hariz, London, United Kingdom; Manjit Matharu, London, United Kingdom; Ludvic Zrinzo, London, United Kingdom

Introduction: $\mathrm{CCH}$ is refractory to medical therapy in a small minority of patients. Neuroimaging studies implicate the ipsilateral posterior hypothalamic region (PH) in its pathogenesis. PH deep brain stimulation (DBS) has shown promise in the management of refractory CCH. Here we investigate the value of local field potentials (LFPs) relative to the site of active DBS contact used for chronic stimulation.

Methods: Four patients with refractory unilateral CCH treated with MRI-guided and MRI-verified approach DBS of the PH without microrecording were investigated. The target on stereotactic T2-weighted images lay between the anteromedial quadrant of the red nucleus and the mammillary bodies. LFPs were recorded from externalised wires prior to implantation of the impulse generator a week after lead implantation.

Results: All leads were within 1.0mm of the intended target point. Mean(range) coordinates of the chronically active contact were 3.6(2.0-5.40)mm lateral, 3.1(1.2-5.0)mm posterior and 5.0(2.0-8.6) $\mathrm{mm}$ inferior to the midcommissural point. Median follow-up was 25(15-26)months. Preoperatively, median headache load was 397(270-751). Headache load was defined as the [severity (on the visual analogue scale)] x [duration] x [frequency] of cluster headache attacks. Median headache load fell to 22(0-280) per month at latest follow-up, a reduction of 57, 58, 68 and $100 \%$ in each patient. Stimulation was monopolar, with $1-3 \mathrm{~V}$ at $185 \mathrm{~Hz}$ and $60 \mu$ s throughout the follow-up period. No sustained side effects related to stimulation were reported.

LFP peaks were noted in 3 out of 4 cases (at 8,11 and 15Hz). The contact(s) used for stimulation at last follow-up coincided with one or both of the contacts affording the highest amplitude peak in bipolar recordings.

Conclusions: Our clinical results support previous published data suggesting that PH-DBS may be useful in patients with medically refractory cluster headache. LFP activity may potentially be useful in guiding contact selection during programming and deserves further investigation. 
Presentation Number: 144

\section{Rechargeable Occipital Nerve Stimulator Systems a Patient Satisfaction Study}

Sara Sciacca, National Hospital for Neurology and Neurosurgery, London, United Kingdom; James Shand Smith, London, United Kingdom; Harith Akram, London, United Kingdom; Aymen Asim, London, United Kingdom; Manjit Matharu, London, United Kingdom; Laurence Watkins, London, United Kingdom

Introduction: Cluster headache is a disabling severe headache syndrome, often termed as "suicide headache", with a prevalence of $0.12 \%$. Occipital nerve stimulation (ONS) is a recognised treatment for medically intractable cluster headache. The system is composed of two electrodes implanted under the occipital scalp in contact with the greater occipital nerves and connected, through leads tunnelled under the skin to, an Implantable Pulse Generator (IPG) and battery. This is implanted in a fat pocket in the subclavial area or the abdomen to provide electrical stimulation. The battery can be non-rechargeable or rechargeable, which has a longer lifespan. This is, to our knowledge, the largest patient survey to look at the level of satisfaction with rechargeable batteries.

Methods: Using the ONS database at the National Hospital for Neurology and Neurosurgery, Queen Square, London we identified 100 patients who have had rechargeable systems implanted between January 2006 and September 2012. We used a patient satisfaction questionnaire which we developed with some of the questions adapted from Mcauley et al. regarding rechargeable IPGs for spinal cord stimulation. We gathered the data via a telephone interview, verbal consent was obtained from the patients.

Results: 92 out of the 100 patients consented to participate. 68 patients (74\%) found recharging the battery convenient and $81(88 \%)$ found that the amount of inconvenience associated with recharging worth the benefit they receive from the stimulation while $3(3 \%)$ did not. Of the 92 patients 48 (53\%) had a non-rechargeable battery before the current rechargeable one, and 37 of them (84\%) stated preferring the current rechargeable to the previous non-rechargeable.

Conclusions: The results indicate a positive general outcome of ONS with rechargeable battery. The data suggest that ONS with rechargeable battery should be offered as first option to patients undergoing ONS implant. 
Presentation Number: 343

\section{Steering Deep Brain stimulation: an exploratory study with a new 32-contact lead}

Rick Schuurman, MD, PhD, Academic Medical Center Amsterdam, Amsterdam, Netherlands; Fiorella Contarino, MD, PhD, Amsterdam, Netherlands; Lo Bour, PhD, Amsterdam, Netherlands; Rob de Bie, MD, PhD, Amsterdam, Netherlands; Pepijn van den Munckhof, Md, PhD, Amsterdam, Netherlands

Introduction: Deep brain stimulation (DBS) performed through the currently used leads with 4 circular contact points may cause side effects, along with the clinical effect. This is partly due to the unwanted stimulation of adjacent structures. A system capable of steering stimulation could reduce and raise the threshold for the occurrence of side effects. Objectives of this study are 1) to confirm that stimulation through a new 32-contact lead can produce equivalent effects as stimulation through currently available commercial DBS leads, and 2) to establish that steering stimulation with successive segmental activations in a 32-contact lead modulates the threshold for stimulation-induced effects.

Methods: Ten patients with Parkinson's disease are included in the study. The new 32-contact electrode is temporarily inserted during surgery and double blind intraoperative test will be performed to evaluate the threshold for benefit and the threshold for side effects in a ring mode and in 4 different steering directions. Results will be compared with those obtained from stimulation with a conventional electrode.

Results: The study is a single-center, single-group, performance and safety study, conducted during DBS surgery, of which the results will be presented.

Conclusions: The new 32-contact lead employs a larger number of smaller electrodes. This allows stimulation to be steered selectively towards targeted areas, thus sparing other structures. In this way the therapeutic window for stimulation could be improved. 
Session Title: Papers Session - Stereotactic Technology

Session Time: Wednesday, May 29, 2013, 2:00 pm - 3:00 pm

\section{Presentation Number: 412}

\section{Convection Enhanced Delivery: Infusion characteristics in ex vivo and in vivo non-human primate brain tissue}

Gurwattan S. Miranpuri, PhD, University of Wisconsin, Madison, WI; Angelica Hinchman, Madison, WI; Anyi Wang, Madison, WI; Ken Kubota, Los Altos, CA; Martin Brady, Baltimore, MD; Raghu Raghavan, Baltimore, MD; Kevin Bruner, Madison, WI; Ethan Brodsky, Madison, WI; Walter Block, Madison, WI; Ben Grabow, Madison, WI; Andrew Alexander, Madison, WI; Jim Raschke, Los Altos, CA; Chris Ross, Pembroke Pines, FL; Heather Simmons, Madison, WI; Karl Sillay, Madison, WI

Introduction: Convection enhanced delivery (CED) is a new method in treating Parkinson's Disease. The current study compares $\mathrm{Vd} / \mathrm{Vi}$ ratios and backflow measurements following CED infusions in ex vivo versus in vivo non-human primate brain tissue, based on infusion protocols developed in vitro.

Methods: In ex vivo infusions, the first brain received 2 infusions using a balloon catheter at rates of $1 \mu \mathrm{L} / \mathrm{min}$ and $2 \mu \mathrm{L} / \mathrm{min}$ for 30 minutes. The second and third brains received infusions using a valve-tip (VT) catheter at $1 \mu \mathrm{L} / \mathrm{min}$ for 30 minutes. The fourth brain received a total of $45 \mu \mathrm{L}$ infused at a rate of $1 \mu \mathrm{L} / \mathrm{min}$ for 15 minutes followed by $2 \mu \mathrm{L} / \mathrm{min}$ for 15 minutes. Imaging was performed (SPGR FA34) every 3 minutes. In in vivo, 4 subjects received a total of 8 infusions of $50 \mu \mathrm{L}$. Subjects 1 and 2 received infusions at $1.0 \mu \mathrm{L} / \mathrm{min}$ using a VT catheter in the left hemisphere and a smart-flow (SF) catheter in the right hemisphere. Subjects 3 and 4 each received 1 infusion in the left and right hemisphere at $1.0 \mu \mathrm{L} / \mathrm{min}$.

Results: Backflow and infusion morphology measurements were compared with the Vd/Vi ratios of the ex vivo and in vivo trials with 1) iterative MRI and 2) pathology slice measurements to calculate the $\mathrm{Vd} / \mathrm{Vi}$. MRI calculations of $\mathrm{Vd} / \mathrm{Vi}$ did not significantly differ from those obtained on post-mortem pathology. The mean measured Vd/Vi of in vivo (5.23+/-1.67) compared to ex vivo (2.17+/-1.39) demonstrated a significantly larger $\mathrm{Vd} / \mathrm{Vi}$ for in vivo by 2.4 times $(\mathrm{p}=0.0017)$.

Conclusions: We detected higher ratios in in vivo subjects than in ex vivo. This difference could be explained by the extra cellular space volume fraction. Studies evaluating backflow and morphology use in vivo tissue as a medium are recommended. Further investigation is warranted to evaluate the role blood pressure and heart rate may play in human CED clinical trials. 


\section{Accuracy of Electrode Placement, Clinical Response and Complication rate in Frameless Stereotaxis (NexFrame) for Movement disorders}

Matthew G. Stovell, MBBS BSc MRCS, The Walton Centre for Neurology \& Neurosurgery, Liverpool, United Kingdom; Emman J. Qattan, BSc MSc, Liverpool, United Kingdom; Patricia Byrne, BSc RGN, Liverpool, United Kingdom; Naeem A. Farooqi, MBBS FRCS (Neurosurgery), Liverpool, United Kingdom; Petr Janous, MD, Liverpool, United Kingdom; Paul R. Eldridge, FRCS (Neurosurgery), Liverpool, United Kingdom; Jibril Osman Farah, FRCS (Neurosurgery), Liverpool, United Kingdom

Introduction: The NexFrame is a frameless stereotactic image guided system used to perform surgery for deep brain stimulation. We present a retrospective analysis of our experience with 108 patients (201 leads implanted) with particular emphasis on accuracy, clinical response and complication rate.

Methods: From 2007-2012, 108 patients were implanted (age range 24-75) for a variety of indications. All patients underwent $\mathrm{MRI} / \mathrm{CT}$ under GA: CT was used for registration; targeting was MRI based with atlas assistance. MER was used in all cases with a single trajectory. Intra-operative macrostimulation was performed to assess clinical effectiveness and side effects. Immediate postoperative CT scans were acquired and fused to the planning MRI to assess accuracy and identify complications.

Results: 108 patients' data were available for analysis (201 leads). $48 \%$ of patients had excellent clinical response, $49 \%$ had goodmoderate clinical response, $1 \%$ had no change and $2 \%$ had progressive deterioration of the original clinical condition.

Accuracy analysis was possible in 103 leads; the error was $2.51 \mathrm{~mm}$ (S.D. 1.59) when MER was concordant with anatomical targeting and $2.80 \mathrm{~mm}$ (S.D. 1.52) when target was adjusted intra-operatively. Standard deviation in the $\mathrm{x}, \mathrm{y}$ and $\mathrm{z}$ direction were calculated.

We experienced $1.7 \%$ primary infection rate and $2.5 \%$ delayed infection rate. 5 leads were revised for hardware failure, 2 leads were revised for technical error and 5 for delayed clinical response. There were no mortalities and one case of delayed ICH in our series.

Conclusions: The Nexframe frameless system offers satisfactory accuracy with correspondent good clinical response in a wide range of clinical indications. The procedural complication rate was negligible. Improvement of the system may be necessary for increased ease of use, or implantation of leads with iMRI. 
Session Title: Papers Session - Stereotactic Technology

Session Time: Wednesday, May 29, 2013, 2:00 pm - 3:00 pm

Presentation Number: 319

\section{Improved Intraoperative Therapeutic Window with Directional DBS Compared to Omnidirectional DBS Using a Novel Lead Design}

Claudio Pollo, MD, University Hopsital, Bern, Switzerland; Lennart Stieglitz, MD, Bern, Switzerland; Markus Oertel, MD, Bern, Switzerland; Alain Kaelin-Lang, MD, PhD, Bern, Switzerland; Michael Schüpbach, MD, PhD, Bern, Switzerland

Introduction: Deep brain stimulation (DBS) is currently carried in all directions around the stimulation lead. Computational models show that directional leads may improve focalization of current flow in the brain but their clinical application has not yet been investigated. We report four cases of an on-going intraoperative study using directional DBS.

Methods: Three males with Parkinson's Disease underwent STN DBS and one male with Essential Tremor underwent Vim DBS, including intraoperative microrecording and macrostimulation. At the target determined for the permanent electrode, directional stimulation was assessed using a novel lead (directSTIM ${ }^{\mathrm{TM}}$, Aleva Neurotherapeutics, Switzerland). It features two rings of three independent electrodes of $1 \mathrm{~mm}^{2}$ (figure). The angular position of the electrodes allows stimulation at $0^{\circ}, 120^{\circ}$ and $240^{\circ}$ directions. The parameters of stimulation were monopolar, $90 \mu \mathrm{s}$, and $130 \mathrm{~Hz}$. The therapeutic window (TW) is defined as the electrical current threshold at which side effects occur minus the current threshold at which a significant therapeutic effect on motor symptoms is observed (low boundary=TWLB). The TW was measured in each direction (TWdir) and the best TWdir was compared to omnidirectional stimulation (TWomni).

Results: $2 / 4$ patients showed a ratio TWdir/TWomni $\leq 0.25 .1 / 4$ did not show a ratio $<1$. The ration could not be measured on $1 / 4$ patient. The best directional TWLB was $0.63 \mathrm{~mA}(0.3-0.9 \mathrm{~mA})$ and was lower than the omnidirectional TWLB $1.13 \mathrm{~mA}(0.6-1.95 \mathrm{~mA})$. No added benefit was observed using two electrodes either one above the other or one next to the other.

Conclusions: These first observations suggest that directional TW is increased compared to omnidirectional TW. Moreover, TWLB for therapeutic effect is significantly lower $(<1 \mathrm{~mA})$ in the best direction compared to omnidirectional stimulation.. Therefore, directional stimulation may increase effectiveness of DBS. Further intraoperative and chronic studies are needed to confirm these results.

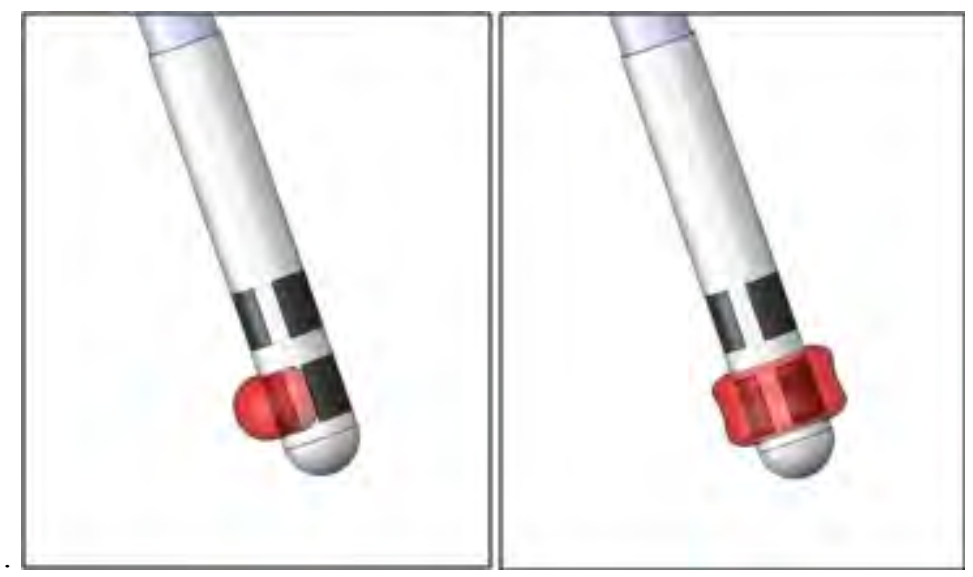

Figure: computed volume of tissue activated with one contact stimulated(left) compared to 3 contacts(right). 
Session Title: Papers Session - Stereotactic Technology

Session Time: Wednesday, May 29, 2013, 2:00 pm - 3:00 pm

\section{Presentation Number: 32}

\section{Techniques For Axiem ${ }^{\mathrm{TM}}$ Electro-magnetic Navigation Guided Balloon Compression Of Trigeminal Nerve}

Sarah L. Olson, FRACS, Princess alexandra hospital, Brisbane, Australia; Brendan Davis, Brisbane, Australia; Joseph GarciaRedmond, Brisbane, Australia; Mary-Anne Xia, Brisbane, Australia; Amelia Granger, Brisbane, Australia; Paul Nichols, Brisbane, Australia

Introduction: Cannulating the foramen ovale under II imaging alone can be cumbersome and difficult in some cases. Whilst there is learning curve with AxiEM after 43 cases the final 10 patients took a mean of 17.3 minutes from induction to transfer to recovery. No patients had their case aborted due to inability to access the foramen

Methods: 43 patients had A CT stealth acquisition prior to surgery. Prior to induction the surgeon prepared a $4 \mathrm{fr}$ catheter $0.75 \mathrm{ml}$ balloon, 14guage $11.4 \mathrm{~cm}$ trucut needle, half strength omnipaque and AxiEMTM navigation.

The AxiEM TM was marked with the steristrips to the length of the trucut biopsy needle as was the balloon catheter with the balloon extended from the tip. The catheter was then attached to a $1 \mathrm{ml}$ syringe filled with $0.75 \mathrm{ml}$ of half strength omnipaque.

The patient underwent induction of anaesthesia.

Hartls landmarks were marked.

Registration was undertaken and a target placed at the foramen ovale.

An entry point was placed in the standard position on the cheek

Under guidance views the AxiEM ${ }^{\mathrm{TM}}$ and trucut needle were inserted into the foramen ovale.

The II was then introduced for a lateral projection and the balloon insufflated for one minute in a new case and three minutes in a case that had had a MVD or percutaenous procedure prior.

Picture 1: Patient prepared for surgery

Picture 2 AxiEM $^{\mathrm{TM}}$ guidance views

Results: The average time from induction to extubation in the first 10 patients was 83 minutes. In the final 10 patients the average time was 17.3 minutes. No patient was aborted do to inabililty to access the foramen ovale

With the exception of one patient who required a pacemaker postoperatively all patients were discharged day one. There were no complications

Conclusion: AxiEM ${ }^{\mathrm{TM}}$ guidance is an effective and efficient way of cannulating the foramen ovale for trigeminal neuralgia
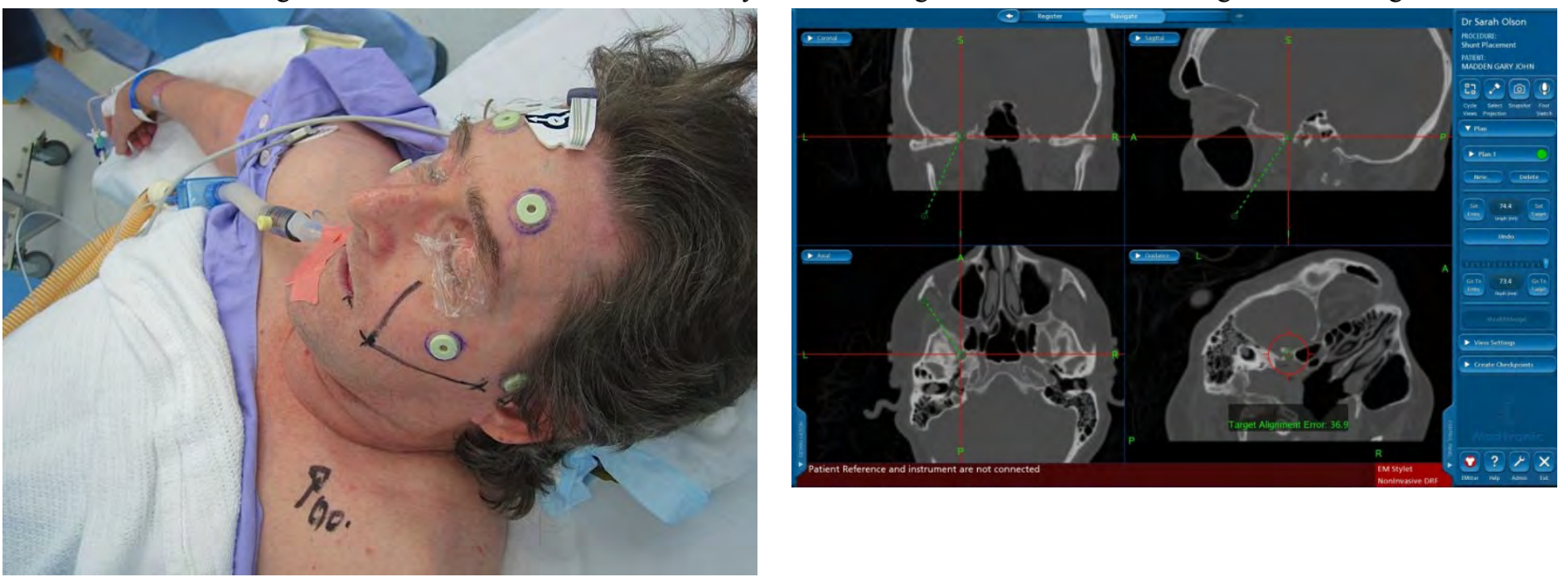
Presentation Number: 377

\section{Influence on Synaptic Plasticity in Corticostriatal Pathway of Parkinson Disease Animal Model}

Yuan-Hao Chen, MD,PhD, Tri-Service General Hospital, Taipei City, Taiwan

Introduction: Using DBS to treat Parkinson's Disease is well documented, but long term effect if synaptic To determine the consequences of dopamine denervation of the striatum on synaptic plasticity and prevention of these changes with gene therapy using an adeno-associated viral vector (AAV) expressing glial cell line-derived neurotrophic factor (GDNF).:

Methods: C57BL6/J mice were injected with the neurotoxin 1-methyl-4-phenyl-1,2,3,6-tetrahydropyridine (MPTP);The synaptic plasticity (ex. long-term depression (LTD) or potentiation (LTP)) of cortico-striatal pathway were measured in vitro. Fast-scan cyclic voltammetry measured electrically released dopamine from a functionally relevant pool in these same striatal slices.

Results: After MPTP, dopamine release and uptake were greatly diminished, and LTP and LTD were blocked in the striatal slices. The loss of plasticity resulted directly from the loss of dopamine since its application rescued synaptic plasticity. Striatal GDNF expression via AAV, before MPTP, significantly protected against the loss of dopamine and prevented the blockade of cortico-striatal LTP.

Conclusions: These data demonstrate that dopamine plays a role in supporting several forms of striatal plasticity and that GDNF expression via AAV prevents the loss of dopamine and striatal plasticity caused by MPTP. We propose that impairment of striatal plasticity after dopamine denervation plays a role in the symptomology of Parkinson's disease and that AAV expression of neurotrophic factors represents a tenable approach to protecting against or slowing these neurobiological deficits. 


\section{Session Title: Flash Session - From the Laboratory to the Clinic}

Session Time: Wednesday, May 29, 2013, 3:30 pm - 4:30 pm

Presentation Number: 284

\section{Autologous Peripheral Nerve Grafts into the SN of Subjects with PD Undergoing DBS}

Craig G. van Horne, MD,PhD, University of Kentucky, Lexington, KY; John Selvin, MD, Lexington, KY; Fariha Zaheer, MD, Lexington, KY; George Quintero, PhD, Lexington, KY; Julie Gurwell, PAc,PhD, Lexington, KY; Renee Wagner, RN, Lexington, KY; Greg Gerhardt, PhD, Lexington, KY

Introduction: PD results from the progressive degeneration of dopaminergic neurons within the substantia nigra pars compacta $(\mathrm{SNpc})$. No current treatment halts or reverses the ongoing degeneration or loss of cell function. This pilot study is designed to test the safety and feasibility of the implantation of autologous peripheral nerve grafts into the SNpc of participants with PD undergoing deep brain stimulation (DBS) surgery. Peripheral nerve tissue contains Schwann cells, which produce growth factors that have been demonstrated to support the survival and function of dopaminergic neurons.

Methods: The study is approved for 6-8 Participants who will be selected from patients with PD who have elected to undergo a standard DBS surgery and treatment for the control of medically refractory symptoms. Participants will serve as their own donor for the tissue. A $5 \mathrm{~mm}$ segment of sural nerve, $1 \mathrm{~mm}$ in diameter, will be harvested and implanted unilaterally into the SNpc through a transplantation cannula at the time of DBS surgery. The primary objective of the study is to evaluate the safety of the implantation procedure in the perioperative period and through the 12 month follow up period. Participants will be evaluated for possible clinical benefits of the graft by measuring motor performance scores (on and off stimulation and medication), and by monitoring changes in therapeutic requirements throughout the follow up period. Neuropsychological evaluations will be performed pre-operatively and at 12 months.

Results: Updated progress data will be presented at the time of the meeting.

Conclusions: The approach of grafting autologous, unaltered, peripheral nerve tissue at the time of DBS surgery is novel and investigates a potentially safe, cost effective treatment for PD that allows participants to receive an approved symptomatic treatment as well as an intervention that could halt or begin to reverse the neurodegenerative process. 


\section{Presentation Number: 184}

\section{Cortical Electric Stimulation in the Treatment Brain Tumors: Induction of Plastic Changes to Obtain Extended Resection in Gliomas}

Juan Barcia, PhD, Madrid, Spain; Josue Avecillas, MD, Hospital Clinico San carlos, Madrid, Spain; Ana Sanz, Madrid, Spain; Juan Raul Brin, MD, Madrid, Spain; Pedro Alonso, MD, Madrid, Spain; Javier Saceda, Madrid, Spain; Miguel Yus, Madrid, Spain; Manuela Jorquera, Madrid, Spain; Mercedes Gonzalez, Madrid, Spain; Maria Victoria Acedo, Madrid, Spain; Tomas Ortiz, Madrid, Spain; Antonio Oliviero, Madrid, Spain

Introduction: Complete resection of gliomas may be impeded by the presence of functional areas within them. As slow tumor invasion promotes topographic plasticity allowing staged tumor resection, as recently described, we aimed to produce a "virtual lesion" in eloquent areas located within tumors either using repetitive transcranial magnetic stimulation (rTMS) or continuous highfrequency cortical electrical stimulation (HFCS), in order to promote plasticity and increase the extent of tumor resection.

Methods: In 4 cases of gliomas invading Broca's or Wernicke's area (1 astrocytoma, 1 oligodendroglioma, 2 anaplastic astrocytoma), previously partially removed using awake cortical monitoring, we stimulated with either rTMS (1 patient), or HFCS ( 3 patients) through an implanted subdural grid, over the eloquent cortex located within the tumor remnant, associated to logopedy. Stimulus intensity was set daily to the threshold of mild speech disturbance

Results: rTMS produced a reinforcement of fluent speech tasks, but not topographic plasticity. On the contrary, HFCS achieved the displacement of speech functions, and a more radical resection was possible in a repeated surgery. Reorganization of language areas was demonstrated with functional magnetic resonance and cortical stimulation. Compromised language areas were identified at the right hemisphere where previously they were absent, or at different places at the left hemisphere than previously found Conclusions: We provide evidence of induced topographic plasticity using HFCS in eloquent areas within a tumor, allowing an increased tumor removal. These results have important implications in brain tumor surgery, rehabilitation and reparative neuroscience. Topographic plasticity can be induced by continuous HFCS and allows enhanced tumor resections.

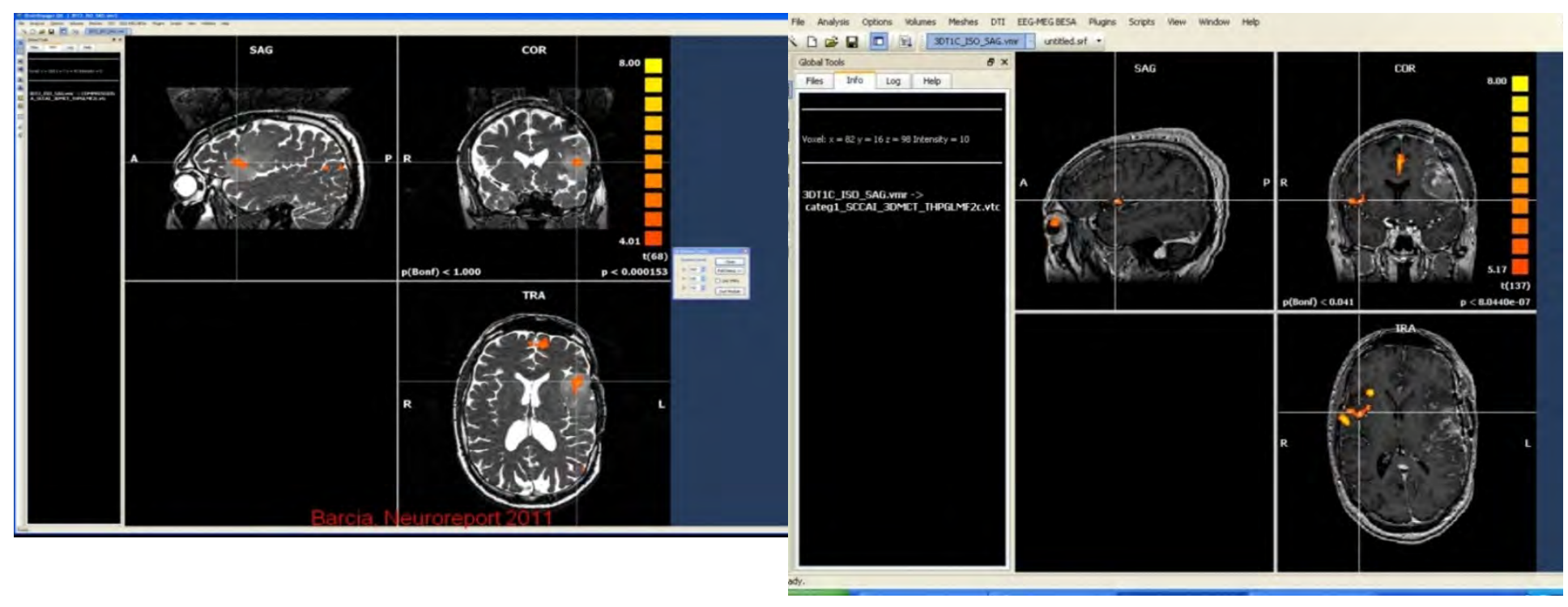




\section{Presentation Number: 44}

\section{Bilateral Pedunculopontine Nucleus Stimulation For Progressive Supranuclear Palsy}

Paresh K. Doshi, MCh (Neurosurgery), Jaslok Hospital and Research Centre, Mumbai, India

Introduction: Pedunculopontine nucleus (PPN) is emerging as a potential target for gait and balance disorders. Targeting PPN can be a challenge, especially in atrophic midbrain of patients with progressive supranuclear palsy (PSP). We report four cases of bilateral PPN stimulation in PSP patients with a short term and long term follow up.

Method: PSP patients with gait disturbances, but were able to walk with or without assistance were selected for this study. The study was approved by the Jaslok Hospital scientific committee. There were three females and one male with average age of 64 years and disease duration of 3 years. The PPN region was targeted using the Proton density images obtained on a 3T MRI, which was fused with stereotactic CT on the day of surgery. DBS electrodes were implanted bilaterally simultaneously without any microelectrode recordings Postoperative programming was performed using low frequency at frequent intervals.

Results: PSP rating scores, subsets 25 (arising from chair), 26 (gait), 27 (postural stability), 28 (sitting down) of PSP rating scale, Motor and total UPDRS were evaluated in ON condition before and at 6 months and 18 months after surgery. The average preoperative scores were, 30, 2, 2.5, 3 and 2 respectively. The mean postoperative scores were 34, 2, 2.5, 1.75 and 3 at six months and $43,3,3,3.6$ and 3 respectively. The total UPDRS was 44, 45 and 65 preoperatively, six months and 18 months respectively; similarly the motor UPDRS was 22, 27 and 35 . There was morbidity or mortality in this series.

Conclusion: Bilateral PPN DBS can be performed in PSP patients. It offers a modest improvement in balance and gait which is sustained at 6 months but continues to deteriorate over time. As compared to the natural progression of the symptom progression is slower in post DBS patient. 


\section{Presentation Number: 47}

\section{Potential Role Of L-PGDS As A Surrogate Marker Of Frontal Lobe Dysfunction In NPH}

Namiko Nishida, MD, PhD, Tazuke Kofukai Kitano Hospital, Osaka, Japan; Nanae Nagata, PhD, Osaka, Japan; Hiroki Toda, MD, $\mathrm{PhD}$, Osaka, Japan; Masatsune Ishikawa, MD, PhD, Kyoto, Japan; Yoshihiro Urade, PhD, Osaka, Japan; Kohichi Iwasaki, MD, PhD, Osaka, Japan

Introduction: Normal pressure hydrocephalus (NPH) is a cause of treatable dementia, gait disturbance, and urinary incontinence in elderly, yet some part of its etiology is still unknown (ie, idiopathic NPH). Lipocalin-type prostaglandin D synthase (L-PGDS) is a major cerebrospinal fluid (CSF) protein produced mainly by arachnoid cells. L-PGDS levels in CSF of NPH patients were reportedly decreased as a consequence of damage to the arachnoid. L-PGDS is a bifunctional protein, acting not only as a PGD2-producing enzyme but also as a lipophilic ligand carrier and a chaperone preventing amyloid $\beta(\mathrm{A} \beta)$ misfolding aggregation implicated in Alzheimer's disease. CSF A $\beta$ decrease was already reported in idiopathic NPH. The aim of this study was to confirm the L-PGDS decrease in idiopathic NPH and to clarify its relationship with symptoms.

Methods: We evaluated 24 patients (Age 75.7 7.7.8, M 11/F 13) referred for the ventriculomegaly with no CSF pathway obstruction, and conducted CSF tap test to determine the surgical indication. NPH symptoms were evaluated before and after tap test, using idiopathic NPH grading scale, MMSE, FAB and up and go test.

Results: 12 of 24 patients were diagnosed to have idiopathic NPH, supported by shunt or tap test response and typical MRI feature (disproportionately enlarged subarachnoid-space hydrocephalus; DESH). L-PGDS levels are significantly decreased in DESH patients compared to non-DESH patients ( $13.3 \pm 3.3$ vs $20.8 \pm 6.8 \mathrm{microg} / \mathrm{ml}, \mathrm{p}=0.009)$. Among clinical profiles, FAB significantly correlated with L-PGDS levels in whole patients (Spearman $r=-0.59, p=0.005$ ) and DESH patients (Spearman $r=-0.68, p=0.02$ ). Moreover, $\mathrm{L}$ PGDS levels showed positive correlation with FAB increase after treatment (Spearman $r=0.62, p=0.04$ ).

Conclusions: Our data not only support the diagnostic value of L-PGDS as a CSF biomarker, but also propose the potential role of this protein as a surrogate marker of frontal lobe dysfunction of NPH. 


\section{Presentation Number: 59}

\section{Subthalamic Nucleus Stimulation Reverses Spinal Motoneurone Activity In Parkinsonian Patients}

sylvie raoul, $\mathrm{MD}, \mathrm{PhD}$, chu nantes, nantes, France

Introduction: Rigidity is a cardinal symptom of Parkinson disease in which motoneurone responses to muscle afferent volleys are involved since it is suppressed after dorsal root section. In healthy subjects, motoneurone responses muscle afferents exhibit a frequency related depression: the greater the stimulus rate, the smaller the motoneurone responses.

We investigated this frequency related depression in Parkinsonian patients undergoing deep brain stimulation.

Methods: Nine parkinsonian patients with chronic bilateral STN stimulation associated with L-dopa treatment and exhibiting rigidity and akinesia, but no or little tremor were investigated together with nine aged-matched healthy subjects. The effects of repetitive stimulation of muscle afferents (homosynaptic depression) onto motoneurones innervating the wrist flexors (FCR) and the ankle extensors (Sol) using $\mathrm{H}$ reflexes evoked at $0.5 \mathrm{~Hz}$ and $0.125 \mathrm{~Hz}$ were tested in the right side of healthy subjects and in the more rigid side of parkinsonian patients in four situations: i) ON-ON, i.e. with deep brain stimulation and L-dopa uptake; ii) ON-OFF, with Ldopa uptake and without deep brain stimulation; iii) OFF-ON, without L-dopa uptake and with deep brain stimulation; iv) OFF-OFF, without any treatment.

Results: When the neurostimulation was OFF, the frequency related depression was completely suppressed and the patient rigid. When the neurostimulation was $\mathrm{ON}$, the level of frequency related depression was restored and the rigidity almost suppressed. These results suggest that frequency related depression is controlled by descending pathways. We interpret this unexpected finding, taking into account the capacity of neuromodulatory input originating from the brainstem to boost spinal motoneurone firing. Conclusions: it may be argued that a neuronal circuit revealed in chronic patients may not be functional in healthy subjects, the present results suggest for the first time in humans, that neuromodulatory inputs originating from the brainstem influence the behavior of spinal motoneurones and thus control of muscle tone 
Presentation Number: 118

\section{Different Performance Between Food Reaching Motors And Finger Precise Motors Ameliorated By STN-DBS In MPTP- treated Monkeys.}

Tetsuya Asakawa, MD, PhD, Hamamatsu University, School of Medicine, Hamamatsu, Shizuoka, Japan; Kenji Sugiyama, MD, PhD, Hamamatsu, Shizuoka, Japan; Takao Nozaki, MD, Hamamatsu, Shizuoka, Japan; Hiroki Namba, MD, PhD, Hamamatsu, Shizuoka, Japan

Introduction - Gross motor and precise motor are controlled by different neural circuits. This study is designed for quantitative evaluation the efficacy of deep brain stimulation of the subthalamic nucleus (STN-DBS) in improving the gross motor and precise motor in MPTP treated monkeys.

Methods - Three MPTP treated monkeys were involved in this study. We used Food Reaching Test (FRT) to evaluate gross motor, while Gripping Test (GT) to precise motor. Levodopa (L-dopa) test was performed first to confirm the practicability of these tasks along with the response of dopamine administration. We then compared the improvements of the gross motor and finger precise motor conducted by STNDBS.

Results - L-dopa test showed significant improvements in both FRT and GT ( $\mathrm{p}<0.01$, reached a normal level). Therefore the practicability of these tasks and good response of L-dopa administration were confirmed. Interestingly, DBS significantly ameliorated the gross motors (food reaching motors) tested by FRT ( $<0.01$, not reach a normal level), however, did not show good efficacy for finger precise motors tested by GT $(\mathrm{p}>0.05)$.

Conclusion - STN-DBS showed poor improvements for finger precise motors. We are now planning to confirm these results also in clinical DBS patients, and the mechanisms should be explored by bench studies in the future. 
Isolation And Transplantation Protocol Of Human Fetal Neural Progenitor Cells In The Treatment Of Huntington'S And Parkinson'S Disease.

William O. Contreras Lopez, University Freiburg Medical Center, Freiburg im Breisgau, Germany

Neurodegenerative diseases are each time more prevalent, since populations are aging older due to the advances of science and medicine related disciplines, enlarging the window of time possibility to develop one of these catastrophic diseases. Cell replacement therapies were born hoping to become therapeutic modalities aiming to improve patient's lose functions by replacing the damaged or loss cell population.

Here we present a step wise practical detailed description of the relevant surgical anatomy to select the transplanted tissue in HD and $\mathrm{PD}$, as well as the description of the in vitro propagation and differentiation of human fetal neural precursor cells, with emphasis on region-specific characterization of the human fetal neural tissue and primary cultures and the Long-term expansion of the region specific human fetal-derived cultures. Aiming for an optimal anatomic understanding of the micro-dissection respecting the limits between the main cell populations necessary to avoid the development of graft-induced undesired secondary effects, and to investigate the influence of prolonged in vitro cultivation on the maturation process of the embryonic tissue.
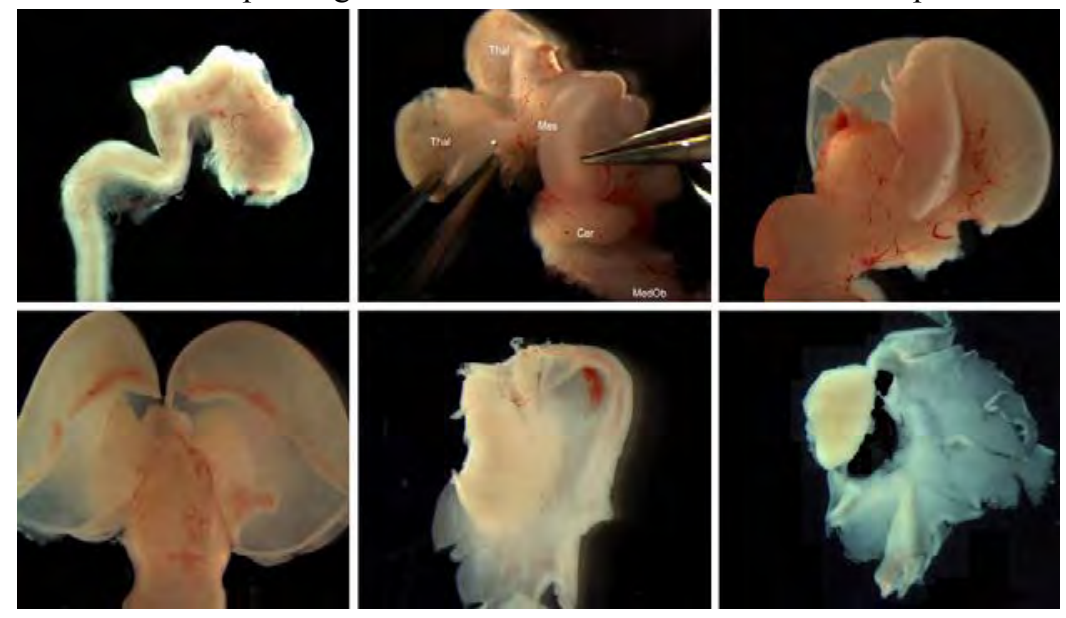


\section{Session Title: Flash Session - From the Laboratory to the Clinic}

Session Time: Wednesday, May 29, 2013, 3:30 pm - 4:30 pm

Presentation Number: 361

\section{A Novel Intraoperative Method for Monitoring Hyperacute Changes in Cerebral Ischemia}

Hiroshi Fujioka, MD, PhD, Kanmon Medical Center, National Hospital Organization, Yamaguchi, Japan; Seiji Uesugi, MD, PhD, Yamaguchi, Japan; Akifumi Izumihara, MD, PhD, Yamaguchi, Japan; Hideki Harada, MD, PhD, Kurume University School of Medicine, Fukuoka, Japan; Katsuhiro Yamashita, MD, PhD, Yamaguchi, Japan

INTRODUCTION: We have previously reported cerebral hyperexcitability in rats through excitatory-inhibitory imbalance after $1 \mathrm{~h}$ of ischemia (Fujioka et al. Stroke 35: e346-, 2004). However, it was unclear whether such an imbalance is induced in the hyperacute stage of ischemia in humans. We addressed the issue in patients undergoing carotid endarterectomy (CEA), wherein cerebral ischemia is induced by temporary carotid occlusion.

Methods: Informed consent was obtained from all patients with indication of CEA (symptomatic patients with $>70 \%$ stenosis; $\mathrm{n}=6$, mean age; 72.3 years). Routine shunting was used, and temporary occlusion was applied twice (cf. occlusion (1) and (2) in Figures 1 and 2). Short-term somatosensory evoked potentials (SSEPs) were evoked by paired-pulse stimuli with an inter-stimulus interval of 30 ms, and each peak-to-peak amplitude was measured by using the N20/P25 complex. The excitability index $Q$ was calculated by the amplitude of the second stimulus divided by the first. Near-infrared spectroscopy monitoring was also used to monitor regional cerebral saturation $\left(\mathrm{rSO}_{2}\right)$.

Results: Compared to each pre- and post-occlusion period, $Q$ values significantly increased during the second occlusion and reversibly decreased when the occlusion was released (Tukey's post hoc tests; ${ }^{* *} \mathrm{p}<0.01$; Figure 1); however, occlusion did not induce significant changes in amplitudes by traditional single-pulse SSEPs $(\mathrm{p}=0.188$ by repeated-measures analysis of variance; Figure 2). Asymptomatic cerebral infarct was post-operatively recognized in 1 patient with an increased $Q$ value $(Q=1.1)$ and a substantial $46.9 \%$ reduction of single-pulse SSEP amplitude. Occlusion did not induce significant changes in $\mathrm{rSO}_{2}$.

CONCLUSION: We demonstrated hyperacute ischemia-induced hyperexcitability for the first time in humans. Although single-pulse SSEPs were useful in detecting ischemia, paired-pulse SSEPs proved more reliable in detecting ischemia-induced neuronal imbalance, suggesting that it could be a prognostic monitoring tool for CEA and other ischemia-related cerebrovascular diseases.
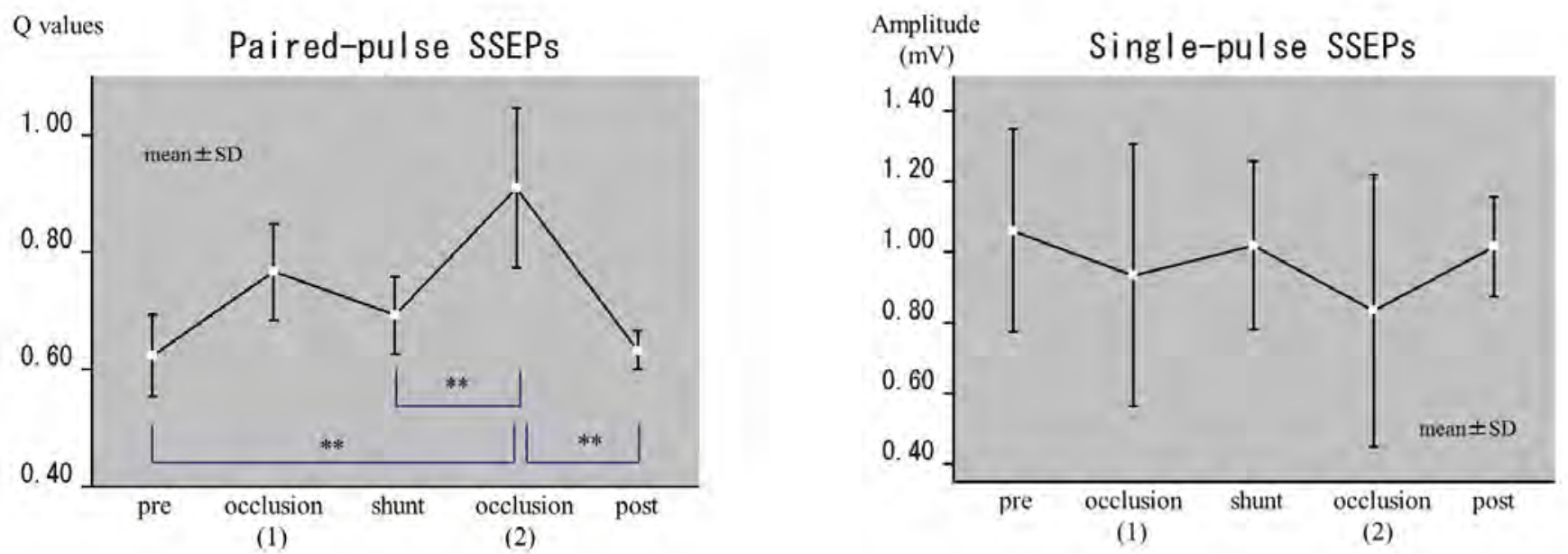


\section{Session Title: Flash Session - From the Laboratory to the Clinic}

Session Time: Wednesday, May 29, 2013, 3:30 pm - 4:30 pm

Presentation Number: 376

\section{Neurophysiological Characteristics Of Subthalamic Neurons Receiving Inputs From The Limbic Cortex}

Sumito Sato, MD, Kitasato University School of Medicine, Sagamihara, Japan; Satomi Chiken, PhD, Okazaki, Japan; Futaba Maki, MD,PhD, Kawasaki, Japan; Kiyotaka Fujii, MD,PhD, Sagamihara, Japan; Atsushi Nambu, MD,PhD, Okazaki, Japan

Introduction: Deep brain stimulation targeting the subthalamic nucleus (STN) ameliorates motor disability of Parkinson's disease, whereas it also sometimes induces emotional changes such as hypomania or depression. It has been assumed that electrical stimulation spreads to the limbic part of the STN. We investigated neurophysiological characteristics of STN neurons receiving inputs from the limbic cortex to study emotional functions of the cortico-basal ganglia circuitry.

Methods: Two Japanese monkeys were used in this study. Stimulating electrodes were implanted chronically in three cortical areas: forelimb regions of the primary motor cortex (MI) and the supplementary motor area (SMA), and the prelimbic area. Extracellular unit activities of STN neurons were recoded under awake state, and their responses to the cortical stimulation were examined.

Results: The STN neurons were divided into three groups based on responses evoked by cortical stimulation: MI-, SMA-, and prelimbic-recipient neurons. These three groups showed similar spontaneous firing rates and patterns. MI-, SMA-, and prelimbic stimulation induced biphasic responses composed of early and late excitations. The latencies and durations of early and late excitations induced by prelimbic stimulation were longer than those by MI- and SMA-stimulation. Prelimbic-recipient neurons were located in the most medial region of the STN, while MI- and SMA-recipient neurons were found in the lateral and middle portions, respectively.

Conclusions: The cortico-basal ganglia circuitry processes information from the limbic cortex in the similar manner to that from the motor cortices, however processing speed of limbic system may be slower. 


\title{
Session Title: Flash Session - From the Laboratory to the Clinic
}

Session Time: Wednesday, May 29, 2013, 3:30 pm - 4:30 pm

Presentation Number: 49

\author{
Anatomic Study of Fiber Tracts Linking the Basal Ganglia and Cerebellum in Patients with Parkinson's Disease \\ Jennifer A. Sweet, MD, University Hospitals Case Medical Center, Cleveland, OH; Benjamin L. Walter, MD, Cleveland, OH; \\ Jonathan P. Miller, MD, Cleveland, $\mathrm{OH}$
}

Introduction: Stimulation of white matter tracts in the vicinity of targeted structures may contribute to the observed therapeutic effects of deep brain stimulation for Parkinson's disease (PD). Two tracts linking the basal ganglia and cerebellum have been described in primates: the subthalamo-ponto-cerebellar tract (SPCT) and dentato-rubro-thalamic tract (DRTT). We used fiber tracking to evaluate white matter tracts that connect the cerebellum to the region of the basal ganglia in patients with PD who were candidates for DBS.

Methods: Fourteen patients with advanced PD underwent high-resolution MR imaging including DTI, nine of whom subsequently underwent DBS of the subthalamic nucleus. For each side in each patient, two regions of interest were defined encompassing: (1) the region of the basal ganglia including the red nucleus and subthalamic nucleus, and (2) the contralateral cerebellum. Fiber tracking was performed using iPlan FiberTracking (BrainLab, Feldkirchen, Germany) software. The size of each tract was measured, and for patients who underwent implantation, the location of the tract relative to the cathodal electrode contact was determined.

Results: In every patient, two distinct white matter tracts were identified that corresponded to the described anatomic features of the SPCT and DRTT, respectively. In nine patients who presented with tremor, a significant relationship $(p<0.001)$ was found between the side with the larger DRTT and the side of greater tremor amplitude. In thirteen of the sixteen implanted electrodes $(81 \%)$, the active contact was traversed by the DRTT, and its average distance from the DRTT in the remaining three electrodes was $2.61+/-0.81 \mathrm{~mm}$. Conclusions: The SPCT and DRTT may be related to the expression of the symptoms of Parkinson's disease, and this may have implications for DBS targeting. 


\section{Session Title: Flash Session - From the Laboratory to the Clinic}

Session Time: Wednesday, May 29, 2013, 3:30 pm - 4:30 pm

Presentation Number: 116

Robot-assisted Guide Tube Implantation for Functional Neurosurgery - Analysis of Targeting Accuracy and Procedural Time Neil Barua, Frenchay Hospital, Bristol, United Kingdom; Max Woolley, PhD, Renishaw, United Kingdom; Stephen O'Sullivan, Renishaw, United Kingdom; Rob Harrison, Renishaw, United Kingdom; Catriona Fennelly, Renishaw, United Kingdom; Gavin Murray, Renishaw, United Kingdom; Steven Gill, FRCS, MS, Frenchay Hospital, Bristol, United Kingdom

Introduction: For over 10 years we have performed MRI-directed functional neurosurgery using implantable guide tubes delivered via the Leksell frame. In a large series of over 200 implantations, more than $96 \%$ of stylets were placed within $1.5 \mathrm{~mm}$ of the planned target using this frame-based method. In September 2012 we introduced a novel method of guide tube implantation using the neuromate ${ }^{\circledR}$ neurosurgical robot, which facilitates the drilling of a precise burr hole into which the guide tube hub push fits. The aim of this study was to compare targeting accuracy and procedural times for Leksell frame and robot-assisted guide tube implantation.

Methods: Data on targeting accuracy and procedural time was prospectively collected for 36 robot-assisted guide tube and stylet implantations. Twenty-four guide tubes were implanted for drug delivery through intraparenchymal microcatheters and 12 for DBS electrodes. Targeting accuracy was determined by measuring the distance between the planned target on a pre-operative CT angiogram, and the actual target on a peri-operative CTA. This distance was independently measured by 3 investigators in a trajectory view using neuroinspire ${ }^{\mathrm{TM}}$ stereotactic planning software, and the mean $( \pm \mathrm{SD})$ targeting error calculated. Targeting error and procedural time was compared to 36 consecutive guide implantations using the Leksell frame.

Results: There was a non-significant reduction in mean targeting error for robot-assisted guide tube implantation compared with the Leksell frame-based method $(0.81 \pm 0.48 \mathrm{~mm} \vee 1.03 \pm 0.41 \mathrm{~mm}, \mathrm{p}=0.11)$. However, the procedural time for each guide tube implantation was significantly shorter with the robot-assisted method $(22.5 \pm 12.9$ v $54.8 \pm 16.6$ minutes, $\mathrm{p}=0.002)$.

Conclusions: Use of the neuromate ${ }^{\circledR}$ robot for guide tube-directed functional neurosurgery delivers equal targeting accuracy to the Leksell frame with significantly reduced operating times. 


\section{Presentation Number: 70}

\section{Stereotactic Thalamic Vim-vcpc Stimulation for the Treatment of Cases with Central Post-stroke Pain}

Masafumi Hirato, M.D., Gunma University Graduate School of Medicine, Maebashi, Japan; Takaaki Miyagishia, M.D., Maebashi, Japan; Yuhei Yoshimoto, M.D., Maebashi, Japan

Introduction: We studied the effectiveness of thalamic ventralis intermedius (Vim) - ventralis caudalis parvocellularis (Vcpc) stimulation for pain relief in cases with central post-stroke pain (CPSP). Based on the data of intraoperative thalamic microrecording and microstimulation study and preoperative PET study, we also studied the possible pathophysiological changes of thalamo-cortical system in the genesis of CPSP.

Methods: Stereotactic thalamic Vim-Vcpc stimulation was indicated for 7 cases with CPSP. They showed diffuse intractable pain on the affected hemibody. Intraoperative thalamic microrecording and microstimulation study using recording electrode were performed in all cases, preoperative PET study in 6 cases, and postoperative test stimulation study using therapeutic electrode in 5 cases.

Results: Long-lasting good pain relief was achieved in 4 cases and moderate in 3 cases, though postoperative acute test stimulation usually induced uncomfortable heaviness or numbness on the affected limbs. Neuronal activity increased around CVD lesion on the sensory thalamus, which associated with irregular long-duration burst discharges, in 5 out of 7 cases. In the other 2 cases, it was also electrically unstable. Preoperative PET study revealed high accumulation spot of FDG on the affected sensory thalamus in 3 out of 6 cases. Sensory responses in the thalamus to peripheral natural stimulation were recognized in 4 cases. Pain relief was recognized during chronic thalamic stimulation on the rostrodorsal part of surrounding area of the CVD lesion, where irregular burst discharges were frequently found. On the ventral part of this area, where corresponds to thalamic Vim-Vcpc nucleus, sensory responses were often encountered.

Conclusions: Sufficient and long-term pain relief was obtained using thalamic Vim-Vcpc stimulation in cases with CPSP. Focal hyperactivity or electrical unstability around the CVD lesion in the affected sensory thalamus might play important roles in the genesis of CPSP. 


\section{Presentation Number: 48}

\section{Testing The Opioidergic Hypothesis of PAG-DBS for Pain: A [11c] Diprenorphine PET Imaging Study}

Hugh P. Sims-Williams, MBChB MRCS, Neurosurgery, North Bristol NHS Trust, Bristol, United Kingdom; Shazia Javed, MbChB MRCS, Neurosurgery, North Bristol NHS Trust, United Kingdom; Julian C. Matthews, PhD, Manchester Academic Health Science Centre (MAHSC), United Kingdom; Sarah Love-Jones, BSc, FRCA, Anaesthesiology, North Bristol NHS Trust, United Kingdom; Peter S. Talbot, MD MRCPsych, Manchester Academic Health Science Centre (MAHSC), United Kingdom; Anthony E. Pickering, PhD FRCA, School of Physiology, University of Bristol, United Kingdom; Nik K. Patel, MD FRCS (SN), Neurosurgery, North Bristol NHS Trust, United Kingdom

Introduction: Deep Brain Stimulation (DBS) of the Peri-Aqueductal-Gray (PAG) was originally proposed to produce analgesic effects by the release of endogenous opioids(1)(2). This hypothesis is contentious in man and we aim to directly test it in man using Positron Emission Tomography (PET) with the radio-labelled opioid antagonist [11C] diprenorphine as a tracer to see whether we can show a regional pattern of evoked-opioid release.

Methods: Participants $(n=6)$ were selected from a "responder" cohort of patients implanted with PAG DBS for the treatment of deafferentation pains: phantom limb pain, brachial plexus avulsion and anaesthesia dolorosa.. Using [15O] water and [11C] diprenorphine radiotracers and a High Resolution Research Tomograph (Siemens, Knoxville, TN) PET camera we aim to quantify: i) regional cerebral blood flow changes; and ii) changes in the opioid receptor binding on stimulation of the PAG.

Results: We hypothesise that stimulation of PAG will result in endogenous opioid release and a reduction in diprenorphine-receptor binding in the midbrain and pain matrix. The study is underway with completion of recruitment due in March 2013.

Conclusions: De-afferentation pain causes significant morbidity, and DBS can provide $60-80 \%$ relief in some patients for a period of time(3). There is limited understanding of the principle neurotransmitter mechanisms and neural circuits engaged on stimulation of PAG(4). We anticipate that Diprenorphine PET will provide novel insights to optimise DBS for pain and may assist in the design of adjuvant therapies to prolong stimulation efficacy.

1. Akil et al. Antagonism of stimulation-produced analgesia by naloxone, a narcotic antagonist. Science. 1976;191(4230): 961-2.

2. Akil et al. Enkephalin-like material elevated in ventricular cerebrospinal fluid of pain patients after analgetic focal stimulation. Science. 1978;201(4354): 463-5.

3. Bittar et al. Deep brain stimulation for pain relief: A meta-analysis. J Clin Neurosci. 2005;12(5): 515-9.

4. Bittar et al. Deep Brain Stimulation for phantom limb pain. J Clin Neurosci. 2005 May;12(4): 399-404. 


\section{Deep brain stimulation in Short Lasting Unilateral Headache with Conjunctival Injection and Tearing (SUNCT)} Sarah Miller, MBBS, BSc, National Hospital for Neurology and Neurosurgery, London, United Kingdom; Fahid Rasul, London, United Kingdom; Susie Lagrata, London, United Kingdom; Marwan Hariz, London, United Kingdom; Ludvic Zrinzo, London, United Kingdom; Manjit Matharu, London, United Kingdom

Introduction: SUNCT is a primary headache syndrome characterised by short lasting attacks of unilateral pain accompanied by prominent lacrimation and redness of the ipsilateral eye. SUNCT is refractory to medical treatments in a small minority of patients. Neuroimaging studies suggest a role for the posterior hypothalamic region $(\mathrm{PH})$ in its pathogenesis. Previous reports on PH-deep brain stimulation (DBS) for SUNCT are limited to three patients 1-3. We present data on four more patients with medically refractory SUNCT who received PH-DBS.

Method: Four SUNCT patients underwent PH-DBS with an MRI-guided and MRI-verified approach without microrecording. The target on stereotactic T2-weighted images lay between the mammillothalamic tract and the anteromedial quadrant of the red nucleus. Details of headache characteristics and adverse events were recorded.

Results: All leads were within 1.0mm of the intended target point on postimplant stereotactic imaging. Stimulation was monopolar, with $1.3-3 \mathrm{~V}$ at $185 \mathrm{~Hz}$ and $60 \mu$ s throughout the follow-up period. The median follow up period was 22 months (range 9-34 months). Two patients had pure SUNCT and two multiple headache types (additional cluster headache and migraine). Median improvement in headache load \{defined as the [severity (on the visual analogue scale)] x [duration] x [frequency] of headache attacks\} was $84 \%$ (range 74-100\%). Median subjective improvement was 70\% (range 20-100\%). Patients with pure SUNCT fared better than those with mixed headaches (mean improvement $92.5 \%$ vs. $78.5 \%$ ). No sustained side-effects related to stimulation were reported.

Conclusion: PH-DBS may be a useful treatment in refractory SUNCT. Our series suggests those with a pure headache syndrome may benefit more than those with mixed headaches.

1. Leone, M. et al. Ann Neurol 57, 924-927 (2005).

2. Lyons, M. et al. J Neurosurg 110, 279-281 (2009)

3. Bartsch, T. et al. Cephalalgia 31, 1405-1408 (2011). 
Presentation Number: 303

\section{Implanted Occipital Nerve Stimulation Results for Intractable Headache Predicted by Transcutaneous Electrical Nerve Stimulation}

Vincent ROUALDES, MD, University Hospital Nantes, Nantes, France; Jean-Paul Nguyen, MD,PhD, Nantes, France; Julien Nizard, MD, Nantes, France

Introduction: Implanted electrode occipital nerve stimulation is a technique that is now regularly proposed to patients with medically intractable chronic headache. However, no element predictive of the result of this operation has been demonstrated up until now.

Methods: The authors report a series of 33 patients (occipital neuralgia $(n=15)$, cervicogenic headache $(n=7)$, cluster headache $(n=6)$, chronic migraine $(\mathrm{n}=5)$ ), who underwent preoperative transcutaneous electrical nerve stimulation (TENS) using electrodes placed over the future implantation site and who were tested for 1 to 3 months. Patients who considered that they were improved by TENS were operated by implantation of occipital nerve stimulation (ONS) electrodes.

Results: TENS was found to be remarkably effective (72.3\% improvement of the VAS). The long-term postoperative (ONS) results (mean follow-up: 36 months) showed a significant reduction of the number of headache days per month, and mean and maximum VAS scores in all patients $(\mathrm{p}<0.001)$. Postoperative improvement was significantly better in patients who obtained a more marked improvement by TENS ( $\mathrm{p}=0.01$ ), strongly suggesting that TENS is predictive of the results of the operation. No significant difference was observed according to the type of headache.

Conclusions: 1. Patients operated by implanted occipital nerve stimulation obtained significant long-term improvement. 2. The results of TENS placed over the zone of the superficial occipital nerve were predictive of the results of implanted electrode occipital nerve stimulation. The predictive value of TENS is particularly useful in patients with chronic migraine, in whom it may be difficult to determine the indication for implanted occipital nerve stimulation. 
Presentation Number: 324

Pulsed Radiofrequency Of Sympathetic Lumbar Plexus Versus Sympathetic Block In The Management Of Lower Limb Complex Regional Pain Syndrome Type 1

Tiago S. Freitas, Brasília University Hospital, Brasília, Brazil; Renato Deusdará, Brasília, Brazil; Iruena Kessler, Brasília, Brazil

Introduction: Complex Regional Pain Syndrome (CRPS) type 1 is a neuropathic pain syndrome which clinical treatment involves oral medications, physiotherapy and other alternative therapies. The purpose of this study was to compare two safety options: pulsed radiofrequency( PRF) or sympathetic blocks (SB), and their efficacy in the different aspects of this neuropathic pain and in quality of life of patients suffering from this disease.

Methods: 40 randomized patients to receive PRF or SB in lower limb CRPS type 1 . They were prospectively evaluated with VAS scores, neuropathic pain scale and RAND SF-36 (Research and Development Short Form Health Survey) in a follow up of 7 days, 3 6-12 and 15 months.

Results: There were similar reductions from baseline in various pain scores. In the PRF group these results were statistically more consistent with the follow up period with the burning pain $(\mathrm{P}<0.05)$. The other pain parameters and RAND SF-36 scale had similar results in both groups.

Conclusion: CRPS type 1 is a neuropathic pain syndrome usually of difficult treatment and many patients evolve to the necessity of interventional procedures. PRF has gained evidence in pain management due a possible neuromodulation effect. PRF appears as a technique with similar results when compared with the sympathetic block. Only one pain outcome (hot pain) was statistically significant and this difference was insignificant to the final result. Once a higher-cost procedure with too few benefits, this particular difference did not affect the quality of life (RAND SF-36). 
Presentation Number: 296

Intrathecal Ziconotide (ITZ) For The Treatment Of Sublesional Neuropathic Pain In Patients With Spinal Cord Lesions. Patrick MERTENS, MD, PhD, University LYON 1, LYON, France; Andre BRINZEU, MD, LYON, France; Gustavo POLO, MD, LYON, France; Emile Simon, MD, LYON, France

Introduction: The goal of this study was to evaluate the efficacy and safety of ITZ in patients with sublesional neuropathic pain following spinal cord lesions.

Methods: Eight patients with severe refractory neuropathic pain following spinal cord injury (SCI) of various etiologies were considered for ITZ. The efficacy of the drug was tested initially by bolus injection $(1.5,2$ and $2.5 \mathrm{mcg})$ and/or continuous infusion (increased progressively by $0.6 \mathrm{mcg}$ every 48 hours). Creatine-phospho-kinase (CPK), blood pressure, heart rate, temperature and respiratory rate with neurological and psychological status were followed throughout the tests. Patients were considered responders if a $>30 \%$ reduction in baseline VAS was observed without side-effects and a pump placement with continuous intrathecal infusion was then proposed. Detailed analysis of test failures were performed in order to identify reasons for lack of efficacy.

Results: 5 patients were responders during the tests with a mean maximal VAS decrease of $67 \%$.

One of these patients who benefit from ITZ had intolerably high CPK levels and therefore was not implanted.

Another patient developed episode of acute urinary retention following a bolus test and did not accept the pump implantation.

Twelve months follow up of the three patients that were implanted shows a decrease of $61 \%$ in VAS with an average ITZ dosage of 4.2 mcg per day.

Of the three non-responder patients, two had a contrast myelogram showing a partial or total block at the lesion level.

Conclusions: Our data demonstrates an important decrease in VAS after ITZ for well selected patients with SCI. But important adverse reaction made impossible the use of this treatment in an important proportion of patients $(25 \%$ of those tested and $40 \%$ of responders). Meanwhile, this study with a short population is encouraging as facing with one of the most difficult to treat neuropathic pain. 
Presentation Number: 256

\section{Open Thoracic Cordotomy for pain: Lost art or a reviving skill?}

Naeem A. Farooqi, FRCS(NeuroSurg), Walton Centre for Neurology \& Neurosurgery, Liverpool, United Kingdom; Jonathan Ellenbogen, MRCS, Liverpool, United Kingdom; Matthew G. Stovell, MBBS,BSc,MRCS, Liverpool, United Kingdom; Jibril Osman Farah, FRCS(NeuroSurg), Liverpool, United Kingdom; Paul R. Eldridge, FRCS, Liverpool, United Kingdom

Introduction: Cancer pain within palliative care setting is a difficult condition to treat. WHO analgesic ladder for palliative care is the gold standard for cancer pain treatment however ablative techniques may be more appropriate for patients with focal pain in the context of advanced malignancy. The effectiveness of percutaneous cervical cordotomy is well established; however open cordotomy, though indicated relatively infrequently also provides a potent method for controlling pain in these patients, can be performed bilaterally with less morbidity than is appreciated.

Methods: To illustrate the utility of open thoracic cordotomy for refractory cancer pain in a series of 4 cases, in two of which bilateral open cordotomy was performed are presented.

Results: All 4 cases suffered from debilitating back and bilateral leg pain using maximal analgesia. 3 had percutaneous cervical cordotomy performed previously, unilaterally on the contralateral side. The fourth case - a bilateral cordotomy - had an intrathecal drug delivery system in place for the previous 12 months, and had undergone percutaneous cervical cordotomy unilaterally, which had been successful for few months, following which the pain had recurred. The patient with metastatic GBM underwent open bilateral cordotomy as primary procedure due to the presence of cervical metastases.

All patients reported excellent pain relief. The analgesic requirements decreased significantly with concomitant reduction in side effects contributing to improved quality of life. There was minimal procedural morbidity. Pain relief was sustained in 3 patients prior to their demise from systemic disease; one is still alive and pain free.

Conclusions: Open cordotomy, whilst only rarely indicated, is an effective procedure for the focal pain of malignancy; earlier intervention than suggested by the WHO analgesic ladder should be considered in cases with focal pain from malignancy in the light of these excellent outcomes, and avoids the toxic side effects of systemic medication. 


\section{Long term Outcomes for the Use of Dorsal root entry zone (DREZ) Lesioning for Brachial Plexus Avulsion Injury} Jonathan R. Ellenbogen, BMedSc (Hons) MBChB with Honours MRCS, The Walton Centre NHS Foundation Trust, Liverpool, United Kingdom; Naeem Farooqi, FRCS, Liverpool, United Kingdom; Radhika Manohar, Liverpool, United Kingdom; Jibril OsmanFarah, FRCS, Liverpool, United Kingdom; Paul R. Eldridge, FRCS, Liverpool, United Kingdom

Introduction: Pain associated with brachial plexus avulsion injury, in which the upper limb is both insensate and flail, maybe severe and intractable. It does not appear responsive to neuromodulatory techniques. It can be treated highly effectively by dorsal root entry zone (DREZ) lesioning. Although unusual for ablative treatments for chronic neuropathic pain states to be long lasting DREZ lesioning in these specific circumstances appears an exception. The technical procedure can be demanding as distorted anatomy following injury can make identification of the DREZ difficult. This series demonstrates that good long term outcomes can be obtained, and that neurophysiological techniques maybe helpful in identifying the DREZ.

Methods: Retrospective case review of patients who underwent DREZ lesioning for intractable brachial plexus avulsion pain at a single institution over a 6-year period (2006-2012).

Results: 10 patients were identified. All had flail and insensate limbs, with four having amputations for this reason. DREZ lesioning was performed using thermode controlled radiofrequency ablation, with intra-operative neurophysiological monitoring to aid localisation.

Age range 33 - 64 years, with a follow up range of 3 months to 5 years. Previously five patients had undergone spinal cord stimulation (SCS) and one patient had undergone motor cortex stimulation, deep brain stimulation and SCS all with no benefit. 9 patients obtained excellent (over 80\%) pain relief. There were no pain relapses. Complications were minor but included cervical pain/kyphosis in one patient; minor leg weakness in two patients; reduced proprioception in the leg in three patients; post-operative sepsis in another. Conclusions: DREZ lesions can achieve excellent and long lasting pain relief in well defined cases of brachial plexus avulsions. Although DREZ lesioning carries a risk of serious complications, these are in fact rare. 
Presentation Number: 239

\section{Motor Cortex Stimulation For The Treatment Of Severe Pain}

Vladimir A. Shabalov, PhD, The N.N.Burdenko Institute of Neurosurgery of RAMS, Moscow, Russian Federation; Emil Isagulyan, The N.N.Burdenko Institute of Neurosurgery of RAMS, Moscow, Russian Federation; Maria A. Khitj, Moscow, Russian Federation

Objectives. Our goal to present the outcomes of MCS in the complex treatment of deafferentation type of neuropathic pain in our clinic.

Material and methods. 23 patients (12 males and 11 females, aged 29 - 56 years) were undergone to implantation of epidural electrodes over the central sulcus region since 2005 until 2011 years. Six patients had facial anesthesia dolorosa related to different destructive procedures on the trigeminal nerve branches, 7 patients had severe deafferentation pain in the arm due to traumatic brachial plexus injury, 3 patients suffered from poststroke pain syndrome (PSP). Another 6 patients had phantom limb pain. Finally one patient (female) suffered from reffered pain due to traumatic spinal cord injury (C6 fracture) with tetraparesis. In case of positive trial stimulation in 20 of them the neurostimulator was subcutaneously implanted.

In all patients repetitive transcranial magnetic stimulation (rTMS) of central cortex contralateral to the pain territory was performed as a trial preoperative test. Pain intensity was evaluated with Pain and Quality of Life Card (PQLC) based on 10-point Visual Analog Scale (VAS).

Results. Good outcomes were achieved in 17 patients both in immediate postoperative period and in long-term (up to 48 month) follow-up. Pain had improved by 35 to $70 \%$ in comparison with baseline. All 17 patients have stopped to use opiates. Loss of MSC effect was observed several months later in 3 cases: one patient with traumatic spinal cord injury and two patients with complete avulsion of brachial plexus.

Conclusions. Motor cortex stimulation is effective technique for the treatment of severe neurogenic pain. Preoperative rTMS outcomes in general correspond to ones of chronic MCS. It is necessary to make more researches, for more exact analysis of efficiency MCS in the treatment of severe neuropathic pain syndromes. 
Presentation Number: 339

The Effect Of Epidural Ozone Therapy In Patients With Chronic Pain Related To Failed Back Surgery Syndrome.

Erich T. Fonoff, MD, PhD, University of Sao Paulo, Sao Paulo, Brazil; Francisco N. Magalhaes, MD, Sao Paulo, Brazil; Manoel J.

Teixeira, MD, PhD, Sao Paulo, Brazil

Introduction: In the last two decades, ozone has emerged as a treatment for low back pain, applied by means of minimally invasive techniques.

Objective: The aim of this study is to assess the effect and safety of ozone therapy applied in the epidural space for chronic pain related to failed back surgery syndrome.

Methods: The investigators studied 13 sequential patients of both sexes, between 18 and 70 years old with persistent chronic pain (more than six months) in lumbar region and in the lower limb related to failed back surgery syndrome (FBSS). Pain was classified in neuropathic and non-neuropathic regarding the topography (lumbar and lower limb). The patients received the ozone gas in the lumbar epidural space via spinal-sacral endoscopy. Clinical evaluation was performed before, right after (24 hours) and 1, 3 and 6 months after intervention with visual analogue scale and Oswestry Disability Index (ODI).

Results: Overall, the patients had $43.7 \%$ reduction of lumbar pain, $60.9 \%$ reduction in leg pain in six months followed by $44.0 \%$ of improvement in ODI. The reduction of pain and in the disability index was markedly greater in patients with non-neuropathic predominant pain, $95.2 \%, 80.6 \%$ and $75.3 \%$ improvement in lumbar, leg pain and ODI respectively, while neuropathic predominant pain patients experienced only $12.5 \%, 42.4 \%$ and $20.9 \%$ improvement, also respectively. No neurological or infectious complications were observed acutely or during the follow-up. Conclusion: The present data suggests that epidural ozone might be a therapeutic option for persistent low back pain, especially in non-neuropathic predominant pain patients, but double-blind controlled studies are still required to prove its efficacy. 
Presentation Number: 189

\section{Combined fMRI, SSER, And Intraoperative Stimulation For Identification Of Cortical Areas In Motor Cortex Stimulation For Pain}

Tommy Bergenheim, MD, PhD, Dept of Neurosurgery, Umeå, Sweden; Erik Nordh, MD, PhD, Umeå, Sweden; Lars Nyberg, PhD, Umeå, Sweden; Johan Eriksson, PhD, Umeå, Sweden; Anna Birkegård, Umeå, Sweden; Kristina Edvardsson, Umeå, Sweden

Background: Identification of cortical areas is crucial for optimal motorcortex stimulation. This can be achieved by imaging techniques, by electrophysiological recording/stimulation (NP), or by functional imaging (fMRI). This pilot study evaluates motor cortex mapping by comparing functional imaging and electrophysiological stimulation.

Methods: Six patients were examined with 3T MRI using fMRI BOLD imaging protocols for activating facial, tongue and hand motor areas. fMRI statistical maps were merged with anatomical MR images and imported into a Stealth navigation system for intraoperative orientation. Cortical SEP-recordings and transdural cortical stimulations were done through a surface electrode grid, centred over the sulcus centralis (SC) and needle electrodes for EMG in muscles of interest. The grid position was related to the MRI/fMRI images by neuronavigation. The SC was identified by anatomical landmarks and median nerve SEP phase reversal. Optimal NP stimulation areas for various muscles were defined via the electrode grid. All areas outlined by NP were finally compared to the corresponding fMRI-defined areas.

Results: Transdural stimulations identified localised spots with maximum muscle responses properly related to the cortical areas of the hand, arm, and face. Overall, there was a good correspondence between the areas defined by cortical stimulation and fMRI, but smaller and more circumscribed areas were defined by fMRI. In all patients, hand muscles were activated from more extensive cortical areas than seen for other muscles during NP stimulations. In a few cases, the course of the SC differed, with a maximum difference of $10 \mathrm{~mm}$ between the MRI and the NP assessed locations.

Conclusion: In this pilot study we defined cortical areas both with fMRI integrated into a neuronavigation system and NP, and found a good correspondence between methods. A higher degree of accuracy and safety can likely be achieved by combining the methods. 
Presentation Number: 369

\section{Peripheral Field Stimulation For Chronic Focal Pain}

Milind Deogaonkar, M.D., Ohio State University Medical Center, Columbus, OH

Introduction: Although peripheral nerve stimulation (PNS) is often employed for the treatment of a variety of chronic intractable pain syndromes, field stimulation (PFS) is gaining increasing popularity. The author presents his experience, complications, and outcomes associated with PNS and PFS for the treatment of various chronic pain syndromes.

Methods: This is a retrospective review of 150 patients undergoing PFS surgery from 2007 to 2012 at the Cleveland Clinic. Patients had a variety of diagnoses including focal neuralgias to deafferentation pain to back pain. Patients were treated with subcutaneous electrode placement below or around the area of maximum pain under direct fluoroscopic visualization for PFS. Patients' pain visual analog scores (VAS) were studied during the preoperative, immediate postoperative and last follow-up visits.

Results: Patients had improvement in their VAS pain scores in all categories studied. Pain resolution was noted by $68 \%$ immediate post-op and 55\% at the six month follow-up period. In patients with post-intervention trigeminal neuropathic pain the improvement was remarkable (75\%). PFS for peripheral neuropathic pain and back pain also showed $64 \%$ improvement in the VAS. There was corresponding decrease in the pain medication intake as well. Complications were rare but included wound dehiscence, lead malfunction, lead migration and erosion through the skin.

Conclusions: In conclusion, PFS is a safe and effective alternative for patients with intractable chronic pain that have attempted various other treatments. 


\section{Presentation Number: 40}

\section{Selective Dorsal Rhizotomy for Neoplastic lumbosacral plexopathy in terminal pelvic cancer}

Byung-chul Son, MD, PhD, St. Vincent's Hospital, The Catholic University of Korea, Suwon, Kyeonggi-do, Korea, Republic of; Deog-ryeong Kim, MD, Suwon, Kyeonggi-do, Korea, Republic of; Sang-Won Lee, MD, PhD, Suwon, Kyeonggi-do, Korea, Republic of

Introduction: Neoplastic lumbosacral plexopathy is characterized by severe and unremitting pain that is resistant to conventional treatment and by a short survival ( $<6$ months) from the time of diagnosis in advanced pelvic cancer. According to a recent review of destructive procedures for control of cancer pain, only cordotomy has shown to play an important role in the treatment of cancer pain. To date, the effectiveness of dorsal rhizotomy, which selectively interrupts pain transmission, has not been reported in neoplastic lumbosacral plexopathy. The authors performed selective dorsal rhizotomies (SDR) in the involved segments for intractable pain of neoplastic lumbosacral plexopathy because the aim of pain surgery in this condition is to interrupt the transmission of pain, not to make the painful areas analgesic, and since the estimated life expectancy is short, therefore recurrence could be minimal even with ablation of posterior roots.

Methods: SDR of the involved segments were performed in six cancer patients in whom neuropathic pain of lumbosacral plexus involvement in terminal pelvic cancer had been refractory to other therapies and those who already having urinary diversion procedures or colostomies with minimal risks of urinary or rectal incontinence. Clinical efficacy of the procedure was assessed by comparing patient pain ratings and narcotic usage pre- and post-SDR.

Results: Examination of the results indicated a significant reduction in pain ratings as well as a significant reduction in daily narcotic use. No adverse neurological effects were observed and no recurrence of pain of neoplastic lumbosacral plexopathy was noted. Conclusions: These findings provide corroborating clinical evidence for the effectiveness of selective dorsal root rhizotomy for the intractable pain of lumbosacral plexopathy in terminal pelvic cancer patients. 
Session Title: Flash Session - Stereotactic Technology

Session Time: Wednesday, May 29, 2013, 3:30 pm - 4:30 pm

Presentation Number: 140

Definition of a Stereotactic 3d Model of the Human Insula for Neurosurgical Approach (epilepsy and Stereotaxic Surgery) Afif M. AFIF, MD,PhD, Lyon-1 University, Lyon, France; Guillaume BECQ, Grenoble, France; Patrick MERTENS, MD,PhD, Lyon, France

Introduction: Design a method for 3D reconstruction of the insula, including its gyri and sulci, in AC-PC reference usable individually for imaging or for epilepsy and stereotactic surgery.

Methods: Morphometric study using 56 MRI of normal insular region. 26 male/30 female, 28 left/28 right hemispheres. Stage 1:

Reconstruction in AC-PC reference of the insula from 3D-T1-MRI slices $1 \mathrm{~mm}$ thick. Stage 2: Digitalization and superposition of data in 3D using PhotoStudio software (Photo Editing Software) system with PC as the center of coordinates. Stage 3: MATLAB software (Mathworks Inc.) was used to transform in color

values each pixel to obtain a color scale corresponding to the probability of insula sulci localization between $0 \%$ and $100 \%$.

Results: Demonstration of very significant correlations between the coordinates of the main insular structures (angles, sulci..) and the length of AC-PC. This close correlation allows to describe a method for 3D reconstruction of the insula on MRI slices that requires only the positions of Ac and PC and then the inter-commissural (AC-PC) length. This procedure defines an area containing insula with $100 \%$ probability.

Conclusions: 3D reconstruction of insula will be potentially useful for:

1- To improve localization of cortical areas, allowing to differentiate insular cortex from opercular cortex during stereoelectroencephalographic exploration of patients with epilepsy (SEEG) or in morphological and functional imaging.

2 - For microsurgical approach of Insula using Neuronavigation techniques.

3 - Identification of Insula during stereotactic surgery (SEEG, biopsy). 
Session Title: Flash Session - Stereotactic Technology

Session Time: Wednesday, May 29, 2013, 3:30 pm - 4:30 pm

Presentation Number: 183

Robotics in Neurosurgical Stereotactic Interventions: Oblique Intrainsular Electrodes Implanted of Patients with Epilepsy Afif M. AFIF, MD,PhD, Neurosurgery Department, Neurological Hospital, University Lyon 1, France, Lyon, France

Introduction: This study is to investigate: The feasibility, the safety and the utility of chronic depth electrodes stereotactically implanted by a robotic arm in the insular cortex of patients suffering from drug refractory focal epilepsy.

Methods: A total number of 32 electrodes in 29 patients (in Grenoble University Hospital) were successfully implanted within the insula. 220 contacts were available for insula recording. Electrode insertion was guided by a robotic arm (Neuromate, Renishaw mayfield, Switzerland) connected to the stereotactic frame and driven by stereotactic planning software. The targetting of the insula is planned on a pre-surgical T1-MRI. The fusion between the preoperative 3D MRI and the postoperative 3D CT scan enabled us to identify the contact location in three dimensions.

Results: No morbidity occurred during the surgical step and the chronic SEEG recording or stimulation procedure. Clinical responses have been identified in terms of gyral and sulcal anatomy. They were classified into: painful responses, sensitivomotor responses, speech disturbance, oropharengeal responses, auditory phenomena and neuro-vegetative phenomena.

Conclusions: The advantages of the oblique approach are:

1. The implantation of electrodes within the insula using robotic arm appears in our study to be safe.

2. This approach can explore all insular regions by avoidance of the sylvian vascular network.

3. This approach offers a better sampling of insular EEG activity (until 10 contacts/electrode) than that obtained by the classical lateral trans opercular approach (1 $1 \frac{1}{2}$ contacts/electrode).

4. This approach has allowed us to develop the first anatomo-functional organization scheme of the insular cortex according to its gyri and sulci. 
Session Title: Flash Session - Stereotactic Technology

Session Time: Wednesday, May 29, 2013, 3:30 pm - 4:30 pm

Presentation Number: 75

\section{Comparison Of Voltage And Current Driven Deep Brain Stimulation}

Sam Eljamel, MBBCh, MD, FRCSIre, FRCSEd, FRCS(SN), The University of Dundee, Dundee, United Kingdom; Andrew Brown, BMSc, Dundee, United Kingdom; Suzanne Sinclair, RCN, Dundee, United Kingdom

Introduction: DBS had gained acceptance in the treatment of $\mathrm{PD}$, dystonia and tremor and currently undergoing prospective evaluation in the fields of epilepsy, psychiatry and pain. As technology had evolved over the last decade from voltage based IPG technology to current driven technology, we converted 21 consecutive patients from voltage based to current driven devices and evaluated the clinical response.

Methods: Forty leads in 21 patients stimulated by voltage based IPGs were converted to constant current IPGs using pocket adaptors. 24 leads were in the STN, 4 in the GPI, and 12 were in the VIM. We administered questionnaires to patients before and after the switch over and evaluated IPG settings prospectively.

Results: The mean amplitude to achieve the best clinical response was $3.26 \mathrm{~V}$ compared to $2.73 \mathrm{~A}$ (p 0.05). The mean impedance before the switch over was $1312 \mathrm{Ohms}$ compared to $1342 \mathrm{Ohms}(\mathrm{p}<0.05)$. All patients felt that constant current DBS stimulation had controlled their symptoms smoother compared to voltage stimulation and they did not use their patient controllers to increase or decrease the amplitude of their IPGs

Conclusions: Constant current DBS settings provided smoother symptom control in DBS therapy with reduced amplitude requirement and potential battery saving. 
Session Title: Flash Session - Stereotactic Technology

Session Time: Wednesday, May 29, 2013, 3:30 pm - 4:30 pm

Presentation Number: 142

\section{Early Experience with MRI-directed Implantable Guide Tube Technique for Deep Brain Stimulation}

Christopher R. P. Lind, FRACS, University of Western Australia, Perth, Australia; Nova B. Thani, FRACS, Perth, Australia; Arul Bala, FRACS, Perth, Australia; Elaine Pang, Perth, Australia; Nathan Yap, Perth, Australia; Rick Stell, FRACP, Perth, Australia; Julian P. Rodrigues, FRACP, Perth, Australia; Megan Thorburn, Perth, Australia; Susan E. Walters, Perth, Australia; Simone Brockman, BSc, Fremantle, Australia; Sergio E. Starkstein, MD, PhD, FRANZCP, Fremantle, Australia

Introduction: The MRI-guided implantable guide tube technique (GT) of Gill is a completely stereotactic technique for deep brain stimulation (DBS).

Methods: The GT technique was adopted for the first time outside Bristol, UK with minor modifications. Accuracy and outcomes were assessed in a consecutive series of 36 patients undergoing GT DBS from April 2009 to December 2012.

Results: In the early part of this series we published three-dimensional inaccuracy of delivering a carbothane stylette and thus DBS lead to the imaging target to be $1.8 \mathrm{~mm}(95 \%$ CI: $1.5 \mathrm{~mm}, 2.1 \mathrm{~mm})$ and air entrainment median of $0.00 \mathrm{~mL}$ (range $0-2.52 \mathrm{~mL})$. Safe trajectories providing the surgeon with $97.5 \%$ confidence of avoiding hazards could always be planned. Twenty seven posterior subthalamic area (PSA), 1 anterior GPi, 1 STN, and 7 postero-ventral GPi cases have been performed. Twenty six PD, 5 tremor, 1 Tourette's, 4 dystonia cases were operated. In those with PD undergoing PSA DBS, unblinded MDS-UPDRS III improved from a mean of 48/22 without/with medications to 10 with both stimulation and medications at 6 months. There were associated marked improvements in quality ON-time in diaries and on standard quality of life measures with no decrement in neuropsychological performance or major psychiatric disorders. The smaller movement disorder groups improved well.

Conclusion: The GT technique has distinct advantages in an era of evolving stereotactic indications and brain targets: (1) it is optimized for general anesthesia during imaging and surgery facilitating accuracy and tolerability; (2) pull-out of DBS leads from the brain target is obviated by prevention of intracranial air entrainment; and (3) electrode positions can be safely and accurately documented without artifact using MRI. We have been able to achieve good clinical results without the patient being awake and without intra-operative neurological examination, stimulation or physiological recordings. 
Session Title: Flash Session - Stereotactic Technology

Session Time: Wednesday, May 29, 2013, 3:30 pm - 4:30 pm

Presentation Number: 111

\section{A Novel Recessed-step Catheter for Reflux-free Convection-enhanced Drug Delivery to the Brain}

Neil Barua, BMBS, BMedSci, MRCS, Frenchay Hospital, Bristol, United Kingdom; Thomas Gill, University of Bristol, United Kingdom; Max Woolley, PhD, Renishaw, United Kingdom; Alison Bienemann, PhD, University of Bristol, United Kingdom; Dave Johnson, PhD, Renishaw, United Kingdom; Stephen O'Sullivan, Renishaw, United Kingdom; Gavin Murray, Renishaw, United Kingdom; Catriona Fennelly, Renishaw, United Kingdom; Owen Lewis, Renishaw, United Kingdom; Charlie Irving, Renishaw, United Kingdom; Marcella Wyatt, University of Bristol, United Kingdom; Steven Gill, FRCS, MS, Frenchay Hospital, Bristol, United Kingdom

Introduction: The optimisation of convection-enhanced drug delivery (CED) to the brain is fundamentally reliant on minimising drug reflux, in order to maximise the volume of distribution and minimise the risk of off-target side-effects. The aim of this study was to evaluate the performance of a novel reflux-resistant CED catheter incorporating a recessed-step and to compare its performance to previously described stepped catheters.

Methods: The in vitro performance of the recessed-step catheter was compared to a conventional "one-step" catheter with a single transition in outer diameter (OD) at the catheter tip, and a "two-step" design comprising two distal transitions in OD. All catheters had a $3 \mathrm{~mm}$ length of fused silica at the distal tip. The volumes of distribution and reflux were compared by performing infusions of Trypan blue into agarose gels. The in vivo performance of the recessed-step catheter was then analysed in a large animal model by performing bilateral intraputaminal infusions of $0.2 \%$ Gadolinium-DTPA in a Large White/Landrace pig.

Results: Comparison of the volumes of distribution (Vd)and infusion (Vi) demonstrated the recessed-step catheter to have a significantly higher $\mathrm{Vd} / \mathrm{Vi}$ than the one-step and two-step catheters $(\mathrm{p}=0.0001$, one-way ANOVA). No reflux was detected until more than $100 \mu \mathrm{l}$ had been delivered via the recessed-step catheter, whilst reflux was detected after infusion of only $25 \mu 1$ via the 2 nonrecessed catheters. A total volume of $587.5 \mu \mathrm{l}$ was infused into both putamen of a Large White/Landrace pig at a maximum infusion rate of $5 \mu \mathrm{l} / \mathrm{min}$. There was no evidence of reflux of contrast beyond the superior border of the putamen in either infusion.

Conclusions: The novel recessed-step catheter described in this study shows significant potential for the achievement of predictable high volume, high flow rate infusions via multiple catheters in an acute and sub-chronic setting, whilst minimising the risk of reflux. 
Session Title: Flash Session - Stereotactic Technology

Session Time: Wednesday, May 29, 2013, 3:30 pm - 4:30 pm

Presentation Number: 364

The Yield And Safety Of Robotic-assisted Stereotactic Biopsy Versus Frameless Stereotactic Biopsy

Tariq Aljared, MD, Montreal Neurological Hospital, Montreal, QC, Canada; Jeffery Hall, MD, Montreal, QC, Canada; Andre Olivier, MD,PhD., Montreal, QC, Canada

Introduction: Robotic-assisted surgery is an evolving technique in neurosurgery. The literature focuses on its novelty. Our study aims to compare it to a standard technique.

Methods: Retrospective analysis of stereotactic biopsies in Montreal Neurological Hospital. All robotic-assisted stereotactic biopsies since its introduction (2011) and all frameless stereotactic biopsies since 2008 were included. We limited our study to the 2 senior authors who are familiar with both techniques.

Results: 10 patients underwent 11 robotic-assisted stereotactic biopsies and 16 patients underwent 17 frameless stereotactic biopsies. The diagnostic yield among the 2 groups was $8 / 11$ and 14/17 diagnostic procedures, respectively (p-value 0.65 ). Post-operative imaging were obtained in 8 patients with robotic-assisted surgery and 13 patients of frameless stereotactic biopsy. There was only 1 biopsy-related hemorrhagic changes in robotic cases and 4 in frameless cases (p-value 0.61 ). None of the hemorrhagic changes were clinically significant. In contrast to frameless biopsies, All robotic-assisted biopsies were deep-seated and eloquent-related lesions.

Conclusions: The yield of robotic-assisted and frameless stereotactic biopsies is comparable. In terms of safety, it seems that there is a trend toward less biopsy-related hemorrhage with robotics technique. This impression of safety, has translated into confidence in establishing diagnosis in critically located lesions with robotic techniques. 


\section{Presentation Number: 344}

\section{Simultaneous 32-contact Local Field Potential Recordings In The Parkinsonian STN}

Rick Schuurman, Academic Medical Center Amsterdam, Amsterdam, Netherlands; Rens Verhagen, MSc, Amsterdam, Netherlands; Fiorella Contarino, MD, PhD, Amsterdam, Netherlands; Rob de Bie, Amsterdam, Netherlands; Gijs van Elswijk, Sapiens, Eindhoven, Netherlands; Hubert Martens, Sapiens, Eindhoven, Netherlands; Pepijn van den Munckhof, MD, PhD, Amsterdam, Netherlands; Lo Bour, Amsterdam, Netherlands

Introduction: The optimal mode of stimulation with a new 32 contact DBS lead, which is capable of steering stimulation, could be guided by the information obtained from electrophysiological signals recorded from the 32 contact points. Spatial and temporal content of the LFPs, related to the patient's symptomatology could be used to determine how to direct the current delivered through a selection of the contact points. The objective of this study is to perform simultaneous 32 channel local field potential recordings (LFPs) across the human STN for the determination of the distribution of oscillatory power in patients with Parkinson's disease. Methods: Ten patients with Parkinson's disease are included in the study. After micro electrode recordings (MER) the new 32-contact electrode is temporarily inserted during surgery. MER performed at different depths during surgery are compared with the 32 channel LFPs along a length of $7 \mathrm{~mm}$.

Results: The distribution of spectral information and coherency across the electrode array shows a clear relationship with the morphology and the abnormal oscillatory behavior of the Parkinsonian STN.

Conclusions: Through 32 smal contacts of a new DBS lead it is possible to perform simultaneous LFP recordings across the entire STN without moving the electrode, providing spatial information about disease-related electrical activity of the STN. This can potentially be of benefit in predicting how to steer the current towards the sensomotoric part and avoid stimulation of brain structures that induce adverse effects. 
Session Title: Flash Session - Stereotactic Technology

Session Time: Wednesday, May 29, 2013, 3:30 pm - 4:30 pm

\section{Presentation Number: 416}

\section{Use of a Novel, Rapidly Generated Custom Platform for Stereotactic DBS Implantation}

Jason Agran, MD, PhD, Vanderbilt University, Nashville, TN; Joseph Neimat, MD MS, Vanderbilt University, Nashville, TN; Ramya Balachandran, PhD, Nashville, TN; Srivatsan Pallavaram, PhD, Nashville, TN; Gregoire S. Blanchon, PhD, Nashville, TN; Michael J. Fitzpatrick, $\mathrm{PhD}$, Nashville, TN

Introduction: Traditional stereotacic frames provide significant targeting flexibility but are cumbersome and may be uncomfortable for patients. More recent targeting platforms have been designed to be more comfortable and to improve procedural efficiency. The process of creating and shipping such platforms takes several days and may inconvenience patients. Our goal was to employ a rigid, rapidly customizable, microstereotactic frame called a "Microtable" that achieves submillimetric accuracy for the implantation of stereotactic electrodes in Deep Brain Stimulation (DBS) surgery.

Methods: The Microtable system consists of a computer-numerical-control (CNC) machine-milled Lexan tabletop that mounts on surgically implanted bone markers. Unique to the Microtable is its capability for rapid generation, resulting from the simple expedient of drilling a set of parallel holes through a single planar tabletop, which then mounts to a set of standardized table legs. The position and depth of these holes is sufficient to specify any required stereotactic trajectory. The technology was tested for stability and accuracy on phantoms and on a cadaveric specimen. It has now been implemented in a single DBS implantation, which is reported here.

Results: The Microtable was successfully used for the implantation of a single VIM DBS electrode. The procedure was performed less than $24 \mathrm{hrs}$ after bone marker implantation. A single trajectory was performed without need for targeting adjustments. Time from incision to electrode implantation was $47 \mathrm{~min}$. Post-surgical accuracy was measured as $1.72 \mathrm{~mm}$, which compares favorably with reported accuracy of the Starfix platform of $1.99 \mathrm{~mm}$. The patient exhibited significant relief of tremor with intraoperative stimulation. Conclusions: The Microtable is a viable alternative to other stereotactic methods, incorporating the advantages of customized platforms but improving on surgical process flow. The device is also distinguished by its relative cost effectiveness. It is the combination of speed and cost that differentiates the Microtable from others clinically available alternatives. 
Presentation Number: 221

\section{Identification Of Blood Vessels With Micro Doppler Ultrasound Songraphy In Stereotactic Functional Neurosurgery Via Microelectrode Guide Tubes- A Prototype of High Value!}

Wilhelm E. Eisner, Prof.,MD, Medical University Innsbruck, Austria, Innsbruck, Austria

Introduction: In 1982 transcranial Doppler ultrasound sonography got introduced to the neurosurgical / neurological community by Aaslid R. Soon transcranial doppler sonography became the bedside method in monitoring of patients with subarachnoidal hemorrhages and vasospasm. Small ultrasound probes with diameters around $2.5 \mathrm{~mm}$ were used around 1990 in stereotactic and vascular neurosurgery. We and few others are utilizing intraoperative doppler sonography in stereotactic procedures like diagnostic biopsies. The range in diameter of the probes varies from $1.3 \mathrm{~mm}$ up to $2.5 \mathrm{~mm}$ or bigger. Considering certain rules stereotactic surgery is very safe. One of these rules is: never penetrate the brain by a sharp edged instrument like an intravenous needle - it will cause a bleeding. All tips of stereotactic instruments are rounded like dolphin noses. Microrecording electrodes are able to carry a higher risk of bleeding because of its sharp $(2-3 \mu \mathrm{m})$ electrode tip. Multichannel microrecording increases statiscically the risk of intracerebral bleeding.

Methods: We developed in 2000 the prototype of an intraoperative doppler ultrasound probe with a diameter less than $0.8 \mathrm{~mm}$. The probe is fitting into the microelectrode guiding tubes and is able to check the trajectory for blood vessels unseen in imaging.

Results: In the last 12 years not a single bleeding occurred in our patients. We will demonstrate the instrument and its usage. The development and utilization of that instrument is supported by our surgical data.

Conclusions: In 30 years doppler ultrasound examinations has proven its value for surgery. 
Presentation Number: 66

\section{Supine Position reduces Brain Shift during Stereotactic Neurosurgery}

Yasushi Miyagi, MD, PhD, Kaizuka Hospital, Fukuoka, Japan

Introduction: Brain shift can be one of the significant error factors for an accuracy of stereotactic neurosurgery. We investigated an effect of supine position on the brain shift during bilateral stereotactic lead implantation of deep brain stimulator.

Methods: Twenty-three patients $(\mathrm{n}=23 ; \mathrm{M} / \mathrm{F}=12 / 11$; age $=59.1 \pm 12.6$ years) underwent the contemporaneous implantation of bilateral DBS electrodes at 4 globus pallidus internus and 19 subthalamic nuclei. The surgeries were performed in supine position with magnetic resonance (MR) imaging-guided and microelectrode-guided methods. The outflow of CSF was prevented by fibrin glue onto the arachnoid around the electrodes or cannulas. Brain shift was assessed as changes in the 3D coordinates of the anterior and posterior commissures (AC and PC) with MR imaging before and immediately after the implantation surgery. The data were compared with those obtained by the prior surgical technique $\left(\mathrm{n}=10\right.$, twist-drill surgery in semi-sitting position, $36.2 \pm 4.3^{\circ}$, Miyagi et al. J Neurosurg 107: 989, 2007).

Results: In the supine position, the shift of AC was as follows (mm, mean $\pm \mathrm{SD}$ ): $\mathrm{x}=0.03 \pm 0.18, \mathrm{y}=0.53 \pm 0.29, \mathrm{z}=0.13 \pm 0.21$ and the shift of PC was as follows: $\mathrm{x}=0.02 \pm 0.14, \mathrm{y}=0.24 \pm 0.26, \mathrm{z}=0.10 \pm 0.18$. The changes in ACPC distance were $0.28 \pm 0.31 \mathrm{~mm}$. Except for the $\mathrm{z}$ coordinate of $\mathrm{AC}$, all the coordinates of $\mathrm{AC}$ and $\mathrm{PC}$ were significantly smaller in the supine position than in the semisitting position (Student's t-test).

Conclusions: The extents of brain shift and brain deformation (shortening of AC-PC distance) were significantly smaller in the supine position. Supine position may minimize the negative intracranial pressure and prevents the intracranial air invasion and brain shift. 
Session Title: Flash Session - Stereotactic Technology

Session Time: Wednesday, May 29, 2013, 3:30 pm - 4:30 pm

Presentation Number: 213

Impact Of Parameters Of Radiofrequency Coagulation On Volume Of Stereotactic Lesion In Pallidotomy And Thalamotomy Hidehiro Hirabayashi, National Hospital Organization Nara Medical Center, Nara, Japan; Marwan I. Hariz, MD,PhD, London, United Kingdom; Patric Blomstedt, MD,PhD, Umeå, Sweden; Karin Wårdell, PhD, Linköping, Sweden

Introduction: One of the many reasons why lesional surgery for movement disorders has been more or less abandoned may have been the difficulty in predicting the shape and size of the stereotactic radiofrequency (RF) lesion. To analyse the contribution of various RF coagulation parameters towards the volume of pallidotomies and thalamotomies.

Methods: The relationship between temperature of coagulation, length of coagulated area, and duration of coagulation on one hand, and lesion volume on the other hand, was retrospectively evaluated. Lesion diameters were measured on stereotactic thin slice CT and MRI scans, and volumes of lesions were calculated concerning 36 pallidotomies and 14 thalamotomies in 46 patients who were operated using the same RF generator and same RF electrode.

Results: The coagulation temperature, length of coagulated area, and duration of coagulation were all correlated to the lesion volume. However, for a given length of coagulated area, the lesion's size was most strongly influenced by the temperature. Despite this clear correlation, and the relatively homogenous coagulation parameters, the lesions' volumes were markedly scattered.

Conclusions: The volume of the stereotactic RF lesions could be correlated to the coagulation parameters, especially the temperature, at a group level, but could not be predicted in individual patients based solely on the RF

coagulation parameters. 
Presentation Number: 205

Neuronal Activity In The Prefrontal-cortex Is Reduced In Rats Selectively Bred For Disturbed Sensorimotor Gating Mesbah Alam, PhD, Hannover Medical School, Hannover, Germany; Svilen Angelov, Hannover, Germany; Maike Getschmann, Hannover, Germany; Joachim Kurt Krauss, MD,PhD, Hannover, Germany; Kerstin Schwabe, PhD, Hannover, Germany

Introduction: Rats selectively bred for deficient prepulse inhibition (PPI), an operant measure of sensorimotor gating, may be used to study the pathophysiological mechanisms and therapeutic strategies for neuropsychiatric disorders with abnormalities in information processing, such as schizophrenia and Tourette's syndrome. The medial prefrontal cortex (mPFC) and the nucleus accumbens (NAC) have been shown to be involved in the regulation of PPI. Additionally, lesions of the entopeduncular nucleus (EPN) alleviated the PPI in rats with low PPI. We here examined the neuronal activity in the MPFC, NAC and EPN, since these areas are possibly involved in the pathomechanisms of prepulse inhibition (PPI) of the acoustic startle reaction.

Methods: Male rats with breeding-induced high and low expression of PPI ( $\mathrm{n}=6$, each) were anesthetized with urethane $(1.2 \mathrm{mg} / \mathrm{kg})$. Extracellular single unit activity and local field potentials were recorded in the mPFC, NAC, and EPN for ten minutes each.

Results: In PPI low rats, the discharge rate, measures of irregularity and the burst activity were significantly reduced compared to the measures in PPI high rats $(\mathrm{p}<0.05)$, while analysis of the neuronal activity in the NAC and EPN showed no difference between groups. Additionally, the oscillatory theta band activity $(4-8 \mathrm{~Hz})$ was enhanced and the beta band activity $(13-30 \mathrm{~Hz}) \mathrm{was}$ reduced in all regions.

Conclusions: Reduced neuronal activity in the MPFC of PPI low rats may be one of the pathophysiological mechanisms leading to reduced sensorimotor gating. Interestingly, enhanced theta band activity has been found also in patients with Tourette's syndrome and dystonia, i.e., disorders, that are accompanied by sensorimotor gating deficits. 
Presentation Number: 390

Validating Diffusion Tensor Imaging of the Thalamus with Intraoperative Mircroelectrode Recording

Hiroki Toda, MD,PhD, Tazuke Kofukai Medical Research Institute and Kitano Hospital, Osaka, Japan; Nobukatsu Sawamoto, MD,PhD, Kyoto, Japan; Ryosuke Okumura, MD,PhD, Osaka, Japan; Namiko Nishida, MD, PhD, Osaka, Japan; Hidemoto Saiki, MD,PhD, Osaka, Japan; Sadayuki Matsumoto, MD,PhD, Osaka, Japan; Koichi Iwasaki, MD,PhD, Osaka, Japan

Introduction: Identification of the anteroposterior borders of the ventral thalamic nuclei is important in thalamic stimulation surgery. We have reviewed magnetic resonance imaging data in five patients with tremor who underwent thalamic stimulation.

Methods: We have analyzed the radiographical boundaries of the thalamic ventral nuclei using a deterministic and a probabilistic tractography algorithms with 3-Tesla diffusion imaging data. These tractography results are reviewed with structural magnetic resonance images and intraoperative microelectrode recording data of the thalamic ventral nuclei.

Results: The length of the motor-somatosensory boundaries was 5.5 to $8.5 \mathrm{~mm}$. The highest connectivity of hand motor area was located at 12 to $16 \mathrm{~mm}$ lateral from the midline and 6 to $10 \mathrm{~mm}$ anterior to the posterior commissure on the anterior and posterior commissural plane. The distance from the pyramidal tract to the implanted electrode was 1.8 to $3.3 \mathrm{~mm}$.

Conclusion Diffusion tensor imaging is useful for preoperative estimation of anteroposterior border of the thalamic ventral nuclei, which support safe and effective surgical planning of the thalamic stimulation. 
Session Title: Flash Session - Stereotactic Technology

Session Time: Wednesday, May 29, 2013, 3:30 pm - 4:30 pm

Presentation Number: 263

\section{Diagnostic Yield of ${ }^{1}$ H-MRS-supported Stereotactic Brain Biopsy: Overview of Published Results}

Mikhail F. Chernov, MD,DMedSci, Tokyo Women's Medical University, Tokyo, Japan; Yoshihiro Muragaki, MD, PhD, Tokyo, Japan; Hiroshi Iseki, MD, PhD, Tokyo, Japan; Takaomi Taira, MD. PhD, Tokyo, Japan; Yoshikazu Okada, MD, PhD, Tokyo, Japan

Introduction: ${ }^{1} \mathrm{H}$-MRS-support during target selection for stereotactic biopsy of parenchymal brain lesions may potentially improve effectiveness of tissue sampling, which may be particularly important in cases of gliomas. The objective of the present study was evaluation of the efficacy of this technique based on the published results.

Methods: The main criteria applied for selection of references during literature search was unequivocal statement that spectroscopic data were used for choice of the target for stereotactic brain biopsy. Thirteen reports published from 2001 to 2012 were identified. Four studies originated from the same center, therefore only the latest one was taken into consideration. It limited the total number of references to 10. The studies were characterized as Case Report (1), Case Series (7), Case-Control Study (1), Prospective Randomized Trial (1).

Results: The total number of reported patients was 482 with a range in a separate series from 2 to 296 (median 15). Proportion of parenchymal brain tumors varied in separate series from 0.77 to 1.00 . Target selection was most frequently based on the highest value of Cho/NAA ratio ( 7 series). Just in 2 cases tissue sampling was considered non-diagnostic. Therefore, an overall diagnostic yield of ${ }^{1}$ H-MRS-supported brain biopsy was $99.6 \%$ (range in separate series from $98.4 \%$ to $100 \%$ ). Direct comparison of MRI-guided and MRS-supported techniques was done in 2 studies, and both reports showed the advantages of the latter. The rate of clinically significant complications did not exceed $1 \%$.

Conclusions: Use of spectroscopic information for target selection for stereotactic biopsy of parenchymal brain lesions may have significant positive impact on the diagnostic yield of the procedure. Prospective controlled studies are necessary to establish its diagnostic accuracy and optimal selection of the metabolic target. 


\section{Deep Brain Stimulation Of The Fornix And Entorhinal Cortex In An Experimental Model Of Memory Impairment}

Ali Jahanshahi, Maastricht University, Maastricht, Netherlands; Sarah Hescham, Maastricht, Netherlands; Jacobus van Overbeeke, Maastricht, Netherlands; Yasin Temel, Maastricht, Netherlands

Introduction: Deep brain stimulation (DBS) is a surgical treatment involving the implantation of electrodes, which give electrical impulses to specific parts of the brain. Recently, DBS in the region of the fornix has been applied in Alzheimer's disease with the purpose of improving or reducing the progression of memory loss. When structures of the memory circuitry are stimulated, DBS is thought to enhance neural activity and thus improves performance on memory tasks.

Methods: In this study, we implanted bilateral electrodes at the site of the fornix, dorsal and ventral hippocampus, and entorhinal cortex in order to detect which stimulation parameters provide beneficial effects in spatial memory. Rats were tested in the Object Location Task with the following conditions: (i) with attachment of stimulation cable (off stimulation), and (ii) with DBS at various amplitudes (50 $\mu \mathrm{A}, 100 \mu \mathrm{A}$ and $200 \mu \mathrm{A}), 100 \mu$ s pulse width and $100 \mathrm{~Hz}$ or $10 \mathrm{~Hz}$ stimulation frequency. Intraperitoneal scopolamine injections 30 minutes before the first trial were given to imitate memory impairment.

Results: DBS of the forniceal region and entorhinal cortex reversed the scopolamine effects in high current densities and showed superior memory performance when compared to hippocampal stimulated and sham rats. Hippocampal stimulation showed no beneficial effect on memory impairments. DBS of the forniceal region and entorhinal cortex, with the most efficient stimulation parameter, had no effect on anxiety-like behavior in the Open Field and Elevated Zero Maze, suggesting no potential side effects regarding anxiety levels or general motor activity. Finally, immunohistochemical analysis revealed elevated expression of c-Fos levels in DBS treated rats in regions of interest.

Conclusion: DBS of the forniceal region and the entorhinal cortex with specific stimulation parameters enhances the activity of the memory circuit and improves memory impairment in an experimental model. 


\section{Presentation Number: 90}

Results Of Neuromodulation Of The Posterolateral Hypothalamic Region In Cluster Headache. Are We On The Right Place? Fernando Seijo, MD, Hospital Universitario Central de Asturias, Oviedo, Spain

Introduction: Cluster Chronic Headache $(\mathrm{CCH})$ is included in the group of trigeminal autonomic cephalgias and its clinical picture is characterized by very intense trigeminally distributed pain and autonomic facial dysfunction, with $10-20 \%$ of patients not responding to any type of medication. CH pathophysiology has not been sufficiently established yet but vascular and central origin are the most widely accepted theories. Based on the latter, 64 patients with drug-resistant $\mathrm{CCH}$ underwent posteroinferior hypothalamus deep brain stimulation (DBS) worldwide. Overall success rate was $64 \%$ with mean follow-up of 1-6 years. Our objective was to study the efficacy of posterolateral hypothalamic neuromodulation in $\mathrm{CCH}$ patients.

Methods: 8 patients ( 7 male, 1 female, mean age $47.50 \pm 1.95$ [SD] years) with an average number of 47 attacks/week underwent hypothalamic DBS. Stereotactic coordinates were $4 \mathrm{~mm}$ from the third ventricle wall, $2 \mathrm{~mm}$ from behind the mid-intercommissural point and $5 \mathrm{~mm}$ from under the intercommissural line. All patients complied with selection criteria and signed the Hospital Ethics Committee's informed consent.

Results: Mean stereotactic coordinates of the effective active contact were $4.85 \pm 0.69 \mathrm{~mm}$ [SD] from the third ventricle wall, $1.38 \pm$ $0.91 \mathrm{~mm}$ [SD] from behind the mid-intercommissural point and $4.18 \pm 1.00 \mathrm{~mm}$ [SD] below the intercommissural line. The follow-up time period was $26.75 \pm 24.88$ [SD] months. Average number of attacks post-surgery was 7, with 3 patients free of pain.

Conclusions: Posterolateral Hypothalamic DBS is a safe and effective therapy for the management of CCH patients. The stimulation field has a radius of approximately $3 \mathrm{~mm}$, covering the lateral hypothalamic area, fasciculus mammillo-tegmentalis, fasciculus mammillo-thalamicus and fasciculus medialis telencephali.

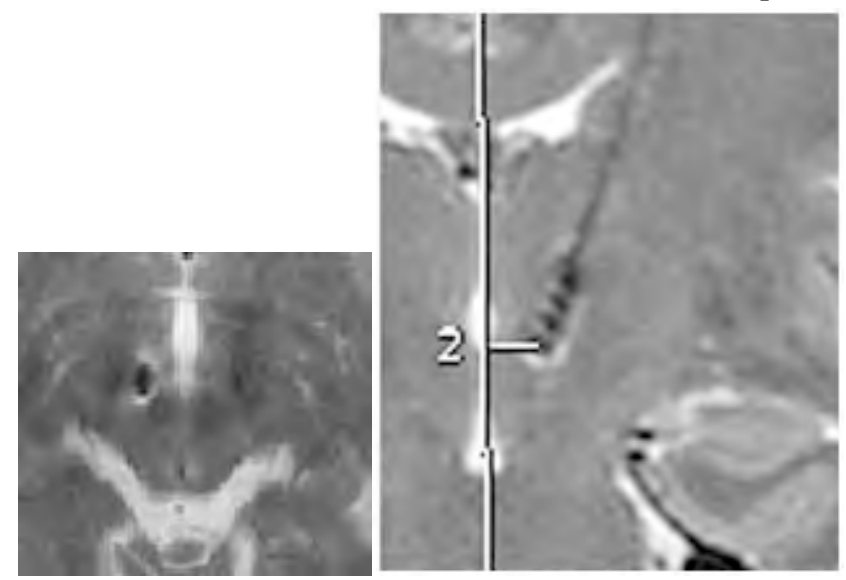


Presentation Number: 206

\section{An Exploratory Pilot Study of Intra-Thecal Spinal Cord Stimulation (IT-SCS): Implications for the future management of chronic pain.}

Nandan Lad, MD, PhD, Duke University Medical Center, Durham, NC

Introduction: Treatment of chronic back and leg pain with spinal cord stimulation (SCS) remains a significant clinical challenge. Only $60-75 \%$ of qualified patients receive permanent SCS implantation, with only $50 \%$ of these achieving long-term pain control. The shunting effect of the cerebrospinal fluid (CSF) within the Intra-Thecal (IT) space is considered the most important contributing factor to these sub-optimal outcomes. The primary objective of this early pilot study was to determine the safety and feasibility of IT-SCS via direct dorsal column stimulation and its ability to localize pain relief and paresthesia coverage.

Methods: Design: Prospective, controlled exploratory study comparing IT-SCS to epidural SCS.

Subjects: Patients with medically intractable pain who a) have post-laminectomy pain syndrome; b) previously had successful SCS, but became refractory; c) lacked adequate coverage during a prior SCS trial; d) were refractory to all other treatments and candidates for intrathecal catheter/pump. Computational modeling was performed examining various lead locations and field of coverage.

Results: During lead implantation, IT-SCS demonstrated robust stimulation and paresthesia coverage of low back alone, lower extremity alone and specific dermatomal distributions. An $\sim 10 x$ reduction in amplitude requirement compared to epidural SCS stimulation demonstrates the in vivo effect of CSF shunting. Modeling studies suggest that there is a steep gradient of stimulation effects on neural tissue as a function of distance, with $>95 \%$ of potentially targetable dorsal column fibers unable to be activated by standard epidural stimulators.

Conclusions: In this exploratory study, IT-SCS is able to produce focal coverage of low back pain without radicular stimulation at $1 / 10$ th the voltage, raising the possibility for dramatic increase in battery life and time between recharging intervals. Further studies are needed to examine the potential of IT-SCS to address the large number of patients currently excluded from neuromodulation approaches and with presently limited treatment options. 


\section{Presentation Number: 137}

\section{In Search Of Neurobiological Correlates To Depression: Electrical Stimulation Of The Amygdala}

Kelly R. Bijanki, Ph.D., University of Iowa Carver College of Medicine, Iowa City, IA; Christopher Kovach, Ph.D., Iowa City, IA; Laurie M. McCormick, M.D., Iowa City, IA; Hiroto Kawasaki, M.D., Iowa City, IA; John Wemmie, M.D., Ph.D., Iowa City, IA; Brian Dlouhy, M.D., Iowa City, IA; Matthew A. Howard, M.D., Iowa City, IA

Introduction: The amygdala is considered to play an important role in emotional regulation. The current study sought to further examine the role of the amygdala in emotional regulation by making use of a unique neurosurgical opportunity for direct electrical stimulation of the structure using an implanted depth electrode in a patient with epilepsy. The experiment aimed to examine transient changes in emotionality (self-rating of current mood) and affective bias during amygdala stimulation. Affective bias is a phenomenon wherein depressed patients interpret ambiguous or positive events as relatively negative. This phenomenon is especially pronounced in the rating of emotional facial expressions, a process with known amygdala involvement.

Methods: The participant rated his mood and the emotion expressed in an array of faces under two conditions; first under baseline conditions and then during intermittent stimulation $(5 \mathrm{sec})$ of the right amygdala at $50 \mathrm{~Hz}, 15 \mathrm{~V}$, with a pulse width of 200 microseconds. The facial expression stimuli included six identities (three men and three women), expressing overt and subtle happiness and sadness, as well as neutral expressions (Figure 1).

Results:On average, during stimulation, measures of the patient's mood improved by $7.8 \%$. In addition, he rated the facial expressions in the array as significantly more positive (Figure 2). Furthermore, an effect of emotional intensity was observed, where the more overt emotional faces were rated more positively than the subtle faces.

Conclusion: The current case is the first report of direct electrical stimulation to the amygdala producing elevations in mood and positive affective bias when viewing emotional facial expressions. These findings bear replication in a larger sample. These findings imply complex electrophysiological properties of the amygdala, and suggest that systematic examination of the effects of frequency, voltage, pulsewidth, and timing (intermittent vs. chronic stimulation) will be critical avenues for future study.
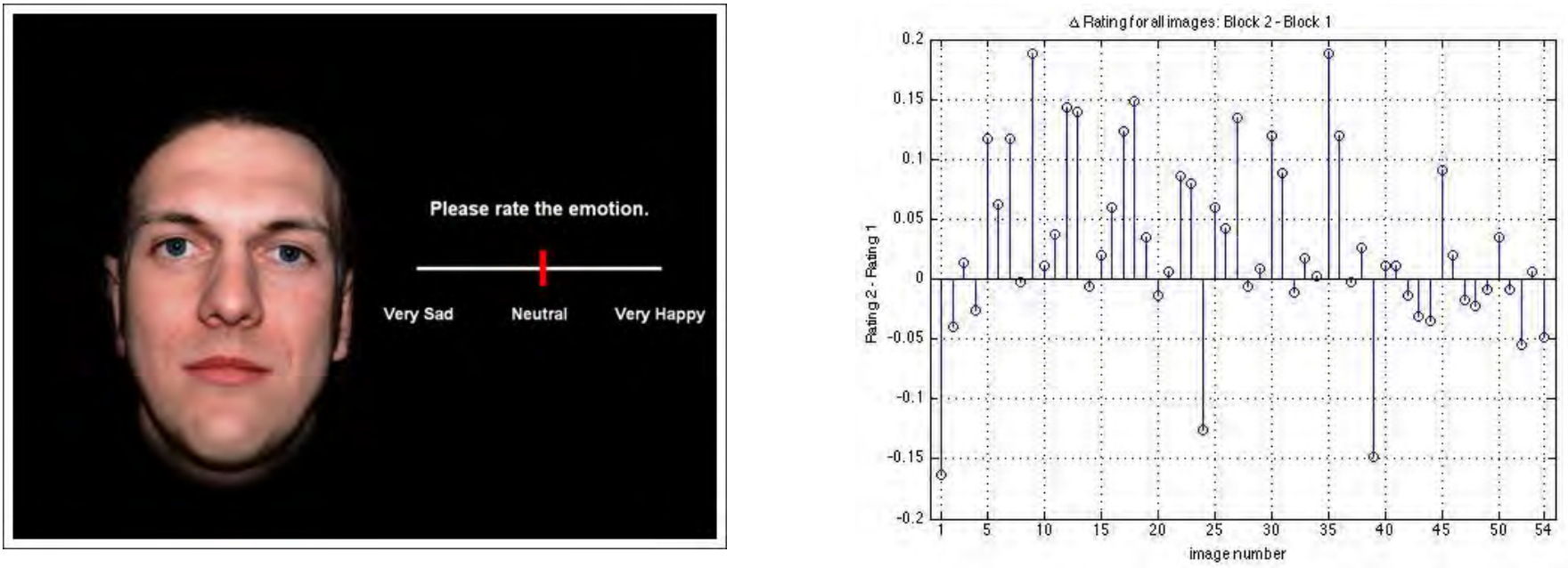


\section{Presentation Number: 13}

\section{Deep Brain Stimulation Eliminates Tic-related Neural Activity Via Interlocking With Stimulus Pulses}

Kevin W. McCairn, BSc PhD, Kyoto University-Primate Research Institute, Aichi, Japan; Atsushi Iriki, DDS, PhD, Wako-shi, Japan; Masaki Isoda, MD, PhD, Kansai, Japan

Introduction: A recent neurosurgical intervention that has shown potential for treating basal ganglia (BG) mediated motor tics, involves continuous high-frequency deep brain stimulation (HF-DBS) targeted to the output nucleus of the BG, the globus pallidus internus (GPi)

Methods: This study utilized a nonhuman primate model of BG-meditated motor tics and investigated the short-term neuronal mechanism that might underlie the beneficial effects of GPi-HF-DBS. In parallel with behavioral tic expressions, phasic alterations of neuronal activity emerged in the pallidum. We then delivered HF-DBS in the GPi in such a way that on-and off-stimulation conditions alternated every $30 \mathrm{~s}$.

Results: Analysis of electromyographic (EMG) records showed that during on-stimulation, there was a significant reduction in ticrelated EMG amplitude. Analysis of pallidal activity showed that GPi-HF-DBS induced both sustained and transient patterns of excitation and inhibition in both segments of the GP. Population-scale firing rates were initially raised relative to baseline, but were not significantly different by the time stimulation ceased. The modulation of behavior and neuronal firing rates was associated with the elimination of tic-related phasic activity in pallidal cells (Fig. 1). Examination of short-latency responses showed that firing rate changes were strongly associated with interlocking of the cells' activity with the HF-DBS pulse. This temporal interlocking often induced multiphasic changes of firing rates in individual cells that were dynamically changed across the stimulation period (Fig.2). Conclusions: These results support clinical studies that have reported success in treating motor tics with GPi-HF-DBS, and demonstrate that the underlying mechanism is elimination of tic-related activity through temporal interlocking with the stimulation pulse.
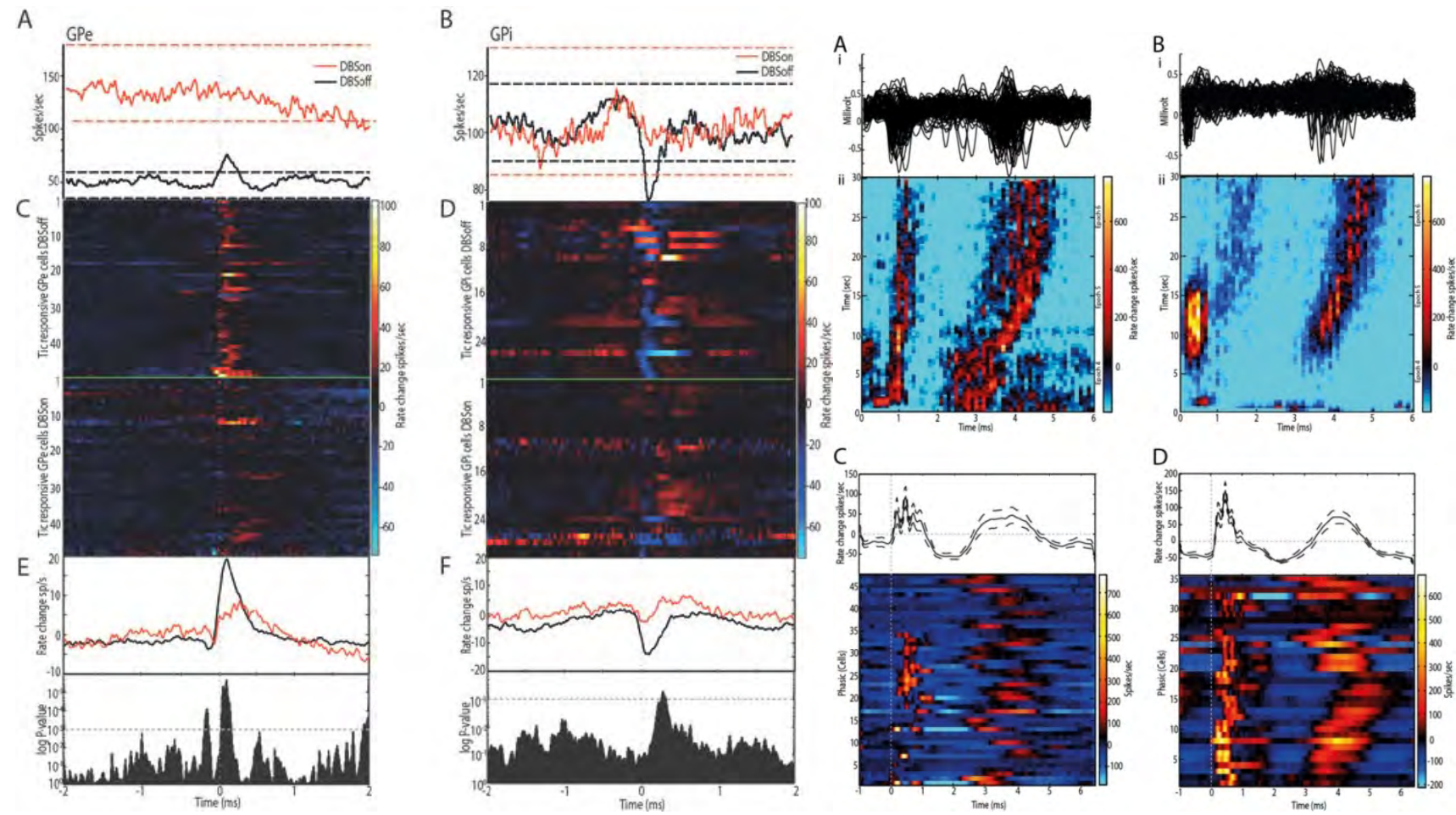


\section{Presentation Number: 94}

\section{Augmentation Of Perilesional Plasticity By Chronic Stimulation Of The Dentatothalamocortical Pathway In An Animal Model}

Andre Machado, MD, PhD, Cleveland Clinic, Cleveland, OH; Jessica Cooperrider, Cleveland, OH; Truong HaVan, PhD, Cleveland, $\mathrm{OH}$; Hyun Joo Park, PhD, Cleveland, OH; Zhihong Chengabigabi, PhD, Cleveland, OH; John Gale, $\mathrm{PhD}, \mathrm{Cleveland,} \mathrm{OH}$

Introduction: There is a pressing need for novel therapies to enhance post-stroke rehabilitation.

Methods: Long-Evans rats were pre-trained in the pasta-matrix task. Rats then underwent ischemia of the dominant motor cortex by intracortical endothelin injection and contralateral macroelectrode implantation aimed at the lateral cerebellar nucleus. After one week of evaluation, animals were assigned to either no stimulation or stimulation with regular or bursting patterns. All animals received the same physical training. Stroke volumes were quantified and electrode location verified. The perilesional cortex was evaluated with $3 \mathrm{D}$ electron microscopy.

Results: 31 animals completed the experiment. All electrodes were in the targeted region. There was no difference in pasta-task performance between groups prior to stroke. All groups had a significant deficit in the pasta matrix task following stroke ( $p<0.05$ ) and there was no difference in post-stroke performance between groups prior to stimulation onset. The SHAM (no stimulation) group did not recover over time. There was no significant improvement in performance compared to post-stroke baseline in any of the five weeks observed. Animals in the bursting stimulation (NONISOSTIM) group showed a significant improvement over their post-stroke baseline performance at every week of stimulation $(\mathrm{p}<0.05)$ while animals in the regular stimulation (ISOSTIM) group (30 Hz stimulation) showed a significant improvement from weeks $2-5$ of stimulation $(\mathrm{p}<0.05)$. Figure 1 summarizes the results. Evaluation of the perilesional cortex with 3D electron microscopy showed that animals in either stimulation group had 120.5 (+/- 32.9$)$ and 109.1 (+/- 22.4) synapses per 125 micron cube while those in the SHAM group had 41.8 (+/- 18.7) synapses. One-way ANOVA with Bonferroni post-hoc $\mathrm{p}<0.05$ (figure 2)

Discussion: The results indicate that chronic stimulation of the dentatothalamocortical pathway can enhance recovery of motor function and perilesional synaptic density. This approach has promising translational potential.
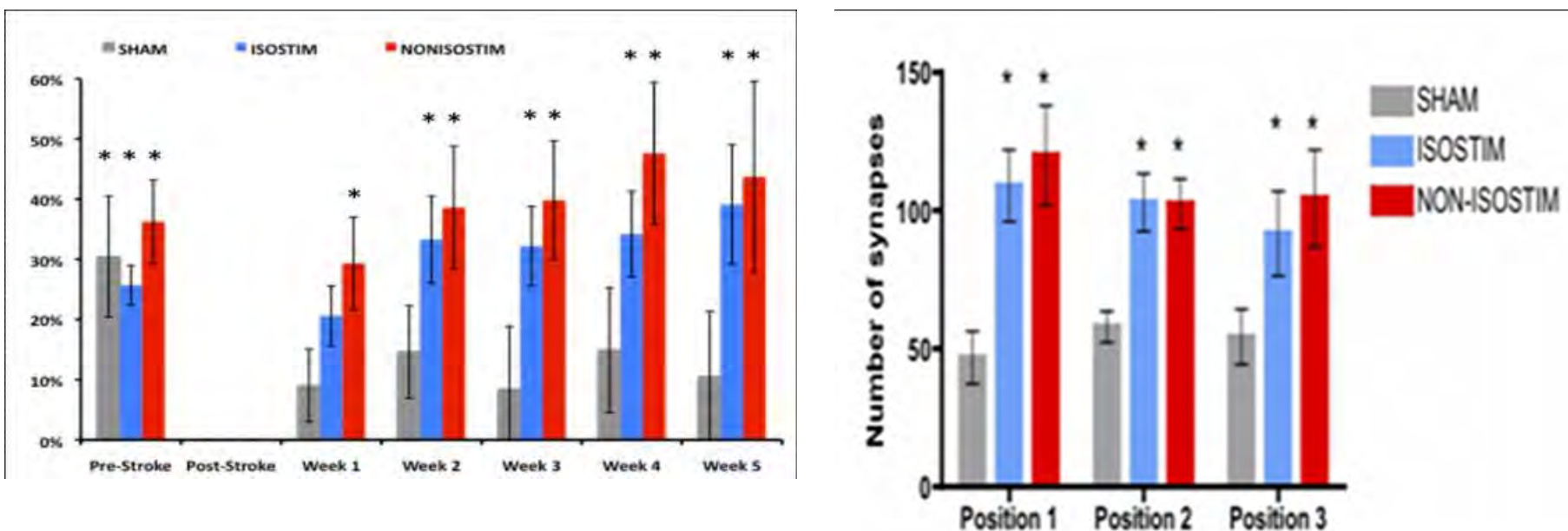


\section{Presentation Number: 29}

\section{Spinal Cord Stimulation For The Treatment Of Minimally Conscious State Patients}

Takamitsu Yamamoto, MD, PhD, Nihon Univ. School of Medicine, Tokyo, Japan; Kentaro Shimoda, MD, PhD, Tokyo, Japan; Mitsuru Watanabe, MD,PhD, Tokyo, Japan; Kohichiro Sumi, MD.PhD, Tokyo, Japan; Toshikazu Kano, MD,PhD, Tokyo, Japan; Toshiki Obuchi, MD,PhD, Tokyo, Japan; Kazutaka Kobayashi, MD,PhD, Tokyo, Japan; Hideki Oshima, MD,PhD, Tokyo, Japan; Chikashi Fukaya, MD,PhD, Tokyo, Japan; Atsuo Yoshino, MD,PhD, Tokyo, Japan; Yoichi Katayama, MD,PhD, Tokyo, Japan

Introduction: The minimally conscious state (MCS) is characterized by inconsistent but clearly discernible behavioral evidence of consciousness and can be distinguished from coma and the vegetative state (VS). MCS patients were evaluated neurologically and electrophysiologically over three months after the onset of brain injury. The long-term results of spinal cord stimulation (SCS) were examined and compared with the those of deep brain stimulation (DBS) for the treatment of the vegetative state (VS).

Methods: Ten MCS patients were treated by SCS. A flexible four-contact, cylindrical electrode was inserted into the epidural space of the cervical vertebrae, and placed at the cervical levels C2 - C4. Five-hertz cervical stimulation was applied for 5 min every 30 min during the daytime at an intensity that produced motor twitches of the upper extremities.

Results: Among the 10 MCS patients, eight satisfied the electrophysiological inclusion criteria (Eur J Neurosci 32: 1145-1151, 2010), that we proposed on the basis of the results of DBS for the treatment of VS patients. Seven MCS patients recovered from MCS following SCS therapy and were able to carry out functional interactive communication and/or demonstrate the functional use of two different objects. C2 - C4 SCS increased cerebral blood flow (CBF) diffusely in the brain, and CBF increased by $22.2 \%$ during the stimulation period compared with CBF before stimulation in MCS patients $(p<0.0001$, paired t-test).

Conclusions: Considering the persistence of physical limitations after the recovery from VS treated by DBS, as we previously reported, we consider that MCS is a good candidate for cerebrospinal stimulation therapy. Five-hertz cervical-level SCS could increase CBF and induce muscle twitches of the upper extremities. Such a stimulation method may be suitable for treating MCS, and MCS may be a good candidate for SCS therapy. 
Presentation Number: 244

\section{Peripheral Nerve Stimulation (pns) And Peripheral Nerve Field Stimulation (pnfs) For The Treatment Of Chronic Neuropathic Pain}

Emil D. Isagulyan, The N.N.Burdenko Institute of Neurosurgery of RAMS, Moscow, Russian Federation; Vladimir Shabalov, Moscow, Russian Federation; Ekaterina M. Salova, Moscow, Russian Federation

Objectives. We aim to present our results of this methods for the treatment of chronic neuropathic pain syndromes.

Material and method. A total number of 41 patients were treated with PNS and PNFES for the last four years. There were 17 males and 24 females, aged 29 - 72 years (mean 40,1 years).Five patients suffered from postthoracotomic pain syndrome, 12 patients - post herpetic neuralgia. 20 patients had pain syndromes secondary to neuropathy of various peripheral nerves. The last four patients with failed back surgery syndrome (FBSS) had pain in the low back area, that was not treatable with spinal cord stimulation (SCS).

Mean follow-up was about 3 years.

Results. The immediate postoperative results were: complete pain relief was achieved in 8 patients $(19,5 \%)$; in 21 patients $(51,2 \%)$ pain relief was ranged 50 to $75 \%$ in comparison with baseline; in 12 patients $(12,5 \%)$ pain improvement was less than $50 \%$. At followup (to 36 month) 6 from 8 patients keep beneficial pain control with neurostimulation; 25 patients $(60,9 \%)$ keep 50 to $75 \%$ pain relief in comparison with baseline (persistent good outcome); 12 patients had satisfactory pain relief; finally in 4 cases initial benefit of stimulation was gradually lost. Overall, 37 patients significantly decrease doses of analgesics (mean by $65 \%$ ) due to neurostimulation, and in 9 cases patients stopped to use analgesics. Daily life activity increase (mean by $70 \%$ ) was noted.

Discussion. PNS and PNFES are an effective techniques for the treatment of severe neuropathic pain. Adherence to exact patient selection criteria is essential for improving of long-term effectiveness of neurostimulation. The further researches are necessary (based on the evidential principles) for conclusion about criteria, which could influence long-term efficiency of this technics. 
Session Title: Papers Session - Psychiatric Surgery

Session Time: Thursday, May 30, 2013, 2:00 pm - 3:00 pm

Presentation Number: 293

Neurosurgery for Psychiatric Disorders in the People's Republic of China- Responsibilities of International Societies Hemmings C. Wu, K.U.Leuven, Leuven, Belgium; Loes Gabriëls, Leuven, Belgium; Bart Nuttin, Leuven, Belgium

Introduction: The Ministry of Health of the People's Republic of China issued in April 2008 the regulation "Notification Regarding Improvement of Management and Related Issues in Neurosurgery for Psychiatric Disorders from General Office of Ministry of Health", in order to ensure medical health care quality and to protect patients' safety. This notification consists of three major regulations: specific requirements for hospitals to perform neurosurgery for psychiatric disorders, rigorously defined indication for neurosurgery for psychiatric disorders, and improved management of clinical research in neurosurgery for psychiatric disorders. The development of this notification, based on consensus of international societies, is undoubtedly a step forward in neurosurgery for psychiatric disorders in China. However, it was only published in Chinese to our knowledge, and therefore remained incomprehensible to non-Chinese-speaking communities.

Methods: Today an English version of the Notification, translated by one of the authors, conveys the perspective of the Chinese government on neurosurgery for psychiatric disorders globally. Moreover, a literature research, including journal articles published in both Chinese and English languages, is conducted to review modern-day neurosurgery for psychiatric disorders in China.

Results: The number of published Chinese articles about clinical studies of neurosurgery for psychiatric disorders decreases significantly since the release of the notification, e.g. the number of published Chinese articles of neurosurgery for schizophrenia decreases from 33 in 2007 to 3 in 2012.

Conclusion: Currently the Committee for Neurosurgery for Psychiatric Disorders, as part of the WSSFN, and the ESSFN, partnering with the Working Group 'Deep Brain Stimulation in Psychiatry-Guidance for Responsible Research and Application', are working together with many other international societies, in hopes of building a set of guidelines to serve on a global scale. Guidelines as such, endorsed by world societies involved in neurosurgery for psychiatric disorders, may guide the future of this field in the right direction worldwide. 
Session Title: Papers Session - Psychiatric Surgery

Session Time: Thursday, May 30, 2013, 2:00 pm - 3:00 pm

Presentation Number: 160

\section{Long-term Follow-up Of Deep Brain Stimulation For Refractory Obsessive-compulsive Disorder}

Young Cheol Na, MD, Yonsei University College of Medicine, Seoul, Korea, Republic of; Ji Hee Kim, MD, Seoul, Korea, Republic of; Won Hee Lee, MD, Seoul, Korea, Republic of; Sang Keum Park, Seoul, Korea, Republic of; Won Seok Chang, MD, Seoul, Korea, Republic of; Hyun Ho Jung, MD, Seoul, Korea, Republic of; Jin Woo Chang, MD,PhD, Seoul, Korea, Republic of

Introduction: Our objective was to investigate the long-term effects of deep brain stimulation (DBS) targeting the ventral anterior limb of the internal capsule and adjacent ventral striatum (VC/VS) using conventional electrodes and stimulation techniques for the treatment of refractory Obsessive-compulsive disorder (OCD).

Methods: Four patients diagnosed with OCD underwent bilateral VC/VS DBS. An average of 3 weeks after implantation, DBS was turned on and a survey was conducted to identify negative and positive effects of acute electrode stimulation. DBS frequency ranged between 90 and $130 \mathrm{~Hz}$, with a pulse width range of 90-270 $\mu$ s, and an amplitude range of 2-5 V. All patients underwent psychiatric examination at pre-op baseline. After implantation, psychiatrists assessed all patients with the Yale-Brown Obsessive Compulsive Scale (Y-BOCS), the Hamilton Rating Scale for Depression (HAM-D) and Global Assessment of Functioning (GAF) scale every 3 months for 24 months.

Results: When Y-BOCS scores were categorized based on percentage improvement, all four patients met the criteria for full response (Y-BOCS change of at least 35\% from baseline) with the improvement rate of $59.7 \pm 14.6 \%$ after 24 months of DBS. At 24 months, HAM-D scores were $7.0 \pm 1.4$, representing a decrease of at least $50 \%$. At preoperative baseline evaluation, all four patients scored less than 45 on the GAF scale. Individual GAF scores from patient 1 to patient 4 were 70, 60, 62, and 60 at 24 months after DBS. There were no serious adverse events related to the surgery.

Conclusions: There were prolonged improvement of obsessive-compulsive symptoms and depressive symptoms along with notable functional improvement in all participants with no battery depletion. 
Session Title: Papers Session - Psychiatric Surgery

Session Time: Thursday, May 30, 2013, 2:00 pm - 3:00 pm

Presentation Number: 26

\section{Deep Brain Stimulation For Severe Treatment-resistant Depression}

RODRIGO RODRIGUEZ, MD, Hospital de la Santa Creu i Sant Pau, Barcelona, Spain; JOAN MOLET, MD, PhD, Barcelona, Spain; PATRICIA PUERTA, MD, Barcelona, Spain; VICTOR PEREZ, MD,PhD, Barcelona, Spain; DOLORS PUIGDEMONT, MD, Barcelona, Spain; ALEXANDRE GIRONELL, MD, Barcelona, Spain

Introduction: Between $30-40 \%$ of patients with major depression do not improve in a first therapeutic trial and between 5 and $10 \%$ did not improve despite the use of more aggressive treatment.

There are evidence of functional hyperactivity in cingulate subcallosal $\mathrm{Cg} 25$ area and hypoactivity of frontal lobes in depressive patients. This pattern is reversed when there is an effective response to treatment.

Methods: We designed a prospective, double-blind, randomized and controlled crossover clinical trial by deep brain stimulation (DBS) of $\mathrm{Cg} 25$ area in patients with severe treatment-resistant depression.

Eight patients suffering from major depression according to DSM IV-TR were operated on. The inclusion criteria were a Hamilton

Depression Rating Scale (HDRS) greater or equal at 18, older than 18, treatment resistant depression and not sustained improvement using electroconvulsive therapy (ECT). All patients were randomized to be the generator switch on or switch off at 3 months and crossover at 6 months in a double-blind condition.

The electrodes were placed in the $\mathrm{Cg} 25$ area bilateral. The stimulation was bipolar at 4,5V, pulse width $150-180 \mu$ s and $135 \mathrm{~Hz}$ of frequency.

Results: The mean HDRS score down from 22 in preoperative condition to 9 at one week after surgery. The HDRS score was 11 at 3 months and 8 at 6 and 12 months. The patients with the stimulation on had a better score than those with the stimulation off. There were not complications related to surgery. Even so, one year later one patient had recurrence of depressive symptoms consummating a suicide attempt.

Conclusions: The $75 \%$ of patients improved 6 months after DBS and in $50 \%$ the depression was in remission. The remission of depression one year after DBS was $70 \%$.

No patients had adverse effects related to DBS. 
Session Title: Papers Session - Psychiatric Surgery

Session Time: Thursday, May 30, 2013, 2:00 pm - 3:00 pm

Presentation Number: 286

\section{Dbs In Tourette Syndrome: Lessons Learnt After 8 Years}

Domenico Servello, IRCCS Galeazzi, Milan, Italy; Edvin Zekaj, Milan, Italy; Mauro Porta, Milan, Italy; Marco Sassi, Milan, Italy; Claudia Menghetti, Milan, Italy

Introduction: Tourette Syndrome (TS) is a neuropsichiatric syndrome caracterized by multiple motor and vocal tics. Psichiatric comorbidities (coexisting) are present in $90 \%$ of patients. First line treatment are pharmacological and psycotherapy. In patient with severe and refractory TS DBS might be considered as a last treatment resort.

Methods: From 2004 to 2012 we have performed DBS in 48 patients. Target chosen were four; Vo-CM/Pf, NA-ALIC, Gpi posteroventral, Gpi antero-medial. All patient underwent psicological evaluation using different scales such as ; YGTSS, Y-BOCS, STAI, BDI.

Results: In 18 patient with long term follow-up (5-6 year), there was a significant reduction in tic severity ( $<<0.001)$, and significant improvements in obsessive compulsive behaviours.

Conclusions: The main topics of DBS in TS are; carefull patient selection, tailored surgical targets, surgical trics for reducing higher device and inflammatory complications. 
Session Title: Papers Session - Psychiatric Surgery

Session Time: Thursday, May 30, 2013, 2:00 pm - 3:00 pm

Presentation Number: 325

\section{The Vancouver Experience with Neurosurgery for Depression: Lesions versus DBS}

Chris Honey, MD, D.Phil., University of British Columbia, Vancouver, BC, Canada; Trevor Hurwitz, MD, Vancouver, BC, Canada;

Ray Lam, MD, Vancouver, BC, Canada; Cindy Woo, MSc, Vancouver, BC, Canada; Alex McGirr, BSc, Vancouver, BC, Canada;

Andrew Howard, MD, Vancouver, BC, Canada

Introduction: There has been a renaissance in the neurosurgery for depression in the last few years led by several studies highlighting the potential use of DBS for mood disorders. The concept of using neuromodulation instead of brain lesioning is appealing to both the referring psychiatrists and their patients. Our centre has experience with both bilateral anterior capsulotomy (BAC) and subcallosal gyrus deep brain stimulation (DBS) for the treatment of medically refractory depression. We present our experience with both of these techniques to highlight the similarities and differences of these two approaches.

Methods: Our University and Hospital institutional review boards approved both i) a long-term study of medically refractory depression patients undergoing bilateral anterior capsulotomy $(\mathrm{n}=8)$ and ii) a pilot study $(\mathrm{n}=5)$ to assess the effects of subgenual cingulate gyrus deep brain stimulation for medically refractory depression. The initial demographic data and inclusion criteria were recorded and then the detailed neuropsychological data were prospectively collected.

Results: A descriptive analysis of the two cohorts will be presented. The outcome at one year post-operative will be presented. The outcome measures for each cohort will be described.

Conclusions: These two neurosurgical procedures for depression ( lesioning and DBS) have many similarities but also have important differences. Although the authors highlight the statistical difficulties with a direct comparison of these two techniques, one conclusion is clear - both procedures are effective in the treatment of medically refractory depression. 


\section{Presentation Number: 8}

Exploring The Anatomical Connectivity Of The Human Pedunculopontine Nucleus With Structural And Diffusion-tensor Mri Yasir Chowdhury, MBBS MSc, UCL Institute of Neurology, Queen Square, London, United Kingdom; Laura Mancini, PhD, London, United Kingdom; John Thornton, PhD, London, United Kingdom; Ludvic Zrinzo, MD PhD, London, United Kingdom

Introduction: Deep brain stimulation of the pedunculopontine nucleus (PPN) has been proposed as a therapy for parkinsonian patients with dopamine refractory gait disturbance. PPN connectivity in humans has mainly been extrapolated from animal studies. Invivo fibre-tracking studies of the PPN in humans are limited by inaccuracies of seed-voxel selection. This study utilises a previously verified method of PPN localisation to analyse the connectivity of subsections of the PPN.

Methods: Diffusion data was acquired from 11 healthy adults using a single shot EPI sequence on a 3T MRI (TR 6800ms, TE $91 \mathrm{ms,}$ 96x96 matrix, 65 directions, $\mathrm{b}=1000 \mathrm{~s} / \mathrm{mm} 2,2.5 \times 2.5 \times 2.5 \mathrm{~mm} 3$ voxels). Optimised structural imaging (TR $18 \mathrm{~ms}$, TE $5.3 \mathrm{~ms}, 176 \times 256$ mm matrix) was used to identify the PPN. Fractional anisotropy images were used to cross reference the structural images to identify the seed voxels that could be confidently concluded to be completely within the PPN. This led to 4 or 5 voxels being selected per PPN with one voxel per axial slice. Probabilistic tractography assessed the strength of connectivity between different regions within the PPN across all subjects.

Results: 22 PPNs were localised by 2 independent raters; out of 92 voxels selected only 3 voxels differed between the 2 raters indicating a high degree of reproducibility in voxel selection ( 2 sample unpaired t-test: $P>0.05$ ). Significant connectivity was evident between the PPN and the thalamus, STN and the globus pallidus. Connectivity between pallidum and inferior PPN voxels was stronger than between pallidum and superior PPN (Z-test: P $<0.05$ ).

Conclusions: This study used a reproducible method of directly visualising the PPN to determine seed voxel selection for connectivity/tractography studies. Known connections between the PPN and basal ganglia were consistently found using this method. Evidence of differential connectivity between superior and inferior PPN are in line with animal studies. 


\section{Presentation Number: 373}

\section{White Matter Tracts Deep To Cg25: What Are We Targeting?}

Kartik D. Bhatia, MBBS, MS, University of Sydney, Darlinghurst, NSW, Australia; Luke Henderson, Darlinghurst, NSW, Australia

Introduction: Brodmann's area 25 (subgenual cingulum: Cg25) is a DBS target for depression that has shown promising early results, and was chosen because of its role as a critical hub in depression pathophysiology. Lead electrodes are inserted at the grey-white matter junction of $\mathrm{Cg} 25$ in order to stimulate the white matter connections between $\mathrm{Cg} 25$ and a variety of structures in the prefrontal limbic-striatopallidal-thalamo-cortical (LSPTC) network. Whilst the connectivity to other structures has been investigated, the actual tracts being stimulated have not been elucidated.

Methods: 75 healthy adult human control subjects were imaged with a 3T scanner for acquisition of T1, T2 and DTI sequences (32directional). For each subject the grey-white matter interface of Cg25 was located bilaterally. Beginning at the target site, DTI was used to assess the tracts traversing each voxel using TrackViz (MGH, 2011). Each tract was separated out using secondary ROIs on their connecting structures with 'and' operations. Tracts were analyzed for connectivity as well as height and width in the coronal plane. This process was repeated at $2 \mathrm{~mm}$ intervals laterally from the grey-white junction of $\mathrm{Cg} 25$ to the Sylvian fissure. Variability of tract location was also measured by standard deviation.

Results: At the grey-white interface of $\mathrm{Cg} 25$, the following tracts were consistently demonstrated: 1) Cingulum bundle, 2) Arcuate fibers to the frontopolar cortex and gyrus rectus, and 3) Hypothalamic connections. Further laterally (in medial-lateral order) was located the amydalo-fugal pathway, ventral striatal connections, uncinate fasciculus (medial stem), stria terminalis, inferior occipitofrontal fasciculus, and the uncinate fasciculus (lateral stem).

Conclusions: The white matter tracts deep to $\mathrm{Cg} 25$ can be individually imaged and potentially targeted. There is wide variability in the exact inter-individual location of each tract lateral to the grey-white interface, suggesting DTI may aid in direct targeting of affected white mater

Tracts.

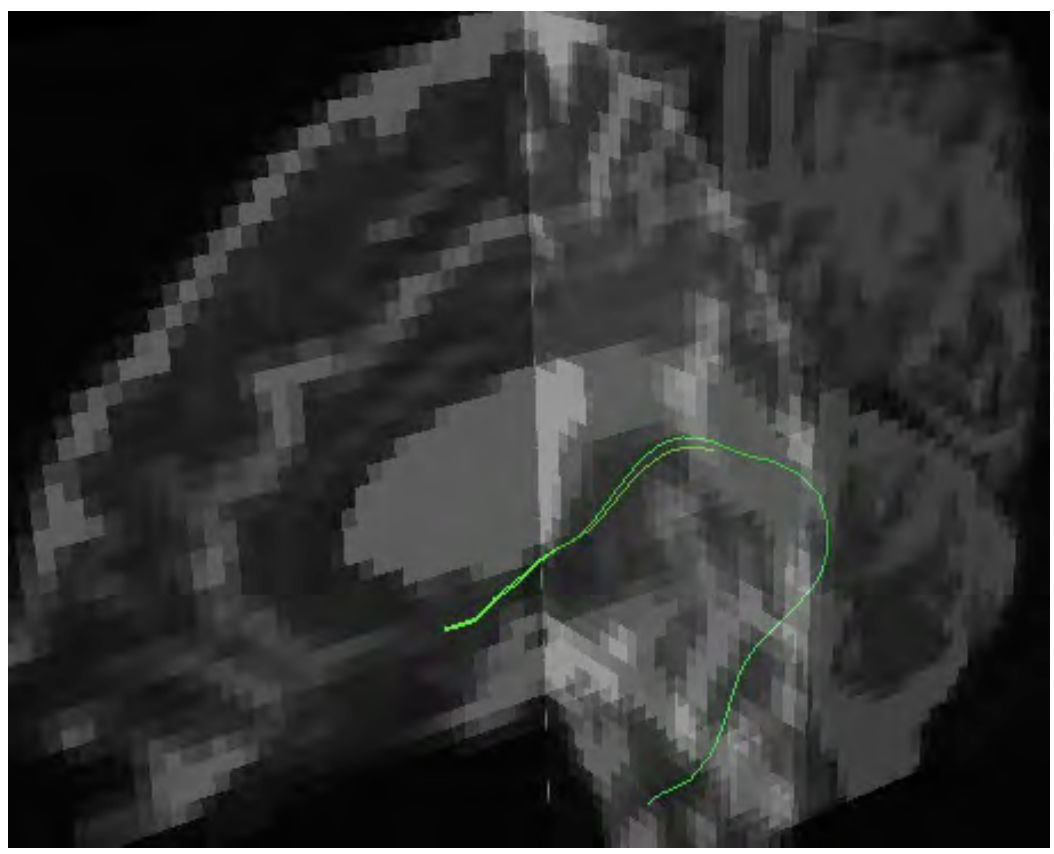


Presentation Number: 267

\section{Early Experience of Pre- and Post-contrast 7.0T MRI in Brain Tumors}

Jae Ha Hwang, Seoul National University Hospital, Seoul, Korea, Republic of; Seung Leal Paek, Rochester, MN; Emily Knight, Rochester, MN; Sun Ha Paek, Seoul, Korea, Republic of; Chul-Ho Sohn, Seoul, Korea, Republic of; Young Don Son, Incheon, Korea, Republic of; Young Bo Kim, Incheon, Korea, Republic of; Dong Gyu Kim, Seoul, Korea, Republic of; Kendall H. Lee, Rochester, MN; Zang-Hee Cho, Incheon, Korea, Republic of

Introduction: We investigated the safety and clinical applicability of pre- and post-contrast 7.0Tesla (T) brain magnetic resonance images (MRIs) in patients with brain tumors.

Methods: Twenty-four patients with intraaxial or extraaxial brain tumors of World Health Organization (WHO) grade I to IV were enrolled in this study. 7.0T MRIs of T2*-weighted axial and T1-weighted coronal or sagittal images with/without contrast medium were obtained and compared with 1.5T brain MRIs. Pre- and post-contrast 7.0T brain MRIs were safely carried out in all twenty-four patients with brain tumors.

Results: The T2*-weighted images from the 7T brain MRI revealed detailed microvasculature and the internal contents of supratentorial brain tumors better than that of the $1.5 \mathrm{~T}$ brain MRI. Pre- and post-contrast T1 weighted images from the 7.0T brain MRI with contrast enhancement provided sharpened images and detailed information on the contents and their surrounding neurovascular structures of the supratentorial brain tumors. For brain tumors located in parasellar areas or areas adjacent to major cerebral vessels, flow-related artifacts were exaggerated in the 7.0T brain MRIs. For brain tumors adjacent to skull base, susceptibility artifacts in the interfacing areas of paranasal sinus and skull base hampered the aquisition of detailed images and information on brain tumors in the 7.0T brain MRIs.

Conclusions: This study demonstrated that pre- and post-contrast 7.0T brain MRI can be safely performed in patients with brain tumors. Furthermore, it can provide detailed information on the intratumoral components and margins in supratentorial brain tumors. However, further study is needed to develop refined MRI protocol for the better images of brain tumors located in skull base, parasellar, and adjacent major cerebrovascular structures. 


\section{Presentation Number: 224}

\section{Localization of DBS Targets by Deterministic Tractography: A Straightforward Method with Plausible Results} Josue Avecillas, MD, Hospital Clinico San carlos, Madrid, Spain; Juan Barcia, MD, PhD, Madrid, Spain; Juan Alvarez-Linera, Madrid, Spain

Introduction: The understanding of functional circuits in the brain has been of critical importance in DBS. Despite advances in Neuroimaging, the mapping of these circuits remains elusive. DTI has been widely used in the clinical setting to identify the main white matter tracks with several limitations. Identification of specific targets in DBS is very difficult to achieve, even in the most powerful MRI systems. We present a method based on DTI and performed in navigation software, which could lead to identify the DBS targets.

Methods: We used T1, T2, FLAIR and DTI 3T MRI scans of six patients operated for DBS in this work. Using StealthViz Software, through the FACT algorithm, we segmented the targets and projection areas based in anatomical landmarks. The projection areas were cortical regions and the targets were within the basal ganglia. With a subtraction method, we were able to isolate regions in the targets coincident with functional subdivisions of the basal ganglia, described in the literature. These results were ascertained, comparing them with classical coordinates of the targets of DBS

Results: We were able to identify regions coincident with the motor and limbic parts of STN, Nucleus Accumbens, Ventral Intermediate Nucleus, DRT tract and Dorsomedial thalamic nucleus. We performed a reconstruction of the Motor, Associative, Limbic and Hyperdirect circuits of the basal ganglia, using this method, through anatomical landmarks

Conclusions: Despite the limitations of Deterministic Tractography (DT), we obtained plausible results. This method is straightforward and is performed in navigation software, with the possibility to utilize these regions during surgery as additional landmarks or explore postsurgically the results of stimulation. Further research is needed, correlating the regions with the clinical results in order to validate this type of methods.
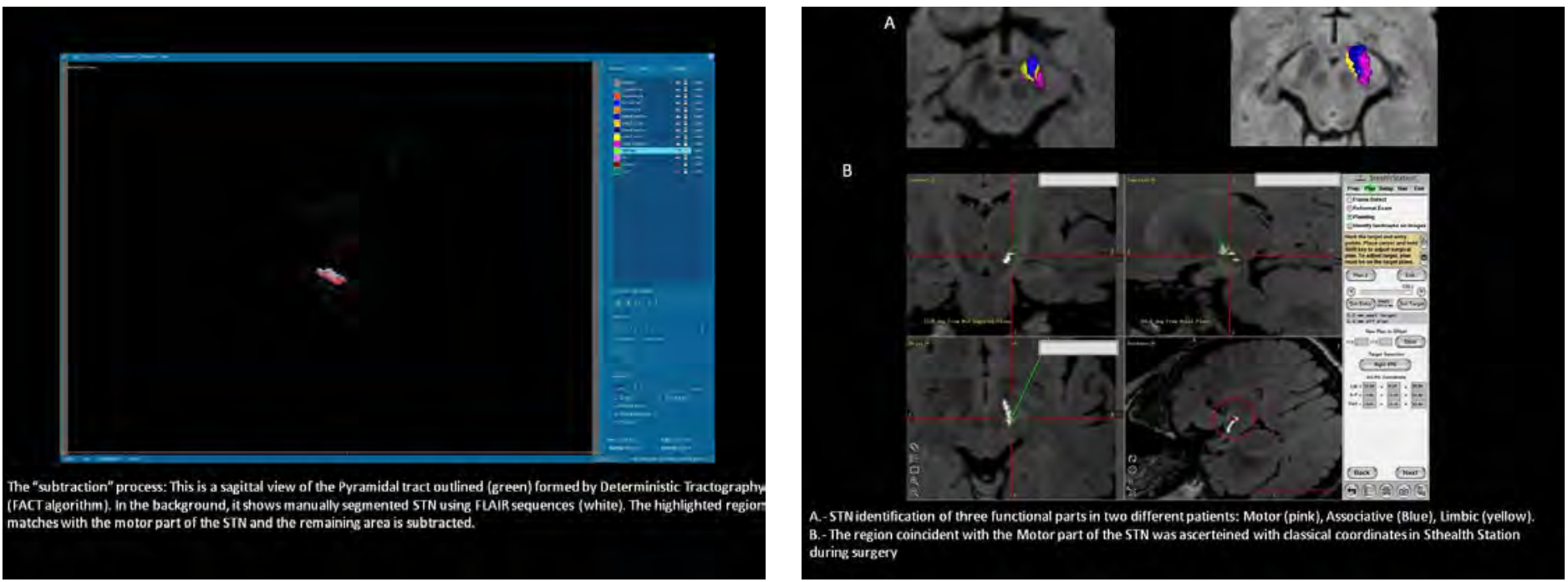
Deep Brain Stimulation Of The Dentato-rubro-thalamic Tract (drt) For The Treatment Of Therapy Refractory Tremor Volker A. Coenen, M.D., Stereotactic and Functional Neurosurgery, Freiburg University Neurocenter, Freiburg, Germany; Bastian Sajonz, MD, MSc, Stereotaxy and Functional Neurosurgery, Bonn Univ., Germany; Sebastian Paus, MD, Department of Neurology, Bonn University, Germany; Niels Allert, MD, Neurological Rehab Facility "Godeshöhe", Bonn, Germany; Horst Urbach, MD, Department of Radiology/Neuroradiology, Bonn Univ., Germany; Burkhard Maedler, PhD, Stereotactic and Functional Neurosurgery, Freiburg, Germany

Introduction: DBS alleviates tremor of various origin. A number of regions like the ventralis intermediate nucleus of the thalamus (Vim), the caudal zona incerta (cZI) and the posterior subthalamic area (pSTN) are targeted. In recent work the involvement of the dentato-rubro-thalamic tract (drt) in tremor control was reported with fiber tracking techniques. We report the results of a prospective trial that scrutinizes the role of the drt.

Methods: A total of $n=11$ patients were enrolled. There were 6 male patients (64+/-17 years). Diagnoses were: Essential Tremor (6), myoclonus dystonia (2), Parkinson's disease (3). Patients were surgical candidates according to established guidelines. They were enrolled after written informed consent. The evaluation of the DTI data and their publication were approved by the institutional ethics board (no. 199/09). Patients received one (3), two (7), or three (1) quadripolar DBS electrodes (model 3389 Medtronic, USA). Electrodes were implanted stereotactically under local anesthesia and with intraoperative testing. A 32-direction DTI sequence was acquired preoperatively, together with anatomical and structural imaging. DTI was not used for planning and only processed postoperatively. Vim DBS was performed with standard techniques. The DRT was tracked and visualized individually. Postoperative helical CT was used to determine electrode positions and fused to preoperative MRI data. Individual tracking results were warped to the ICBM152 normalized brain space together with individual effective electrode contact positions. Electric fields (EF) were simulated and visualized according to the stimulation parameters.

Results: In all patients tremor was alleviated effectively. If tremor-reduction was moderate (2) the EF was located at the anterior border of the drt only partially covering it. In good tremor reduction (9) the EF enclosed the drt.

Conclusions: Neuromodulation of the drt is likely to produce tremor-reducing effects. The drt connects the traditional target regions for DBS in tremor disease.

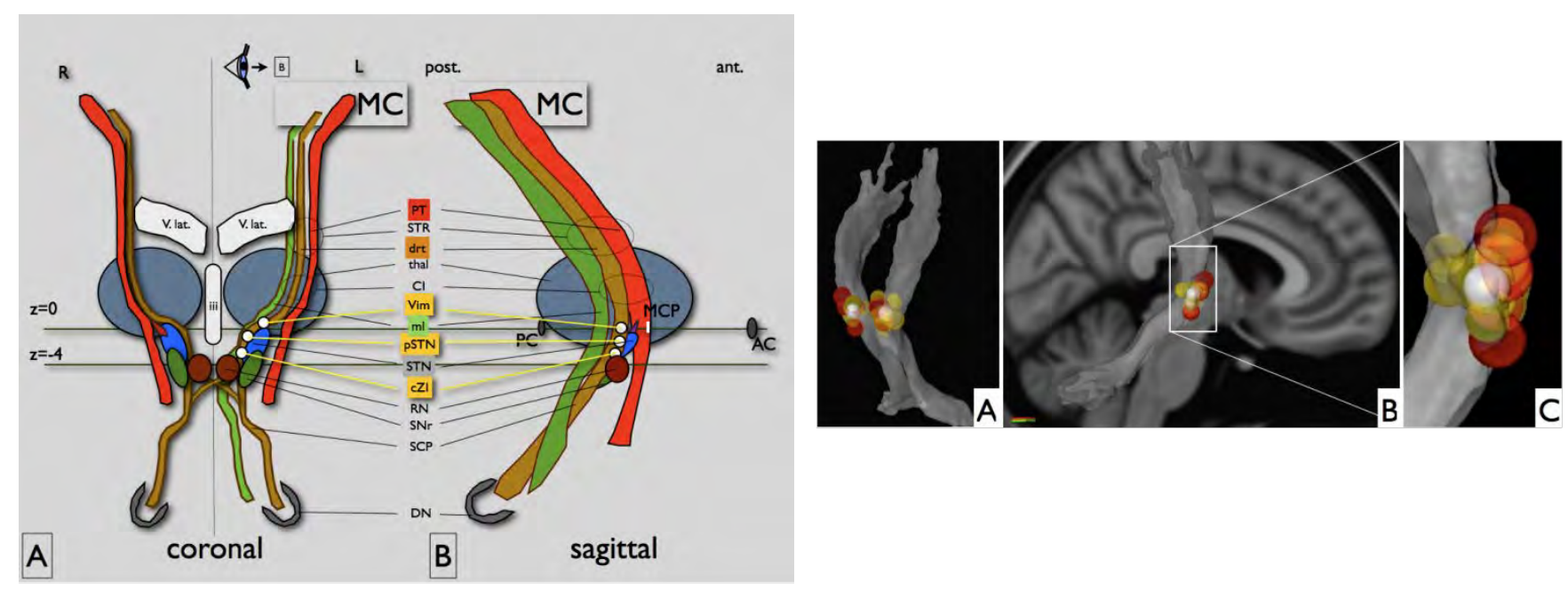




\section{Presentation Number: 87}

\section{Zona Incerta As A Target For DBS - The Umeå Experience Of 100 Patients}

Patric Blomstedt, MD, PhD, Department of Neurosurgey, University Hospital of Umeå, Umeå, Sweden

Introduction: The caudal Zona incerta (cZi) has been suggested as a target for DBS in movement disorders, especially as an alternative to Vim for tremor, but also to the Subthalamic nucleus (STN) in non-tremor dominated Parkinson's disease (PD). The results from 10 patients with non-essential tremor (ET)/non-PD, 18 patients with ET, and 42 patients with PD have been presented in 10 studies from other groups. Based on our first 100 patients with Zi-DBS, we present our experience concerning this target regarding indications, results, programing, intra-operative evaluation, targeting, and the possibility to perform these procedures in general anesthesia based on visual targeting.

Methods: Nine patients with non-ET/PD, 53 with ET and 38 with PD were implanted in the cZi and analyzed in different sub-studies. Results: cZi DBS provided excellent results in dystonic, writer's and cerebellar tremor, but modest or poor results in neuropathic, orthostatic, post-stroke and post-traumatic tremor. In ET an improvement of $89 \%$ was achieved concerning hand tremor and function. The effect was stable over time ( 5 years) and no tolerance occurred. Parkinsonian tremor was reduced with $82 \%-90 \%$. The improvement on rigidity and hypokinesia in patients eligible for STN DBS was more limited (39\%). No ICB or other serious complication occurred in this group. The target can be identified visually by its relation to the STN and the red nucleus. The area has characteristic properties, which must be considered when optimizing the settings for chronic stimulation. Intra-operative stimulation is, however, of very limited value, if the electrode is correctly placed according to the visual anatomy. The procedure is therefore currently being performed in all patients, including ET, in general anesthesia.

Conclusion: In our experience the $\mathrm{cZi}$ is safe and efficient target providing the possibility to perform DBS for tremor in general anesthesia.

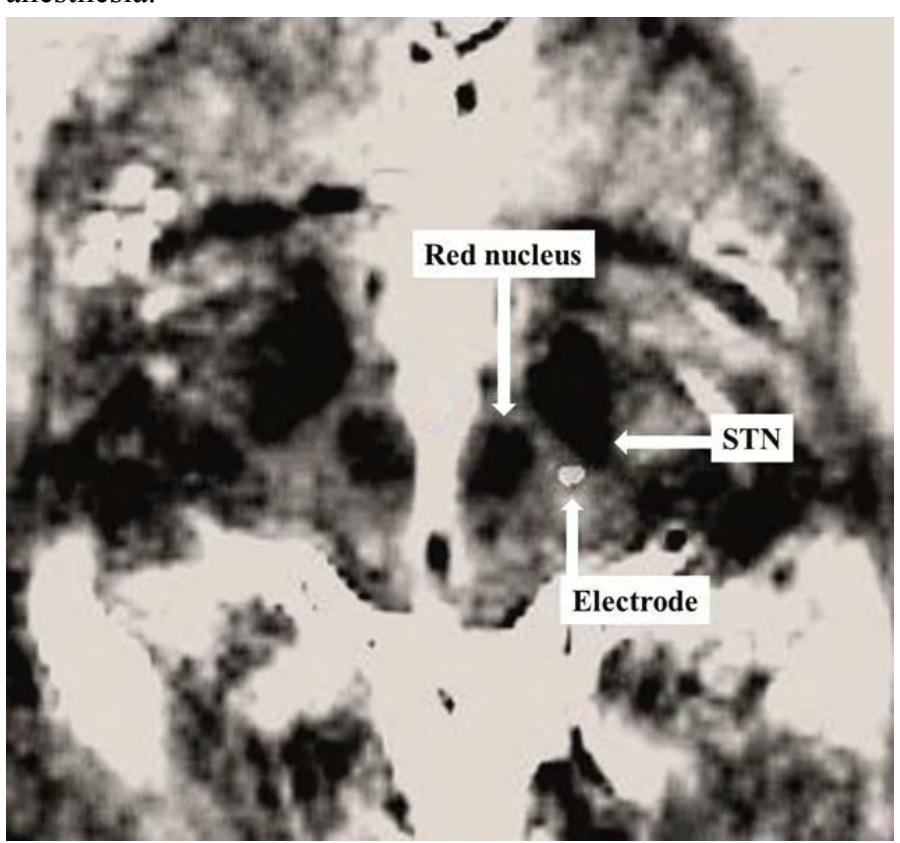




\author{
Anatomo-clinical Correlations Of Deep Brain Stimulation (DBS) Of The Pedunculopontine Nucleus Area (PPNa) At 12 \\ Months Follow Up \\ Laurent GOETZ, INSERM U836, Grenoble, France; Valérie FRAIX, MD, Grenoble, France; Manik BHATTACHARJEE, PhD, \\ Grenoble, France; Murielle FERRAYE, PhD, Nijmegen, Netherlands; Carina MAINERI, MD, Buenos Aires, Argentina; Brigitte \\ PIALLAT, PhD, Grenoble, France; Olivier DAVID, PhD, Grenoble, France; Albert FENOY, MD, Houston, TX; Napoleon TORRES, \\ $\mathrm{MD}, \mathrm{PhD}$, Grenoble, France; Bettina DEBU, PhD, Grenoble, France; Eric SEIGNEURET, MD, Grenoble, France; Alexandre \\ KRAINIK, MD,PhD, Grenoble, France; Pierre POLLAK, MD,PhD, Genève, Switzerland; Alim-Louis BENABID, MD,PhD, \\ Grenoble, France; Stéphan CHABARDES, MD, Grenoble, France
}

Introduction: Following experimental data, DBS of the Pedunculopontine nucleus (PPN), was proposed as a new therapy to treat gait disorders in Parkinson's disease (PD). Despite a growing interest, several studies pointed variabilities in clinical responses. The position of the active contacts within the Tegmentum could explain such heterogeneity.

Using a new brainstem normalized coordinate system (BNCS) based on ponto-mesencephalic junction (PMJ) landmarks, we aimed at comparing the positions of the active contacts among a series of 11 patients at 12 months follow up. Also, we propose a probabilistic target based on the best clinical outcomes obtained and on intra-operative micro-stimulation mapping.

Methods: Eleven PD patients (8 already received STN implantation), were included in this prospective study.

Clinical evaluations were based on composite gait score. Positions of active contacts and microstimulation sites were calculated in the standard Talairach coordinate system then transformed onto the BNCS in order to calculate the normalized coordinates. All active contacts were plotted onto a PD template MR-image.

Results: Patients were classified as "bad responders" ( 2 patients), "mild responders" ( 1 patient) and "good responders" (6 patients). Two patients were excluded of the analysis due to severe cognitive decline and post-op heavy fall.

Most effective contacts were located in the posterior part of the Tegmentum (containing posterior PPN and cuneiform nucleus) at the level of the PMJ. We defined a target point with normalized coordinates in BNCS (Xbn =2/3 ObnXbn; Ybn = 2/5 ObnYbn ; Zbn = JPM). Intraoperative side effects mapping at $25 \mathrm{~Hz}$ microstimulation (mainly paraesthesia and oscillopsia) allowed to avoid miss positioning of the chronic lead.

Conclusions: We propose a probabilistic, normalized DBS target based on anatomo-clinical correlations obtained at 12 months follow-up. Precice refinement using microstimulation provide informations to define an anatomical window allowing a safe lead implantation in the PPN area. 
Session Title: Papers Session - Movement Disorders II

Session Time: Thursday, May 30, 2013, 2:00 pm - 3:00 pm

\section{Presentation Number: 95}

\section{Beta Oscillatory Activity Of Single Neurons In The Subthalamic Nucleus In Patients With Parkinson's Diseas}

Kazutaka Kobayashi, MD, PhD, Nihon University School of Medicine, Tokyo, Japan; Mitsuru Watanabe, MD, PhD, Tokyo, Japan; Koichiro Sumi, MD, PhD, Tokyo, Japan; Toshiki Obuchi, MD, PhD, Tokyo, Japan; Toshikazu Kano, MD, PhD, Tokyo, Japan; Hideki Oshima, MD, PhD, Tokyo, Japan; Chikashi Fukaya, MD, PhD, Tokyo, Japan; Atsuo Yoshino, MD, PhD, Tokyo, Japan; Takamitsu Yamamoto, MD, PhD, Tokyo, Japan; Yoichi Katayama, MD, PhD, Tokyo, Japan

Introduction: Recording of local field potentials in the subthalamic nucleus (STN) in Parkinson's disease (PD) patients has shown increased beta-band activity, which is related to akinesia. On the other hand, analysis of neural activity of a single neuron in PD patients has shown increased neuronal firing in the STN, which could cause akinesia. However, both beta-band activity and increased neuronal firing are not satisfactory evidences for all symptoms of PD. In this study, we investigated beta-band activity of a single neuron and analyzed its relationship with neuronal firing rate.

Methods: We analyzed the frequency of a total of 107 single neurons in ten patients with advanced PD, who underwent implantation of deep brain stimulation electrodes in the STN, and classified the neurons as beta-band neurons (neurons with significant oscillatory activity at the beta-band) and non-oscillatory neurons (neuron without significant oscillatory activity at any band). The two groups were compared for firing rate, distribution within the STN, and proportion of neurons responding to passive joint movement.

Results: Of the 107 neurons, 14 (13\%) and 75 (75\%) were beta-band neurons and non-oscillatory neurons, respectively. The firing rate of beta-band neurons was significantly higher than that of non-oscillatory neurons (median firing rates, $45 \mathrm{~Hz} v \mathrm{vs} .25 \mathrm{~Hz}, \mathrm{p}<$ 0.05). Beta-band neurons were widely distributed within the STN, and there was no significant intergroup difference in neuron distribution within the STN. We also noted no significant intergroup differences in the proportion of neurons responding to passive joint movement. Conclusions: The higher firing rate of beta-band neurons was consistent with the firing rate model of akinesia. The dorsal part of the STN is known as the motor area, but the beta-band activity recorded from a single neuron may not specifically reflect the motor symptoms of PD because of the wide distribution of beta-band neurons. 


\section{Anatomo-radiologic Correlation To Achieve Maximum Accuracy In Leads Implantation For Chronic Stimulation Of Sphenopalatine Ganglion}

Fabián C. Piedimonte, MD, Fundación CENIT para la Investigación en Neurociencias, Ciudad Autónoma de Buenos Aires, Argentina; Juan Carlos M. Andreani, MD, Ciudad Autónoma de Buenos Aires, Argentina; Leandro R. Piedimonte, MD, Ciudad Autónoma de Buenos Aires, Argentina; Nicolás E. Barbosa, MD, Ciudad Autónoma de Buenos Aires, Argentina; Adriana M. Pérez, MD, Ciudad Autónoma de Buenos Aires, Argentina; Tania Acosta, MS, Ciudad Autónoma de Buenos Aires, Argentina

Introduction: The sphenopalatine ganglion (SPG) is a small parasympathetic structure located in the pterygopalatine fossa close to the sphenopalatine foramen, distal and medial to the maxillary tuberosity. Its fibers supply the oral mucosa of the oropharynx, tonsils, soft and hard palates, and nasal cavity. It has been implicated in diverse facial pain conditions and considered as a potential target by anesthetics blocks, radiofrequency lesions and chronic stimulation. We attempt to provide relevant radiologic landmarks to optimize the location of implanted leads for chronic stimulation.

Methods: We utilized cadaveric human hemi-heads preserved in formalin and performed dissection and exposure of the SPG along with its relevant related anatomical structures. We simulated an infrazygomatic percutaneous approach with a tetrapolar electrode until the direct visualization of the close relation between the electrode's contacts and the SPG. After fixing the electrode in the optimum position and marking the SPG with a metal needle, the specimen was subjected to diverse radioscopic incidences in order to detect the most significant landmarks.

Results: We identified concrete radiologiclandmarks in diverse incidences taking in cadaveric specimens favoring the proximity between the electrode poles and the SPG in order to optimize its stimulation in patients.

Conclusions: We consider that a lead with the distal contact at its end extreme would represent the ideal design in order to obtain the best response to SPG stimulation. Among others, the transnasal, transoral and infrazygomatic approaches have excelled. Only the last one is potentially useful for the consideration of an electrode implant for SPG chronic stimulation, mainly due to the high risk of infective complications related to the first two.
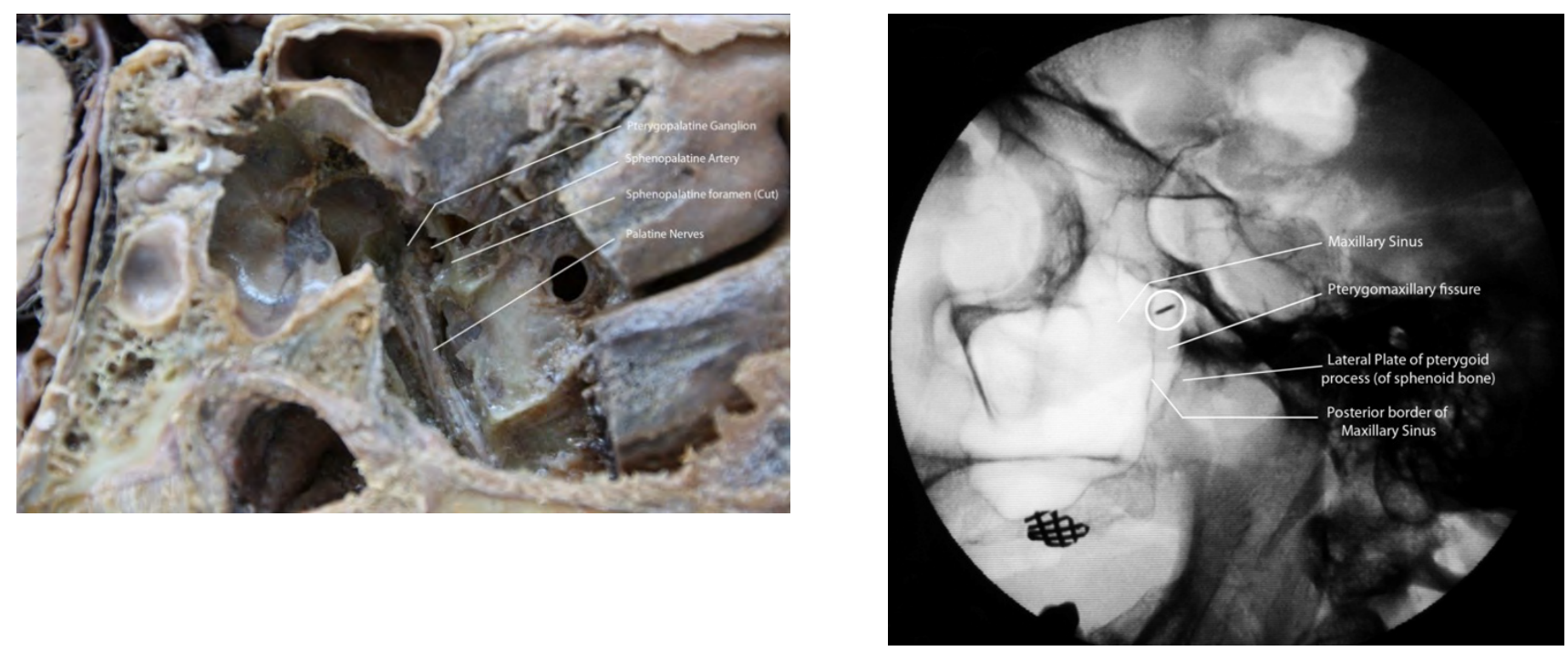


\section{Deep Brain Stimulation Induces Changes In Cerebral Perfusion In Patients With Tourette Syndrome}

Cathleen Schmidt, M.D., Hanover, Germany; Kirsten R. Müller-Vahl, Prof., Hanover, Germany; Christoph Schrader, M.D., Hanover, Germany; Götz Lütjens, Medical School Hanover, Hanover, Germany; Hans H. Capelle, M.D., Hanover, Germany; Frank M. Bengel, Prof., Hanover, Germany; Joachim K. Krauss, Prof., Hanover, Germany; Georg Berding, Prof., Hanover, Germany

Introduction: Nothing is known about changes of regional blood flow under pallidal and thalamic deep brain stimulation in patient with TS. Therefore we evaluated changes of regional cerebral blood flow (rCBF) in patients with severe Tourette syndrome (TS) as a result of bilaterally deep brain stimulation (DBS) of the globus pallidus internus (GPi) and the centromedian-parafascicular/ ventralis oralis internus of the thalamus (CM/ Voi).

Methods: We enrolled 5 severely affected TS patients (age $31 \pm 10$ ys, YGTS-Scale $81 \pm 8$ ) with DBS electrodes in the GPi and $\mathrm{CM} /$ Voi and 6 age-matched, healthy controls for Tc-99m ECD SPECT. Patients were scanned during anaesthesia: 1. before DBS implantation (preOP) and after 3 months of 2. GPi-DBS, 3. CM/Voi-DBS or 4. sham stimulation (OFF), respectively. Data were analysed by voxelwise and volumes-of-interest (VOI) analysis (e.g. frontal lobe and Brodmann areas (BA)) (SPM2 and SPSS statistics, significance thresholds: $\mathrm{p}<0.01$ and 0.05 , respectively).

Results: In patients preOP compared to controls, $\mathrm{rCBF}$ was significantly decreased in the central region, frontal and parietal lobe, as well as in BAs 1, 4-9, 30, 31 and 40. Cerebellar perfusion was increased. DBS significantly decreased rCBF compared to OFF condition: (1.) for GPi-DBS in striatum, pallidum, cerebellum and BA21; (2.) for CM/Voi-DBS in cerebellum and BA19. A significant increase of $\mathrm{rCBF}$ was found during both DBS conditions in the frontal cortex.

Conclusions: We demonstrated reduced frontal cortex perfusion in non-stimulated TS patients particularly in parts involved in planning and controlling of movements. Perfusion changes due to DBS might be correlates of clinically beneficial effects i.e. reduced perfusion in basal ganglia / cerebellum during GPi- or CM/Voi-DBS reflecting reduced presence of motor-tics and increased perfusion during $\mathrm{CM} /$ Voi-DBS in frontal cortex (BA10) reflecting improved motor control 


\section{Localisation Of The Subthalamic Nucleus In Parkinson's Disease with Neural Beta and Gamma Activity of Local Field Potentials}

Daphne Zwartjes, MSc, TU, Enschede, Netherlands; Rick Schuurman, Academic Medical Center Amsterdam, Amsterdam, Netherlands; Rens Verhagen, MSc, Amsterdam, Netherlands; Ciska Heida, PhD, TU, Enschede, Netherlands; Fiorella Contarino, MD, $\mathrm{PhD}$, Amsterdam, Netherlands; Rob de Bie, MD, PhD, Amsterdam, Netherlands; Pepijn van den Munckhof, MD, PhD, Amsterdam, Netherlands; Hubert Martens, PhD, Sapiens, Eindhoven, Netherlands; Peter Veltink, PhD, TU, Enschede, Netherlands; Lo Bour, Amsterdam, Netherlands

Introduction: To refine the MRI-based target during DBS surgery, microelectrode recordings (MER) are often performed to detect target-specific single unit activity. This requires additional recording time and increases the risk for haemorrhage. In the future it may therefore be relevant to be able to determine the implantable lead's position based on local field potential (LFP) recordings from the lead itself which reflect activity of a larger neural population. This study aims to evaluate the nature of oscillatory activity in the subthalamic nucleus (STN) by means of intraoperative LFP-recordings, its relationship with microelectrode recordings and its potential use to locate the STN and its sensorimotor sub-area in patients with Parkinson's disease during deep brain stimulation (DBS) surgery.

Methods: 25 patients with Parkinson's disease are included in this study. MER and LFPs are recorded every $0.5 \mathrm{~mm}$ from multiple microelectrodes during DBS surgery in 48 STNs. A novel optimization approach to map the measurement points on an atlas STN based on the MER properties is used to enable a detailed spatial representation of these points. Power and coherence in different LFP frequencies at all points are assessed in reference to the point's location inside or outside the STN and its sensorimotor sub-area.

Results: Coherence between LFPs and the envelope of spiking activity significantly increases when entering the STN. There is also a pronounced increase in the LFP power in the gamma band, which persists throughout the entire STN in $100 \%$ of the cases. In $70 \%$ of the cases LFPs have a significantly higher power in the high beta frequency band in the sensorimotor STN, defined by the mapping algorithm, compared to the non-sensorimotor STN.

Conclusions: LFP gamma oscillations provide a useful tool for locating the STN intraoperatively and LFP beta oscillations can become useful to discriminate the sensorimotor area within the STN. 


\section{Deep Brain Stimulation For The Early Treatment Of The Minimal Coscious Sate And Vegetative State}

Darko Chudy, MD, PhD, Clinical Hospital Dubrava, Zagreb, Croatia; Vedran Deletis, MD, PhD, Medical School University of Split, Croatia; Maja Rogic, PhD, Medical School University of Split, Croatia; Veronika Paradzik, Department of neurosurgery, Zagreb, Croatia; Gordan Grahovac, Department of neurosurgery, Zagreb, Croatia

Introduction: An effective treatment of minimal conscious state (MCS) and vegetative state (VS), caused by hypoxic encephalopathy (HE) or traumatic brain injury (TBI), has not been yet achieved. DBS of thalamic reticular nuclei has been attempt mainly in TBI patients.

Methods: Thirteen patients were included (four with TBI and nine with HE, four being in MCS and nine in VS). Entry criteria evaluating status of cerebral cortex and thalamocortial reticular formation comprise of: neurological, including Rappaport Coma/Near coma scale, electrophysiological with multimodal evoked potential and 12/24 hours of EEG, and imaging; positron emission tomography and MRI.

The stimulation target: unilateral centromedian-parafascicular nucleus complex, in 2 patients were bilateral (Fig.1). Patients were stimulated daily for 30 min every three hours (intensity, inducing "arousal reaction", frequency $25 \mathrm{~Hz}$, pulse duration $220 \mu \mathrm{s}$ ). Follow up was from 9 to 26 months.

Results: Two MCS patients regained consciousness, waking, speaking fluently, with impressive speech comprehension. One become completely independent while other needed some assistance in everyday life due to memory deficit (Fig.2). Two VS patients improved to MCS with further recovery after 24 months of DBS. Two died from respiratory infection and sepsis. Other seven patients remain unchanged level of consciousness.

Conclusion: If patients fulfills clinical, neurophysiological, and neuroimaging criteria they should undergo DBS at rather early stage. The spontaneous recovery of MCS/VS is very rare, therefore if entry criteria are fulfilled DBS could be option.

Figures legend:

Fig.1 Patient in MCS(left) and 3 months after DBS(right),

Fig.2 position of lead

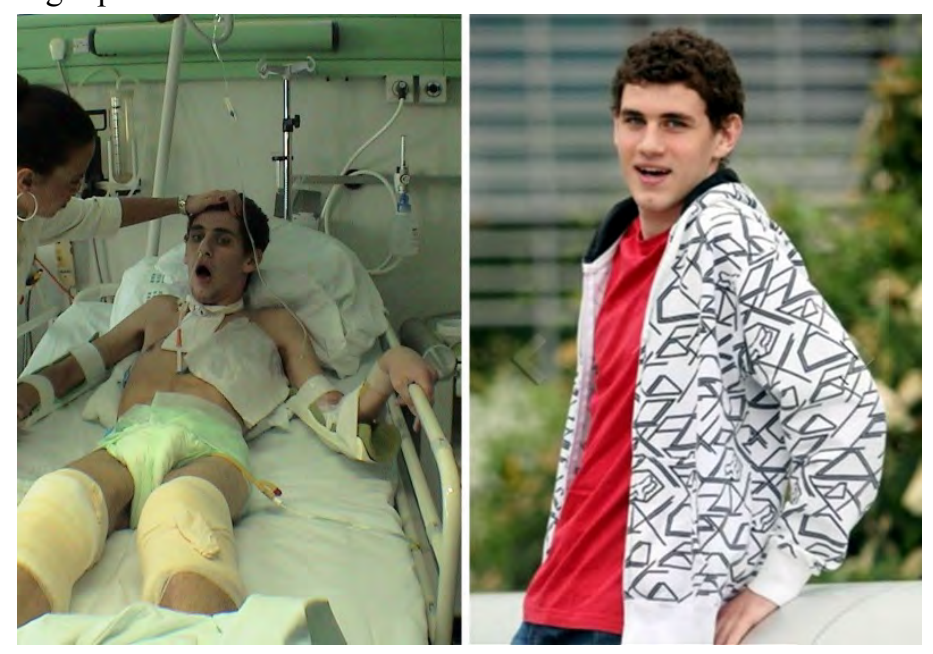

